\title{
WestVirginiaUniversity
}

THE RESEARCH REPOSITORY @ WVU

Graduate Theses, Dissertations, and Problem Reports

2007

\section{Control of a swept wing tailless aircraft through wing morphing}

Richard W. Guiler

West Virginia University

Follow this and additional works at: https://researchrepository.wvu.edu/etd

\section{Recommended Citation}

Guiler, Richard W., "Control of a swept wing tailless aircraft through wing morphing" (2007). Graduate Theses, Dissertations, and Problem Reports. 2779.

https://researchrepository.wvu.edu/etd/2779

This Dissertation is protected by copyright and/or related rights. It has been brought to you by the The Research Repository @ WVU with permission from the rights-holder(s). You are free to use this Dissertation in any way that is permitted by the copyright and related rights legislation that applies to your use. For other uses you must obtain permission from the rights-holder(s) directly, unless additional rights are indicated by a Creative Commons license in the record and/ or on the work itself. This Dissertation has been accepted for inclusion in WVU Graduate Theses, Dissertations, and Problem Reports collection by an authorized administrator of The Research Repository @ WVU.

For more information, please contact researchrepository@mail.wvu.edu. 


\title{
CONTROL OF A SWEPT WING TAILLESS AIRCRAFT THROUGH WING MORPHING
}

\author{
by
}

Richard W. Guiler

\begin{abstract}
Dissertation submitted to the College of Engineering and Mineral Resources at West Virginia University in partial fulfillment of the requirements for the degree of
\end{abstract}

\author{
Doctor of Philosophy \\ in \\ Aerospace Engineering \\ Approved by \\ Wade Huebsch, PhD., Committee Chairperson \\ John Loth, PhD. \\ Gary Morris, PhD. \\ John Kuhlman, PhD. \\ Jens Madsen, PhD.
}

Department of Mechanical and Aerospace Engineering

Morgantown, West Virginia

2007

Keywords: Morphing Aircraft Structures, Tailless Aircraft, Horten, Wing Twist, Blended Wing Body and Aircraft Controls

Copyright 2007 Richard W. Guiler 


\begin{abstract}
CONTROL OF A SWEPT WING TAILLESS AIRCRAFT THROUGH WING MORPHING

by Richard W. Guiler

Inspired by flight in nature, work done by Lippisch, the Hortens, and Northrop offered insight to achieving the efficiency of bird flight with swept-wing tailless aircraft. Tailless designs must incorporate aerodynamic compromises for control, which have inhibited potential advantages. A morphing mechanism, capable of changing the twist of wing and that can also provide pitch, roll and yaw control for a tailless swept wing aircraft is the first step to a series of morphing techniques, which will lead to more fluid, bird-like flight. This research focuses on investigating the design of a morphing wing to improve the flight characteristics of swept wing Horten type tailless aircraft. Free flight demonstrators, wind tunnel flow visualization, wind-tunnel force and moment data along with CFD studies have been used to evaluate the stability, control and efficiency of a morphing swept wing tailless aircraft.
\end{abstract}

A wing morphing mechanism for the control of a swept wing tailless aircraft has been developed. This new control technique was experimentally and numerically compared to an existing elevon equipped tailless aircraft and has shown the potential for significant improvement in efficiency. The feasibility of this mechanism was also validated through flight testing of a flight weight version.

In the process of comparing the Horten type elevon equipped aircraft and the morphing model, formal wind tunnel verification of wingtip induced thrust, found in Horten (Bell Shaped Lift distribution) type swept wing tailless aircraft was documented. A more complete physical understanding of the highly complex flow generated in the control region of the morphing tailless aircraft has been developed.

CFD models indicate the possibility of the presence of a Leading Edge Vortex (LEV) on the control section morphing wing when the tip is twisted between +3.5 degrees and +7 degrees. The presence of this LEV causes a reduction of drag while lift is increased. Similar LEVs have been documented in use by birds and insects. 


\section{Dedication}

The author wishes to thank my family for all the support and patience, which made it possible to continue this exciting research.

\section{Acknowledgments}

The author wishes to express my sincere gratitude to my committee for all the help in order to complete this work. I would also like to thank Dr. Karl Nickel and Gunilde Nickel for their support, advice and encouragement. 
Table of Contents

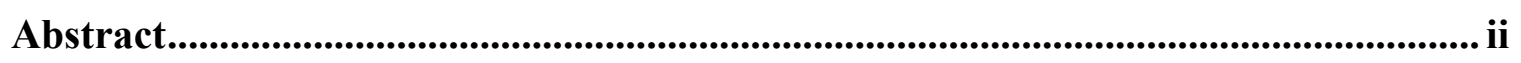

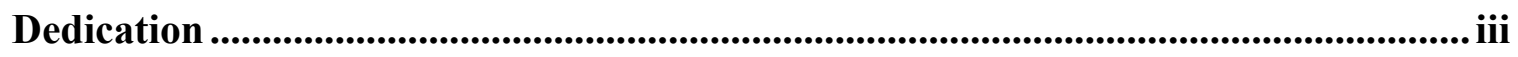

Acknowledgments ......................................................................................................................... iii

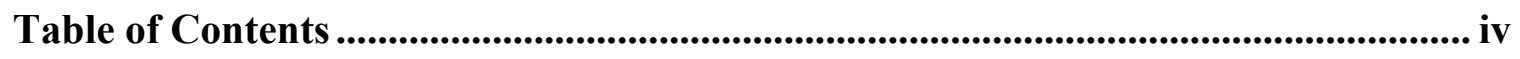

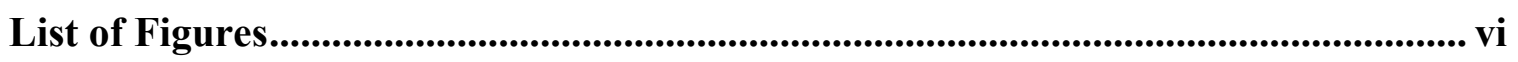

List of Tables ................................................................................................................. xii

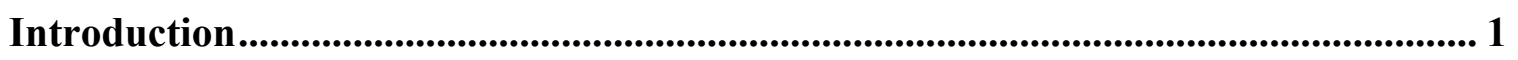

Chapter 1 Swept-wing Tailless Aircraft .............................................................................. 3

1. Early Swept-wing Tailless Aircraft ......................................................... 3

2. Alexander Lippisch .....................................................................................5

3. John K. Northrop....................................................................................... 13

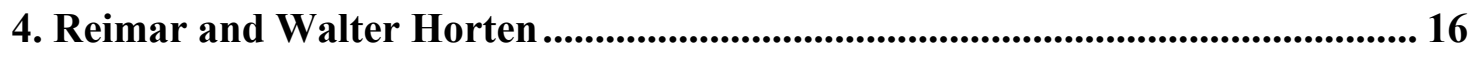

5. Recent Swept Wing Tailless Aircraft Developments ...................................... 26

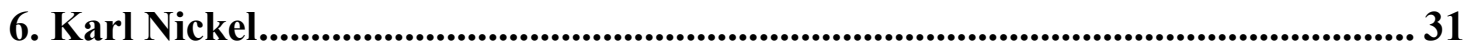

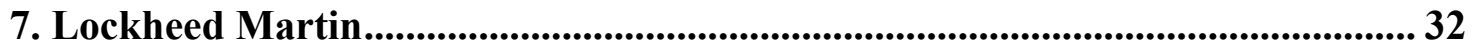

Chapter 2 Wing Morphing............................................................................................... 34

1. Early Aircraft Morphing Developments.............................................................. 34

2. NASA Mission Adaptive Wing (MAW) .................................................... 34

3. NASA Active Aero-elastic Wing (AAW)............................................................. 35

4. NASA Biologically Inspired Aerodynamic Geometries.................................... 36

5. DARPA / Lockheed MAS ....................................................................... 37

6. University of Florida................................................................................................... 39

7. Current Morphing Component Technology ......................................................... 40

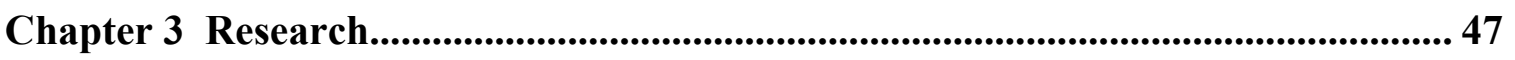

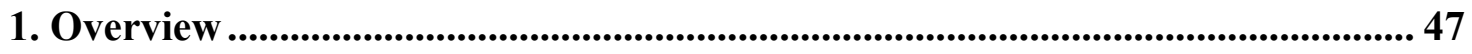

2. Objectives..................................................................................................................... 47

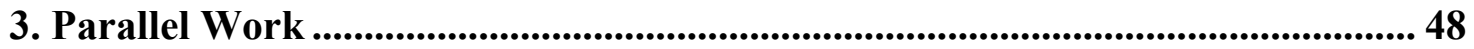

4. Geometry / Aerodynamics................................................................................... 49

5. Morphing mechanism development ..................................................................... 50

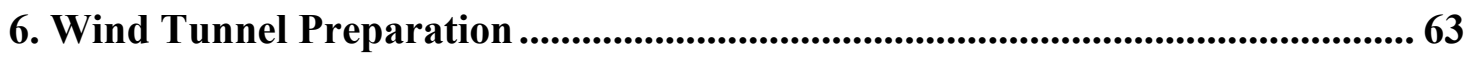

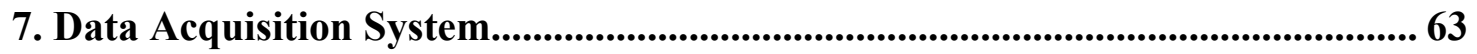


8. Force and Moment Balance System .............................................................................. 64

9. Wind Tunnel Test Case ................................................................................. 67

10. Wind Tunnel Testing ............................................................................. 72

11. Computational Fluid Dynamic studies...................................................... 84

12. Flight testing of the adaptive washout morphing mechanism ...................... 105

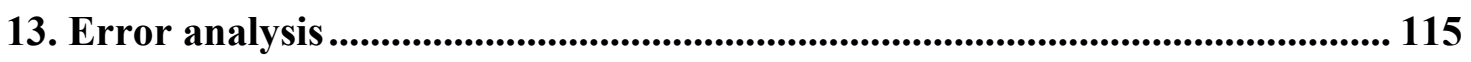

14. Discussion of Results........................................................................................ 121

Conclusions....................................................................................................................... 131

Bibliography ........................................................................................................... 133

Appendix A - Force Balance Data .................................................................................. 137

Appendix B - Wind Tunnel Data ................................................................................ 140

Curriculum Vitae .......................................................................................... 146 


\section{List of Figures}

Figure 1- Span-wise lift distributions, Bell shaped $\sin ^{3} v$, compared with elliptical and

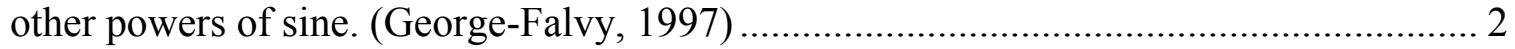

Figure 2 - Horten PUL-10 during flight-testing in Germany 1997. (Bullard, 2005) ........ 3 Figure 3 - The Zanonia seed, which inspired many to research tailless flight. The seed average 10-15 cm in span and glide long distances from their parent trees in Java. (Wahl,

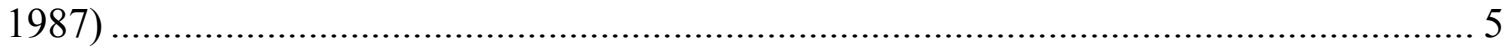

Figure 4- Lippisch and Opel's rocket powered Ente. (Bullard, 2001) ........................... 7

Figure 5- The Lippisch DFS 40. (Bullard, 2002) ......................................................... 8

Figure 6- The DFS 194 powered by a Walter "cold" Rocket already............................. 9

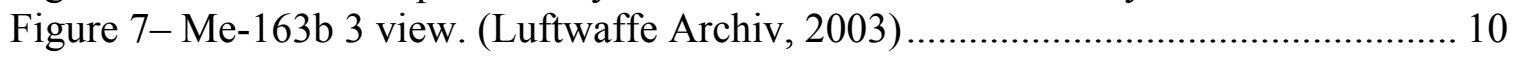

Figure 8- Me-163b gliding back from a mission (Reddin, 2003) ................................. 11

Figure 9- Me163b taking off note the obvious washout in the wing (Wainfan, 2000)... 12

Figure 10 - Northrop N9M in Flight (Bullard, 2004) ................................................... 14

Figure 11- The XB-35 in Flight (Bullard, 2005)...................................................... 15

Figure 12 - Horten Ho-II, Ho-III, Ho-IV and Ho-VII of 1942 Germany.(Mythra, 1998) 17

Figure 13 - A photograph of the Horten IX V2 during a test flight in 1944 or 1945

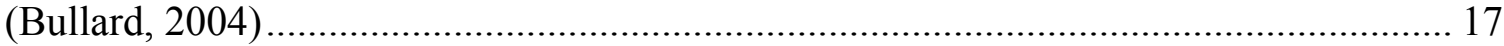

Figure 14 - Horten IX V1 unpowered glider test aircraft. (Bullard, 2002)..................... 18

Figure 15 - Horten XIII high sweep angle test aircraft and an artists depiction of a Horten

X Bring in picture of Horten X (Bullard, 2004) ....................................................... 19

Figure 16 - Horten XVc in Argentina (Bullard, 2003) ................................................ 19

Figure 17- Horten-Panek PUL-10 in flight over Germany in 1987 (Bullard, 2003) ....... 22

Figure 18 - The Horten IV during flight testing at Mississippi State University. The unaltered Ho IV with original landing skid can be seen on the left and the right shows the fairing built during testing in an attempt to improve the aircrafts performance. (Bullard,

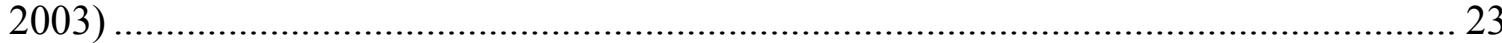

Figure 19 - Schematics of the Ho IV's controls, airfoil geometry and wash out geometry made by George-Falvy. Notice the Horten's use of the three pairs of elevons to achieve

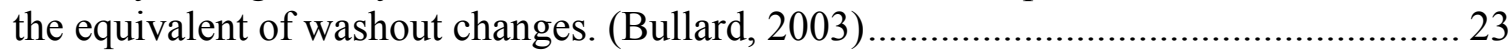

Figure 20 - Induced wingtip thrust due to wing twist and washout ............................... 24

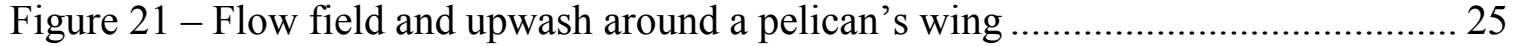

Figure 22 - Schematic of the SB 13 (Akaflieg Braunschweig, 2003)........................... 27

Figure 23- The SWIFT off the coast of Northern California and a 3-View Schematic.

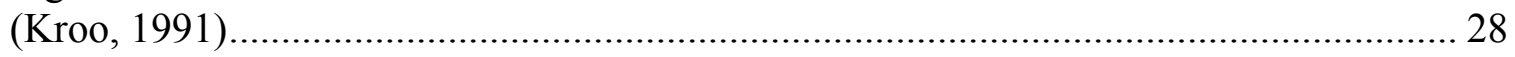

Figure 24 - Artists impression of the Boeing BWB (NASA, 1997) ............................... 29

Figure 25 - Boeing Blended Wing Body schematic (NASA, 1997) .............................. 29

Figure 26.-.The 17 foot and 6 foot BWB flight demonstrators. (Kroo, 1995).................. 29

Figure 27 - Artists Impression of the proposed Boeing 797.

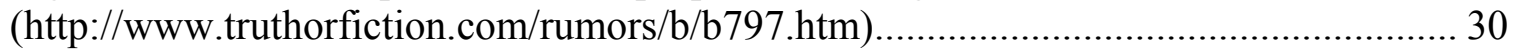

Figure 28- The graceful B-2 bomber in flight (Sweetman, 1999) ................................ 31

Figure 29.-.The Nickel Falter utilizing flexible skins and variable washout for primary control of a swept wing tailless aircraft (Nickel, 2005)............................................ 32

Figure 30 - Lockheed Polecat high altitude UAV which will in the future use wing morphing for 
control.(http://www.lockheedmartin.com/wms/findPage.dsp=fec\&ci=17787\& $\mathrm{rsbci}=0 \& \mathrm{fti}$

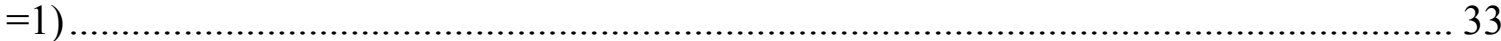

Figure 31 - The Wright Brother's 1899 wing warping design (NASM, 2004)............... 34 Figure 32 - The USAF-NASA F-111A MAW in flight at Dryden Flight Research Center

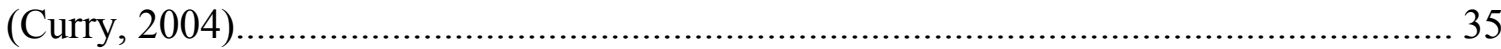

Figure 33 - F-18 AAW in flight and F-18 AAW schematic. (Curry, 2005) .................... 36

Figure 34 - Hyper-elliptic wing wind tunnel model (Lazos, 2002) .............................. 37

Figure 35 - The Lockheed Martin Tailless morphing demonstrator (Marks, 2004) ....... 38

Figure 36 - The first Lockheed Martin (MAS Project) Tailless morphing (Folding wing)

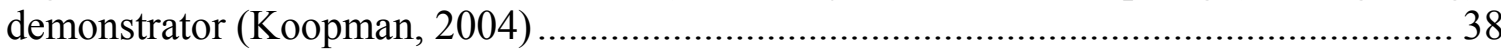

Figure 37- Cutaway of Lockheed Martin MAS UAV (Koopman, 2004) ....................... 38

Figure 38- University of Florida 24" "twisting" morphing demonstrator. ( Garcia, 2003)

Figure 39 - University of Florida 12" "curl" morphing demonstrator. (Garcia, 2003)... 40

Figure 40 - The strain versus voltage curve and displacement performance for a

THUNDER device (Simpson, 1998) ........................................................................ 42

Figure 41 - Inspiration for the Penn State-Darpa Compact Hybrid Actuators Program

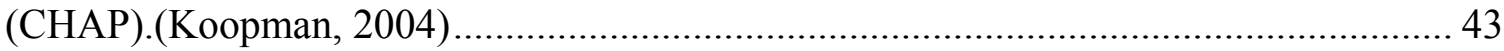

Figure 42 - Penn State CHAP Piezo-actuators (Koopman, 2004) ................................. 43

Figure 43 - Penn State CHAP Piezoactuator Load, Power velocity curves (Koopman,

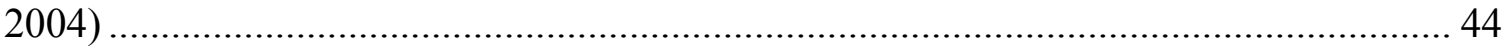

Figure 44- Smart composite wing model (Monner, 2002) .......................................... 45

Figure 45 - Final basic aerodynamic Non-morphing configuration with dimensions in inches and sectional washout angles................................................................. 50

Figure 46- First generation actuator and torque rod geometry for morphing control..... 51

Figure 47 - First generation composite skin made of woven glass and carbon and unidirectional carbon. 52

Figure 48 - WVU 1st Generation $610 \mathrm{~mm}$ span wind tunnel model in blue, showing

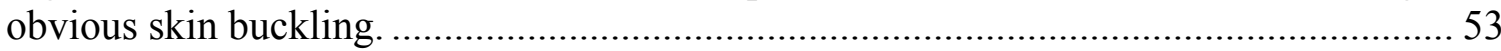

Figure 49 - Partially assembled second generation morphing wing showing the ribs,

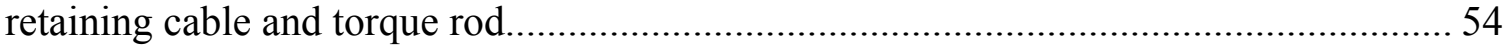

Figure 50- Morphing wind tunnel model showing the latex skin, the tensioning

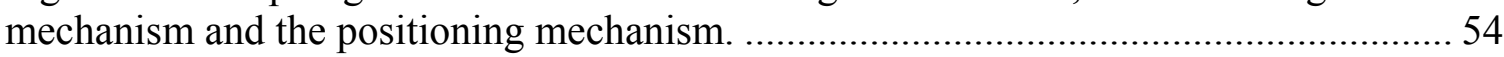

Figure 51-Completed morphing and non-morphing wind tunnel models..................... 55

Figure 52- Control position 3 in the morphing wing which corresponds to a +7 degree

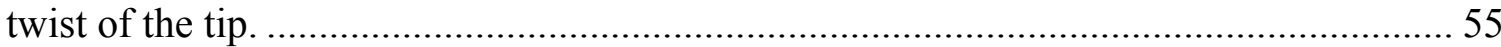

Figure 53- Control position 3 as seen on the elevon equipped comparison wind tunnel

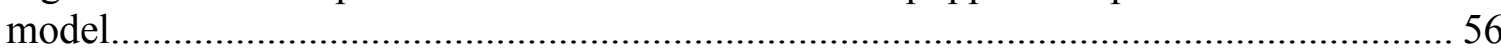

Figure 54- Comparison of initial model elevon deflection angle and initial Morphing wing model tip rotation angle for the 5 test control positions. .........................................56

Figure 55- Morphing wing trailing edge deflections for the 5 test control positions....... 57 Figure 56- Morphing wind tunnel model installed in the Vigyan wind tunnel in Hampton Virginia.

Figure 57- Early Elevon equipped wind tunnel model control forces and moments at 5 test control positions. 
Figure 58-Early Morphing wind tunnel model control forces and moments at 5 test

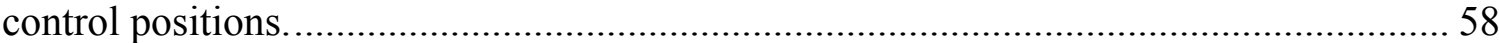

Figure 59 - Basic modified adaptive wash-out mechanism structure. ........................... 59 Figure 60- Spring 2006 modifications to the adaptive washout mechanism which include rounding of trailing edges, new Nylon restraining monofilament and Teflon spacers

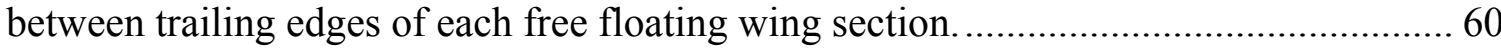

Figure 61- Skinned morphing wing, Yellow 0.050 inch monofilament, 0.014 inch Neoprene skin and control position adjustment mechanism can be seen. 60

Figure 62- Trailing edge curvature with in Nylon monofilament. Note Leading edge

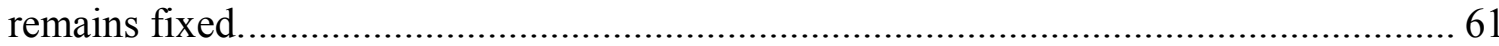

Figure 63 - Schematic of side view of morphing wing at control positions $1-5 \ldots \ldots \ldots . . .62$ Figure 64 - Elevon equipped wing mounted on force balance at 7 degrees AOA. The force balance mounting and 5 position control adjustment can be seen clearly.............. 62 Figure 65- The West Virginia University MAE subsonic closed loop wind tunnel, constructed in 1945 and capable of 200 fps flows in good condition....

Figure 66- Data acquisition computer interface mounted on a new shielded and grounded

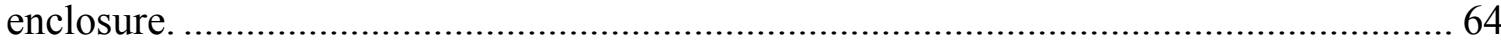

Figure 67 - Schematic of NASA Force Balance \#826 ............................................. 65

Figure 68 - Example of the calibration curves for a known lift load for the calculation of

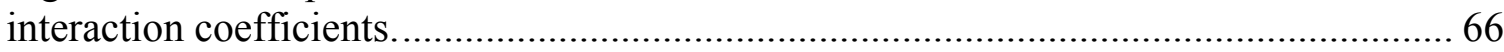

Figure 69- Six Component interaction load calculation work sheet for NASA balance interaction matrix as setup in WVU wind tunnel. Light green cells show new axial interaction coefficients.

Figure 70- Gerontakos and Lee's experimental setup to study tip vortices and then to

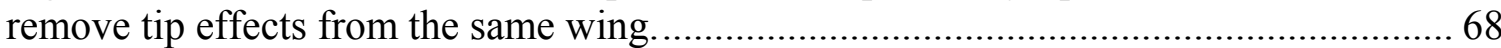

Figure 71-Coefficient of drag data from the 2006 Gerontakos and Lee study. ............. 69

Figure 72- Coefficient of lift data from the 2006 Gerontakos and Lee study.

Figure 73 - Validation Wind tunnel Model, NACA 0015, AR-2.5, 11 "cord for

$\mathrm{Re}=181000$

Figure 74 - WVU NACA 0015 validation model in the horizontal variable AOA mount

during smoke flow visualization. 70

Figure 75- Coefficient of lift data comparison between WVU NACA 0015 validation model data, Gerontakos data and 2D Re 160000 data from Sandia National Labs.... 71 Figure 76- Coefficient of drag data comparison between WVU NACA 0015 validation model data, Gerontakos data and 2D Re 160000 data from Sandia National Labs......... 71 Figure 77- Wind tunnel velocity, density and Reynolds number calculation worksheet. 73 Figure 78- Experimental aerodynamic coefficients for the elevon equipped wing at 7

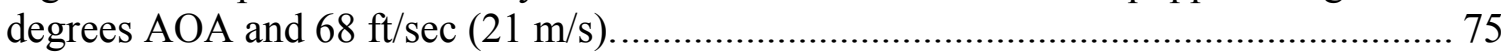
Figure 79- Experimental aerodynamic coefficients for the orphing wing at 7 degrees

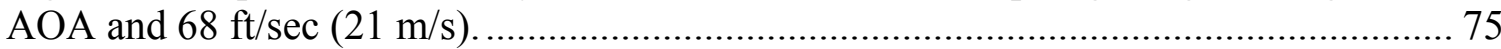
Figure 80- Experimental $C_{L}$ and $C_{D}$ aerodynamic coefficients for both wings at 0 degrees AOA and $68 \mathrm{ft} / \mathrm{sec}(21 \mathrm{~m} / \mathrm{s})$. 76 Figure 81- Experimental $C_{L}$ and $C_{D}$ for both wings at 7 degrees AOA and $68 \mathrm{ft} / \mathrm{sec}(21$ $\mathrm{m} / \mathrm{s})$.

Figure 82- Experimental L/D ratios for both wings at 7 degrees AOA and $68 \mathrm{ft} / \mathrm{sec}(21$ $\mathrm{m} / \mathrm{s}$ ). 
Figure 83- Experimental Yaw moment coefficients for both wings at 0 and 7 degrees

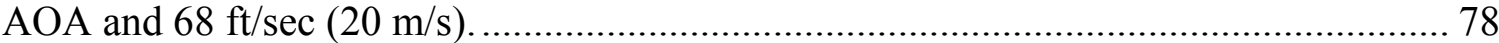
Figure 84- Experiments with a 2 watt laser sheet and glycerin smoke. Notice the smoke rake and transparent cylinder model.

Figure 85 - Elevon Wing at $68 \mathrm{ft} / \mathrm{sec}(21 \mathrm{~m} / \mathrm{s}), 7$ degree AOA and with a +3.5 degree control deflection 2 . 80

Figure 86- Elevon Wing at $68 \mathrm{ft} / \mathrm{sec}(21 \mathrm{~m} / \mathrm{s}), 7$ degree AOA and with a +7 degree control deflection.

Figure 87- Elevon Wing at $68 \mathrm{ft} / \mathrm{sec}(21 \mathrm{~m} / \mathrm{s}), 7$ degree AOA and with a -3.5 degree control deflection.

Figure 88- Elevon Wing at $68 \mathrm{ft} / \mathrm{sec}(21 \mathrm{~m} / \mathrm{s}), 7$ degree AOA and with a +7 degree control deflection. Note disturbed flow at the root of the elevon. 82 Figure 89- Morphing Wing at $68 \mathrm{ft} / \mathrm{sec}(21 \mathrm{~m} / \mathrm{s}), 7$ degree AOA and with an approximate -2 degree control deflection. Note lack of tip vortex..... 82

Figure 90 - Florescent oil flow visualization at $21 \mathrm{~m} / \mathrm{s}$ with rectangular wing using

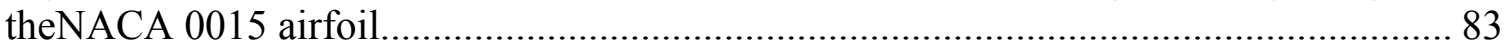

Figure 91- Non-morphing wing with florescent flow visualization fluid at 7 degrees AOA, control position 5 and $21 \mathrm{~m} / \mathrm{s}$.

Figure 92-Wing with florescent flow visualization fluid at 7 degrees AOA, control

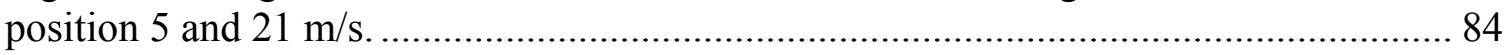

Figure 93-Pressure field measured by Chow and modeled by Dacles and Mariani......... 85

Figure 94- ONERA M6 Wing mesh used in NASA Ames CFD validation study........... 86

Figure 95- Validation Grid used on the Kim and Rhee type model. ............................... 87

Figure 96- Initial morphing wing mesh 1.6million cells............................................. 87

Figure 97- CFD validation model pressure field from the NACA 0012 Laminar model

which utilized a static pressure gradient adaptive grid at 2.17 million cells. .................. 89

Figure 98 - Morphing wing model in CFD test section generated in GAMBIT............... 90

Figure 99 - Morphing wing model mesh quality analysis. ............................................... 90

Figure 100 - Typical convergence monitors for an initial morphing wing model............ 91

Figure 101 - Tip of the morphing wing model before and after the use of Fluent's

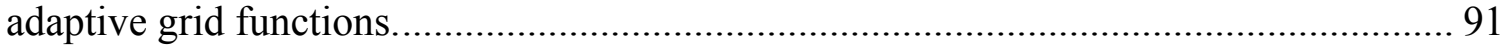

Figure $102-C_{L}$ convergence with pressure gradient adaptive grid ................................. 91

Figure 103 - Coefficient of Lift Comparison between a CFD model using Spalart-

Allmaras turbulence model and a Laminar flow CFD model at zero angle of attack. ..... 92

Figure 104 - Coefficient of Drag Comparison between a CFD model using Spalart-

Allmaras turbulence model and a Laminar flow CFD model at zero angle of attack. ..... 93 .Figure 105- Coefficient of Lift Comparison between a CFD model using SpalartAllmaras turbulence model and a Laminar flow CFD model at 7 degrees angle of attack.

Figure 106 - Coefficient of Drag Comparison between a CFD model using SpalartAllmaras turbulence model and a Laminar flow CFD model at 7 degrees angle of attack.

Figure 107 - Velocity magnitude vectors at the wing tip and wing static pressures at zero angle of attack and neutral control position. 95

Figure 108 - Velocity magnitude vectors at the wing tip and wing static pressures at zero angle of attack and a 7 degree control deflection. 95 
Figure 109 - Velocity magnitude vectors near the wing tip at 7 degrees angle of attack and a 3.5 degree control deflection, both Laminar and Spalart Allmaras models........... 96 Figure 110 - Velocity magnitude vectors near the wing tip at 7 degrees angle of attack and a 7 degree control deflection, both Laminar and Spalart Allmaras models. 96 Figure 111 - Static pressures and flow velocity vectors at cross sections in the tip region at 7 degrees angle of attack and a 3.5 degree control deflection, both Laminar and Spalart Allmaras models, $2 \mathrm{~cm}$ (distance fromtip), $7 \mathrm{U}$ (7 degrees up, LAM (laminar), SA (Spalart-Allmaras). 97 Figure 112 - Static pressures on the wing top at 7 degrees angle of attack and a -7 degree control deflection. 98 Figure 113 - Static pressures on the wing bottom at 7 degrees angle of attack and a -7

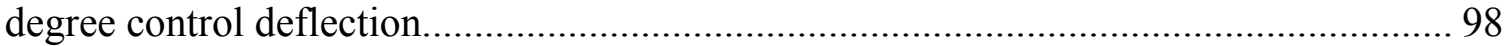
Figure 114 - Static pressures on the wing top at 7 degrees angle of attack and a -3.5

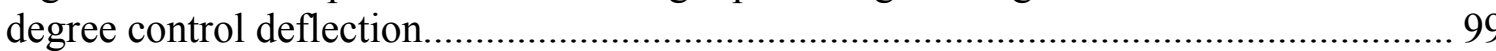
Figure 115 - Static pressures on the wing bottom at 7 degrees angle of attack and a -3.5 degree control deflection....................................................................................... 99 Figure 116 - Static pressures on the wing top at 7 degrees angle of attack and a 0 degree control deflection. 100 Figure 117 - Static pressures on the wing bottom at 7 degrees angle of attack and a 0

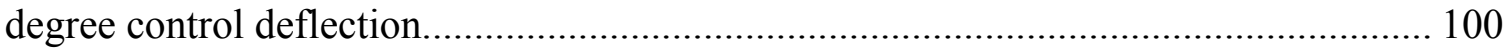
Figure 118 - Static pressures on the wing top at 7 degrees angle of attack and a +3.5

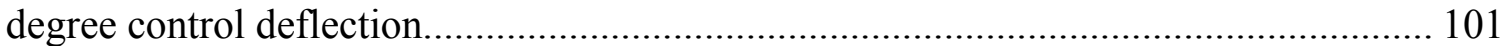
Figure 119 - Static pressures on the wing bottom at 7 degrees angle of attack and a +3.5

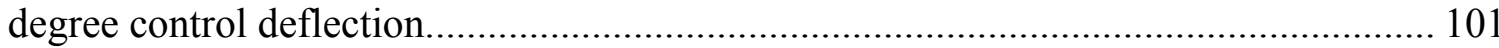
Figure 120 - Static pressures on the wing top at 7 degrees angle of attack and a +7 degree control deflection.

Figure 121 - Static pressures on the wing bottom at 7 degrees angle of attack and a +7 degree control deflection. 102 Figure 122 - Example of one of the planes generated to study the wake pressures and flows. 103

Figure 123 - Typical rapid dissipation of low pressure core structure down stream of the tip. By 1 inch behind the tip trailing edges the structure has mostly disappeared......... 103 Figure 124 - Low pressure core structures at the wing tip at the -7 degree control deflection (left) and the -3.5 control deflection (right). 104 Figure 125 - Low pressure core structures at the wing tip at the zero degree control deflection.

Figure 126 - Low pressure core structures at the wing tip at the +3.5 degree control deflection (left) and the +7 control deflection (right). Figure 127 - Results of wing loading study of production sweptwing tailless aircraft. . 105 Figure 128 - Full scale lower skin mold with Rohacell cores and glass/balsa ribs in

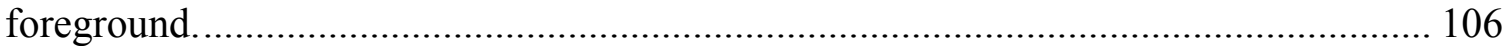
Figure 129 - Female molds with the upper skin bonded to the carbon cuff. ................. 107 Figure 130.-. Morphing mechanism component details ........................................... 108 Figure 131.-.Details of a single outboard Feather.................................................... 109 Figure 132 - Morphing mechanisn without tip feather, both the kynar coated carbon hinge rod and the $\mathrm{Al} /$ carbon actuator rods can be seen. 
Figure 133 - Completed morphing wing section covered by polypropolene and laytex skin.

Figure 134 - Servo and actuator arm through accesses panels in bottom od aircraft..... 111

Figure 135 - The Habicht II hooked up for data download. .......................................... 112

Figure 136 - Eagletree control software with realtime test flight capabilities................ 112

Figure 137 - Habicht II ready for flight with controls in trim position............................ 113

Figure 138- The Habicht I in its first northward turn and on overhead shot during stall tests.

Figure 139 - Example Altitude and propeller RPM data collected from flight testing. . 115 Figure $140-\mathrm{CD}, \mathrm{CL}$ and $\mathrm{L} / \mathrm{D}$ at increasing Re for the Elevon equipped wing at 7 degree AOA and in control position 5 .

Figure 141 - Experimental Drag Coefficient for Axi-Symmetric bodies. (Adapted from L. Prandl, "Ergebnisse der Aerodynamischen Versuganstalt zu Gottingen," p29, R.

Oldenbourg, Munich and Berlin, 1923; and F. Eisner, "Das Wiederstandproblem", Proc.

$3^{\text {rd }}$ Internatn. Congr. Appl. Mech, p 32, 1930.

Figure 142 - Wing sweep and twist and their effect on local AOA (Kuhlman, 2002) .. 123

Figure 143 - Outboard aileron and the rotation of the lift vector on a twisted swept wing tailless aircraft - (Kuhlman, 2002)

Figure 144 - Coefficient of Lift Comparison between wind tunnel data, a CFD model using Spalart-Allmaras turbulence model and a Laminar flow CFD model at zero angle of attack

Figure 145 - Coefficient of Drag Comparison between wind tunnel data, a CFD model using Spalart-Allmaras turbulence model and a Laminar flow CFD model at zero angle of attack.

Figure 146 - Coefficient of Lift Comparison between wind tunnel data, a CFD model using Spalart-Allmaras turbulence model and a Laminar flow CFD model at 7 degrees angle of attack.

Figure 147 - Coefficient of Drag Comparison between wind tunnel data, a CFD model using Spalart-Allmaras turbulence model and a Laminar flow CFD model at 7 degrees angle of attack.

Figure 148 - Low pressure core structures at the wing tip at the +3.5 degree control deflection (left) and the +7 control deflection (right).

Figure 149 - Morphing Wing at $20 \mathrm{~m} / \mathrm{s}, 7$ degree AOA and control position of approximately -2 degrees. Note the apparent lack of tip vortex.

Figure 150 - Morphing Wing Tip 7 degree AOA and control position of +7 degrees or +14 degrees to the wind tunnel flow, yet regionally this angle is much less due to upwash.

Figure 151 - Static pressure field on top wing surpface and near the tip at a +7 degree control deflection with the wing at 7 degrees angle of attack.

Figure 152 - Static pressure and velocity magnitude flow vectors just above the wing tip

surface. 130

Figure 153 - The common swifts use of LEVs and the swifts wing geometry. (Weiss, 2004)

Figure 154 - NASA Balance \#826 Calibrations, Drag Component. 137

Figure 155 - NASA Balance \#826 Calibrations, Vertical Component 137

Figure 156 - NASA Balance \#826 Calibrations, Lift Component. 138 
Figure 157 - NASA Balance \#826 Calibrations, Roll Component................................ 138

Figure 158 - NASA Balance \#826 Calibrations, Yaw Component ............................... 139

Figure 159 - NASA Balance \#826 Calibrations, Pitch Component................................ 139

\section{List of Tables}

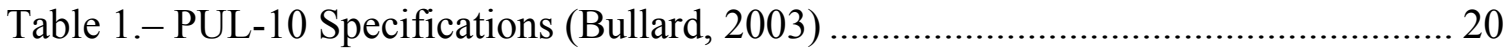

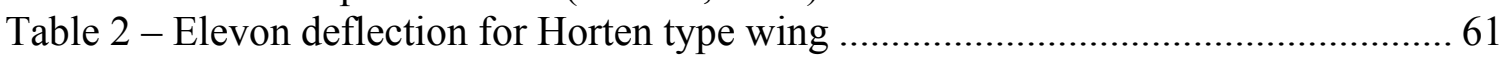

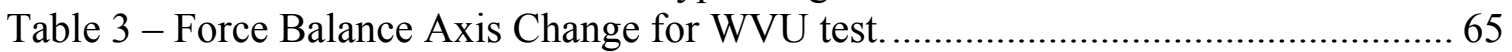

Table 4- Experimental aerodynamic coefficients for the elevon equipped wing at 7

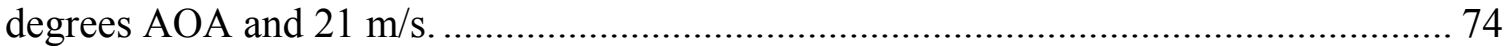

Table 5- Experimental aerodynamic coefficients for the Morphing wing at 7 degrees

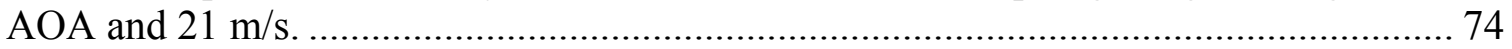

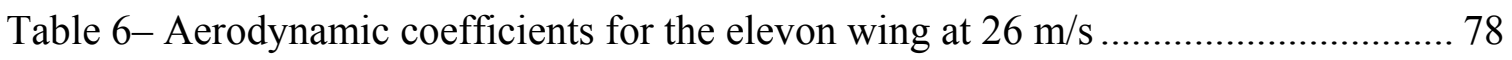

Table 7-Aerodynamic coefficients for the elevon wing at control position 5 and varying

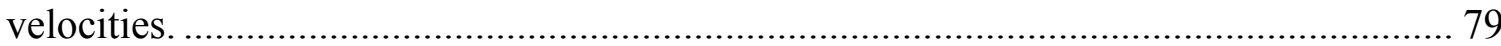

Table $8-\mathrm{C}_{\mathrm{L}}$ and $\mathrm{C}_{\mathrm{D}}$ of various Fluent models simulating Chows experiments ............... 88

Table 9 - Lift and Drag from Wind tunnel experiments $\left(\mathrm{C}_{\mathrm{D}} / \mathrm{C}_{\mathrm{L}}-\mathrm{WT}\right)$, Turbulent $\left(\mathrm{C}_{\mathrm{D}} / \mathrm{C}_{\mathrm{L}}-\mathrm{S}-\right.$

A)and Laminar CFD $\left(\mathrm{C}_{\mathrm{D}} / \mathrm{C}_{\mathrm{L}}\right.$-LAM)models at 0 and 7 degrees AOA. ......................... 92

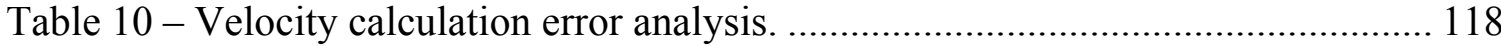

Table 11 - Force balance system error analysis. .......................................................... 119

Table 12- Coefficient of lift error analysis................................................................. 120

Table 13 - Coefficient of drag error analysis ............................................................. 120

Table 14- Pitching moment coefficient error analysis ............................................ 120

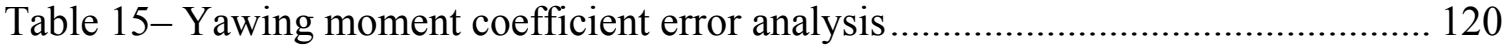

Table 16- Rolling moment coefficient error analysis................................................ 120

Table 17 - Aerodynamic Coefficient Data for Elevon Equipped Windtunnel Model in

WVU Wind Tunnel 2006-2007 .................................................................................... 140

Table 18 - Aerodynamic Coefficient Data for Morphing Windtunnel Model in WVU

Wind Tunnel 2006-2007 ....................................................................................... 143

Table 19 - Aerodynamic Coefficient Data for NACA 0015 Test Windtunnel Model in

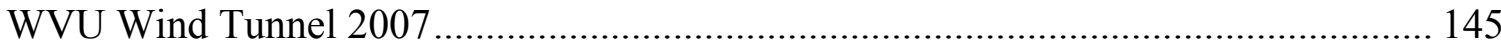




\section{Introduction}

When looking at the efficiency and elegance of bird flight in nature our aviation achievements seem to pale in comparison. Inspired by a gliding tree seed called Zanonia (Macrocarpa) and the flight of seagulls work done by Alexander Lippisch, Reimar and Walter Horten, and John Northrop offered a chance at achieving some of the efficiency of bird flight with swept wing tailless aircraft.

Swept wing tailless aircraft exhibit a highly efficient configuration with low parasitic drag, and up to $30 \%$ less total drag. A minimum of two control surfaces are needed for pitch, roll and yaw. Propulsion, fuel and payload are all built into the wing structure, which allows the design of a very lightweight rigid structure. (Northrop, 1947) Finally the smooth lines with very few protrusions allow the design of an aircraft with a minimum electromagnetic signature, which has become very important to today's military operations. When considering unmanned aerial vehicles (UAVs) a tailless aircraft not only has the above mentioned advantages but due to their geometry they can be easily folded into small containers for military or planetary exploration type missions.

Unfortunately, there are a number of design compromises necessary for tailless aircraft, which currently do not allow them to exploit many of their potential advantages.

A major aerodynamic/control problem with swept wing tailless aircraft is adverse yaw. Adverse yaw can be explained as a result of induced drag. When initiating a turn the elevon movement on the outboard wing increases lift and induced drag on that wing. This increases the adverse yaw, which drags the up-moving wing aft, opposite to the desired yaw direction. (Bowers, 1999) Alexander Lippisch recognized in the 1930's the effects washout (wing twist with the leading edge of the wing tip having a negative angle when compared to the root airfoil) had on adverse yaw. Lippisch originally used washout toward the wing-tips to reduce the risk of tip stalls, when he also observed a reduction in the effects of adverse yaw when maneuvering. Through the study of hundreds of model gliders and a large variety of tailless manned gliders and motor gliders, Reimar Horten developed the use of a bell shaped lift distribution for swept wing tailless aircraft as cure for adverse yaw in the 1930's. (Figure 1) Horten theorized that if you use a bell shaped lift distribution, instead of an elliptical lift distribution, adverse yaw would be minimized. This bell-shaped lift distribution is developed using wash-out of the wing. In a coordinated turn a down elevon position close to the wing tip increases the lift vector of the washed-out section of wing which effectively produces a small amount induced thrust at the wing tip negating the increased induced drag that causes adverse yaw. In theory this works, but there have been no formal studies which have confirmed this and Hortens own test pilots including Dr. Karl Nickel and Heinz Scheidhauer indicate that most of the Horten designs suffered from adverse yaw problems. Figure 2 shows Reimar Hortens final design the PUL-10. The flow physics in the tip region of a swept wing tailless aircraft are very complex and poorly understood with crossflow and a large upwash from relatively large root section of the wing. Recently researcher studying bird flight and bird formation

flight have observed induced thrust similar to that of the Horten designed wings. Other 
tailless aircraft designers have chosen to use winglets or up elevon on one side plus dihedral effect on the opposite to induce rolling and drag rudders for yaw as a solution, but many of these solutions produce additional drag.

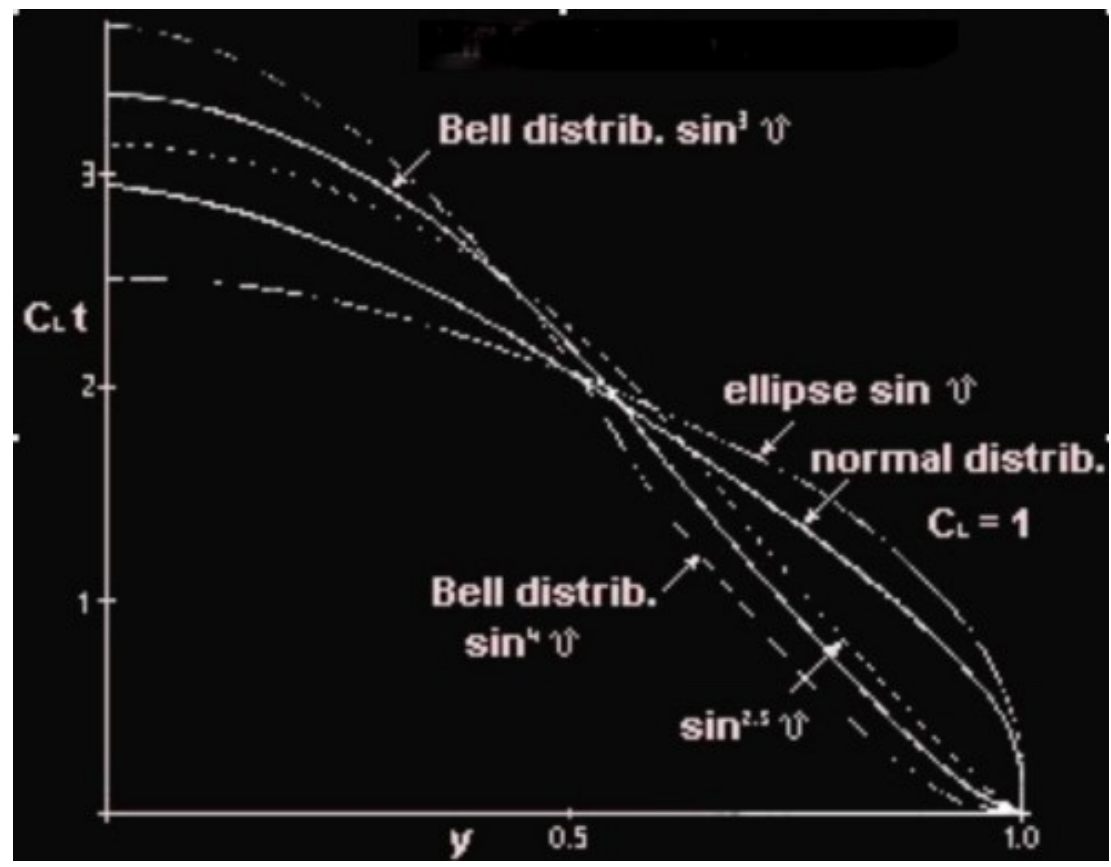

Figure 1- Span-wise lift distributions, Bell shaped $\sin ^{3} \dot{v}$, compared with elliptical and other powers of sine. (George-Falvy, 1997)

Tailless aircraft have a narrow Center of Gravity (cg) range. Any variations from design cg must be trimmed using control surfaces. Tailless aircraft that only use elevons for trim are susceptible to dangerous tip stalls. (risk minimized using built in wash out) Extreme elevon throws can initiate separated flow at the wing tips and cause a tip stall. This is also aggravated when elevons are used for trim for cg compensation.

In the late 1950's the Astrophysics Department of Mississippi State University and Dezo George Falvy conducted a thorough aerodynamic study of the Horten IV tailless sailplane. This study concluded that the aircraft could be redesigned to achieve a theoretical glide ratio of close to 50:1. Then many of the poor handling characteristics could be remedied if there was a way to adjust the washout in flight. (George-Falvy, 1997) Dr. Reimar Horten and Dr. Karl Nickel also came to a similar conclusion (Horten, 1993), The Hortens and Nickel also mathematically modeled elevon control inputs as effective washout. (Nickel, 1994) 


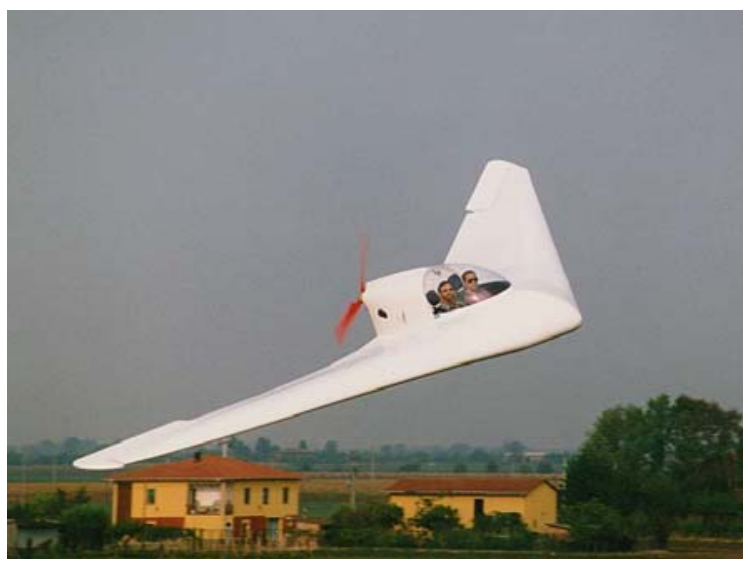

Figure 2 - Horten PUL-10 during flight-testing in Germany 1997. (Bullard, 2005)

Wing morphing technology offers us the opportunity to do away with many of the design compromises inherent for the control of swept wing tailless aircraft. Tailless aircraft will finally be able to approach the aerodynamic efficiencies envisioned by the pioneers in tailless aircraft. Enhanced control and maneuverability are also possible benefits of wing morphing.

Wing Morphing can immediately address the design compromise of using fixed washout or large winglets in swept wing tailless aircraft. Active morphing of the wing, variable washout, allows washout to be used only when maneuvering. This eliminates the corresponding increased drag for all non-maneuvering flight regimes.

\section{Chapter 1 Swept-wing Tailless Aircraft}

\section{Early Swept-wing Tailless Aircraft}

The 1920's and 1930's in Germany were a time of great innovation in aircraft and propulsion design. This is due to a few factors, post World War I politics, German government support and the coaching by old aeronautical geniuses.

During most of the 1920's Germany was prohibited from developing or manufacturing military aircraft. This led the aviation enthusiasts to sailplane and gliding competitions. The sailplane competitions encouraged quick and inexpensive development of new ideas. These competitions trained an entire generation of aeronautical engineers. The aerodynamic edge was constantly extended along with the development of lightweight building techniques. The competitions culminated on a 950-meter peak in the Bavarian Alps called Wasserkuppe. This was where the Olympics of gliding were held every year. This area was perfect for gliding, gentle grass covered slopes, which fell away into wideopen valleys. By 1937 there was a large complex of buildings catering to the gliding community. 
The rise of the NSDAP party and Germanys aspirations of regaining some of it's power and prestige, led the German government to whole heartedly support the education of aeronautics in schools and the official support of the sailplane competitions. Sponsorships and grants for innovative ideas became plentiful in the 1930's. (Ransom, 2001)

Aeronautical greats such as Prandtl, Ahlborn and Lilienthal trained or inspired this new generation of engineers competing at Wasserkuppe. Prandtl derived the basis for much of the fluid dynamic theory used today. Prandtl was very interested in the glider competitions and became friends with a young engineer named Alexander Lippisch who would develop the Me-163 and much of the theory of Delta wings. Prandtl along with Lippisch went on to coach the teenage Horten brothers who would go on to almost 60 years of tailless aircraft research. Professor Ahlborn, through his study of a gliding seed called Zanonia (Macrocarpa) (Figure 3) in the 1890's inspired many tailless aircraft designs. Ahlborn documented the hydrodynamics of this tailless flying seed and ran the German Hydrodynamic Institute into the 1920's. The Zanonia seed achieved stable flight by using a combination of swept wings with up to 10 degrees of washout at the tips. The washout at the tip provided a negative pitching moment, which countered the positive pitching moment of the center section. This information became the foundation for tailless aircraft theory developed by the Hortens and Lippisch as well as the British engineer John Dunne. Lilienthal who is Germany's equivalent to the Wright brothers conducted a number of manned glider flights in the 1890's also provided inspiration. 


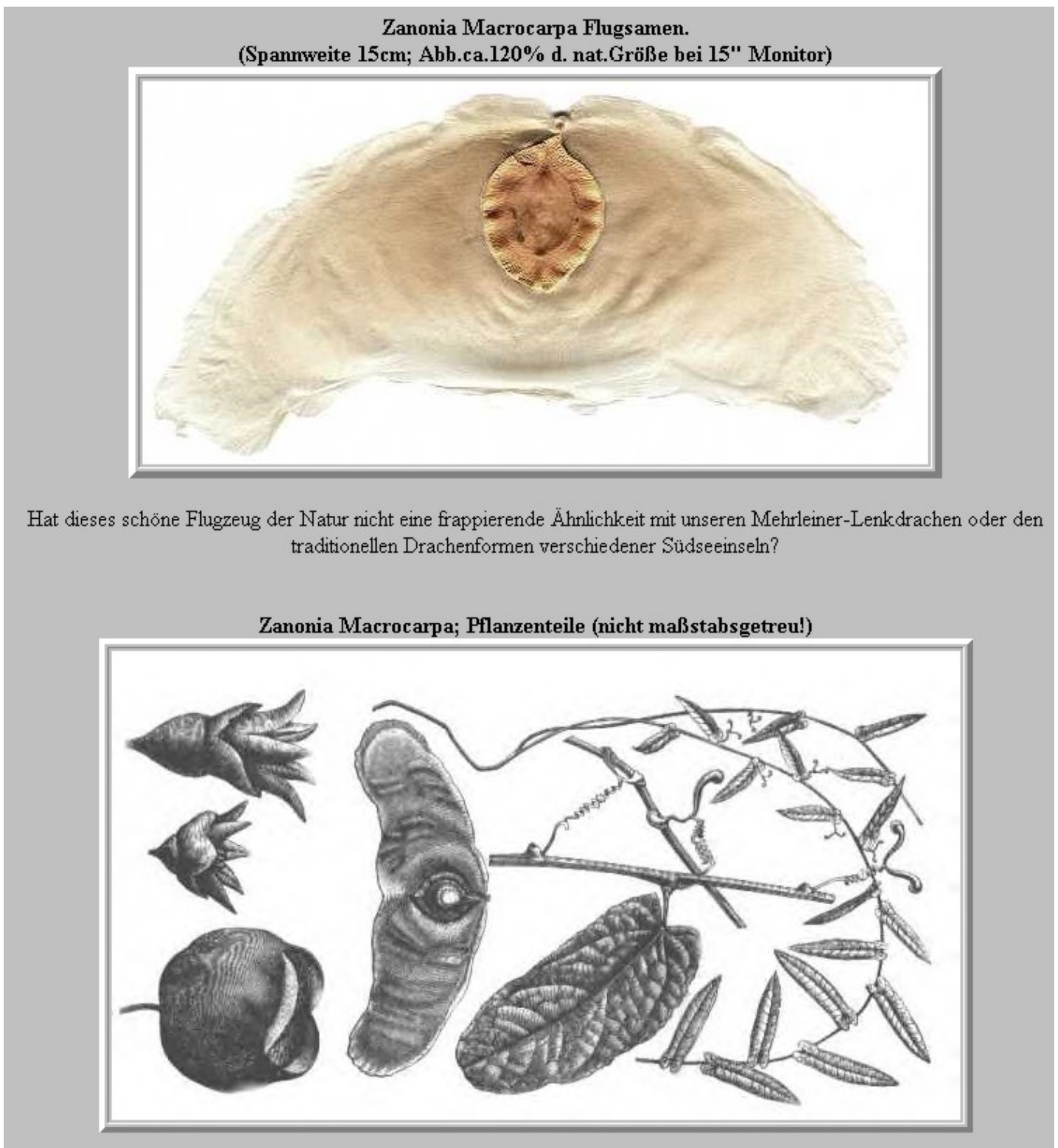

Figure 3 - The Zanonia seed, which inspired many to research tailless flight. The seed average 10-15 cm in span and glide long distances from their parent trees in Java. (Wahl, 1987)

\section{Alexander Lippisch}

As a boy of 14, Lippisch witnessed a flight by Orville Wright in September 1909. He followed the accounts of Dunne's and Etrich's experiments with inherent stability, and after military service during World War I, applied his interest to glider design. His first tailless glider was built in 1921, by Gottlob Espenlaub, the German glider enthusiast who would later collaborate with the Swiss designer Alexander Soldenhoff on his designs 
During the 20's, 30's and 40's Lippisch developed over 50 swept-wing, tailless designs. Many of Lippisch's designs were far from successful and at the time may have done more to illustrate the problems and instabilities inherent in tailless aircraft. These designs began the first serious, systematic development of tailless designs. In 1924, he became the Director of the Aeronautical Department of the RhonRossitten-Gesellschaft (RRG, which later became the German Research Institute for Soaring Flight).

Lippisch developed his designs with a methodology which would become common in 30 's and 40's Germany, testing the original concept first as a flying model, then as a man-carrying glider, and finally as a powered aircraft. Lippisch considered this approach would produce results in less time and with less expense than a wind tunnel research program. From this design philosophy evolved two famous series of tailless aircraft -- the Storch (stork) and the Delta.

In the late 1920's Lippisch developed the Storch series of flying wings. The ailerons of the late Storch models were redesigned to approximate the form of the Zanonia seed which created wash-out on Lippisch's swept flying wing. The Storch IV glider demonstrated impressive stability and control characteristics.

Although these experiments also met with moderate success, Lippisch returned to his original interests in 1929. These experiments, and subsequent research on the basic principles of rocket propulsion, provided the foundation for later projects with rocketpropelled aircraft in the late 1930s.

The Storch V took the lessons of the Zanonia seed and the Storch IV and combined them with a 8-Hp engine. Lippisch had this aircraft demonstrated at Tempelhof Airfield at Berlin in October 1929, with the expectation of obtaining some government financial backing, but none came. The early 1930's were difficult for Lippisch, because no funding for his research was available.

Rockets and aircraft came together when Lippisch was approached by Fritz von Opel. Opel a long supporter of rocket research asked Lippisch to adapt one of his Storch designs to utilize solid rockets. Development work on the Storch glider series was temporarily interrupted in 1928 when Lippisch, Fritz von Opel and the rocket manufacturer Sander developed and flew unmanned rocket powered gliders developed by Lippisch. These successful experiments were followed by a manned flight of a rocketpowered tail-less glider, the Ente (Figure 4), in September 1929. This aircraft was powered by 16 rockets of 50 pounds thrust each. With Opel at the controls the Ente made a 75 second, 2 mile flight. Although this project was a success Lippisch and Opel had a falling out so further research ended. 


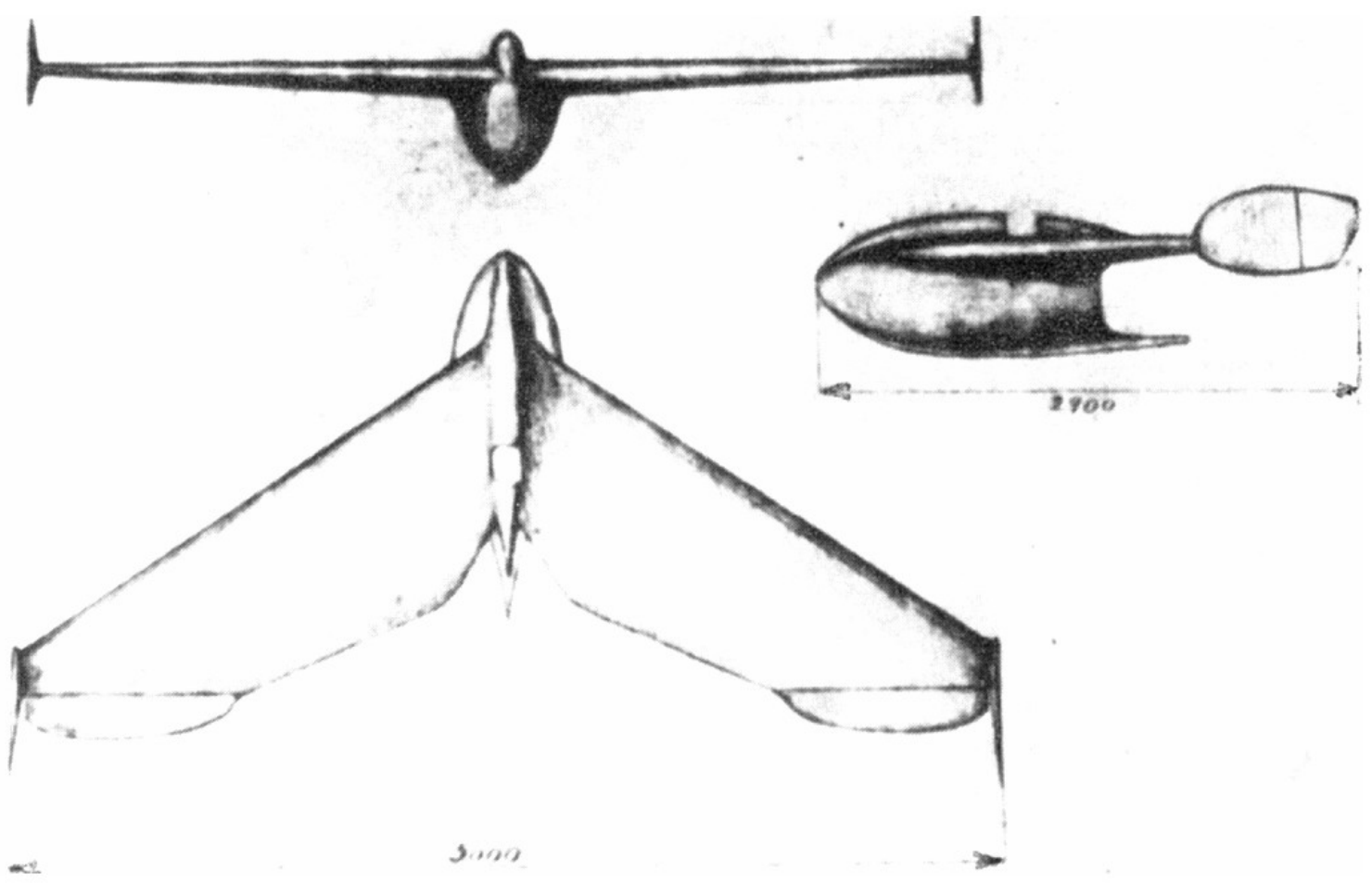

Figure 4- Lippisch and Opel's rocket powered Ente. (Bullard, 2001)

Lippisch continued his work after the Ente, first completing the Storch projects, with their swept back leading and trailing edges, then came the Delta, also a swept back wing but with one essential difference: the trailing edge, from wing tip to wing tip, was a straight line. This triangular wing allowed a thick midsection, with the potential for storing all loads inside the wing.

Lippisch proceeded from drawing to flying model to full-scale glider, and finally in June 1931, the powered Delta I was flown at the Wasserkuppe. Lippisch worked on many designs while head of the German Research Institute for Soaring Flight. Finally in 1939 the Government noticed the unique qualities of these aircraft and began to support these projects. The last two of this series were designated DFS 39 and the DFS 40.(Figure 5), (Bullard, 2001) 


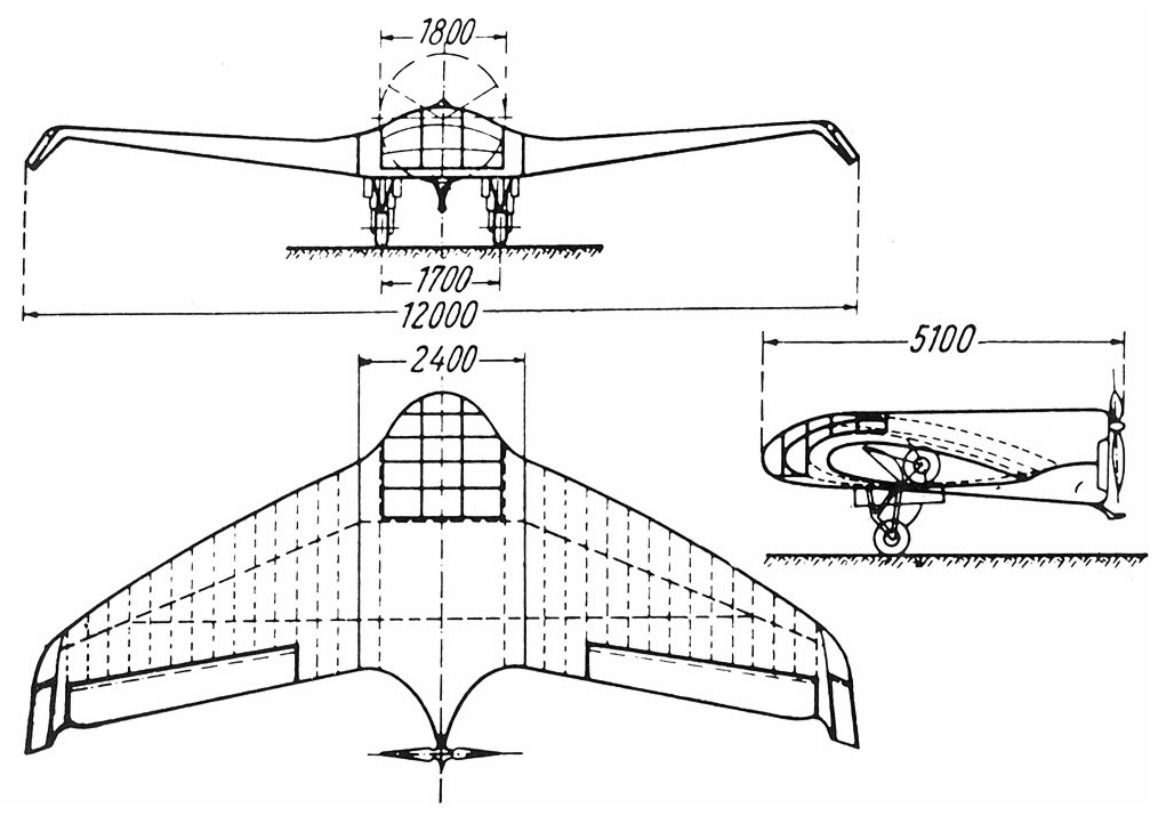

Figure 5- The Lippisch DFS 40. (Bullard, 2002)

The DFS 39 and 40 combined Lippisch's earlier ideas with his newer delta design. Their efficiency and flying qualities sparked the interest of many in the military who then approached Lippisch to design a tailless rocket powered glider (Projekt X, DFS 194) based on the DFS 40 and using the then new Walter hydrogen peroxide engines.

The DFS 194 was intended to fit a piston engine at first, but when Project $X$ and Lippisch's team came under the Government and Messerschmitt in 1939.

The DFS 194 was completed early in 1939 and ground tests with the Walter HWK R.1203 "cold" rocket motor began at Peenemünde in October of that year. Four months later the DFS 194 airframe was first test-flown in unpowered glider form. It was first flown under rocket power at Peenemünde in August 1940 with Heini Dittmar (Wasserkuppe glider pilot) flying it and subsequently made several highly successful flights, achieving $342 \mathrm{mph}(550 \mathrm{~km} / \mathrm{hr}$ ) in level flight. The DFS 194 (Figure 6) was a single-seat tailless aircraft similar in planform to the DFS 40 but lacking the wingtip anhedral, which was compensated for by the fitting of a larger central fin and rudder. The success of the flight test program led to the increase in the priority rating and funding of the Me 163 project. 


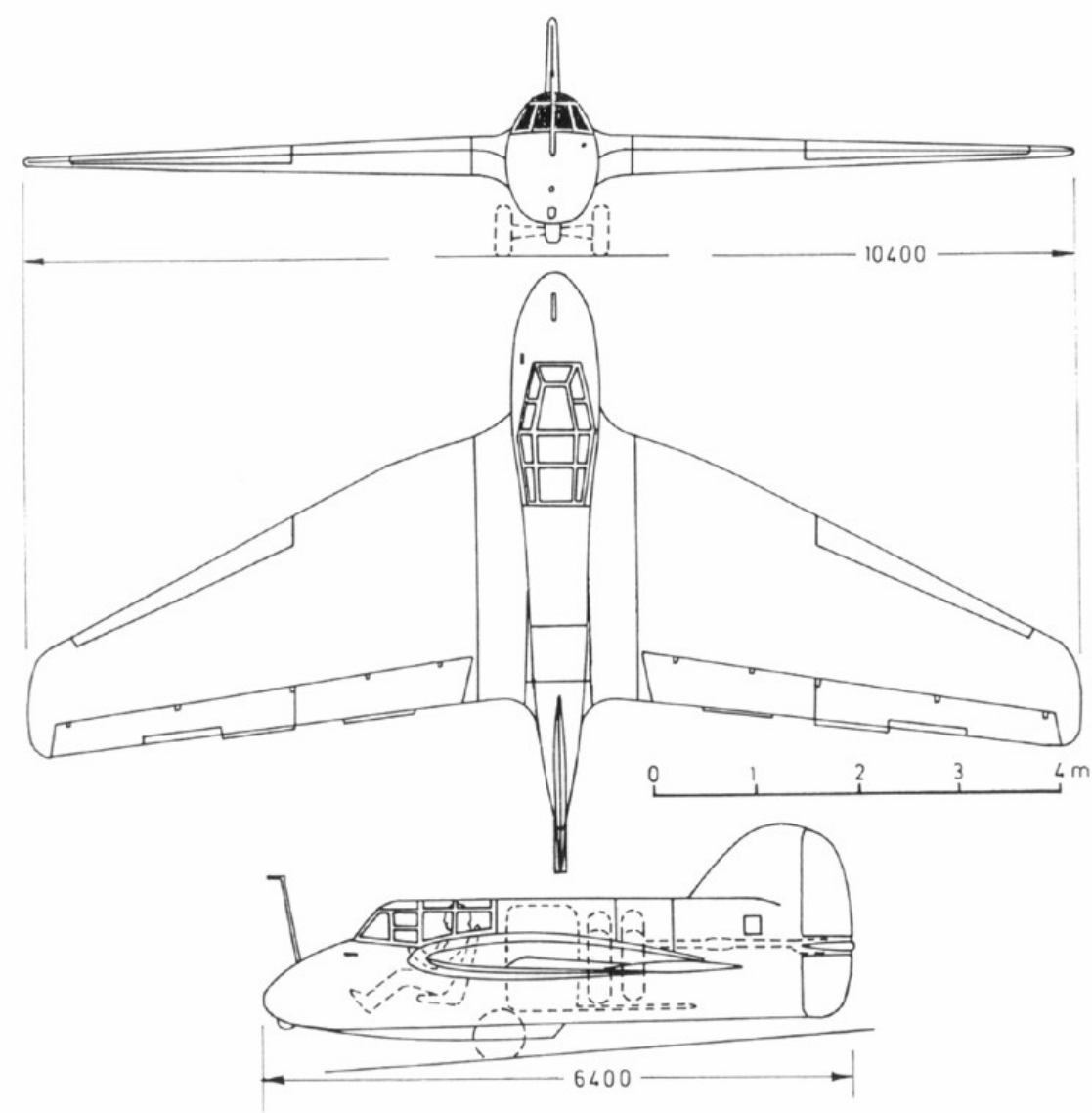

Figure 6- The DFS 194 powered by a Walter "cold" Rocket already looking very much like the Me-163. (Emmerling, 1992)

The first two Me 163A prototypes were completed in the spring of 1941. On October of that year, during the early powered flight program, a speed of over $623 \mathrm{mph}(1,003$ $\mathrm{km} / \mathrm{hr}$ ) was achieved and the Reich's Luftfahrt Ministerium (RLM- like NASA and DARPA together in the US) ordered the development of the Me 163 as a fighter.

Following a successful series of test flights with the DFS 194 at Peenemünde during 1940, Professor Lippisch's previously low-priority but highly secret Project X was speeded up. Three high-speed prototypes similar in layout to the DFS 194 were ordered. As the work had been moved to Messerschmitt from the DFS in early 1939, the three new aircraft were designated Messerschmitt Me 163.

After the first two prototypes, there followed a series of unpowered test flights during which a speed of $528 \mathrm{mph}(850 \mathrm{~km} / \mathrm{hr})$ was attained in a dive. In August 1941 the Me 163 had been fitted with a Walter HWK R.11-203 3301, 650 1b (150- $750 \mathrm{~kg})$ variablethrust rocket motor. On only the fourth flight with this powerplant a level speed of 570 mph $(920 \mathrm{~km} / \mathrm{hr})$ was attained, and on October 2, 1941, test pilot Heini Dittmar reached a speed of over $623 \mathrm{mph}(1,003 \mathrm{~km} / \mathrm{hr}$, Mach 0.85$)$ only a couple of minutes after casting 
off from the tow aircraft. The RLM immediately ordered the further development of the Me 163 interceptor. (Figure 7) The Me-163a was a great success but was not an operational fighter yet it needed better take-off performance, better range and a useful weapons load. In May of 1941 the ME-163b was ordered, which incorporated a fuel load $3000 \mathrm{lbm}, 2 \times 30 \mathrm{~mm}$ cannons, the Walter HK-109-509 "hot" engine.

Construction of the 163B was started on December 1, 1941. Seventy aircraft were ordered. The need for Walter submarine propulsion units and RATO units caused the priority level of the Me163b's engines to be lowered, which caused delays.

\section{Dreiseitenansicht Me 163 B}

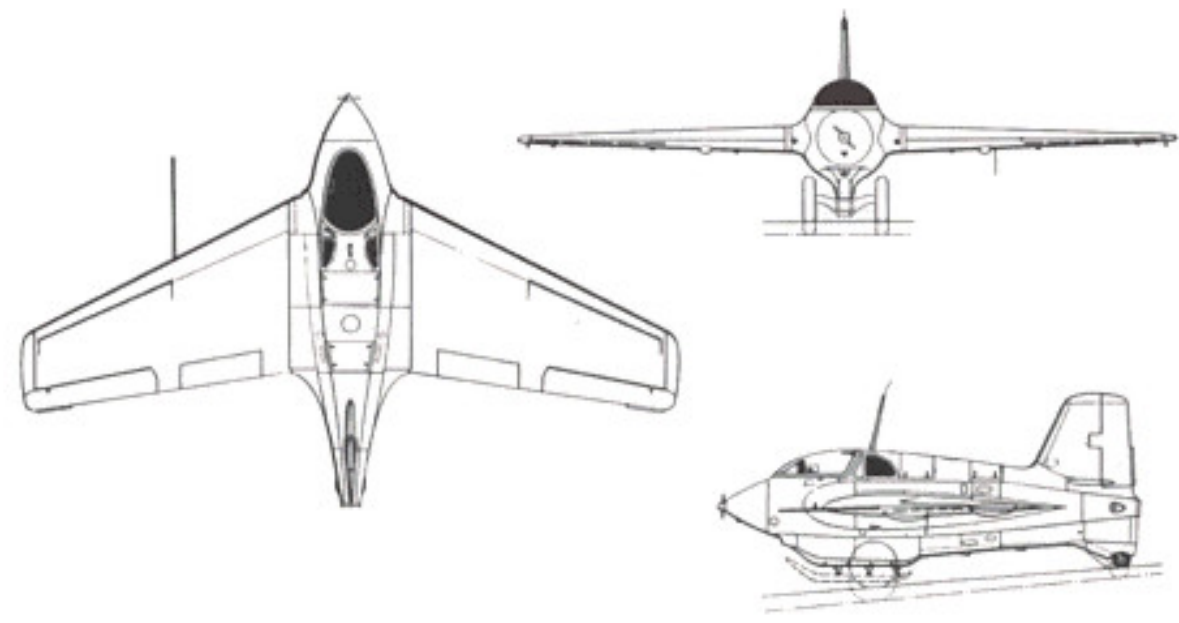

Figure 7- Me-163b 3 view. (Luftwaffe Archiv, 2003)

Many of the deficiencies found in the Me-163a had to be designed out of the Me-163b at the same time that full production was beginning. Landing gear and range were a major headache.

Short range would plague this program until it's end. It was originally intended that a Rocket Assisted Take Off (RATO) would be used when an aircraft was scrambled. This would conserve much of the onboard fuel for the mission radius. When such a strategy was used the Me163b would have a radius of action of near 50 miles. Due to shortage in man power and confusion during the war, a very low priority was given to assisted takeoff program and this therefore limited the Me-163b's radius of action to about 35 miles.

Aerodynamically, the Me 163b (Figure 8 \& Figure 9) was the combination Lippisch's experiments. By all accounts, its basic flying qualities were excellent. It was stable in all 
axes, and pilots praised its maneuverability and handling. Problems occurred when pilots transitioned into the Komet from conventional tailed aircraft, because many small mistakes in low speed flight could not be recovered from.

The airplane had a wing with a 25.8 degree swept-back and with 7 degrees of washout and no horizontal tail. A pair of fabric-covered elevons on the outer portions of the wings controlled both pitch and roll. The primary reason for the wing sweep was to create the pitch moment arm for the elevons rather than to increase the critical Mach number of the airplane.

A flap system was required because of the very low drag of the basic Komet airframe. The airplane was very clean aerodynamically, giving it a flat glide angle. This made it very difficult for a pilot to hit a precise touchdown point. The Komet also had a tendency to float in ground effect.

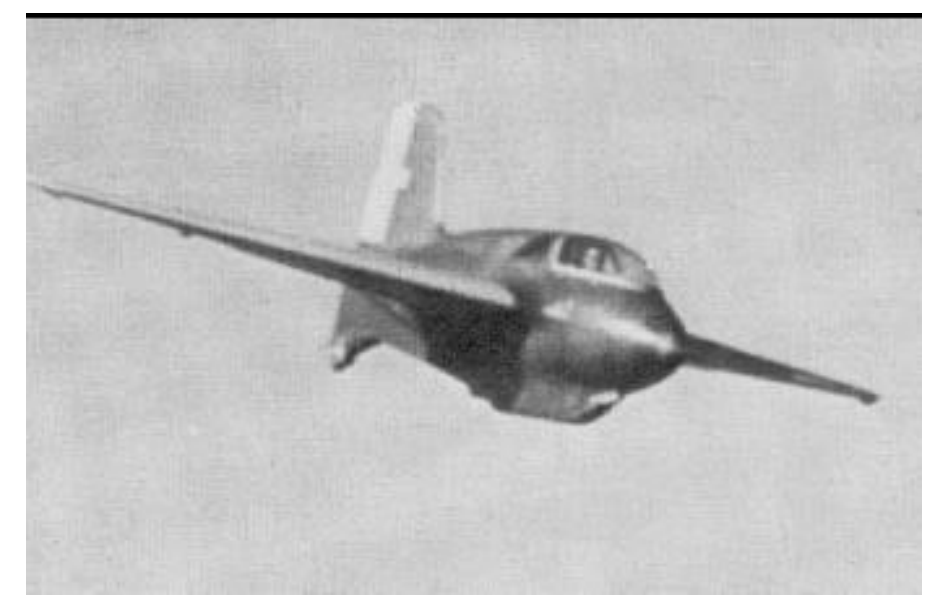

Figure 8- Me-163b gliding back from a mission (Reddin, 2003)

Adding a landing flap and/or aerodynamic drag brake to the airplane steepens the glide, making it easier for the pilot to hit his landing spot precisely. Putting flaps on the Komet was not simple. The small moment arm of the elevons made the use of conventional flaps impossible. The elevons were not powerful enough to trim out the nose-down pitching moment generated by flaps. So, the solution was to place landing flaps under the inboard half of the wings, with the hinge line well forward of the trailing edge. These could be deflected without affecting pitching moment. The craft also carried inboard, trailing-edge trim flaps.

The Komet was the first fighter consistently to have difficulties due to compressibility effects. As air flows over an object, it changes speed. Due to the Me-163b's clean lines and excess thrust it was capable of flying above the critical Mach number but didn't have the power to exceed the speed of sound, the aircraft could fly in the "transonic" speed range. The airspeed is less than Mach 1, but there are local areas of supersonic flow embedded in the overall areas of high curvature. The critical Mach number depends on 
the configuration of the airplane. The thicker the wing, the more the air accelerates when passing over it. Some of the early WW II fighters, notably the P-38 Lightning, began to run into some transonic aerodynamic effects at Mach numbers as low as 0.68 or 68 percent of the speed of sound. The Komet had a critical Mach number of about 0.84 .

When supersonic flow begins to appear on a wing or tail surface, the aerodynamic center moves aft, causing a nose-down pitching moment. As the Mach number increases, a shock wave forms at the aft boundary of the supersonic-flow bubble. When the shock gets strong enough it will cause the airflow to separate aft of the shock, leading to a loss of lift. This condition is called "shock stall."

On the Me 163, the combination of the aft shift in aerodynamic center and shock stall led to a dangerous condition known as "Mach tuck." If the Mach number exceeded approximately 0.85 , the airplane would begin to nose down on its own. The pilot would naturally react by pulling on the stick and deflecting the elevons upward. This would cause a shock wave to form on the underside of the wing at the elevon hinge line. The elevons would shock stall and be unable to bring the nose up, causing the airplane to pitch over into an ever-steepening dive. The only hope for recovery was to wait until the airplane had dived to a lower altitude where the speed of sound is higher, thus reducing Mach number, and the elevons would regain effectiveness. (Wainfan, 2000)

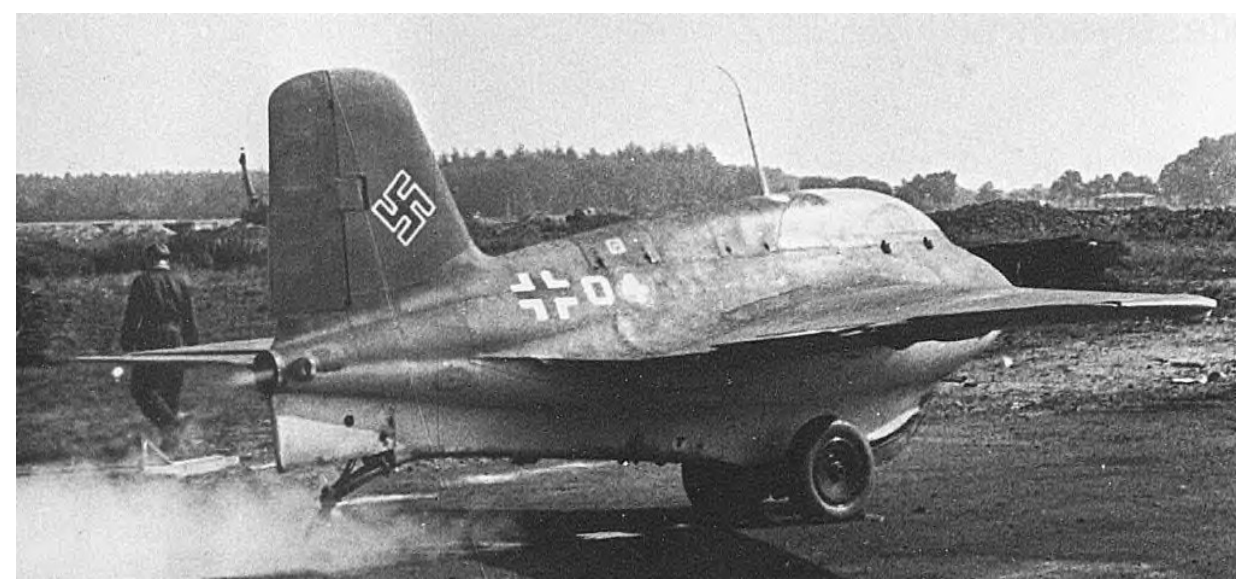

Figure 9- Me163b taking off note the obvious washout in the wing (Wainfan, 2000)

The first operational unit received a squadron of Me 163bs late in June 1944, and was first used operationally in July of that year. The first interception of American B-17 bombers followed on August 16, though none were shot down. Although 300 or so Komets were built, only around 11 successful attacks were recorded. This is mostly due to the fact that the closure rate was too high for the $30 \mathrm{~mm}$ cannons to be effective. The typical approach speed of the Komet was $590 \mathrm{mph}$ and the top speed of the bomber formations was $250 \mathrm{mph}$, which give a closure speed of $340 \mathrm{mph}$. The Mk $10830 \mathrm{~mm}$ cannon had an effective range of 650 yards and the pilot had to take evasive action at 200 
yards range. This gave the pilot 2-3 seconds to hit his target. Another factor was that the Komets operational career did not begin until the Allied bombing had devastated Germany. Fuel factories were destroyed and fuel transportation was almost impossible.

\section{John K. Northrop}

John Northrop realized the possible advantages of creating an all wing aircraft from the early 1920's on. Northrop in a presentation given in 1947 gives the following reasons for his interest in all wing aircraft. "The ratio of the minimum parasite drag coefficient (CDmin) for all-wing airplanes to that for conventional types is approximately 1:2. Minimum drag coefficients for a number of large bomber and transport aircraft, of the time, such as the B-29, B-24, C-47 and others average approximately .023. The minimum drag coefficients for several all-wing types have been measured both in model and fullscale configurations and vary from less than .010 to about .0113 , which is the figure for the XB-35 including armament protuberances, drive shaft housings, rudimentary nacelle for gun emplacements, and so on.

Under high-speed conditions with any type of power plant the parasite drag becomes a much larger percentage of the total drag than for cruising conditions with reciprocating engines. At high speed the parasite drag may account for 80 percent or more of the total, while the induced drag drops to 20 percent or less. Using an assumed figure of 80 percent parasite drag, which is probably correct to +10 percent for most aircraft, the power required to drive the all-wing airplane at the same speed as the conventional airplane will be from 40 percent to $18-1 / 2$ percent less and the range, at the high speed of the conventional airplane, will be from 66 percent to 22 percent greater, as indicated in. As turbojet and turboprop power plants both operate at relatively high speed for best fuel economy, the advantages of the all-wing configuration, when used in combination with these power plants, will closely approach the above figures for maximum range as well as high speed.

There are other major advantages of the all-wing type which cannot be so definitely evaluated but which can and do contribute appreciably to improvement in efficiency and range. Two of these, namely the elimination of jet-tail surface interference, and the possible elimination of wing-tail surface shock wave interference, have already been mentioned. The third, and the most immediately applicable to designs of the near future, is the improved adaptability of all-wing types to the distribution of major items of weight empty and useful load over the span of the wing. While such distribution can be made to a limited extent in conventional airplanes, it can be much more fully accomplished in the all-wing type. Such weight distribution results in substantial savings in structural weight, which have important effects on the ratio of gross weight at takeoff to landing weight. An analysis of the range formula indicates that this ratio is one of the most important range parameters. Competent authority has shown that distribution of fuel in the wings instead of the fuselage of a large conventional modern transport would allow an increase in gross 
weight of 16 percent without increase to weight empty, with a corresponding increase in range up to 30 percent.

The all-wing airplane provides comparative structural simplicity, plus the possibility of structural material distribution in a most effective way at maximum distances from the neutral axis, plus an opportunity to stow power plant, fuel and payload at desirable intervals along the span of the wing, which cannot be equaled in conventional types. These matters are rather intangible and difficult to illustrate by numerical relationships. They depend to a large extent on the type and size of the airplane, what it is designed to carry, and what the desired high speed may be." (Northrop, 1947)

Northrop's original ideas were incorporated into the N-1M (Northrop Model 1 Mockup) to explore the flight characteristics of a possible tailless bomber design. The $\mathrm{N}-1 \mathrm{M}$ was designed with removable wing sections so plan-form, sweepback, dihedral, tip configuration, C.G. location, and control surface arrangement could be changes between flights. This aircraft flew over 200 flights with numerous configurations.

One of the goals of this research was to eliminate vertical fin and rudder surfaces and get rid of their associated drag. Tip mounted split flaps were found to be the best replacement for a conventional rudder. The split flap was then combined with the trimming surface needed to counteract the moment generated by the landing flaps.

The lessons of the NM-1 were brought together in the four N-9M (Northrop Model 9 Mockup), (Figure 10) aircraft, which were to test this type of configuration for use in a larger bomber type of aircraft.

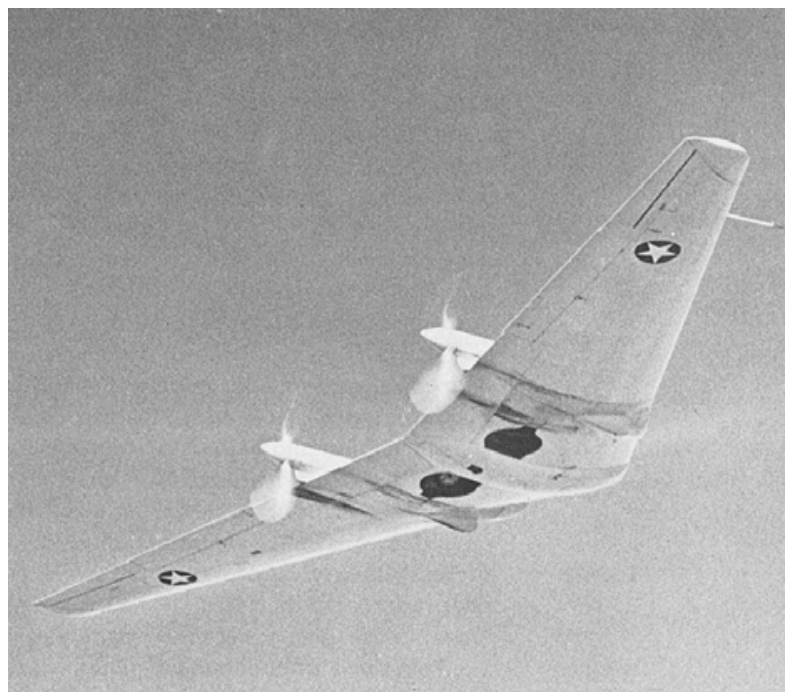

Figure 10 - Northrop N9M in Flight (Bullard, 2004)

The first M9M began flight-testing in December 1942. This aircraft was lost in a test flight due to an uncontrollable spin. Later studies indicated that the spin recovery parachutes and conventional techniques used in spin recovery were ineffective and that 
new techniques using aileron instead of elevator movement were superior. Hundreds of hours of tests in the N9Ms defined the limitations in $\mathrm{Cg}$ and maneuvering for this configuration.

These lessons then led to Northrop's ultimate tailless designs, which were the XB-35 and XB-49 long-range bombers. The first XB-35 (Figure 11) took it's first flight on June 25 1946. Engine / gearbox problems plagued the program and were never really solved until two of the XB-35s were converted to the YB-49 configuration by replacing the piston engines with eight 4000 pound thrust J35 engines.

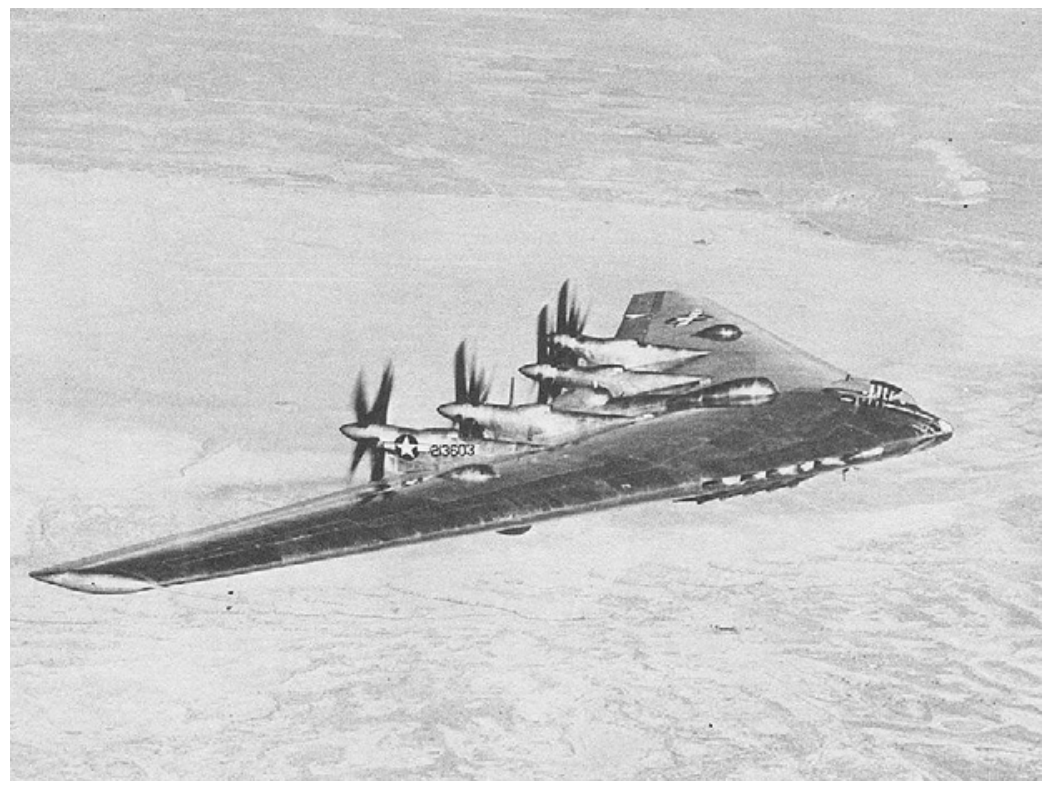

Figure 11- The XB-35 in Flight (Bullard, 2005)

First flight of the YB-49 was on October 21, 1947. During the testing program the second YB-49 crashed, killing all aboard, including copilot Captain Glenn Edwards, for whom Edwards AFB was named. This crash occurred during aft $\mathrm{Cg}$ tests. The testing continued until 1948 when the US Air Force awarded a contract for 30 reconnaissance versions. Another XB-49 was lost due to engine problems. The XB-49 exhibited a yawing oscillation, which made it difficult to direct bombs accurately. These factors along with and Northrop's political problems caused the contract to be withdrawn and all of the aircraft to be scrapped. (Bullard, 2003) Northrop's strategy for the control of a swept wing tailless aircraft was to use the middle span surfaces for pitch and roll, and the outboard surfaces to trim for pitch and split to act as drag rudders. This combination can easily lead to tip stalls which are very serious in a tailless aircraft. 


\section{Reimar and Walter Horten}

Reimar and Walter Horten were self taught tailless aircraft designers. Reimar initially gained his inspiration for tailless aircraft design while a teenager. Reimar and his brother Walter two year older, were always interested in flight. During a glider competition held by the Lower Rhine Union in 1927 a small rocket powered glider won first place after the rocket accidentally burned the back of the model off. Based on this experiment they began to refine their tailless designs. They entered and won the nation 1930, 1931 and 1932 national glider competitions at Wasserkuppe. They then decided that they had done all they can do with models and decided to take their designs up to full scale. They kept up on with the achievements of Alexander Lippisch and tried to incorporate and prove his theories with their own work.

In 1932, Reimar and Walter only 17 and 18 years old respectively designed and built the Horten I sailplane, based on their earlier glider experiences. The aircraft was competitive in the international competition at Wasserkuppe in 1934 and won a 600 mark prize for most original design.

In testing the Ho I the Horten's realized that much of this design could be refined. Support came from many people after their performance at Wasserkuppe, so the brothers had the materials they needed for the Horten II. Reimar worked on his own now because Walter was serving as a pilot in the Luftwaffe. In 1936 Reimar was drafted into the Luftwaffe and became a flight instructor in Cologne. The technical officer over Reimar, who was a sailplane enthusiast, saw the Horten II and decided that the Luftwaffe should have a sailplane team and should use Horten Designs. Reimar then had a workshop and craftsman at his disposal. In 1938, Reimar and Walter won the Lilienthal Prize in Aviation for their Horten II design. Reimar's theories continued to develop with powered and unpowered versions of the Horten III. They now had the opportunity for a formal education at the Technical University of Berlin. In 1937 the Hortens received a contract to build a twin engine aircraft, called the Horten IV to test some new plastic coverings and structural materials. The Second World War initially made things difficult for the Horten's work. Over time they learned to take advantage of the situation and started gathering the resources they need for their glider research. A modified version of the powered Horten III was put into production for training and as a small arms ammunition transport. Multiple Horten IIIs were constructed at various universities and for the military. Reimar developed his ultimate sailplanes between 1939 and 1941, the Ho IV and Ho VI for the 1941 Wasserkuppe competitions. (Figure 12). 


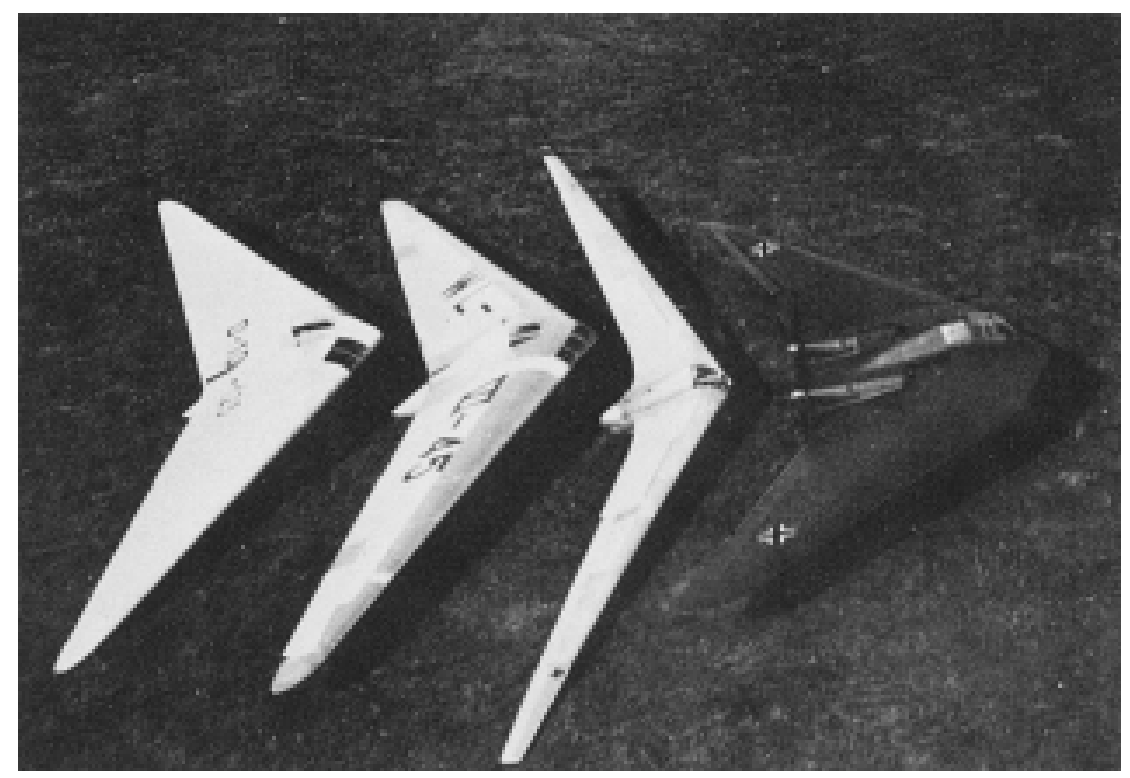

Figure 12 - Horten Ho-II, Ho-III, Ho-IV and Ho-VII of 1942 Germany.(Mythra, 1998)

They both had largest aspect ratios possible with the materials available. Walter, after being a fighter pilot in the Battle of Briton decided that a good tailless fighter could be what Germany needed. Walter also had a new position in the Luftwaffe, which was in the inspection office for aircraft research and production. Reimar's design theories matured and now Walter had the connections needed to get both official and unofficial support from the government. Using the Horten $\mathrm{V}$ as a stating point the developed the Horten IV which was a twin piston engined aircraft. While working on the Ho IV the Hortens witnessed Lippisch's Me-163 and learned of the new turbojet engines. The turbojet engine was what Reimar dreamed of for his aircraft. They had significant thrust at high speed and could be buried in the wing to reduce drag. They decided that the Ho VII would be used as a trainer for a new aircraft, the Ho IX, (Figure 13 \& Figure 14) which would use two Junkers JUMO-004 turbojet engines. Contracts were placed to manufacture the Ho VII.

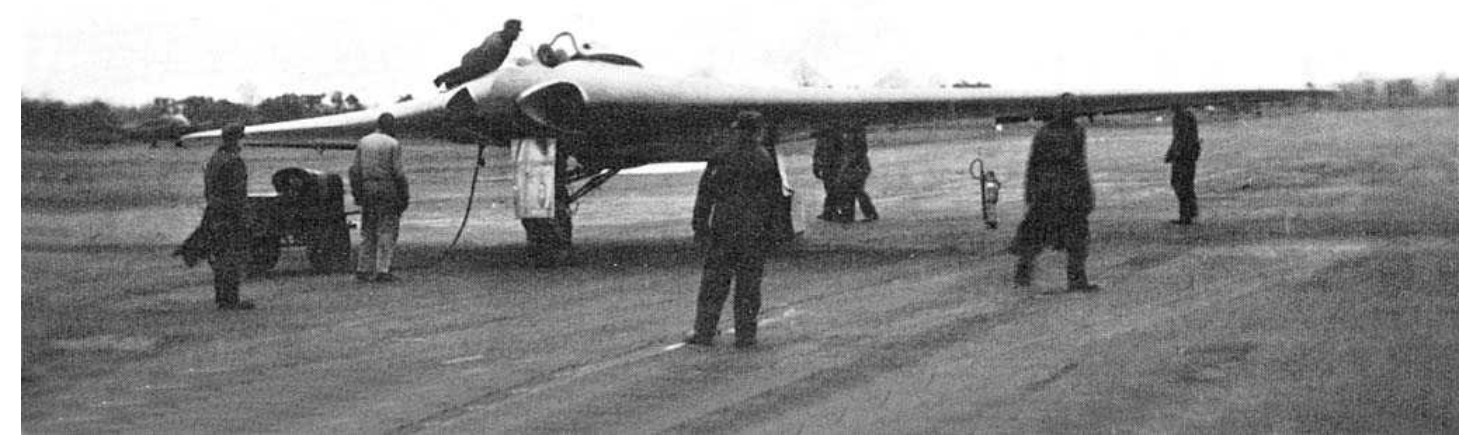

Figure 13 - A photograph of the Horten IX V2 during a test flight in 1944 or 1945 (Bullard, 2004) 


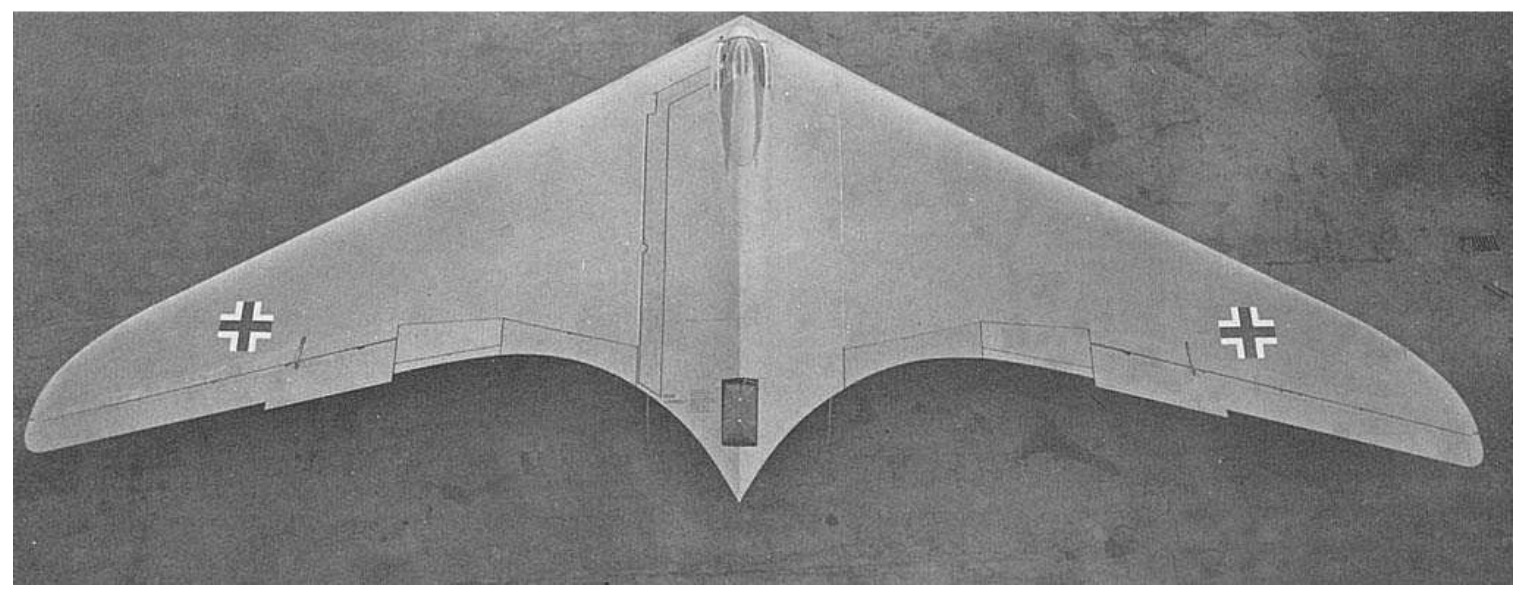

Figure 14 - Horten IX V1 unpowered glider test aircraft. (Bullard, 2002)

The Ho IX made its first flight in December 1944. Satisfied with the initial flight, in February 1945, the German government ordered 40 aircraft to be built. The Ho IX V-2 had flown three or four times before it crashed in February 1945. The Ho IX was returning to Oranienburg near Berlin when it reported an engine failure. The engine failure also caused a loss of the aircrafts hydraulics. An emergency compressed air system was used to extend landing gear and flaps, so they could not be retracted. The additional drag from the gear and flaps made it necessary to add more power on the good engine to maintain altitude. The asymmetrical thrust caused by the power increase was too much for the drag rudders and elevons to counter, so the power was reduced again and the aircraft crashed short of the landing field. The US Army captured the Ho IX V-3 intact and nearly completed, and also the V-4, V-5 and V-6 in various stages of completion at the Gotha factory and the Ho IX V1 near Leipzig. The H IX V-3 was later shipped to USA, and is now in the Smithsonian collection, awaiting restoration. (Bullard, 2004)

While Reimar worked on the Horten IX fighter he was also working on two important projects. The first was the Horten X supersonic fighter. (Figure 15) The Horten X was utilized highly loaded highly swept wings. The Horten aerodynamic theories were combined with highly swept wings for testing on a glider called the Horten XIII. Glider testing validated Reimar's ideas for using highly swept wing in a tailless configuration, but the war ended before this aircraft design was completed. The other important project was the Horten XVIII which was called the "Amerika Bomber". This aircraft utilized multiple turbojets to achieve highspeed and high altitude plus the low drag from the tailless configuration to reach America from Germany. 

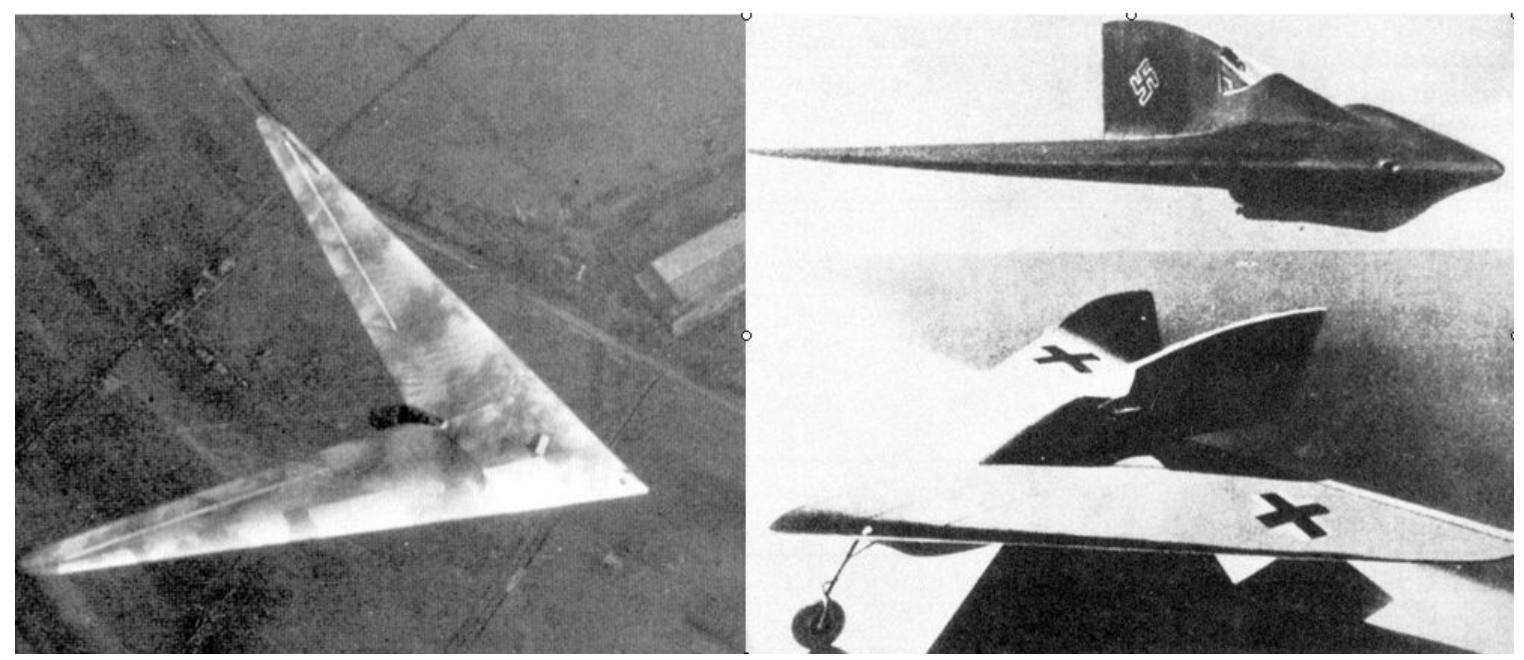

Figure 15 - Horten XIII high sweep angle test aircraft and an artists depiction of a Horten X Bring in picture of Horten X (Bullard, 2004)

After the war, Walter stayed in Germany and helped re-establish the German Airforce under NATO and Reimar went to Argentina to try to continue his work. In Argentina Reimar developed two jet fighters and a 120-foot span tailless transports aircraft. Reimar's real passion was in tailless sailplanes. He developed many sailplanes in the post war years, one of which was the first to cross the Andes (Mythra, 1998). After the over throw of Juan Peron Reimer government support for his work was lost, so he returned to sailplanes. His sailplane research in Argentina culminated in the development of the Horten Xc, a foot launched sailplane. Prior to the Horten Xc design, the Ae. 41 (Horten XVc) (Figure 16) was which was the first sailplane to cross the Andes on October 30th, 1956.

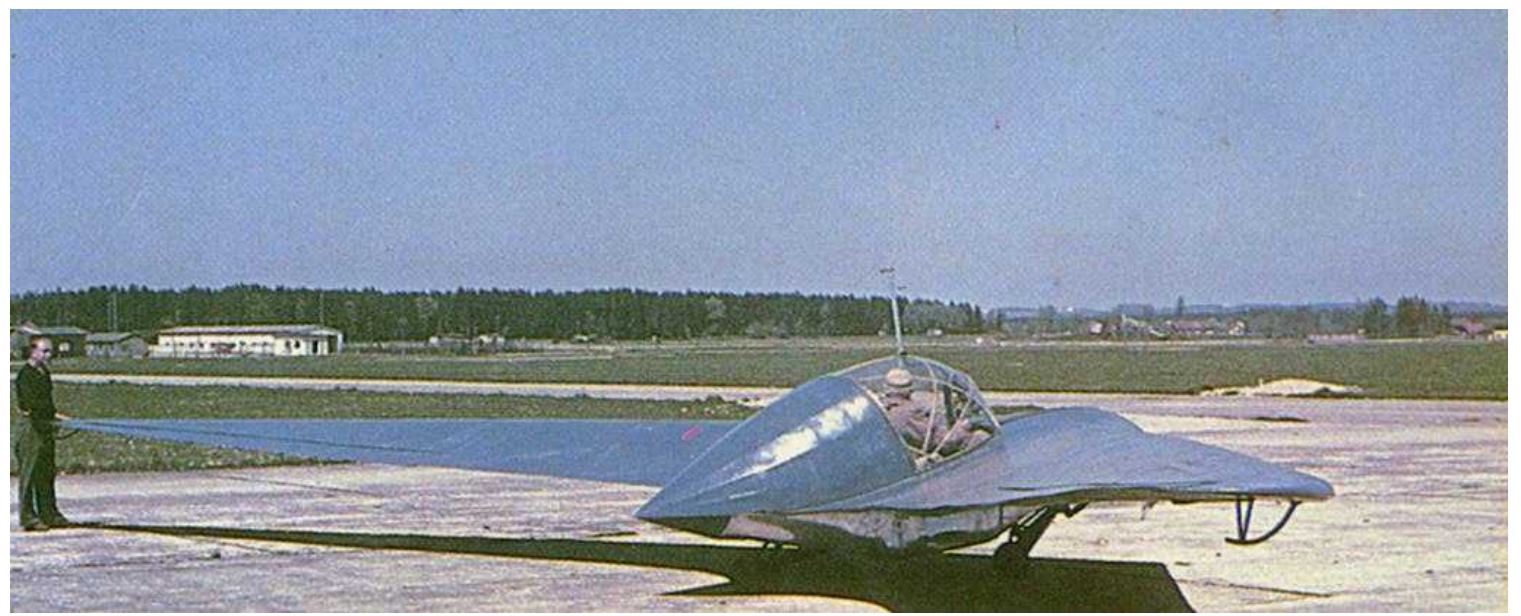

Figure 16 - Horten XVc in Argentina (Bullard, 2003)

In the winter of 1989-1990, Siegfried Panek, worked with Dr. Horten on the design of the PUL-9(Panek Ultra-Light 9 meter wing span), a light general aviation tailless aircraft. 
The PUL 9 was thoroughly tested under many different flight conditions. Handling and stability in the air were gentle and highly impressive. In 1991, Horten and Panek decided to design a twin place aircraft, which was to become the PUL-10. This aircraft used the Horten theories and modern composites. The PUL-10 began flight-testing in 1994. In 1995 the 2-stroke Rotax 582 was replaced with a 4-stroke Rotax 912.

A third PUL 10 prototype was completed in fall 1995, completed its flight tests in 1996 and received German flight registration in 1997. (Figure 17) Problems with the Rotax engines promoted a change to a $90 \mathrm{hp}$ BMW motorcycle engine. One prestigious organization, the Oskar-Ursinus-Association, awarded the constructors of the PUL 10 the first prize for the most progressive design in 1994 and 1997. (Bullard, 2003)

Table 1.- PUL-10 Specifications (Bullard, 2003)

\begin{tabular}{|c|c|c|}
\hline Designer & \multicolumn{2}{|l|}{ Dr. Reimar Horten } \\
\hline Name & \multicolumn{2}{|l|}{ Horten PUL 10} \\
\hline \multirow[t]{4}{*}{ Main Dimensions } & Wing Span & $10 \mathrm{~m} / 33 \mathrm{ft}$ \\
\hline & Total Length & $3.96 \mathrm{~m} / 13 \mathrm{ft}$ \\
\hline & Total Height & $1.43 \mathrm{~m} / 4.7 \mathrm{ft}$ \\
\hline & Wing Area & $15.42 \mathrm{~m}^{2} / 165.9 \mathrm{ft}^{2}$ \\
\hline Seats & \multicolumn{2}{|l|}{2} \\
\hline \multirow[t]{12}{*}{ Wing Geometry } & Tapered Wing (T.W.), Span & $10 \mathrm{~m} / 33 \mathrm{ft}$ \\
\hline & Root Chord Length (T.W.) & $2.5 \mathrm{~m} / 8.2 \mathrm{ft}$ \\
\hline & Root Chord, real & $2.875 \mathrm{~m} / 9.43 \mathrm{ft}$ \\
\hline & Tip Chord (T.W.) & $0.5 \mathrm{~m} / 1.6 \mathrm{ft}$ \\
\hline & Tip Chord, real & $0.0 \mathrm{~m}$ \\
\hline & Aspect ratio & 6.485 \\
\hline & Wing Sweep & $35^{\circ}$ \\
\hline & Wing Dihedral & $2^{\circ}$ \\
\hline & Wing Twist & $10^{\circ}$ \\
\hline & Root Airfoil Section, Horten (T.W.) & $d=18 \%, f=4 \%$ \\
\hline & Root Airfoil Section, Horten, real & $d=21 \%, f=4,66$ \\
\hline & $\begin{array}{l}\text { Tip Airfoil Section, similar NACA } 0010 \\
\text { (T.W.) }\end{array}$ & $d=10 \%, f=0 \%$ \\
\hline Outer Wing & Single main-spar & \\
\hline Center Section & Crash-stiff passenger nacelle & \\
\hline
\end{tabular}




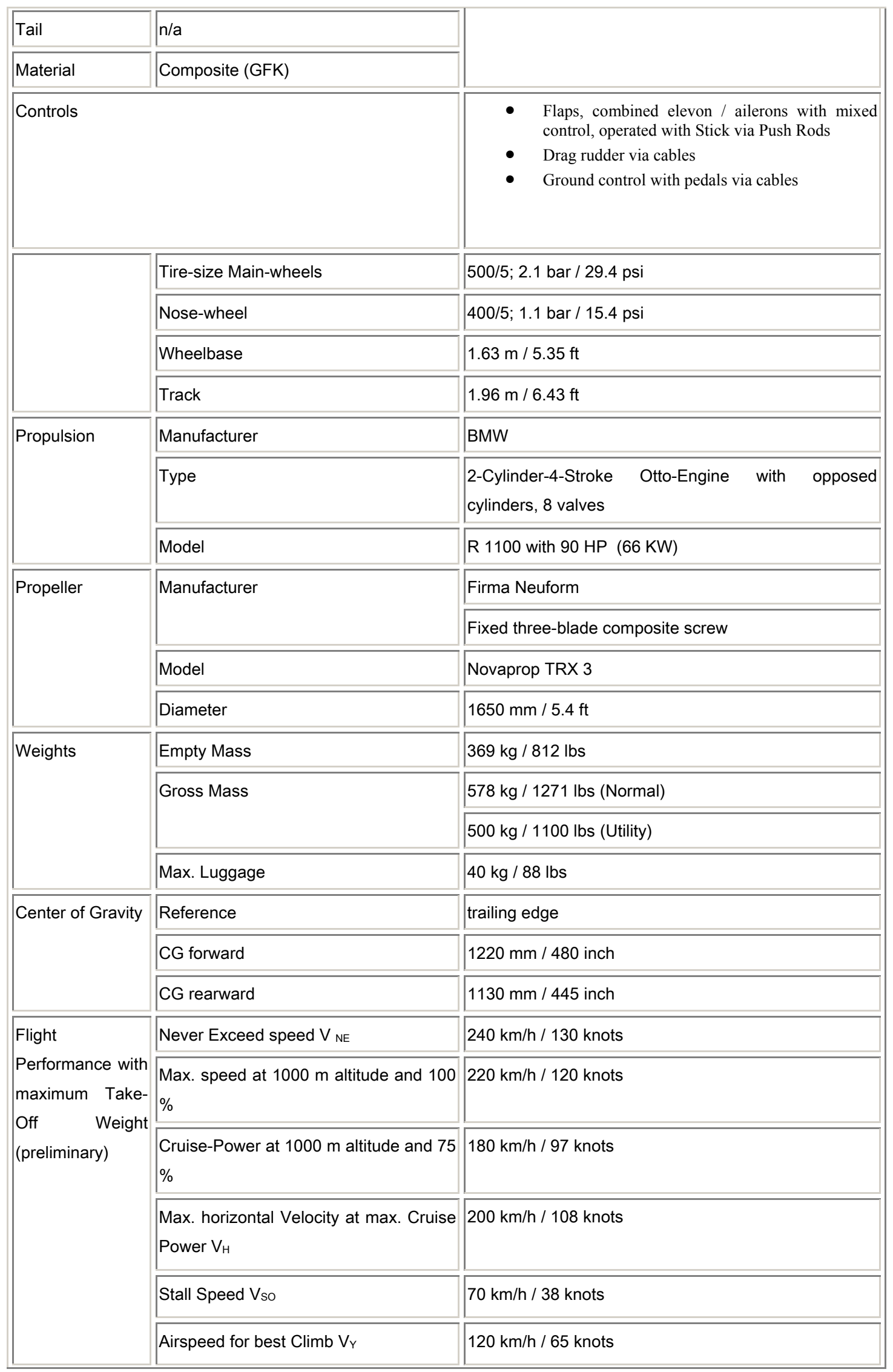




\begin{tabular}{|c|c|c|}
\hline & Climb Rate & $5 \mathrm{~m} / \mathrm{s} / 984 \mathrm{ft} / \mathrm{min}$ \\
\hline & Airspeed for steepest Climb $V_{x}$ & $110 \mathrm{~km} / \mathrm{h} / 60$ knots \\
\hline & Max. Altidude & $4000 \mathrm{~m} / 13000 \mathrm{ft}$ \\
\hline & Take-Off over $50 \mathrm{ft}$. obstacle & $1150 \mathrm{ft}$ \\
\hline & Landing from $15 \mathrm{~m}$ Altitude & $300 \mathrm{~m} / 984 \mathrm{ft}$ \\
\hline & Range & $1200 \mathrm{~km} / 648 \mathrm{NM}$ \\
\hline Design According & Classification & Normal / Utility \\
\hline & Max. Acceleration & $+3.8 ;-1.8 /+4.4 ;-2 \mathrm{~g}$ \\
\hline
\end{tabular}

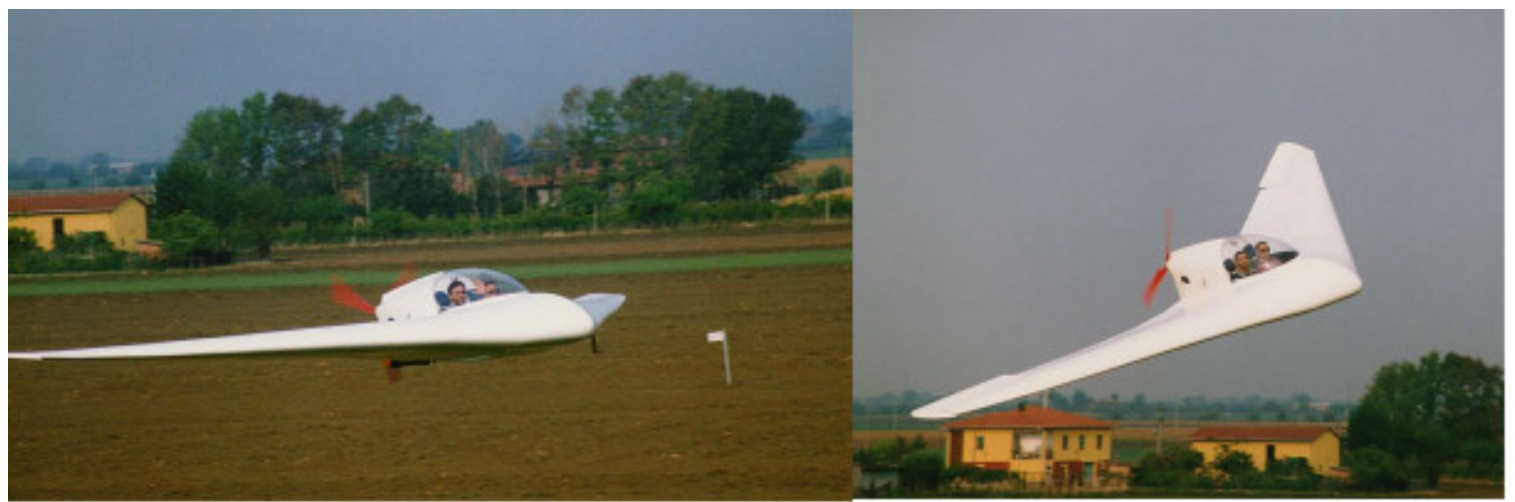

Figure 17- Horten-Panek PUL-10 in flight over Germany in 1987 (Bullard, 2003)

Reimar Horten developed the use of a bell shaped lift distribution on swept wing tailless aircraft as both cure for adverse yaw in the 1930's. These wings have wash-in initially, which increases the upwash toward the tip. Then Reimar displaced the wash-out toward the tips. This combination of airfoil shape, twist and sweep creates a resultant lift vector with a large forward component at the tip (Thrust). Using a bell shaped lift distribution, instead of an elliptical lift distribution (linear twist); would in theory minimize adverse yaw. This built in geometry only gives the desired control over a certain velocity and angle of attack range. In order to expand the range where adverse yaw was not a problem the Hortens used a full trailing edge, multiple elevon configuration to maintain the desired span. To date there have not been any formal control or aerodynamic studied of the Horten type tailless aircraft. (Bowers, 1999) Other designers have chosen to use winglets or drag flaps as a solution, but these also have additional drag associated with them.

In the late 1950's the Astrophysics Department of Mississippi State University (MSU) and Dezo George Falvy conducted a thorough aerodynamic study of the Horten IV tailless sailplane. (Figure 18 \& Figure 19) The initial studies done the unaltered Ho IV indicated that the performance of the aircraft was well below what early tests at DFS in Germany had shown, best gliding ratio of 0.37 . There are numerous reasons for this the 
primary one being the poor condition of the aircraft. The Ho IV was built during World War II, was hidden in a barn, captured by US troops, shipped to England and then shipped to the United States.

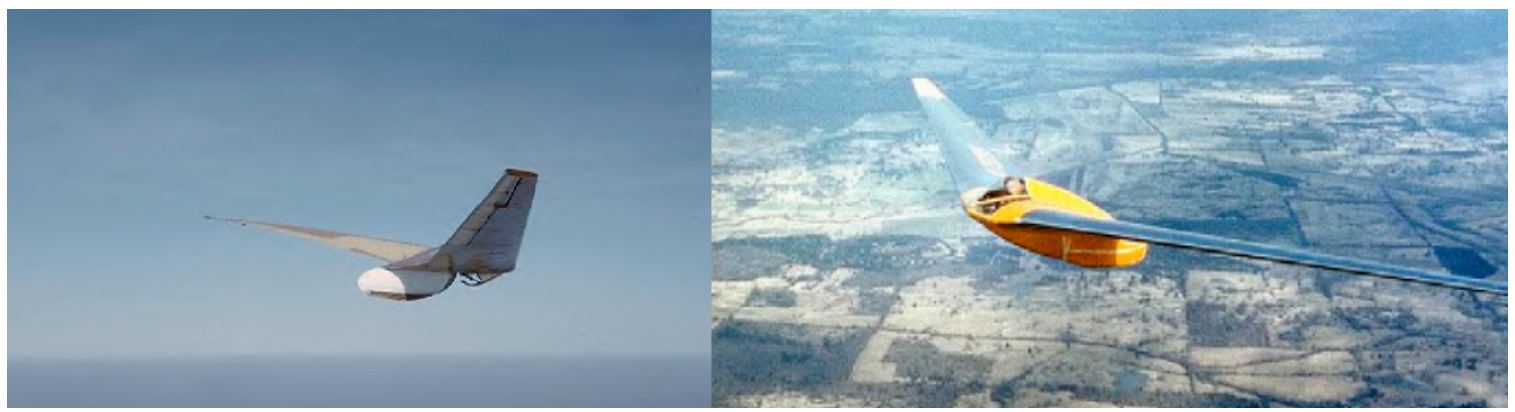

Figure 18 - The Horten IV during flight testing at Mississippi State University. The unaltered Ho IV with original landing skid can be seen on the left and the right shows the fairing built during testing in an attempt to improve the aircrafts performance. (Bullard, 2003)

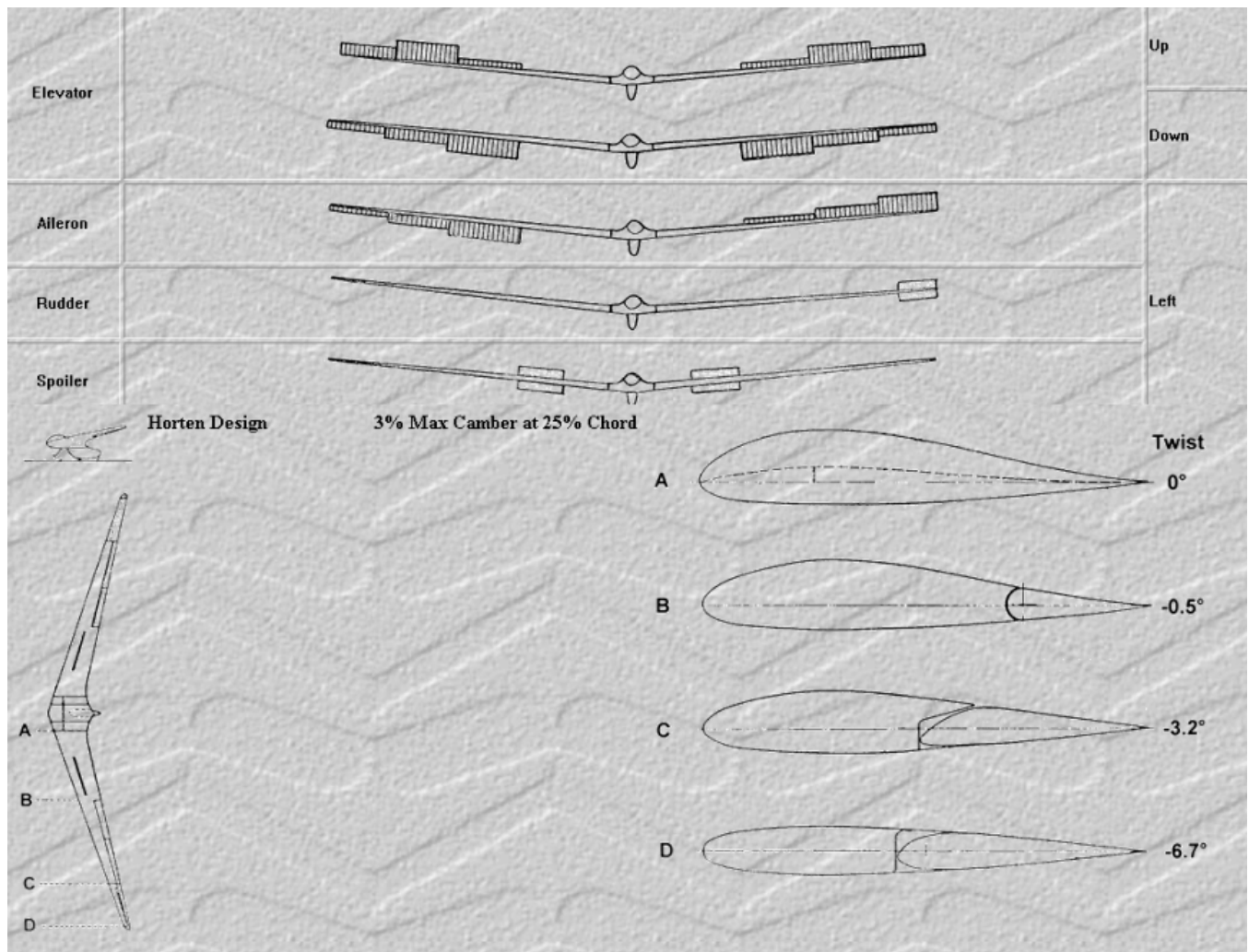

Figure 19 - Schematics of the Ho IV's controls, airfoil geometry and wash out geometry made by George-Falvy. Notice the Horten's use of the three pairs of elevons to achieve the equivalent of washout changes. (Bullard, 2003)

The wing surfaces had deteriorated or had been damaged and hastily repaired by the time of the MSU study. Reimar Horten also indicated that MSU did not achieve the same results as in Germany because they used the wrong Cg location, which required elevon trim for flight. MSU repaired the aircraft skins and attempted to modify the aircraft to 
improve it performance. One change was to add a fairing around the front landing skid, which may have actually degraded the aircrafts performance. The Landing skid fairing changed the aircrafts directional stability and further reduced the $\mathrm{Cg}$ range of the aircraft. Post alteration data indicated that the aircraft had a minimum drag coefficient $\left(\mathrm{C}_{\mathrm{Dmin}}\right)$ of 0.0125 and a maximum lift coefficient $\left(\mathrm{C}_{\mathrm{Lmax}}\right)$ of 1.125 . The major components of profile drag were protrusions from drive brakes, control surfaces and the Frise type elevons which all generated turbulent separation. Wool tuft photography showed that he major component of parasitic drag was the region around the cockpit particularly at low speed. The MSU study described the aircraft as having extremely good-natured stalling characteristics, good circling characteristics and excellent landing maneuverability. On the other hand the Ho IV had marginal directional stability and unusual control responses, which Reimar Horten attributed to the landing skid fairing and Cg location. This study concluded that the aircraft could be redesigned to achieve a theoretical Glide ratio of close to 50:1 and many of the poor handling characteristics could be remedied if the following changes could be made:

1) Reduction of profile drag through the use of laminar airfoils

2) An 8 to 10 percent reduction in induced drag, which is 30 percent of the aircrafts total drag if there was a way to change sweep in flight, a way to adjust the washout in flight and a way to adjust $\mathrm{Cg}$ in flight. (George-Falvy, 1997)

The MSU study was the first real analysis of the flight characteristics of a tailless aircraft and how they could be improved in the public domain. The recommendations they made are still valuable today. A new aircraft incorporating the lessons of this study was planned at MSU, but the death of Dr. August Raspet, of the Astrophysics department of MSU, ended these plans.

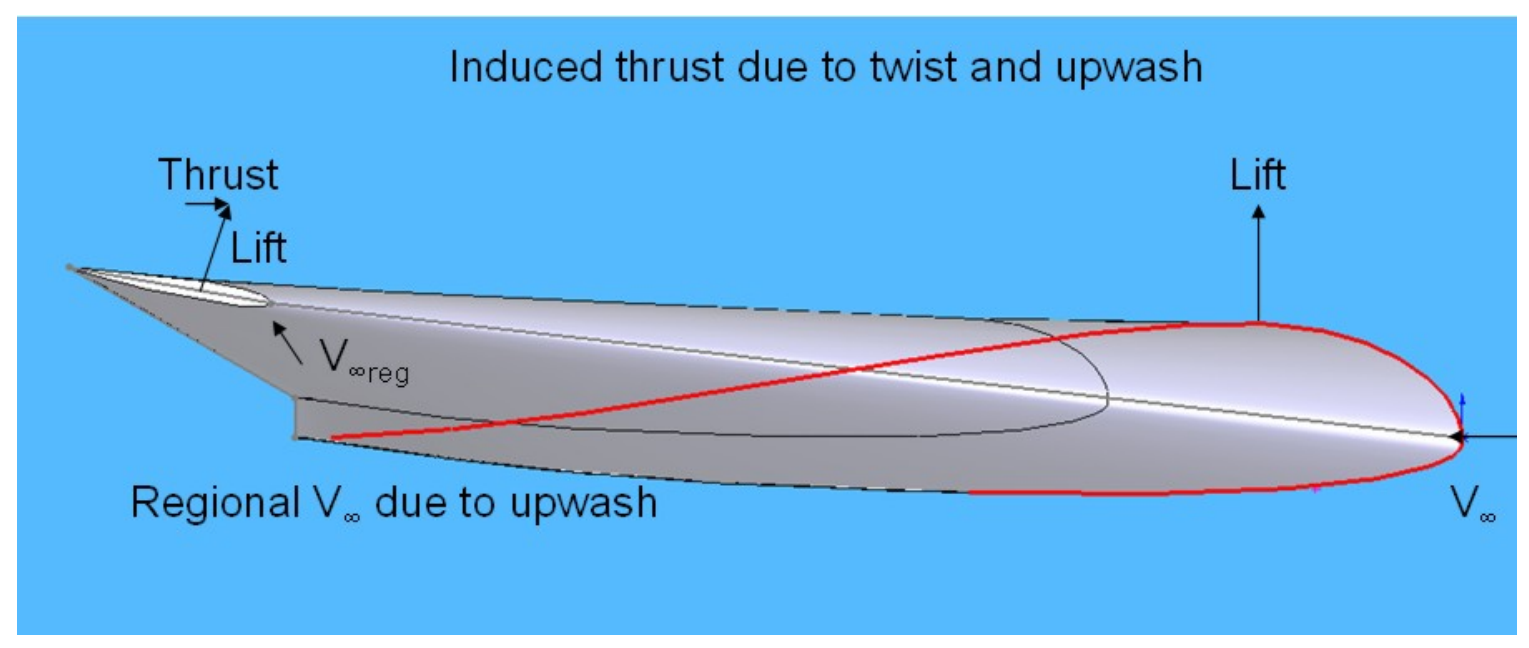

Figure 20 - Induced wingtip thrust due to wing twist and washout 
Building on the aerodynamic work that Dr. Raspet completed on Horten aircraft and his comparisons between Horten type aerodynamics and the soaring aerodynamics of a Black Buzzard, Dr. Philip Burger funded by the American Museum of Natural History is conducting research on bird flight which has some interesting parallels with Horten aircraft flight. Reimar Horten stated that with his aircraft designs he could get thrust from the wing tips at very high lift coefficients, (Figure 20) but there has been no formal data on this.

Dr. Burgers research has shown that where a Horten design gets thrust from sweep and upwash, a bird get thrust from the upwash produced by flapping. Studying video of an albatross taking off from water it can be seen that the wing flapping produces lots of thrust but very little lift. Using video and mathematical studies of the flow fields around a Pelican's wing in flight gives a new twist on conventional thinking on bird V-formation flight. Conventional think is that V-formations help to increase lift within the formation. Dr. Burgers research indicates that birds in these $\mathrm{V}$-formations gain aerodynamic thrust instead of lift. (Figure 21)

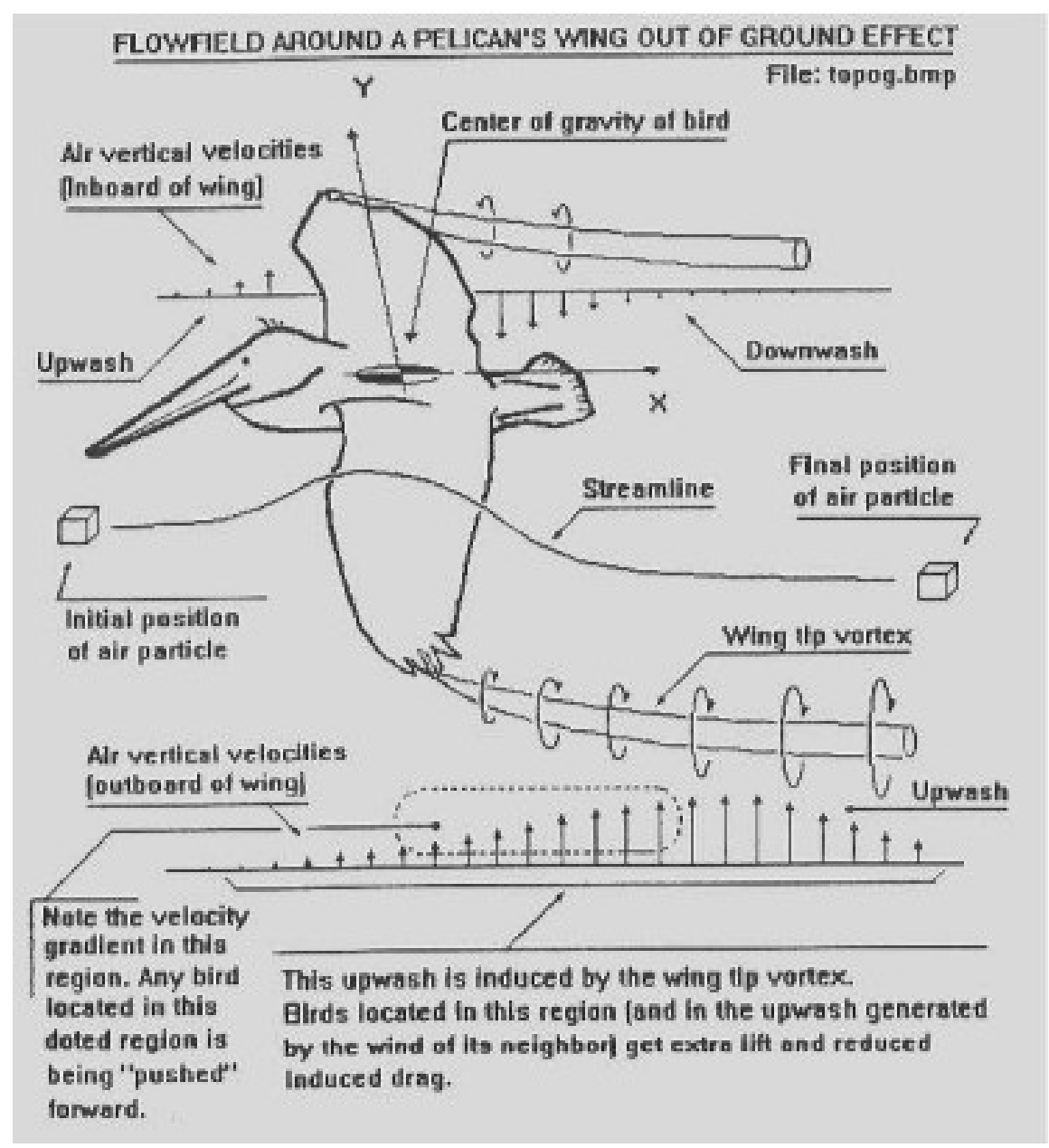

Figure 21 - Flow field and upwash around a pelican's wing 
Another similarity between the flow field around a Horten aircraft and a bird formation is sweep. Dr. Burger used CFD to study how the upwash from the lead bird in a formation effected the other birds in the formation. Then by various iterations at different sweeps, the minimum induced drag on the formation of birds was at a sweep angle of between 3040 degrees which is the same angle as most of the later Horten designs. (Burger, 2007)

\section{Recent Swept Wing Tailless Aircraft Developments}

There have been two truly innovative swept wing tailless sail planes developed since the Horten designs of the 30s, 40s, 50s, and 60s. These aircraft are the Arcus SB-13, Brightstar SWIFT. Both of these designs were developed to take advantage of advances in aerodynamics and materials through the use of laminar flow airfoils and lightweight composites.

The Arcus SB 13 was developed by the Akademischische Fliegergruppe Braunschweig between 1982 and 1988. (Figure 22) This is an academic organization of volunteers who had support from the German Ministry of Research. Their goal was to create a sailplane with the absolute minimum drag. They decided that a tailless configuration with a small fuselage and winglets would offer the best solution. They theorized that in this configuration they could create an aircraft with at least $10 \%$ better performance then the best sailplanes of the time. Unlike the Horten's they chose laminar airfoils with close to a zero pitching moment and used an elliptical lift distribution. Large winglets with rudder control surfaces were configured to minimize adverse yaw when maneuvering. The design work was fairing extensive on this aircraft involving computer aerodynamic and structural modeling and a $1 / 3$ scale remote control demonstrator. One major problem with a high aspect ratio aircraft such as this is designing a structure, which is light, but rigid enough to resist flutter. Scale structural models and computer modeling indicated that the aircraft would have a flutter mode at around $120 \mathrm{~km} / \mathrm{hr}$. This was cured with redesign of the spar, which pushed the flutter mode to above $310 \mathrm{~km} / \mathrm{hr}$. The spar was constructed of Carbon rovings and the wing skin was made of carbon fabric composites. 


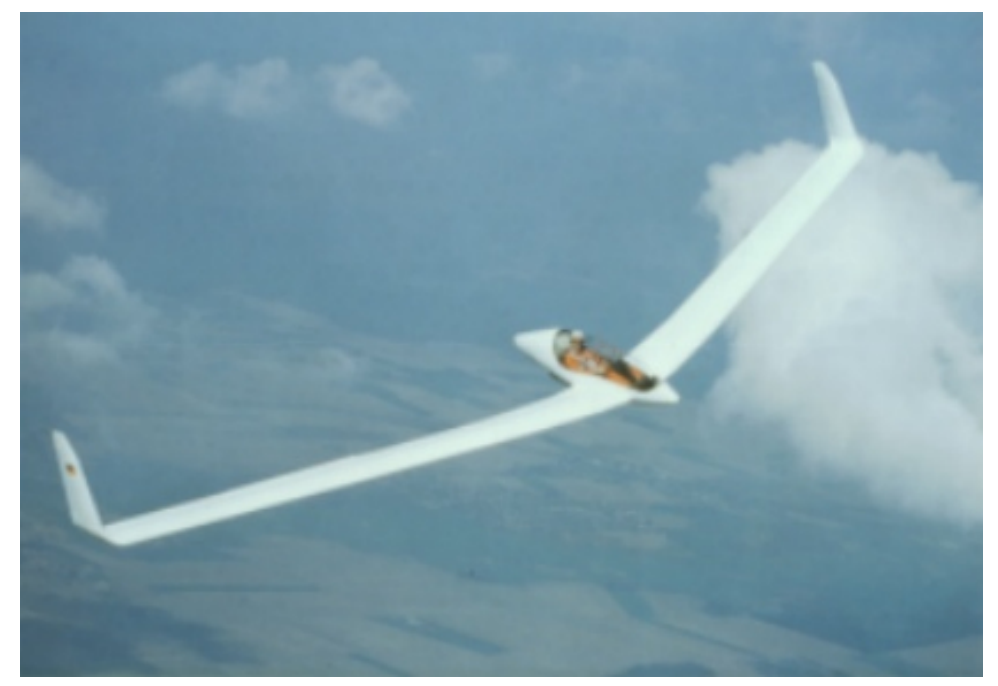

Figure 22 - Schematic of the SB 13 (Akaflieg Braunschweig, 2003)

The design of this aircraft is very well thought out, but the final performance was comparable with modern conventional gliders. A new design with the lessons of the SB 13 would be of value, but no more support was available. The final aircraft has an aspect ratio of 19.4, with a span of 49 feet and an empty weight of 649 lbs. (Akaflieg Braunschweig, 2003) When these numbers are compared with the Horten IV the achievements of the Horten brothers becomes apparent. The Horten's having no computer modeling and only wood and small amounts of magnesium alloy components created the Horten IV having an aspect ratio of 21.8, with a span of 66 feet and an empty weight of $551 \mathrm{lbs}$. Akaflieg Braunschweig did create the first tailless sailplane using modern composites.

Another group with similar goals as the SB 13 team was a team from Stanford University which created the Brightstar SWIFT ( Swept Wing Inboard Flap For Trim).(Figure 23) This aircraft was also an all-composite swept wing tailless aircraft with the distinction of being foot launched. The SWIFT design utilized a combination of sweep, taper and twist in order to make the use of conventional laminar flow non-reflexed airfoils. The majority of the large aerodynamic compromise made by using twist was negated by a large inboard flap which could be trimmed to change the effective twist. Tip mounted fixed winglets were used to reduce the maneuvering adverse yaw generated by the elevons. Extensive 3D aerodynamic and structural modeling along with a scale RC demonstrator were used to finalize the design. Flight testing began in 1989 in Northern California. Performance was satisfactory, but flight tests indicated that the control surfaces should be larger and the airfoil and winglet designs should be revised. A new aircraft with these improvements was then brought to the Owens valley in California were after a foot launch it flew 140 miles when it needed to be landed because of a malfunction in the pilot's oxygen system. The SWIFT is a remarkable tailless design with a lift to drag ratio of $25: 1$, a span of $38 \mathrm{ft}$ and an empty weight of $100 \mathrm{lbs}$. . (Kroo, 1991) 


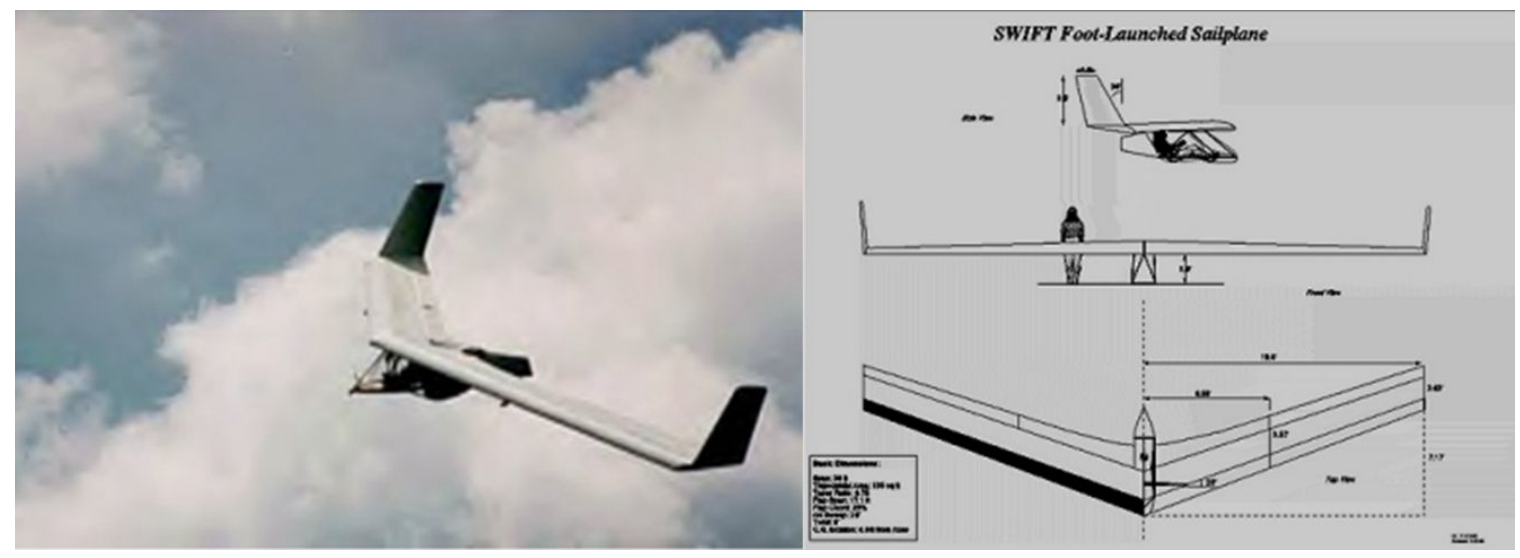

Figure 23- The SWIFT off the coast of Northern California and a 3-View Schematic. (Kroo, 1991)

There have been two recent additions to the category of large tailless aircraft, the Boeing Blended Wing Body (BWB) and the Northrop - Grumman B-2 bomber. Both aircraft take advantage of modern computer aided design and modern composites.

The Boeing BWB actually started at McDonnell Douglas before Boeing purchased the company. The original design project was another project to improve passenger-handling efficiency, much the same as today's Airbus 380 is striving to do. (Figure $24 \&$ Figure 25) Initially McDonnell Douglas and NASA Langley and Stanford University cooperated on the project. The original design study was for an aircraft to carry 800 passengers, 7000 $\mathrm{nm}$ at Mach .85. The blended wing configuration, championed by Boeing's Robert Leibeck, was expected to be $25 \%$ more fuel-efficient and $15 \%$ lighter then a comparable conventional airliner. This design used the turbofan engine to control the upper surfaces boundary layers by taking in air either through a perforated skin or long narrow intakes.

When the design was to the appropriate point the Stanford team developed two large RC flight demonstrators, one 6 foot and the other 17 feet in span. (Figure 26) These aircraft proved many design concepts. Later in the program as the design approached reality another flight demonstrator was created by the NASA Dryden and Langley teams. The current design utilized a large number of control surfaces to control the effective twist of the wings. There are tip mounted winglets with rudders and there is still some talk of the use of boundary layer controls although the engine intake boundary control idea was scrapped as being too complicated. There still are a number of design issues with the BWB design such as how to build a lightweight non-cylindrical pressurized structure effectively, how to deal with the human aspects like evacuation and roll sensation, how to deal with infrastructure limitations like span and runway loadings and finally the customer's perceptions of the aircraft. These problems along with budgetary problems have lead to most of the research being put on hold, but Boeing is still pushing for a cargo version of the aircraft. The benefits of this design are obvious and it is just a matter of time before Boeing or someone else develops a similar aircraft. 


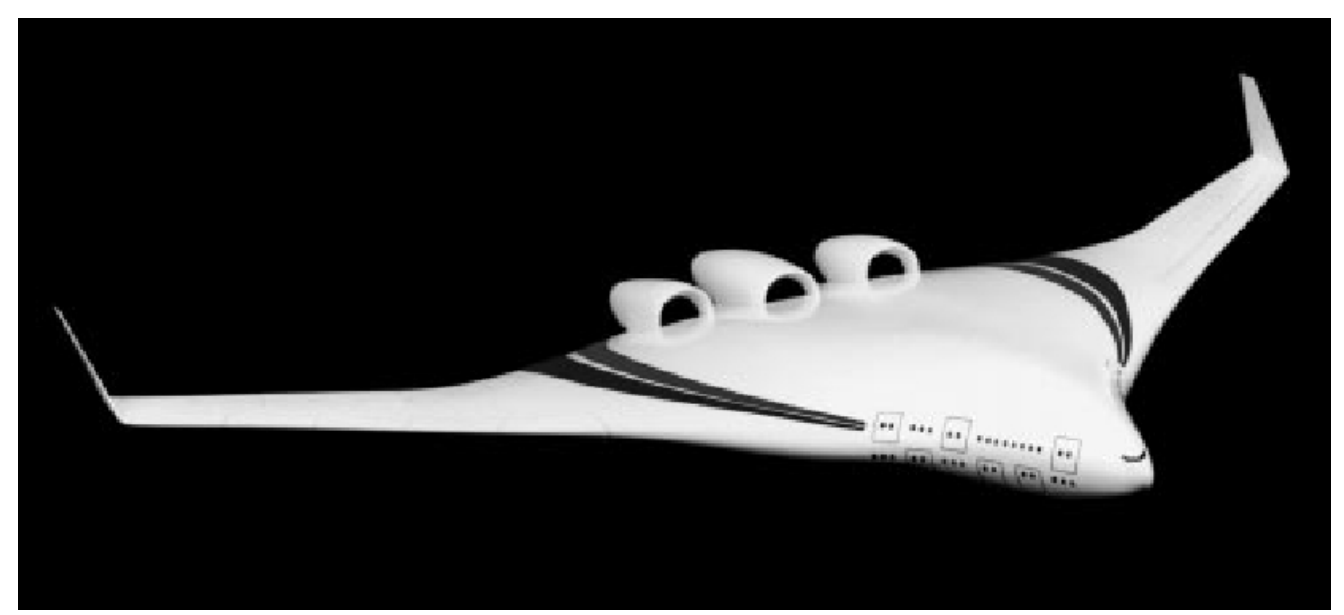

Figure 24 - Artists impression of the Boeing BWB (NASA, 1997)

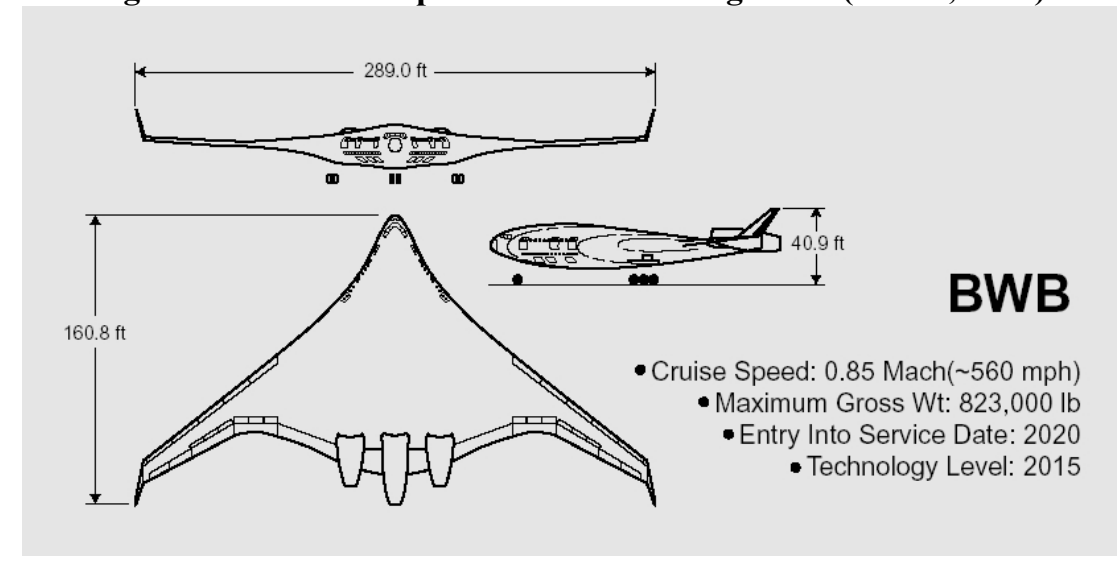

Figure 25 - Boeing Blended Wing Body schematic (NASA, 1997)

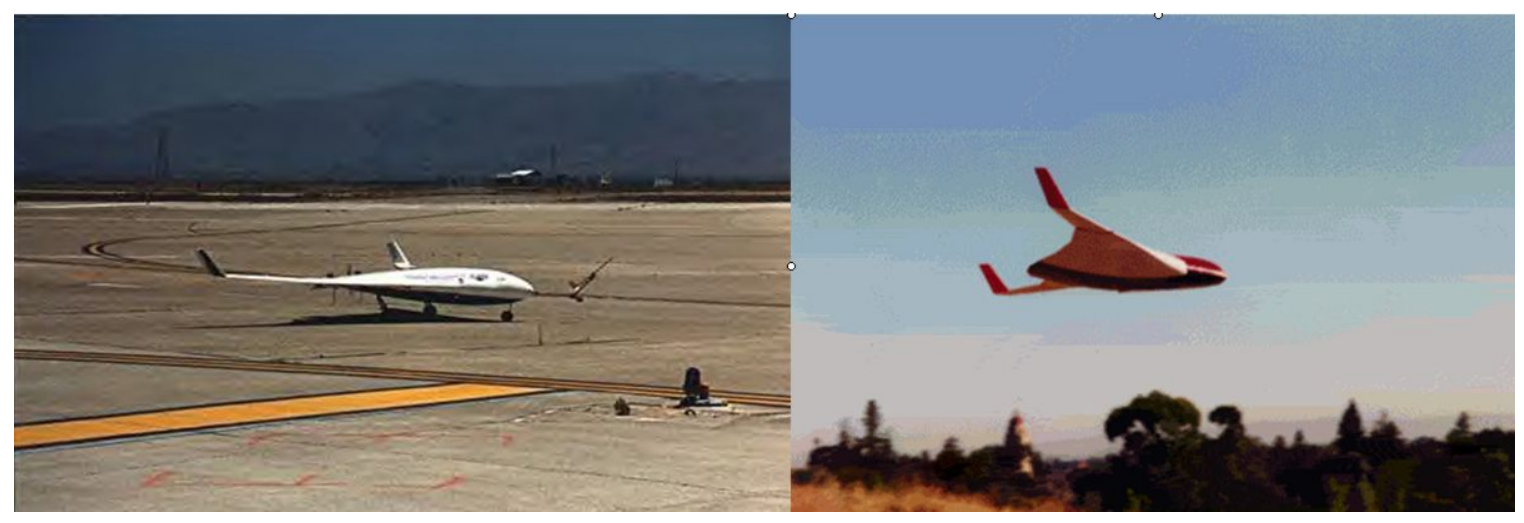

Figure 26.-.The 17 foot and 6 foot BWB flight demonstrators. (Kroo, 1995)

In the Spring of 2006 Boeing announced that it would compete with the Airbus A 380 with a 1000 passenger Blended Wing Body (BWB) called the 797 instead of the 747X. Boeing states that their current design will be 33\% more efficient than the A380 and will cruise at Mach 0.88. (Figure 27), (www.newstechspy.com, 2006) 


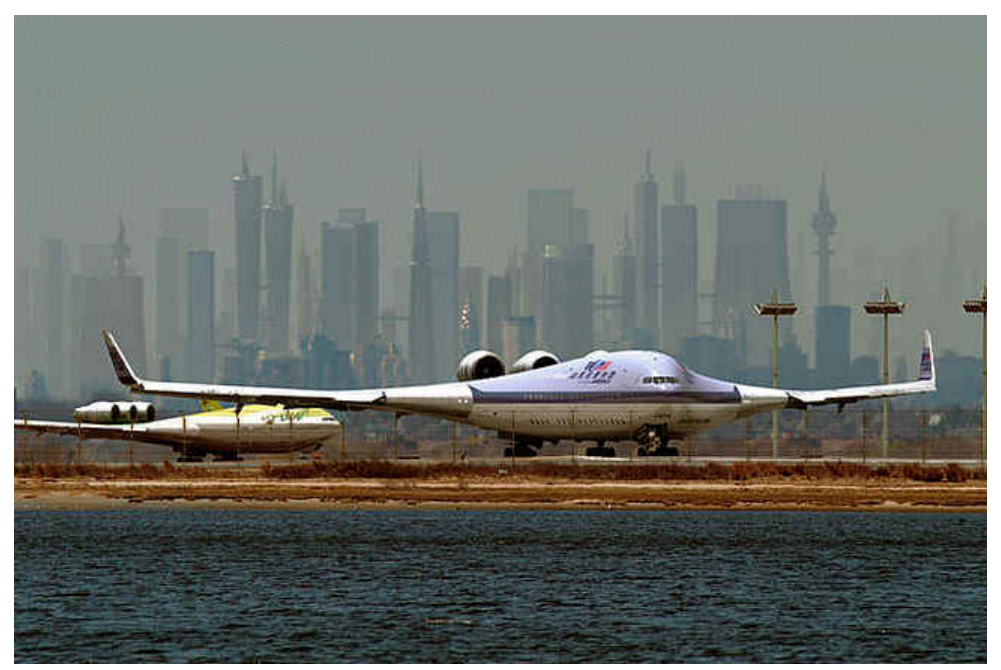

Figure 27 - Artists Impression of the proposed Boeing 797. (http://www.truthorfiction.com/rumors/b/b797.htm)

As of January 2007 a 21 foot span model of the Boeing BWB called the X-48B is continuing wind tunnel testing in NASA Langley's full scale wind tunnel as part of a joint Boeing, Air Force Research Lab and NASA project. Results are already indicating that the new aircraft has the potential to get up to $30 \%$ better fuel efficiency over conventional contemporary designs. This aircraft's digital flight controls command winglets, slats and numerous trailing devices to maintain efficient control over the aircraft. This wind tunnel model is also a flight demonstrator powered by 3 Williams International turbojets. (Barr, 2007)

Airbus also announced in the summer of 2006 that they also were continuing funding for their swept wing tailless BWB. The Airbus design is in its $5^{\text {th }}$ iteration and is currently undergoing aerodynamic and configuration studies. (Kresse, 2006)

The Northrop-Grumman B-2 bomber is the realization of what both Reimar Horten and Jack Northrop were trying to achieve. In the attempt to reduce the radar cross section Northrop designers came back to the pure flying wing with no vertical surfaces and smooth contours through out. This time the Northrop designers had the freedom to use all of the lessons of there founder Jack Northrop and the lessons of Reimar Horten. The designers studied the Horten aircraft in the National Air and Space Museum collection and had personal correspondence with Reimar Horten in Argentina. The result was a remarkable aircraft which uses efficient airfoils, computer aided design, modern composites, twist to help counter adverse yaw, split flap drag rudders and digital flight controls. The B-2 combined all of the tailless design ideas into one aircraft. With a wing span of $172 \mathrm{ft}$ and it's 4 efficient GE F118 turbofans the B-2 can fly up to $6000 \mathrm{~nm}$ unrefueled. (Figure 28) Over 20 of these aircraft have been produced and are serving with the US Air Force. They have been used in combat and have a flawless record. This aircraft is the zenith of what can be achieved with tailless aircraft and conventional controls. 


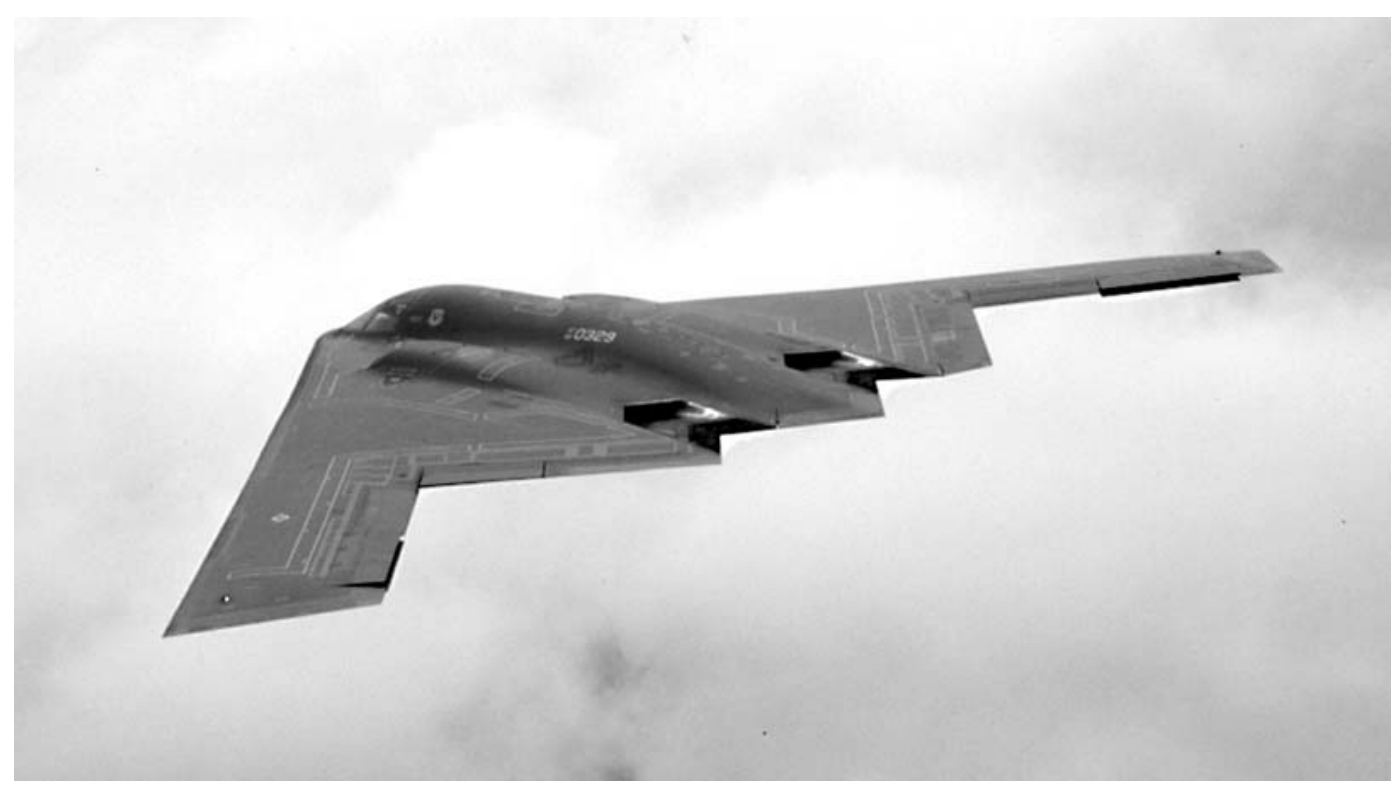

Figure 28- The graceful B-2 bomber in flight (Sweetman, 1999)

\section{Karl Nickel}

Dr. Karl Nickel was one of the principles in Horten Flugzeug Bau during World War II and then went with Reimar to Argentina. He was involved in most of the Horten designs through the 50's. He went on to continue tailless aircraft research as a Professor in Germany. In 1981 Dr. Karl Nickel designed a tailless swept wing aircraft which utilized a flexible fabric skin over a tubular frame, (Figure 29). This aircraft was called the Falter and was primarily controlled by changing the twist of the outboard wing sections. Flight testing of this aircraft uncovered no adverse yaw with the ability to enter controlled 5 to 10 degree side slips. In 1994 he published the first and only textbook on the design of tailless aircraft. In his book "Tailless Aircraft" he comes to the conclusion, based on his years of experience as a designer and a test pilot, that the use of the "bell shaped lift distribution" should not be used for tailless aircraft for the following reasons. 


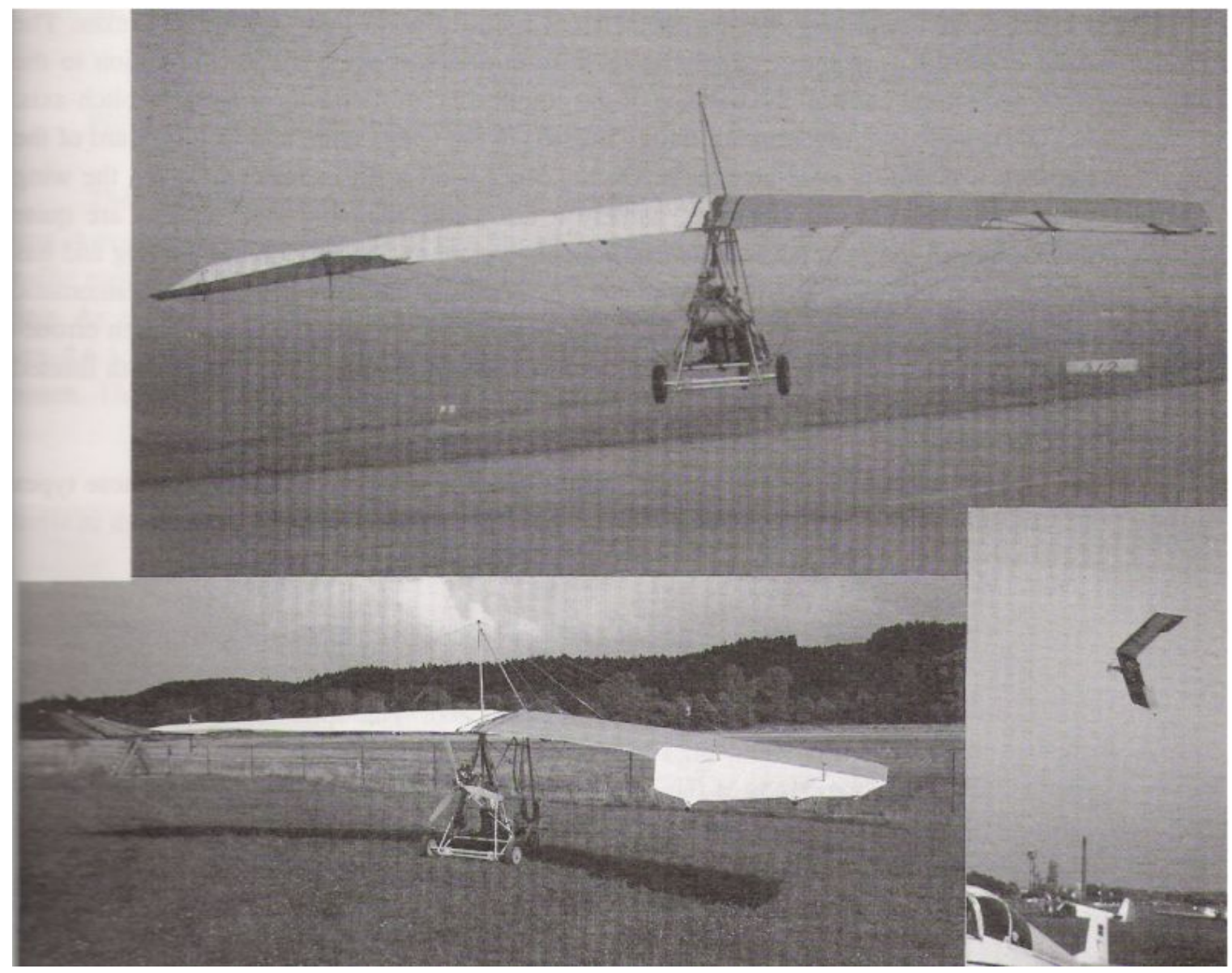

Figure 29.-.The Nickel Falter utilizing flexible skins and variable washout for primary control of a swept wing tailless aircraft (Nickel, 2005).

1) The bell shaped lift distribution was used to negate the induced drag which causes adverse yaw. Pilots who flew Horten aircraft all indicated that adverse yaw was still a problem. Therefore there is no reason to use it.

2) There are severe disadvantages to using the bell shaped lift distribution, mainly the additional induced drag. The bell shaped lift distribution is to cure a maneuvering problem, but the additional drag is carried during all flight regimes

\section{Lockheed Martin}

In July 2006 Lockheed Martin unveiled a secret stealthy UAV called the Polecat. (Figure 30) This aircraft has a 90 foot span and based on the work of the Hortens. The Polecat also allegedly either currently or in the future will incorporate morphing technologies such as adaptive washout and a morphing extendable tail. The current mission for the Polecat is high altitude reconnaissance. (Defencetech.org.org, 2006) 


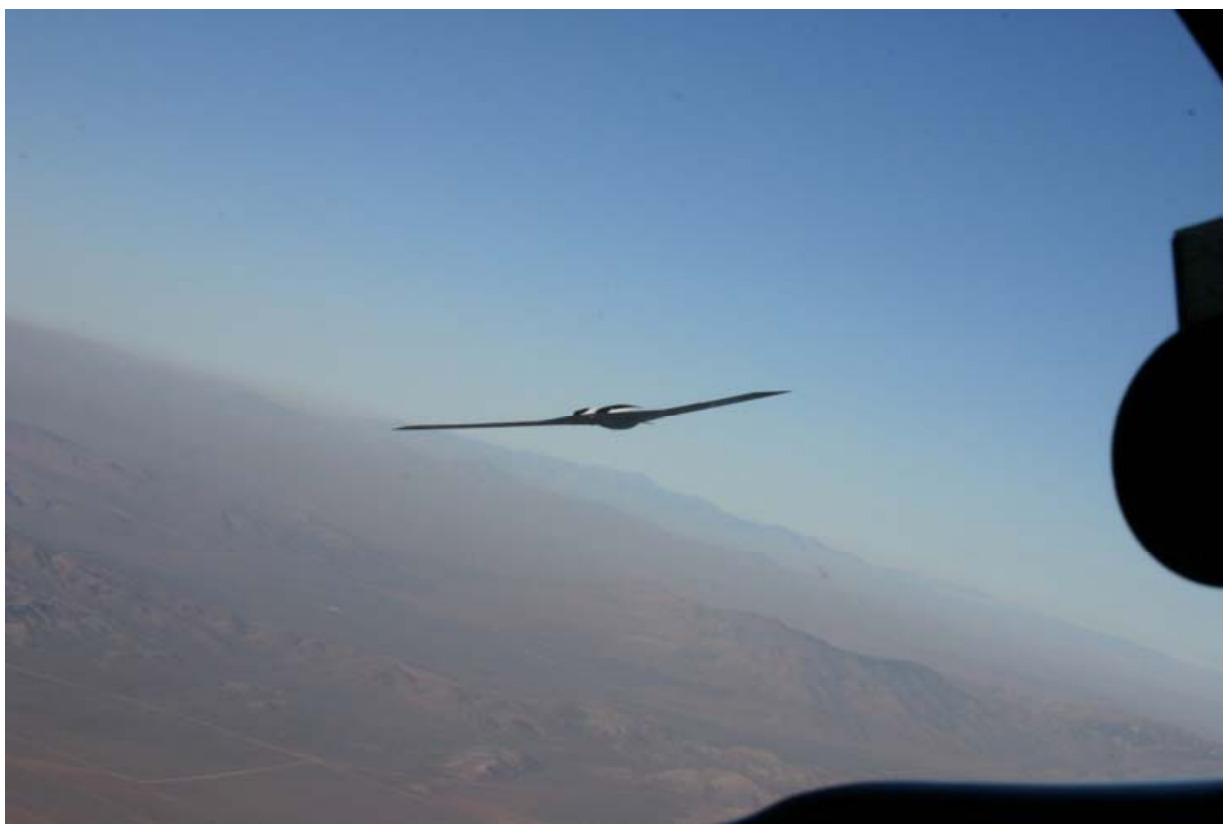

Figure 30 - Lockheed Polecat high altitude UAV which will in the future use wing morphing for control.(http://www.lockheedmartin.com/wms/findPage.dsp $=$ fec\&ci $=17787 \&$ rsbci $=0 \& \mathrm{fti}=1$ ) 


\section{Chapter 2 Wing Morphing}

\section{Early Aircraft Morphing Developments}

Advances in lightweight materials, actuator technology and modern aerodynamics have all come together in attempt to imitate the agility seen in bird flight. This new technology is called wing morphing.

The ideas are as old as manned flight, but the technologies make it more feasible today. One of the control mechanisms the Wright Brothers used in their original gliders and the Wright flyer was wing warping, which the earliest documented wing morphing. (Figure 31) In 1899 the Wright Brothers constructed a glider made of French sateen fabric, which covered a wooden frame. The fabric and the wood structure were sewn together this created a strong lightweight, but flexible structure. They oriented the fabric weave at 45 degrees to the aircraft flight direction, which allowed the structure to warp properly when pulled by actuation cables. This 1899 glider was build solely to test the idea of wing warping. This biplane configuration with steel wire bracing with wing warping was supposedly inspired by observing how a cardboard bicycle tube box could be warped yet still be ridged when returned to its normal configuration.

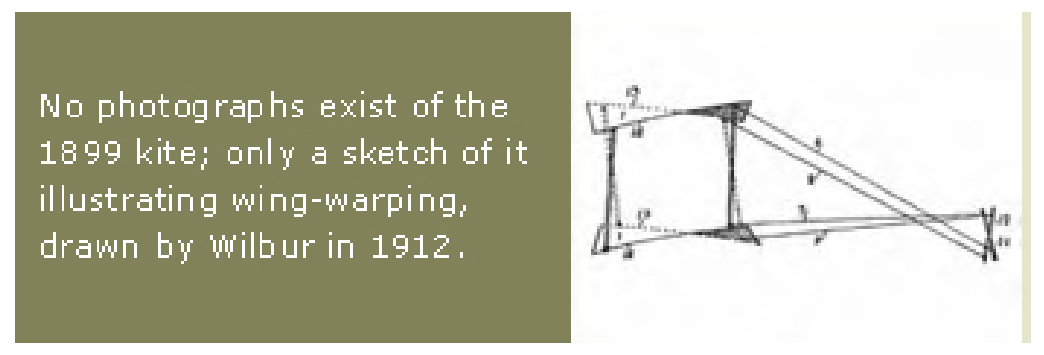

Figure 31 - The Wright Brother's 1899 wing warping design (NASM, 2004)

\section{NASA Mission Adaptive Wing (MAW)}

The field of wing morphing was fairly quiet until the 1980's when a joint US Air Force and NASA program called the Advanced Fighter Technology Integration (AFTI) began. One aspect of this research focused on what was called a Mission Adaptive Wing (MAW). A NASA F-111A's wing was replaced with a MAW wing built by Boeing Aircraft. This wing had an internal mechanism to flex the outer wing skin and produce a high camber section for subsonic speeds, a supercritical section for transonic speeds, and symmetrical section for supersonic speeds. The use of flexible wing skins to produce a smooth upper surface brought this wing a little closer in concept to that of a bird. A digital flight control system provided automatic changes to the wing geometry. The system had four automatic control modes: (1) Maneuver Camber Control - adjusting camber shape for peak aerodynamic efficiency; (2) Cruise Camber Control - for 
maximum speed at any altitude and power setting; (3) Maneuver Load Control providing the highest possible aircraft load factor (4) Maneuver Enhancement Alleviation - in part attempting to reduce the effects of gusts on airplane ride. The AFTI/F-111 MAW system had 59 flights from 1985 through 1988. (Figure 32) The flight test data showed a drag reduction of around 7 percent at the wing design cruise point to over 20 percent at an off-design condition. The four automatic modes were tested in flight with satisfactory results. (Curry, 2004)

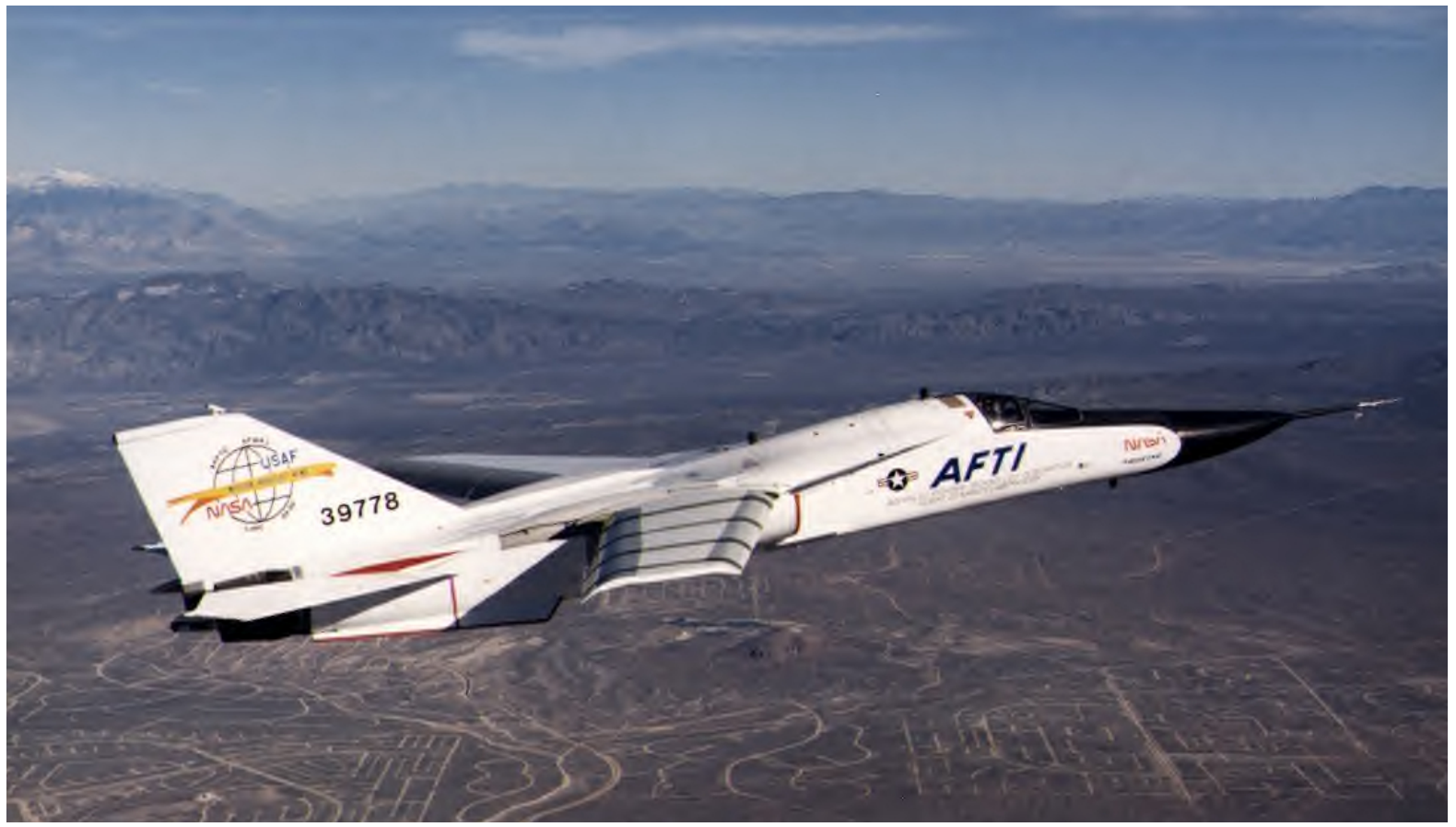

Figure 32 - The USAF-NASA F-111A MAW in flight at Dryden Flight Research Center (Curry, 2004)

3. NASA Active Aero-elastic Wing (AAW)

In 1996, the NASA Active Aeroelastic Wing (AAW) project began. (Figure 33) This is a joint project between NASA's Dryden Flight Research Center, Edwards, Calif., the U.S. Air Force Research Laboratory (AFRL) and Boeing Phantom Works, which is researching an adaptation of the Wright Brothers wing-warping approach to aircraft flight controls using modern composites and actuators. 

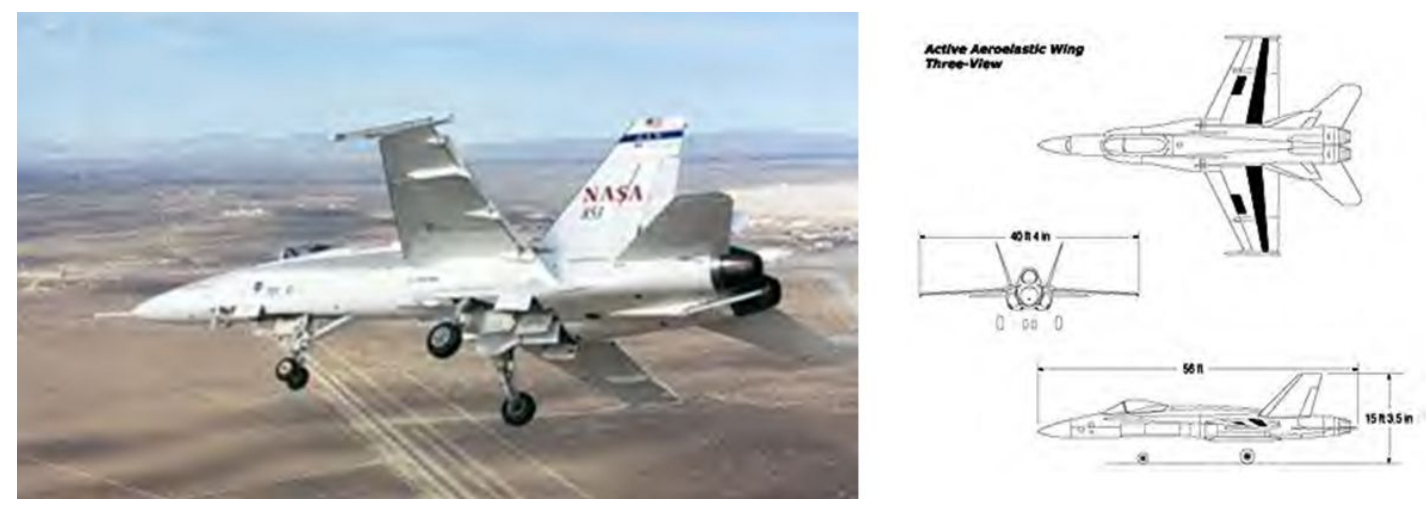

Figure 33 - F-18 AAW in flight and F-18 AAW schematic. (Curry, 2005)

The main focus of AAW research is to develop and validate the concept of morphing (wing warping) for roll control in modern aircraft. The AAW research demonstrator is a modified F/A-18A. Several of the existing wing skin panels along the rear section of the wing just ahead of the trailing-edge flaps and ailerons have been replaced with thinner, more flexible skin panels and structure. The F/A-18's leading-edge flap has been divided into separate inboard and outboard segments, and additional actuators have been added to operate the outboard leading-edge flaps separately from the inboard leading-edge surfaces. By using the outboard leading edge flap and the aileron to twist the wing, the aerodynamic force on the twisted wing will provide the roll forces desired. (Curry, 2005) The F-18 AAW is currently completing a two-phase flight test program with satisfactory results.

\section{NASA Biologically Inspired Aerodynamic Geometries}

A group of researchers at NASA Langley Research Center is investigating the directions in which wing geometry research should be headed. This research is in biologically inspired hydrodynamic structures and the control of wing morphing. Various structures in nature such as a seagull's wing, a hammerhead sharks head shape, a sharks fins and a humming birds wing in flight. We now have the technology to duplicate simple versions of these structures and the capability to begin to understand the complexity of structures and their control in nature. The Langley group is looking at these geometries, dynamic and static to learn how flight works in nature and then attempting to duplicate it with morphing aircraft. One new geometry called the hyper-elliptic wing inspired from nature has shown that gains of $15 \%$ in lift over drag are possible and this just scratches the surface on what we can learn. (Figure 34) This research is not just looking at biologically inspired geometries but is looking at how we can duplicate them in air vehicles; it is multidisciplinary, studying hydrodynamics, structure, controls, and actuation technologies. (Lazos, 2002) 


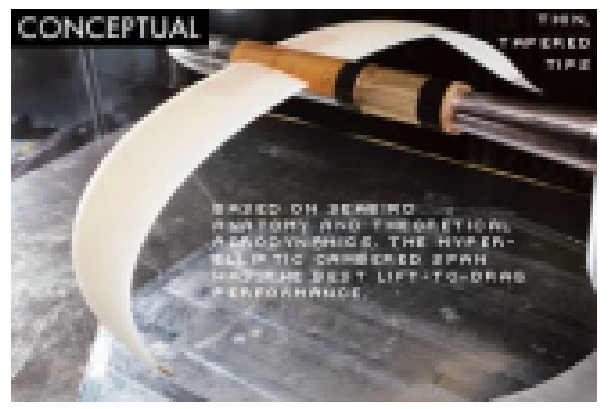

Figure 34 - Hyper-elliptic wing wind tunnel model (Lazos, 2002)

Now that technology makes wing morphing possible there are numerous research projects exploring what possibilities exist. A joint project between the Air Force Research Labs, Perdue University and Wright State University created a document exploring many of the possibilities on what a customer such as the Air Force could ask for in a morphing aircraft. A joint Air Force, Virginia Tech project looked at computer modeling wing shape changes and what actuation forces would be needed to duplicate the control found in today's conventional wings.

\section{DARPA / Lockheed MAS}

A joint Darpa and Lockheed Martin called the MAS project is exploring large area changes the wing of a tailless UAV demonstrator. (Figure 35, Figure 36 \& Figure 37) This project is using new piezoelectric actuators to fold and unfold a UAVs wings in flight. Twist near the wing tips, thrust vectoring paddles and a small areas of the trailing edge and leading edge near the center of the aircraft can also morph for elevon type control and pitch trim control. The actuators for this program come from a joint Darpa, Penn State project called the Compact Hybrid Actuators Program (CHAP). These actuators are very efficient, very volumetrically small. They can sit in small spaces in the wing and bring your actuation power to the place in the mechanism where it's needed. If you had one big actuator you'd have to do force transmission from a central location and that adds a lot of material and weight. (Koopman, 2004) Currently the wing folding will change the flight regime of the aircraft, but in the future it will be used to control the airplane. The UAV will operate bird-like control by pulling one wing in and rolling the other one, like a sea gull. This arrangement may save weight by not needing the usual control surfaces. (Marks, 2004) 

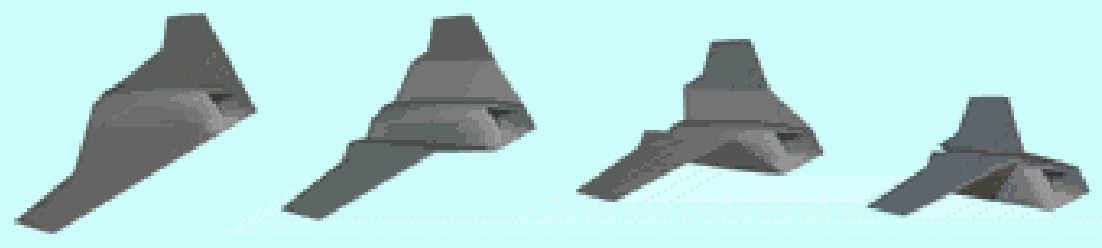

Figure 35 - The Lockheed Martin Tailless morphing demonstrator (Marks, 2004)

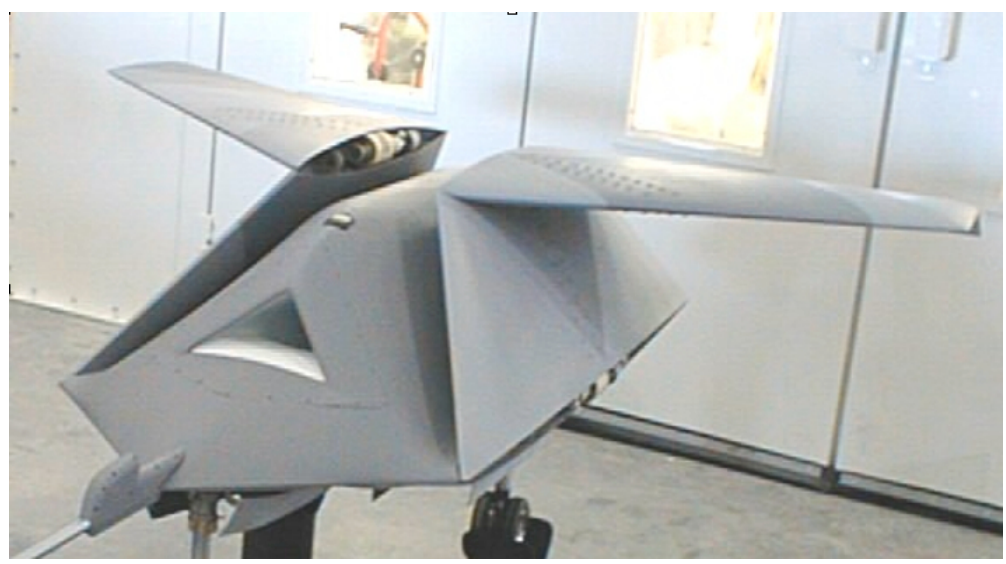

Figure 36 - The first Lockheed Martin (MAS Project) Tailless morphing (Folding wing) demonstrator (Koopman, 2004)

\section{PENSTIAIE Smart Wing Fold Mechanisms}

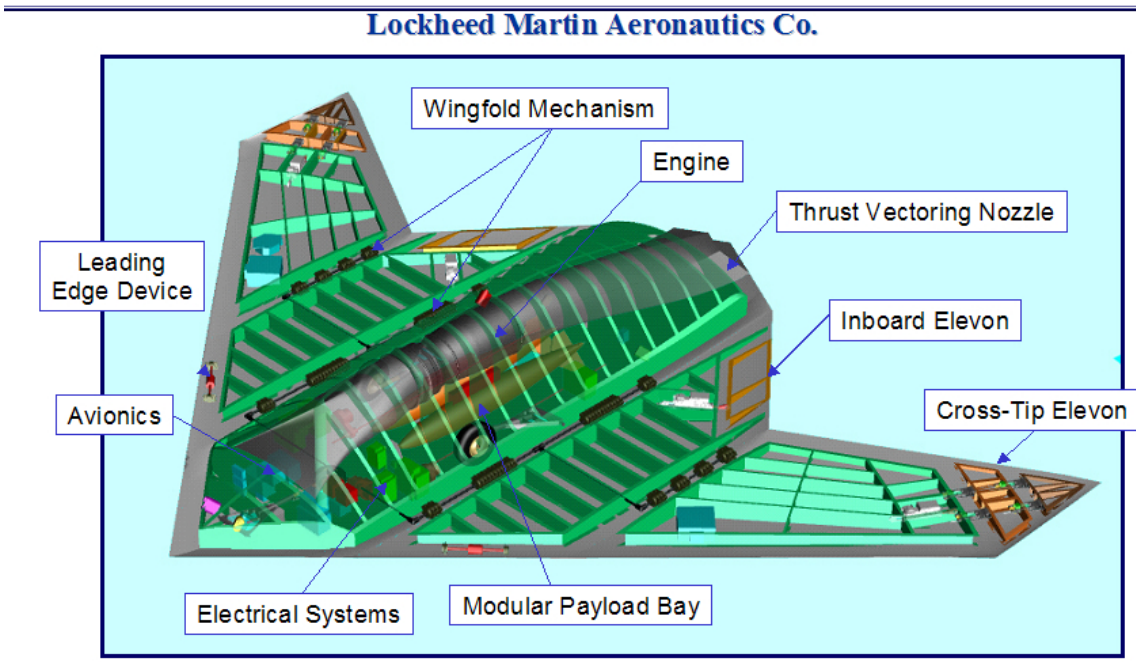

Special MAS Program Requirement - Smart Material Actuators

Center for Acoustics and Vibrations

Figure 37- Cutaway of Lockheed Martin MAS UAV (Koopman, 2004) 


\section{University of Florida}

In 2003 Helen Garcia and her colleagues at the University of Florida explored the use of active wing morphing for roll control of a 24" UAV and a 12" UAV. (Figure 38 \& Figure 39) Both aircraft were designed with the conventional wing followed by a tail with a rudder and elevator. The wing on the 24" aircraft had a torque rod running through the leading edge of the wing. Riblets of carbon roving trailing behind the torque rod. Between the riblets was a latex membrane. The 12" UAV used similar construction, but lacked the torques rod.

Control of the 24 " UAV was achieved by rotating the torque rod from a fuselage mounted servo. Three quarters down the wing the torque rod made a bend to then become parallel with the flight direction. When the rod was rotated imparted a twist to the wing. This twist created a roll and yaw moments.

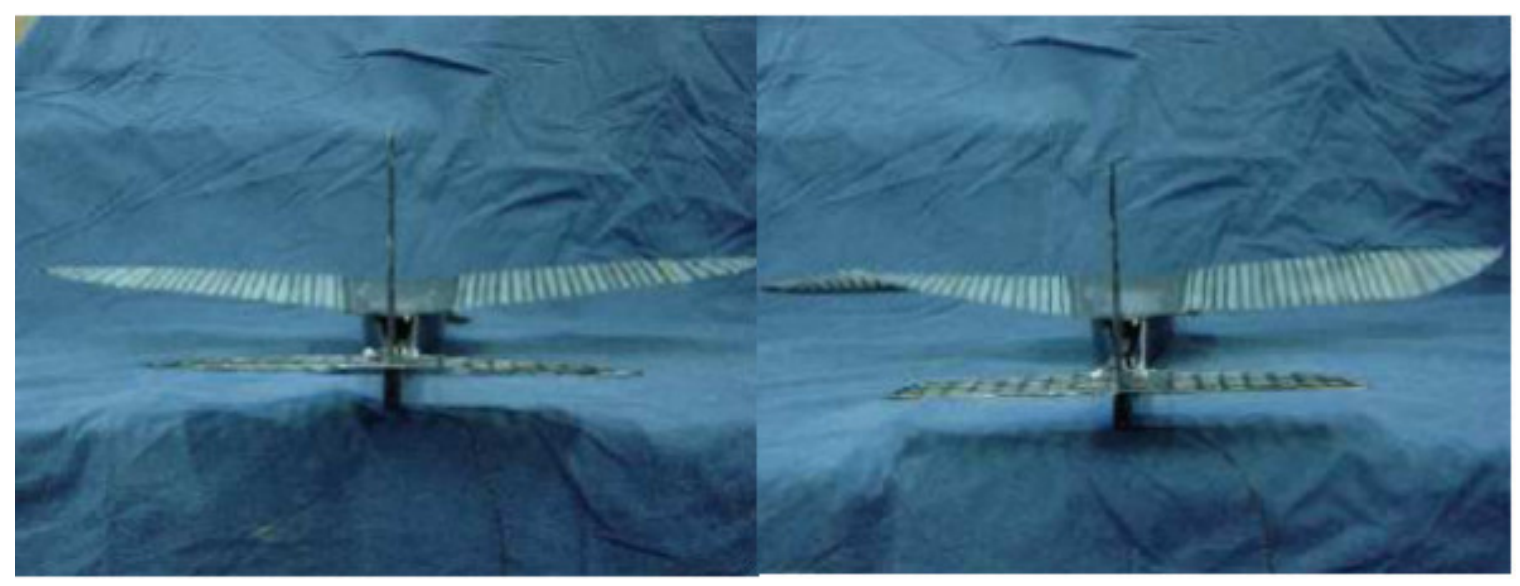

Figure 38- University of Florida 24" "twisting” morphing demonstrator. ( Garcia, 2003)

The 12" aircraft had a kevlar string which ran diagonal from the wing tip to the fuselage. A servo in the fuselage could pull the cable or let it out which both twisted and curled the wing tip to generate control forces. 


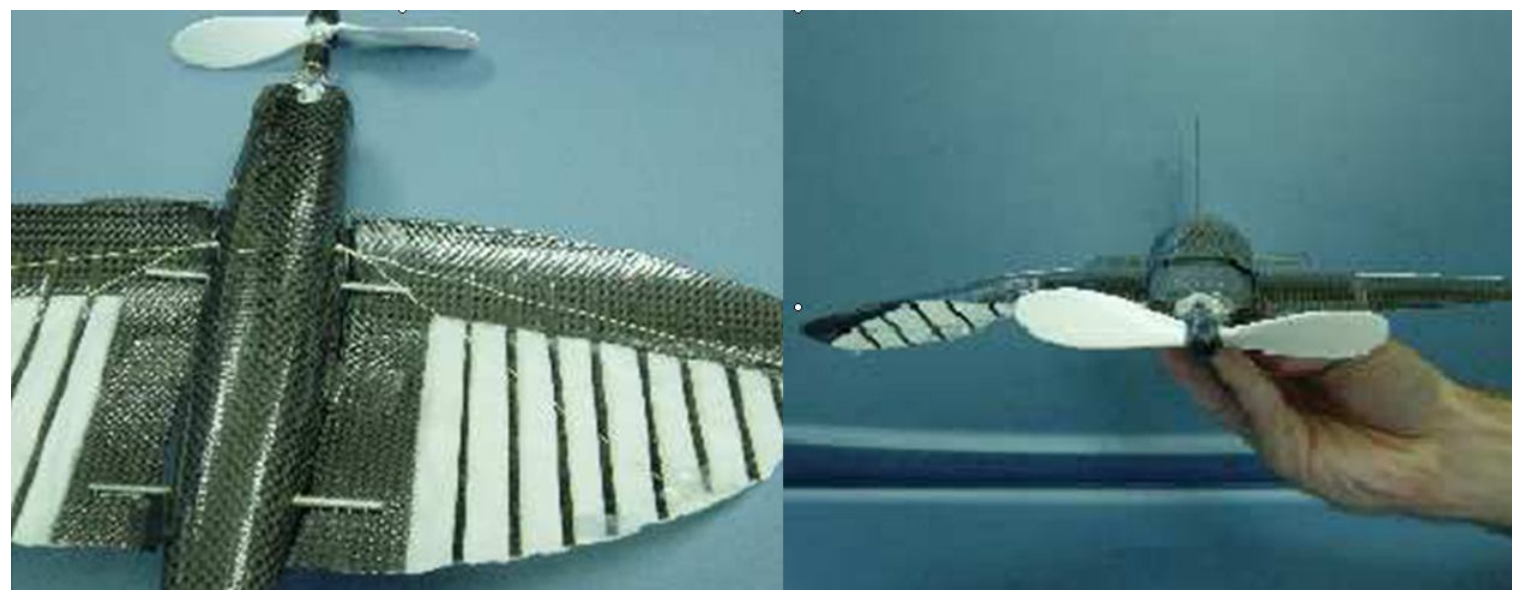

Figure 39 - University of Florida 12" "curl" morphing demonstrator. (Garcia, 2003)

Both morphing strategies twisting and curling proved well suited for roll control. Roll and yaw rates of close to 70 degrees per second and 20 degrees per second. Both aircraft had good flight characteristics and proved that significant roll rate could be generated with the twisting and curling morphing strategies. (Garcia, 2003)

\section{Current Morphing Component Technology}

\section{Electric Actuators}

Achieving the fluid transformation from one wing shape to another as a bird does requires new actuation technologies. Most of the cables and hydraulics common in aircraft today need to be replaced by actuators, which are actually part of the aircraft structure. Morphing aircraft structures emulate structures found in marine and airborne animals where the muscles, bones, skin and feathers combine to form a biological control surface. Standard hydraulic or servomotor actuators a can be used to flex or morph a composite aircraft structure. Pneumatic actuation can actually form a rigid structure and actuate changes in shape. There are also actuators, which act more like muscles in a living organism and these are piezo-actuator or metallic-actuators. These new type of actuators can be embedded in an aircraft skin or structure and when a voltage is applied can initiate a shape change.

\section{Servo-actuation}

Electric servo have been in use for over 50 years there power and efficiency have dramatically improved in recent years with the current electronics revolution. Many current servos are digitally controlled and can be programmed to modify their 
performance. Servos range in weight and power from $0.10 \mathrm{oz}(3 \mathrm{~g})$ and $6 \mathrm{oz}-$ in $(500 \mathrm{~g}-\mathrm{cm})$ torque to many pounds and thousands of pounds force.

\section{Pneumatic-actuation}

Recently the need has arisen for small UAV platforms to have deployable, controllable wings that may also change to fit a variety of flight regimes. In 2004 a group from ILC Dover wrote a paper which discussed efforts in reshaping, or morphing, inflatable wings to provide roll control through wing warping, i.e. actuation of the aft end of the wing to achieve changes in section camber. Several approaches have been developed that lend themselves to camber control via locally altering the geometry of the wing. Apart from use as a stand-alone aerodynamic surface on a small UAV, the inflatable assemblies can also be used as an aspect ratio increasing device on a larger aircraft to enable a more radical change in wing configuration. This approach serves to improve system efficiencies across changing flight regimes, allowing transitions from high speed target approach to low speed loitering. Shape changes in wing can be created through the use of pneumatic chambers under a flexible skin. (Cadogan, 2004)

\section{Piezoelectric and Ceramic Film Actuators}

NASA Langley Research Center has also been conducting research into what materials can be used to create self adaptive airframe system and how top use them. This research has focused on two main classes of materials: high performance continuous-fiber reinforces polymer matrix composites and piezoelectric films from high temperature polyimides. Piezoelectric devices have been identified as a promising actuator technology for the implementation of active boundary layer control, high bandwidth noise suppression and aeroservoelastic tailoring. However, many potential aerospace applications require displacement performance larger than what is achievable in conventional piezoelectrics. NASA Langley has developed two high-displacement piezoelectric actuator technologies, RAINBOW (Reduced And INternally-Biased Oxide Wafer) and THUNDER (THin layer composite UNimorph ferroelectric DrivER and sensor) to meet these requirements. These devices are unimorph-type actuators, which consist of a piezoelectric ceramic layer bonded to one or more non-piezoelectric secondary layers. Because of the use of elevated temperatures during processing, internal stresses are created in the structures that significantly enhance displacement through the thickness of the devices. Currently, the processing and characterization of these highdisplacement actuators are under investigation. One recent characterization study involved the effects of electric field, load and frequency on the displacement properties of rectangular THUNDER devices. Results showed that individual actuators were capable of free displacements in excess of $3 \mathrm{~mm}$ when tested at $\pm 9 \mathrm{kV} / \mathrm{cm}$. (Figure 40) Increasing device stiffness through metal selection and thickness resulted in improved load bearing performance at the expense of displacement, allowing devices to be designed with a range of performance capabilities. 

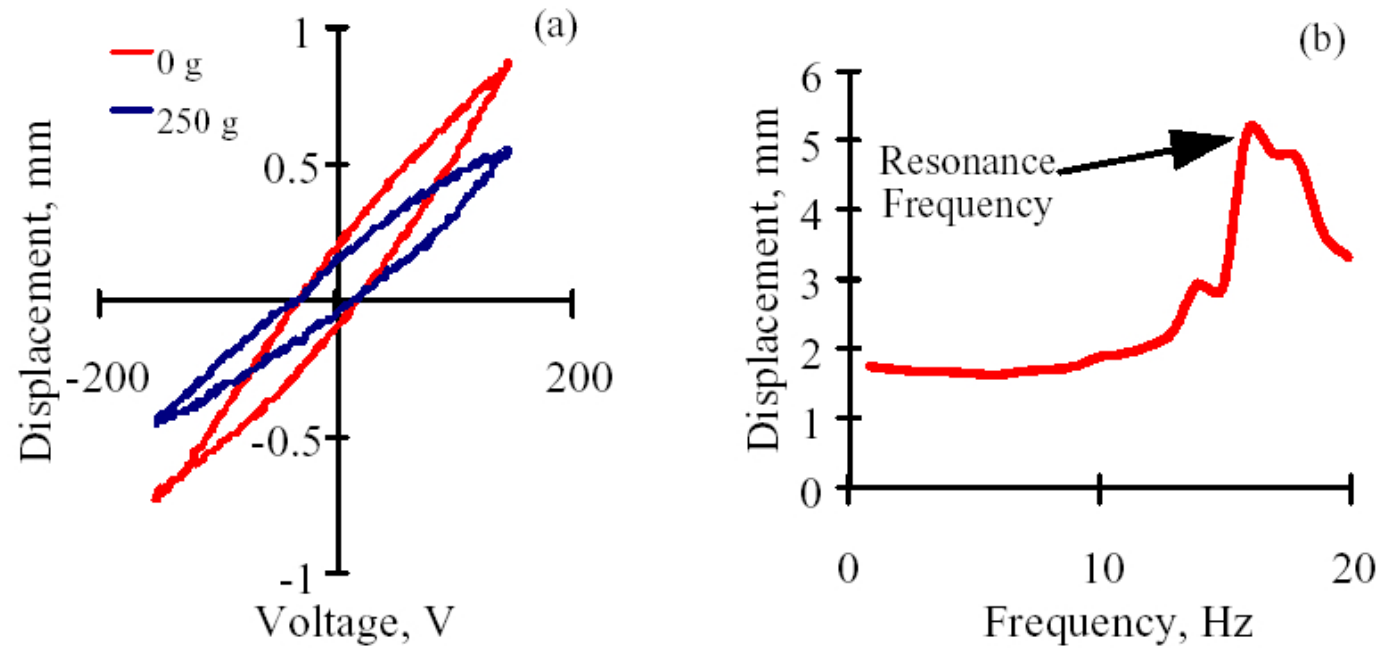

Figure 40 - The strain versus voltage curve and displacement performance for a THUNDER device (Simpson, 1998)

Tests have been conducted on a THUNDER device with $0.1-\mathrm{mm}$ thick stainless steel backing and a 0.2-mm thick PZT-5A ceramic layer. The actuator demonstrated a maximum of 1.7-mm total stroke with no load decreasing to approximately 1-mm total stroke with a $250-\mathrm{g}$ point load. The resonance frequency was found to vary significantly with clamping configuration and improved clamping methods are currently being developed application specific mounting configurations. (Simpson, 1998)

Currently most piezo-actuators do not possess the power or displacement for practical use in aircraft, but a new actuator called the Compact Hybrid Actuator does. In support of the Lockheed Martin MAS project a Penn State-Darpa team has developed a new piezoelectric actuator that uses a novel motion accumulation mechanism for folding and unfolding the wings of the MAS demonstrator.

(Figure

41 ,
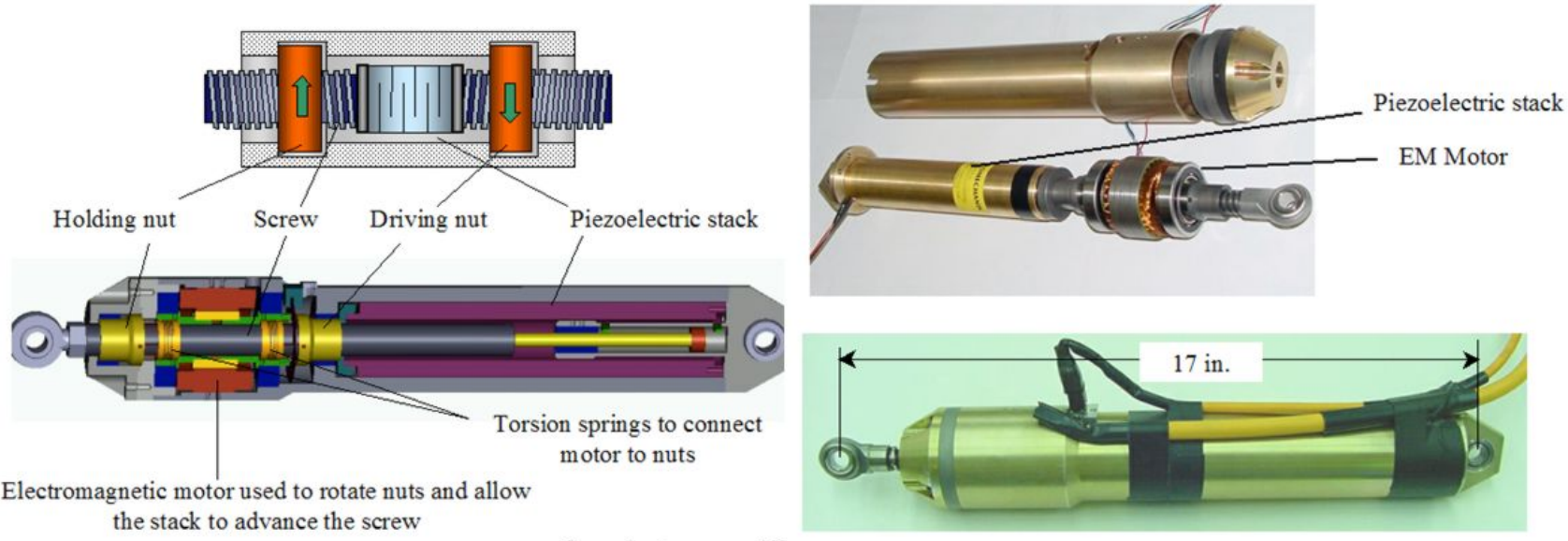
Figure $42, \&$ Figure 43) These actuators consist of a piezo-stack a lever arm and a reversible ratchet type drive, which is based on a 1967 patent by Breskend.

Main concept: Drive piezo material with high frequency electric field and accumulate small strokes to move a load

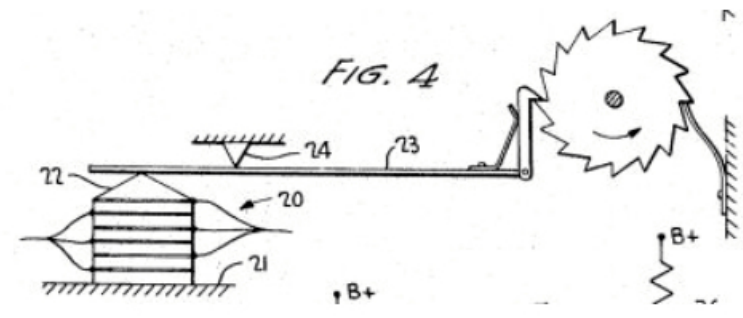

Breskend's Patent 1967

Figure 41 - Inspiration for the Penn State-Darpa Compact Hybrid Actuators Program (CHAP).(Koopman, 2004)

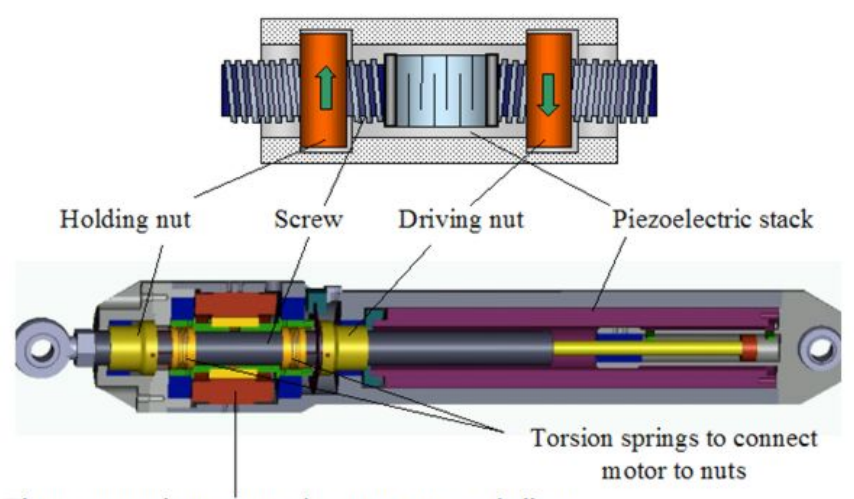

Electromagnetic motor used to rotate nuts and allow the stack to advance the screw

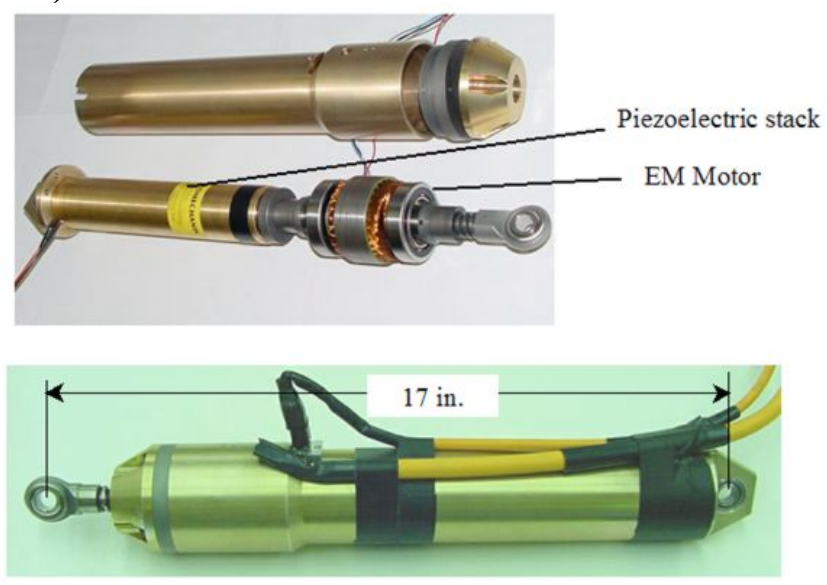

Figure 42 - Penn State CHAP Piezo-actuators (Koopman, 2004) 


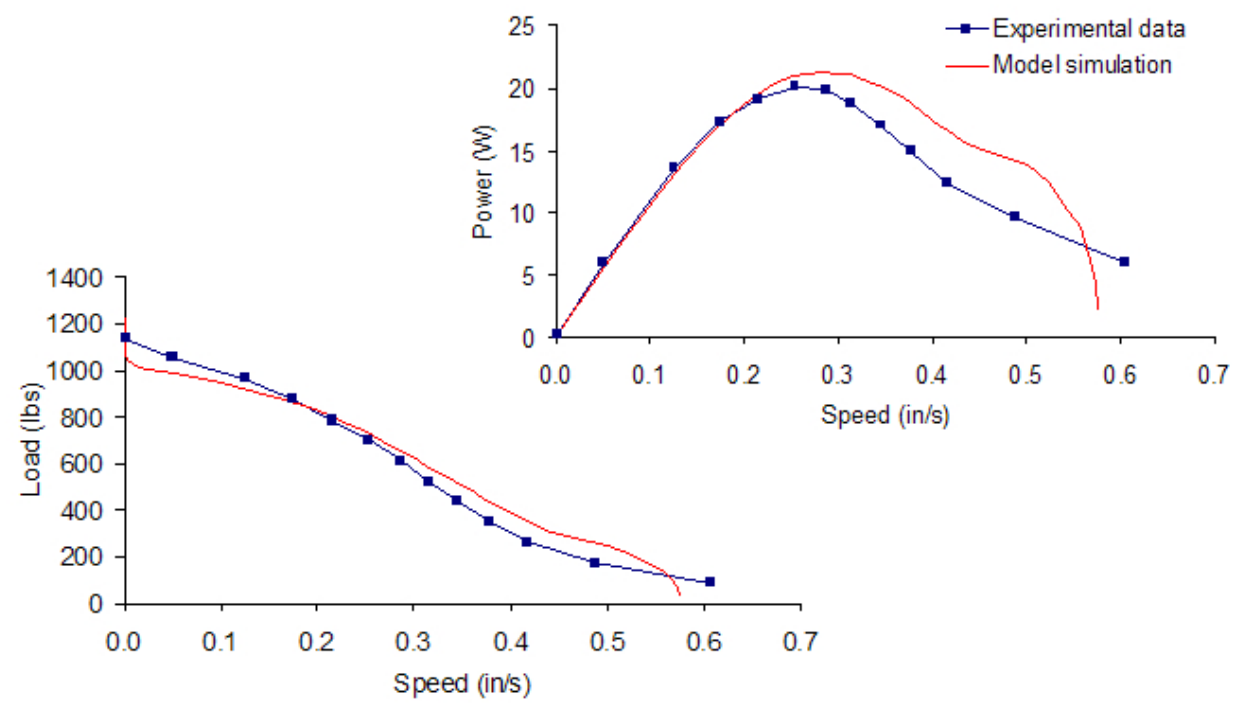

Figure 43 - Penn State CHAP Piezoactuator Load, Power velocity curves (Koopman, 2004)

The novel mechanism of the CHAP Piezo-actuator coupled with high frequency piezoelectric membranes gives the power and displacement needed for practical aircraft applications.

\section{Metallic shape memory alloys}

Shape Memory Alloys (SMA) are metallic alloys that are able to undergo reversible large deformations under loading/thermal cycles and are able to generate high values of thermal-mechanical driving forces. This very special behavior of SMA is due to their native capability to undergo reversible changes of the crystallographic structure, depending on the temperature and on the state of stress. These changes can be interpreted as reversible martensitic transformations between a crystallographic more-ordered parent phase, the austenite (A), to a crystallographic less-ordered product phase, the martensite (M). SMA can be produced as both wire and sheet. Passing a voltage through the SMA wire or sheet can generate the temperature change needed to produce a shape change. When the voltage is cut off the heat dissipates and the material returns to its original shape. SMA can be used alone as and actuator or incorporated into the matrix of a composite structure. Composites incorporating SMA are often referred to as smart composites. SMA use in smart composites is fairly new and is currently being studied by many research groups. (Sacco, 2004)

The Technical University Berlin has been conducting research into the use of embedded SMA wires in aircraft structures. They have constructed SMA controlled airfoil camber, winglets and motors. ( Figure 44), (Monner, 2002) 
Dr. Elio Sacco of the Department of Mechanics, Structures and Environment from the University of Cassino in Italy developing a micro mechanical model for the evaluation of overall constitutive behavior of a composite material obtained by embedding SMA wires into an elastic matrix. A simplified thermo-mechanical model for the SMA inclusion, able to reproduce the super-elastic as well as the shape memory effect, is proposed. It is based on two assumptions: the martensite volume fraction depends only on the wire temperature and the normal stress acting in the fiber direction; the inelastic strain due to the phase transformations occurs along the fiber direction. (Sacco, 2004)

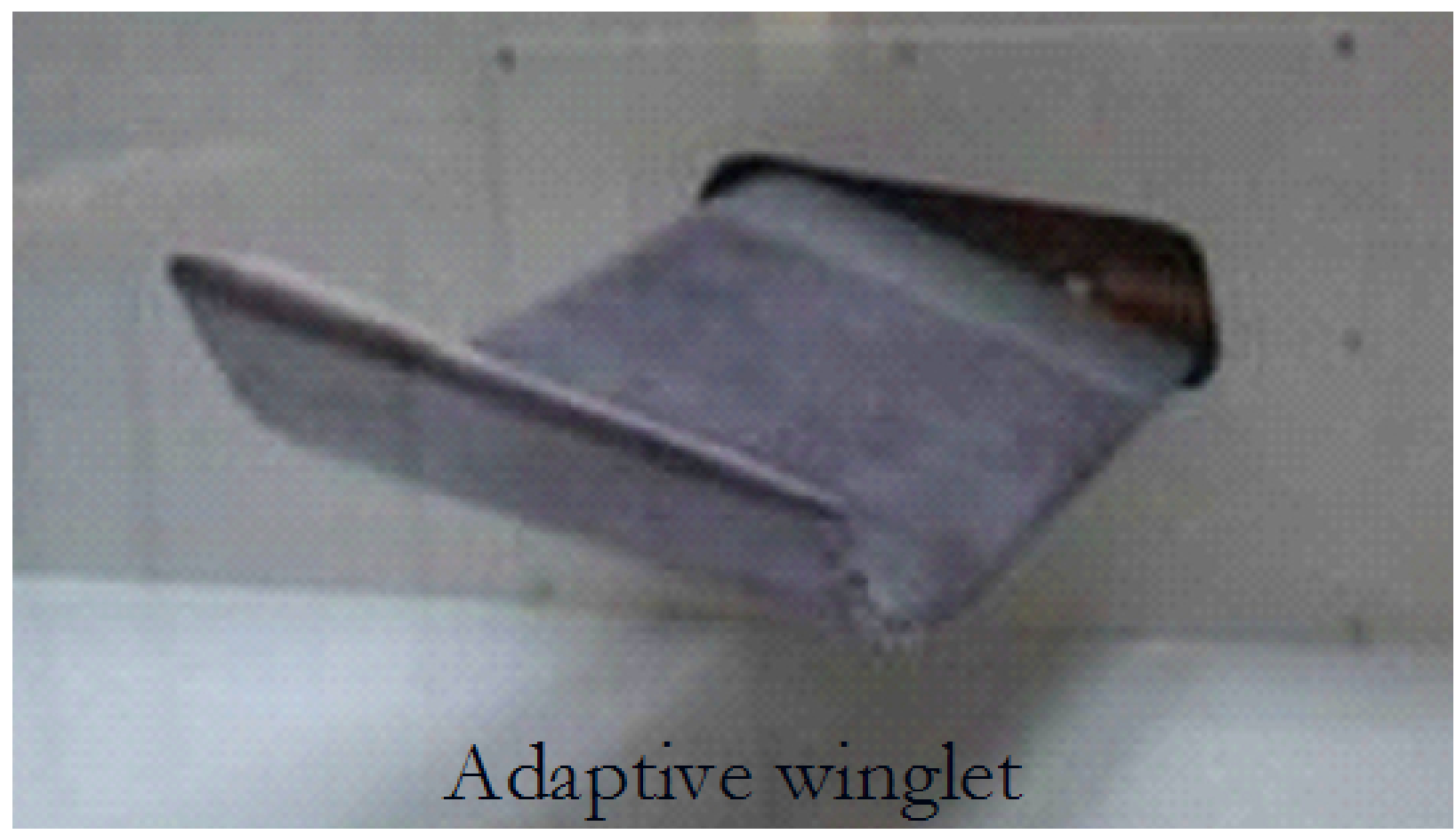

Figure 44- Smart composite wing model (Monner, 2002)

\section{Skins}

The skins on morphing control mechanisms are very important because aerodynamic efficiency requires smooth shape changes. The Wright brothers used French sateen fabric and NASA has used carbon reinforced plastic skins on the F-111 MAV and the F-18 AEW. The desired properties of the skin are dependant on weather the skin is an integral component of the aircraft structure as with the NASA aircraft or just a smooth fairing material.

There are a number of elastic skin materials available today to cover a morphing mechanism. Research at Virginia Polytechnic Institute has begun to characterize the mechanical properties of a variety of possible materials. The skin of a morphing structure should be elastic, flexible, have high strain recovery rates, resistant to weather conditions, resistant to abrasions and chemicals and have a hardness high enough to resist 
aerodynamic loads. The materials studied at Virginia Polytechnic were thermoplastic polyurethanes, copolyester elastomers, shape memory polymers and a variety of woven materials. (Kikuta, 2003)

The possible benefits of wing morphing are obvious for swept wing tailless aircraft. This technology offers us the opportunity to do away with the design compromises inherent for the control of these aircraft. Tailless aircraft will finally be able to approach the aerodynamic efficiencies envisioned by the pioneers in tailless aircraft. Enhanced control and maneuverability are also possible benefits of wing morphing.

Wing Morphing can immediately address the design compromise of using fixed washout or large winglets in swept wing tailless aircraft. Active morphing of the wing, variable washout, allows washout to be used only when maneuvering. This eliminates the corresponding drag for all non-maneuvering flight regimes. 


\section{Chapter 3 Research}

West Virginia University's Department of Mechanical and Aerospace Engineering initiated a project called "Control of a Swept Wing Tailless Aircraft through Wing Morphing" in 2003. This project is funded by NASA Dryden, West Virginia NASA Space Grant, Aurora Flight Sciences and the WVU Department of Mechanical and Aerospace Engineering. This project is focusing on improving the performance, handling characteristics, expanding the flight envelope and increasing the efficiency swept wing tailless aircraft using wing-morphing technology.

\section{Overview}

This research has focused on improving the handling characteristics, expanding the flight envelope and increasing the efficiency swept wing tailless aircraft using wing-morphing technology. This research has also allowed some insight into the flow physics which are generated around an adaptive washout morphing wing.

Phase 1 research allowed the experimental comparison of an existing state of the art tailless wing design with a design using wing morphing (adaptive washout). This phase also explored operational characteristics, handling characteristics, stall performance, and efficiency, of a swept wing tailless aircraft, utilizing wing morphing technology through the use of wind tunnel testing and UAV flight demonstrators.

Phase 2 of this research developed multiple CFD models that recreate the experimental aerodynamic data on the morphing design to better understand the flow physics. These CFD models have been used to explore the pressure and flow fields surrounding various morphed wing configurations.

\section{Objectives}

\section{Task 1 - Test gliders and RC flight demonstrator}

To design, Fabricate and test, gliders and an RC flight demonstrator to provide data for the design of conventional PUL-10 type wind tunnel models.

\section{Task 2 - Design and Fabricate Morphing Mechanism}

To design, fabricate and test a simple flight weight mechanism to morph (vary the washout) the outer $1 / 3^{\text {rd }}$ of a swept wing modeled on the PUL-10. This design is based on glider experiments and experiments using a $\mathrm{RC}$ wing morphing demonstrator experiments conducted in the $1^{\text {st }}$ quarter of this project

\section{Task 3 - Wind Tunnel Validation Model}

To utilize the 3D wind tunnel studies on a rectangular wing using the NACA 0015 airfoil conducted by Gerontakos and Lee in 2006 as a validation study for the wind tunnel aerodynamics and the force balance combination. 


\section{Task 4 - Comparison Wind Tunnel Testing}

To conduct experimental wind tunnel testing to compare the aerodynamics and control of the Horten PUL-10 (state of the art) with a design using the same planform and airfoil combination, but utilizing wing morphing (variable washout) on the outer $1 / 3^{\text {rd }}$ of the wing.

\section{Task-5 - CFD Model of adaptive washout}

To develop CFD models of the important control positions used by the morphing wing. The physical flow character in the tip region of the morphing wing is very complex and is responsible for the unique behavior, which lead to a minimum drag corresponding with a maximum lift. While the reasons for this are understood for the conventional elevon equipped wing through theory, as due to the rotation of lift vector due to the wing twist, up-wash and cambered airfoil created by a control deflection, this phenomenon is not understood for the morphing wing. CFD gives us a more complete view of the flow physics.

\section{Task-6 - Flight Testing of adaptive washout flight demonstrator}

To design and construct a flight weight wing morphing mechanism and collect actual free flight aerodynamic data and analysis of an actual flight weight adaptive washout morphing mechanism are very important. An Eagle tree flight data recorder will be used $\mathrm{f}$ in order to document actual climb rate, roll rate, glide angle $\left(\mathrm{C}_{\mathrm{D}}\right)$, maximum velocity, and stall speed and other relevant performance.

\section{Task-7 Compilation of Data}

Data from CFD, wind tunnel testing and actual flight data will be combined in order to ascertain the performance of adaptive washout morphing mechanism and in order to more fully understand the flow phenomena encountered with a morphing swept wing tailless aircraft.

\section{Parallel Work}

There are a number of studies pertaining to morphing aircraft structures as of 2007, but the studies most closely related to this project are as follows: NASA Langley - Morphing Project with biologically inspired geometries.(Cancelled), NASA's Dryden Flight Research Center F-18A Aero-elastic Wing Demonstrator, Lockheed Martin - MAV and Polecat UAV programs, University of Florida - Morphing urban tactical UAVs, WVU Masters Thesis - Ashwin Vishwanathan - Potential Flow modeling, WVU Masters Thesis -Chris Griffin - Mechanized skin study. 


\section{Geometry / Aerodynamics}

The WVU project begans with the assumption that the Horten-Panek PUL 10 is a state-of the art Horten type swept wing tailless aircraft. Designed by Siegfried Panek and Dr. Riemar Horten, the PUL-10 is a very efficient well handling two place tailless general aviation aircraft. This design was used as a basis for the aircraft aerodynamics in this project. The basic planform, controls and washout were a starting point and then adapted based on scale considerations, remote control aircraft conventions and fabrication-based constraints. Through the process of developing a wing morphing mechanism for the control of a swept-wing tailless aircraft comparisons in performance will be made back to PUL-10 type wind-tunnel model.

The airfoil combination of the PUL-10 has been modified from the original to a modified Horten II airfoil called the MH-78 at the root and the NACA 0010 at the tip. The MH-78, designed by Martin Hepperle, and has better performance then the original Horten airfoil at the low Reynolds numbers associated with the wind tunnel models and the flight demonstrators. (Hepperle, 2004) This airfoil has been designed for light, man-carrying gliders, with moderate wing sweep. Maximum lift capabilities and good handling qualities were the most important design requirements. The tip airfoil was changed from the Horten Symmetric 10\%, to the NACA 0010. The shape and performance of these airfoils are almost identical. The NACA 0010 was chosen because it is widely used and there is a wealth of literature written about its performance. The well-understood behavior of this airfoil may be beneficial in the advanced stages of this research.

The PUL-10 was designed with a sweep of 35 degrees and washout combination to develop a bell shaped (lift distribution proportional to $\left(\sin ^{3} x\right)$ ) lift distribution to counteract adverse yaw during maneuvering. The non-morphing comparison model's washout was simplified to allow available construction techniques to be used in the construction of the models. Four degrees of dihedral was built into the models for stability. (Figure 45) 


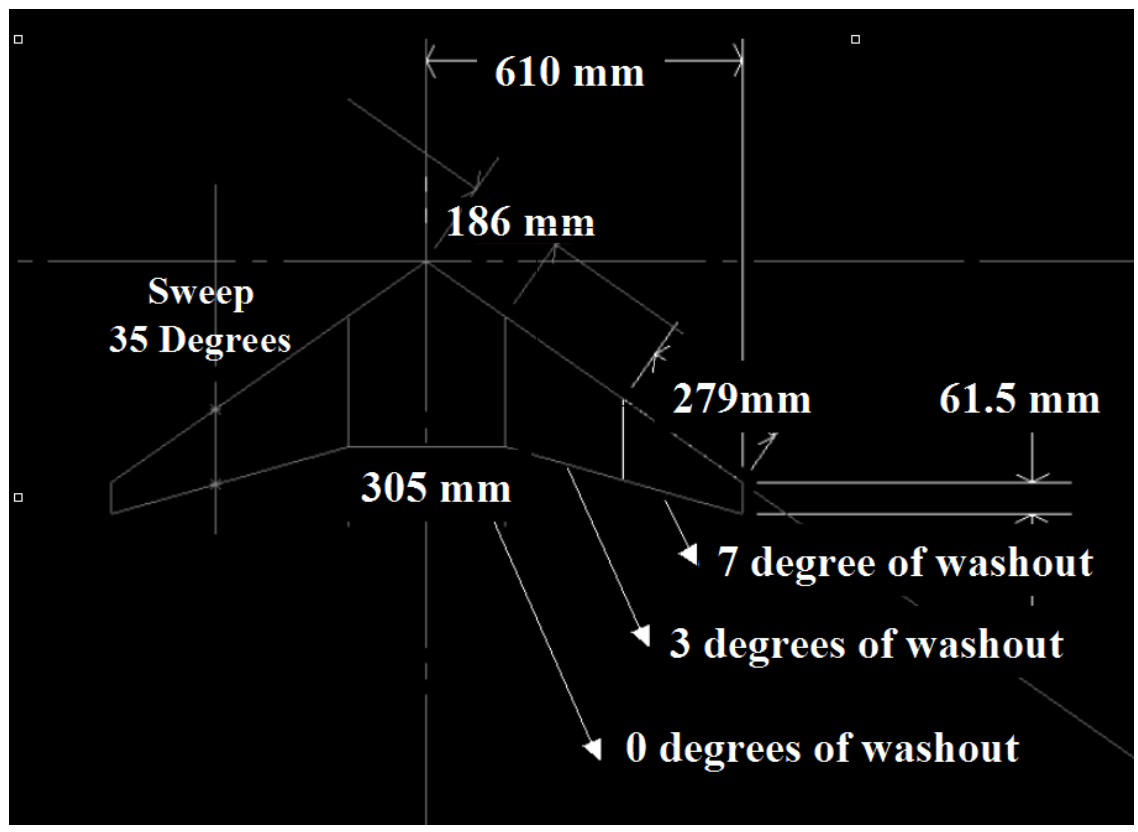

Figure 45 - Final basic aerodynamic Non-morphing configuration with dimensions in inches and sectional washout angles.

Glider and RC model testing with the aerodynamic configuration shown in Figure 45 were used to collect performance data to compare to the morphing models.

\section{Morphing mechanism development}

Study of early tailless designs and approximation techniques led to the conclusion that varying the twist in the outboard sections of a wing can develop the desired control forces needed for maneuvering flight. The Wright brothers demonstrated this with their wing warping controls; this also has been proven by mathematical models used by the Horten's and Karl Nickel that modeled control force changes as changes in twist. Most of the twist and control surfaces in Horten aircraft are located in the outer $1 / 3$ of the wing, so it was decided to in the current work allow the entire outer $1 / 3$ of the wing to change twist in the initial morphing models. This area will be optimized after initial test results are studied.

There are two requirements of a morphing wing structure, which are opposed to each other. The first is that the structure needs to be flexible enough to allow significant shape change with minimal actuation forces. The second requirement is that the wing structure needs to be rigid enough to maintain a predictable aerodynamic shape in the presence of aerodynamic loads.

A common problem with aircraft wing structures is flutter, which is an oscillation caused by aerodynamic forces. This is a much more serious problem with aircraft that have very light-weight structures or high aspect ratio wings. The Horten brothers documented flutter problems on the Ho-IV. The Ho-IV has an aspect ratio of 32.4 and a span of 24.4 
meters. The wing structure is mostly of wood with a limited number of magnesium components. They identified two speed ranges, which they just had to hold-on and fly through where flutter was severe. (Bowers, 1998) Even the F-18 has flutter modes during maneuvering. (Brenner, 1997) These two examples are for aircraft structures that were more or less designed to be rigid. The design of a morphing structure which must be rigid at all times yet is free to assume a new shape with a reasonably sized actuation force is a major problem. There are a number of new actuator technologies, which offer some help in this matter, Piezo-electric membranes or Shape Memory Alloy actuators. When these actuator technologies are embedded in a composite structure they may solve the structural problems of morphing aircraft, but both technologies are very immature, lack the range of motion needed for the power available, and are currently cost prohibitive.

The WVU first generation morphing design depended on a composite skin and a carbon actuator rod to provide the resistance to aerodynamic forces. During the morphing process the composite skin was forced to assume a new wing twist. Morphing was initiated using a carbon actuator rod and a geared high torque servo. Rigidity was built into the leading edge of the wing skin with unidirectional carbon tape. This rigid zone acted as an axis of rotation during wing morphing. (Figure $46 \&$ Figure 47)

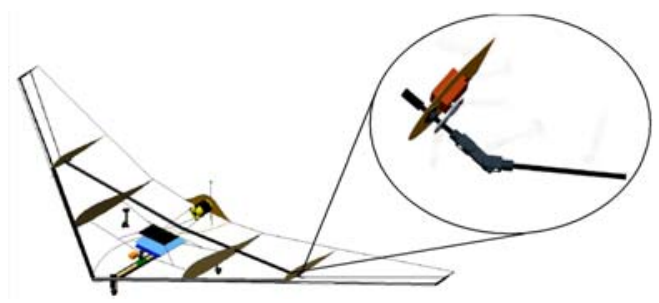

Figure 46- First generation actuator and torque rod geometry for morphing control.

The skin and trailing edge were left free to assume a new twist. The composite skin of graphite and fiberglass had been designed through experimentation to assume close the desired shape during morphing by positioning the ply drop offs and unidirectional carbon where rigidity was needed.

The first model was a $1220 \mathrm{~mm}$ semi-span model. The skins where constructed of $3 \mathrm{k}$ woven graphite cloth, $2.4 \mathrm{oz}$ woven glass cloth and $3 \mathrm{k} 5.7 \mathrm{oz}$ carbon unidirectional tape. Various skins were laid-up, constructed and then placed in a wind tunnel to determine the minimum structure needed to resist aerodynamic forces at the expected maximum flight speed of $30 \mathrm{~m} / \mathrm{s}$. The morphing mechanism used $15.8 \mathrm{~mm} \mathrm{O.D.} \mathrm{wrapped} \mathrm{carbon} \mathrm{tubing}$ as guide rods and $6.3 \mathrm{~mm}$ O.D. pultruded carbon rods as torque rods. The torque rod required a $16 \mathrm{~kg}-\mathrm{cm}$ high torque servo with a 2:1 gear ratio for actuation. This design was then scaled down to a $610 \mathrm{~mm}$ semi-span wing for wind tunnel testing. 


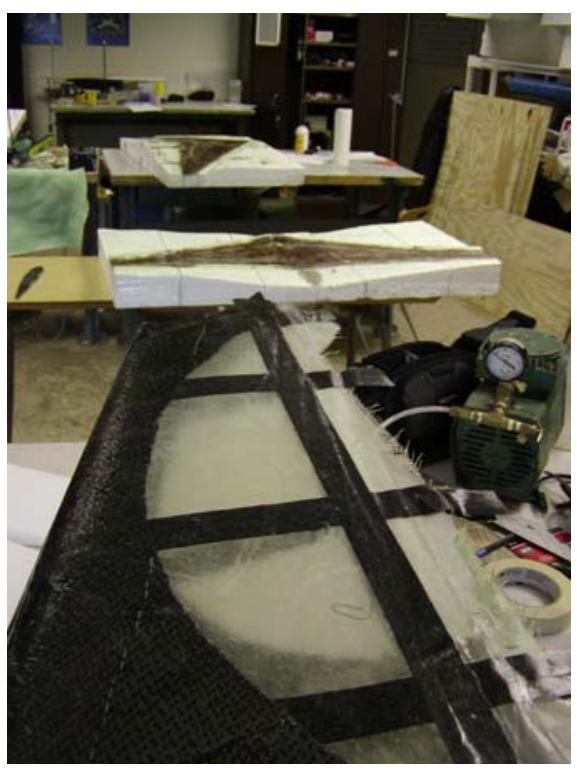

Figure 47 - First generation composite skin made of woven glass and carbon and unidirectional carbon.

The first generation adaptive wash out morphing mechanism showed a number of areas for improvement. The following are problems with the first generation design: 1. Even when the trailing edge skins remained unbonded there were unwanted shape changes (buckling) on either the top or bottom of the wing due to compression and twist during washout changes, 2. Repairs or modifications to the morphing mechanism were impossible once the assembly was bonded together and 3. Actuation forces were greater than a standard high torques servo could provide.

The above problems made research modifications difficult and created the inability to maintain the desired airfoil shapes. Multiple test wing skins and a preliminary wind tunnel model were constructed. Experience from these structures led to a new design, which solved the above problems. Figure 47 shows the first generation design composite morphing wind tunnel model in blue with obvious buckling and unwanted shape changes.

Tests on the first generation models indicated that there needed to be an elastic skin, which could deal with significant shape and area changes and that there needed to be 


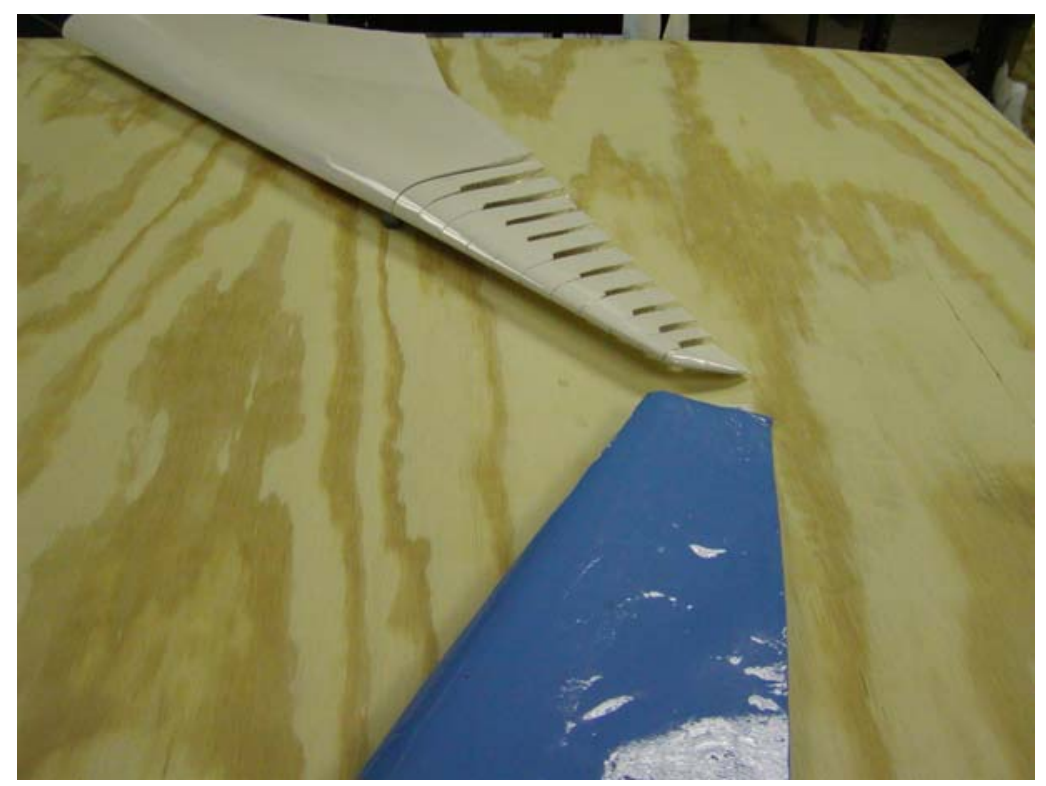

Figure 48 - WVU 1st Generation $610 \mathrm{~mm}$ span wind tunnel model in blue, showing obvious skin buckling.

airfoil shaped ribs or wing sections beneath the skin to maintain the desired airfoil shapes. The new design utilizes a rigid carbon tube and torque rod in the leading edge. The torque rod has a 90-degree bend near the wing tip with a $50 \mathrm{~mm}$ section extending toward the trailing edge. This $50 \mathrm{~mm}$ section is permanently attached to the tip rib wing section. There are 10 free-floating wing sections from the tip rib wing section toward the wing root. These free-floating wing sections are connected at the trailing edge by a spring loaded $2 \mathrm{~mm}$ Teflon coated cable. When the tip rib wing section is rotated the retaining cable pulls each wing section in rotation around the $3 \mathrm{~mm}$ torque rod in the leading edge. A $0.25 \mathrm{~mm}$ latex skin then covers this entire assembly. Figure 48, Figure 49, Figure 50 \& Figure 51 show an uncovered partially disassembled $2^{\text {nd }}$ generation morphing mechanism. 


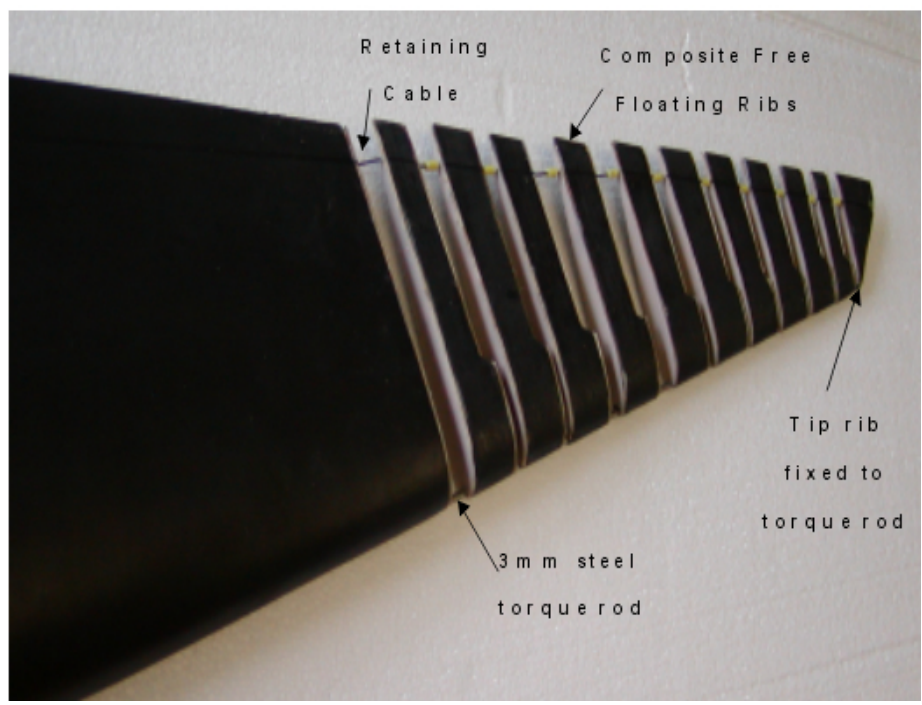

Figure 49 - Partially assembled second generation morphing wing showing the ribs, retaining cable and torque rod.

Each free-floating airfoil section has $6 \mathrm{~mm}$ of material relieved from the aft two thirds of one side. This allows the latex skin to form a smooth curve fit when the torque rod and the last wing section are rotated (washout varied). Simply varying the spring tension on the retaining cable adjusts the wing rigidity (flutter resistance). The cable tensioning mechanism can be seen in Figure 50.

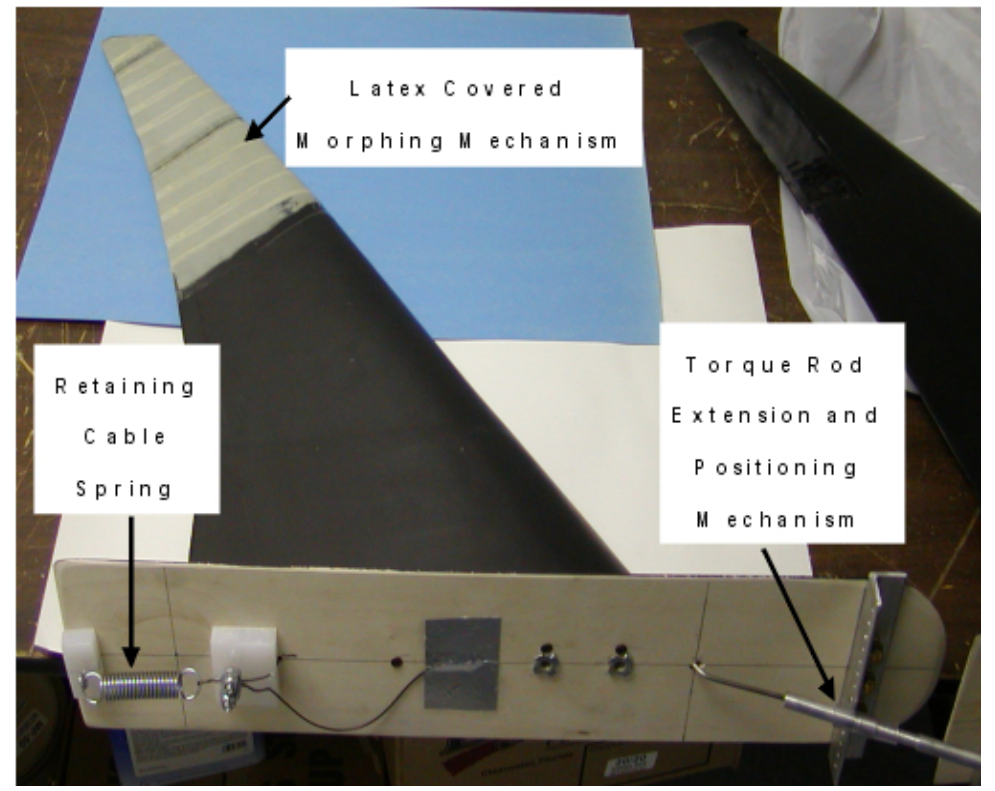

Figure 50- Morphing wind tunnel model showing the latex skin, the tensioning mechanism and the positioning mechanism. 


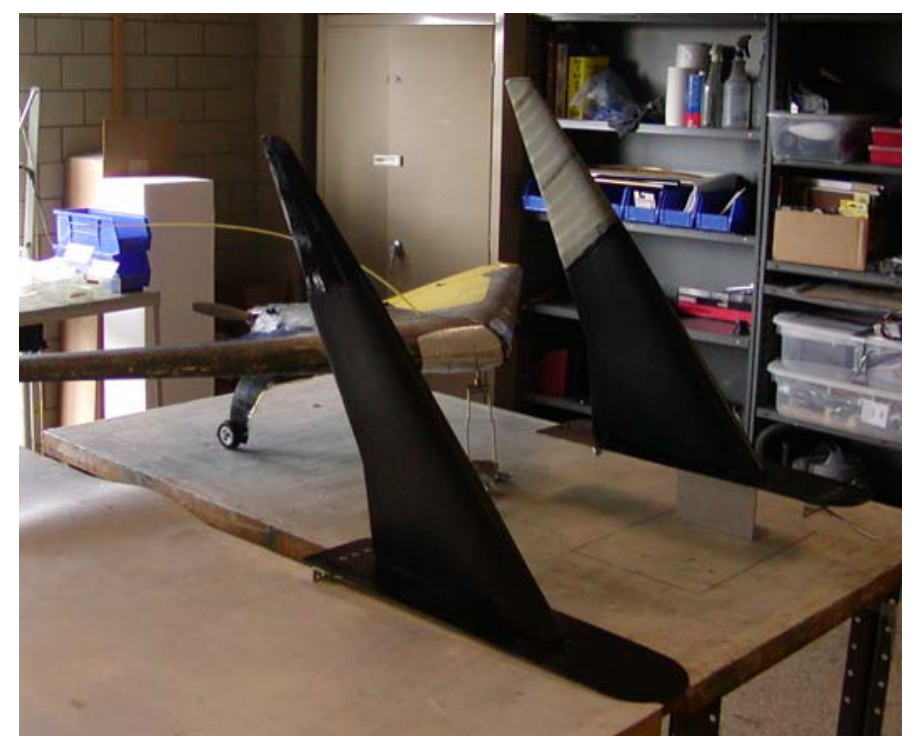

Figure 51-Completed morphing and non-morphing wind tunnel models.

When fully assembled the morphing wing's outer $33 \%$ is able to change twist either manually, as with the wind tunnel models, or by using a high torque servo. Actuation torque on the torque rod varies between $0.1 \mathrm{~N}-\mathrm{m}$ and $0.35 \mathrm{~N}-\mathrm{m}$ depending on angle of twist desired in the wing. Possible tip rotation angles around the leading vary between 15 degree and +10 degrees. Figure 53 shows the wing tip in position 3 that corresponds to a tip rotation of +7 degrees. Figure 54 shows the corresponding position 3 used for testing on the elevon comparison model actual morphing tip deflections and elevon deflections are plotted in Figure 55. Because the leading edge of the morphing wing remains fixed the deflection of the trailing edge indicates the amount of twist in the wing section. The trailing edge deflections for the 5 test control positions can be seen in Figure 55.

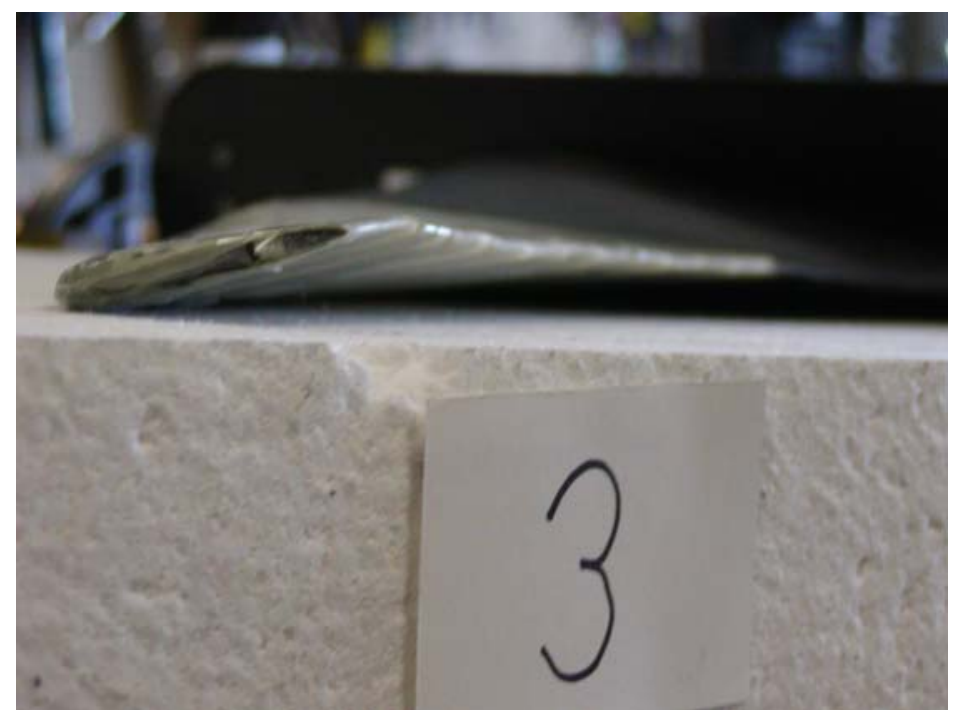

Figure 52-Control position 3 in the morphing wing which corresponds to a +7 degree twist of the tip. 


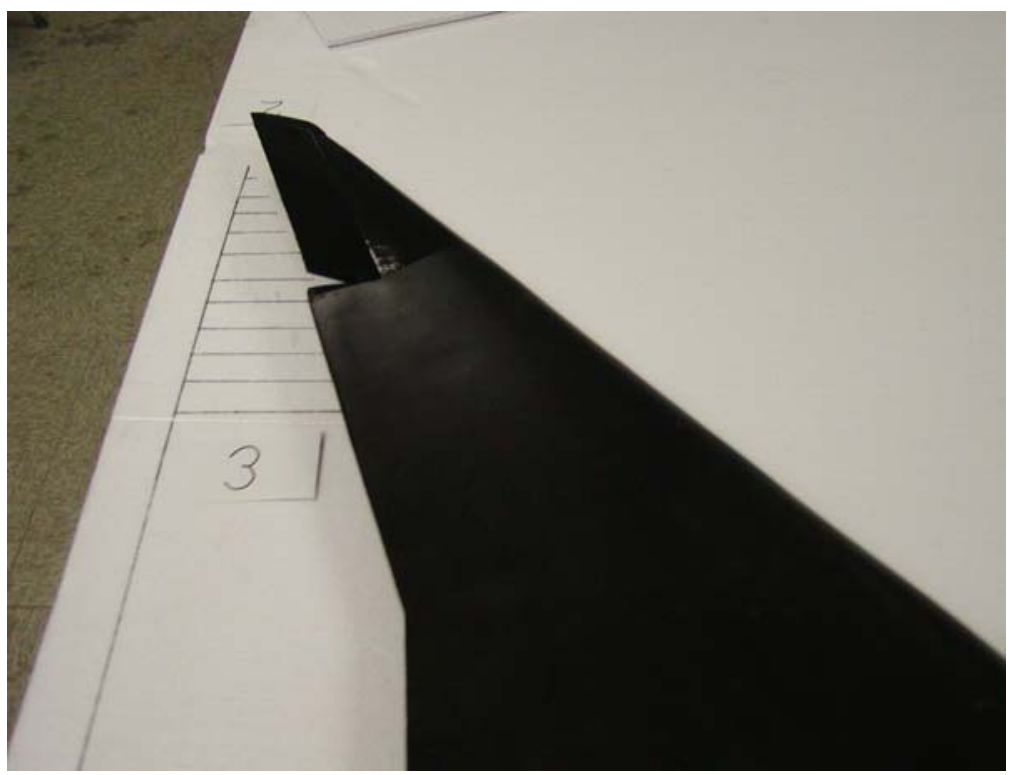

Figure 53- Control position 3 as seen on the elevon equipped comparison wind tunnel model.

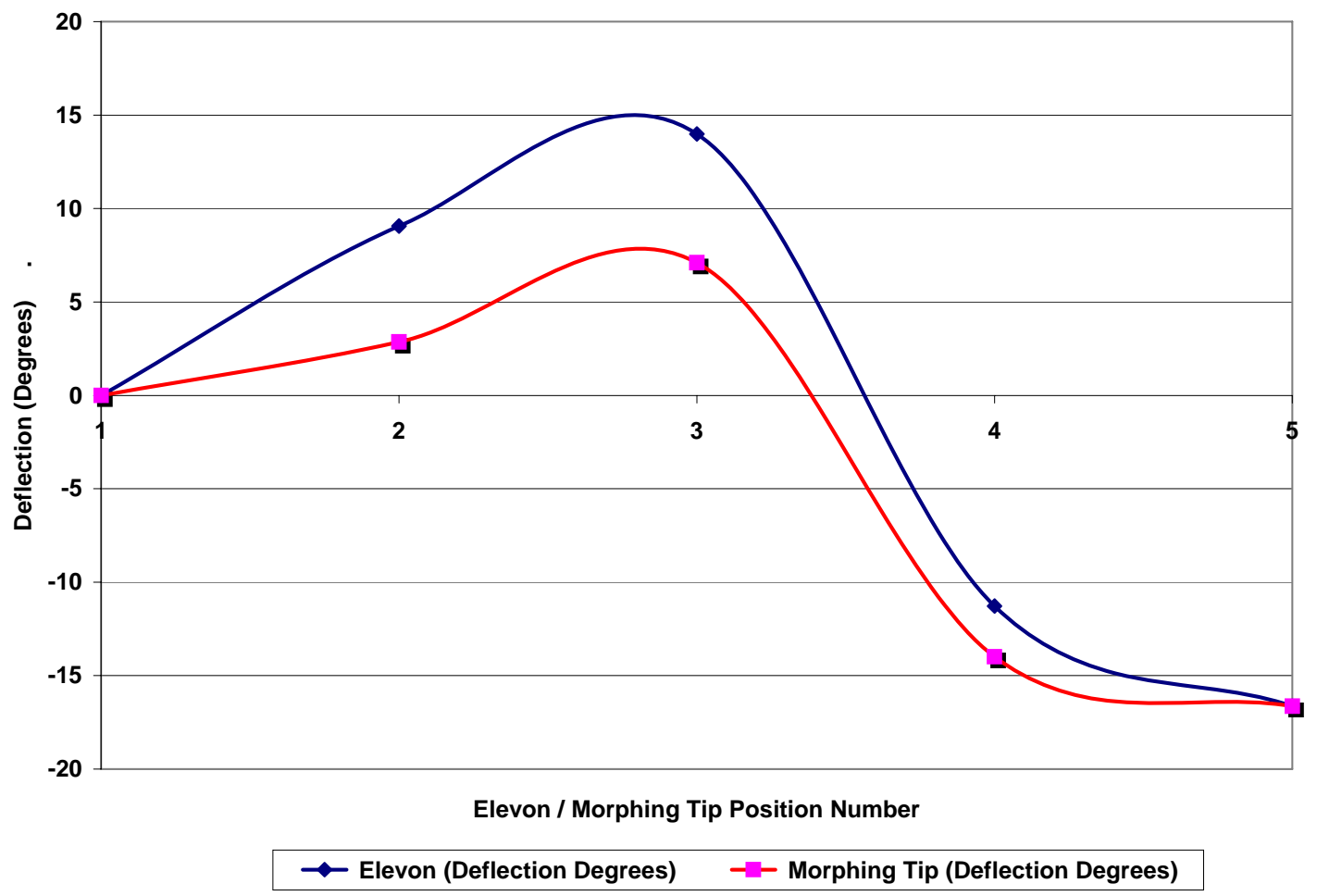

Figure 54-Comparison of initial model elevon deflection angle and initial Morphing wing model tip rotation angle for the 5 test control positions. 


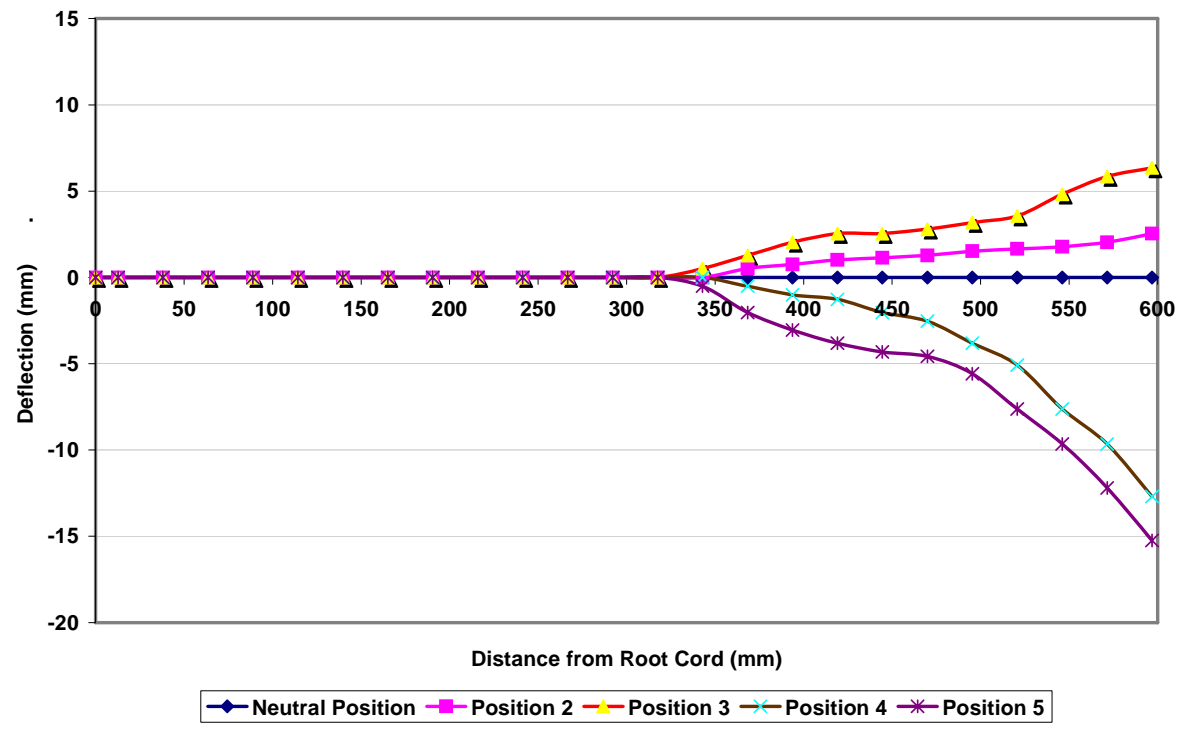

Figure 55-Morphing wing trailing edge deflections for the 5 test control positions.

Both the elevon equipped comparison model and the morphing wing wind tunnel models were then tested in the Vigyan low speed wind tunnel located next to NASA Langley Research Center in Hampton, Virginia. Aerodynamic and control forces were measured at 5 different control positions for each wind tunnel model at a realistic UAV demonstrator velocity of $18.6 \mathrm{~m} / \mathrm{s}$. Figure 56 shows the morphing wind tunnel model installed in the wind tunnel in it's neutral control position. Figure 57 and Figure 58 show the measured control force and moment coefficients for both models at each of the 5 test control positions at 0 degree AOA.

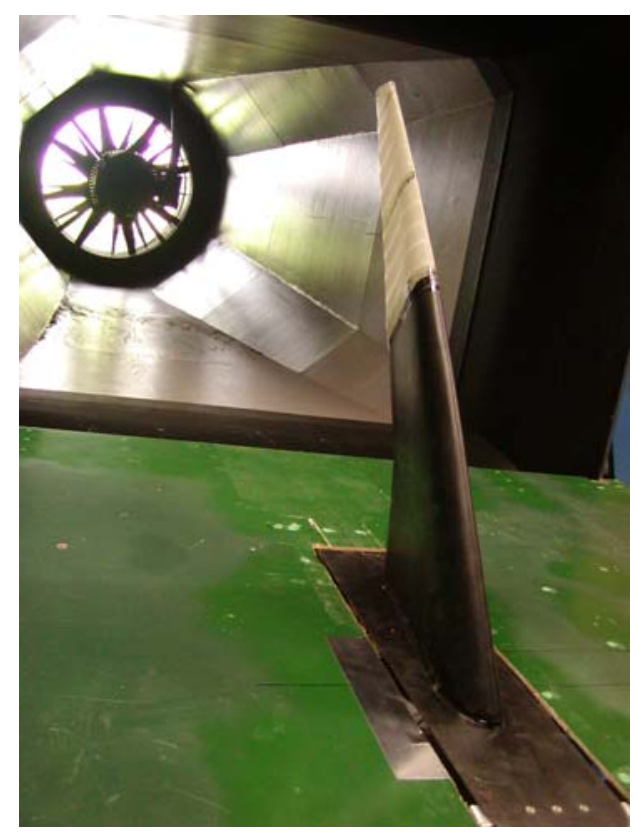

Figure 56- Morphing wind tunnel model installed in the Vigyan wind tunnel in Hampton Virginia. 
The morphing wing was tested at wind tunnel velocities from $12 \mathrm{~m} / \mathrm{s}$ to $45 \mathrm{~m} / \mathrm{s}$. Oscillations in the lift direction or flutter became apparent after a wind tunnel velocity of approximately $20 \mathrm{~m} / \mathrm{s}$. Actual tip deflection in these oscillations increased to about + or $10 \mathrm{~mm}$. Using a digital oscilloscope each force and moment channel was observed. The flutter was most apparent in the roll moment channel of the force balance with an average frequency of $9.33 \mathrm{~Hz}$.

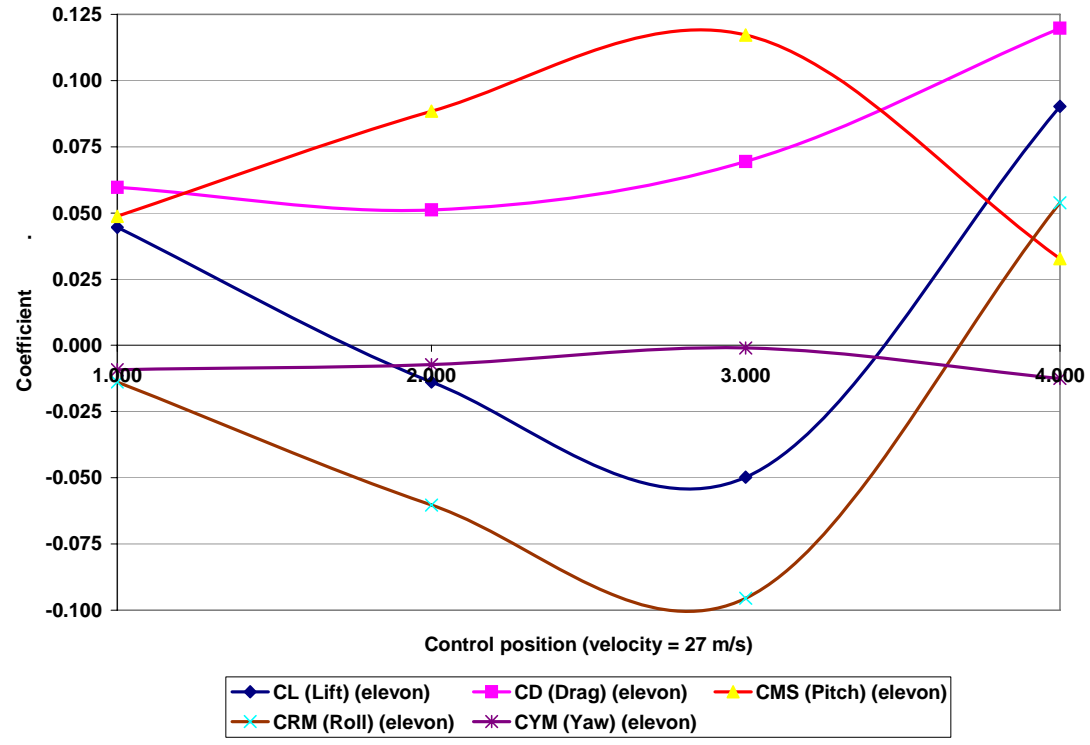

Figure 57- Early Elevon equipped wind tunnel model control forces and moments at 5 test control positions.

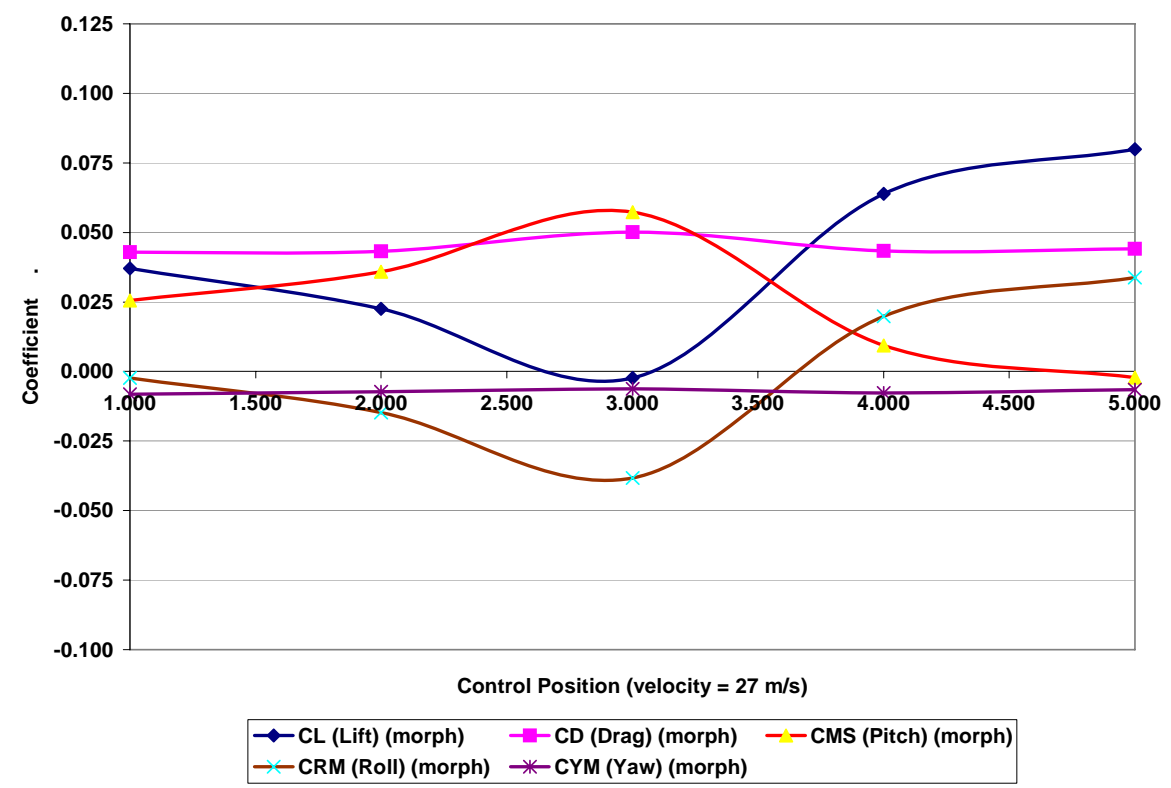

Figure 58-Early Morphing wind tunnel model control forces and moments at 5 test control positions. 
Both wind tunnel models were also tested at wind tunnel velocities between $18 \mathrm{~m} / \mathrm{s}$ and $27 \mathrm{~m} / \mathrm{s}$ in a cruise (neutral) control positions configuration. Even though the tests were conducted with the wing at a zero angle of attack the corresponding lift to drag ratios for the morphing wing were almost $15 \%$ better then the elevon-equipped wing with its associated built in washout.

\section{Morphing Mechanism Improvements}

Initial wind tunnel testing indicated that a number of improvements needed to made to the morphing wing mechanism. In an attempt to reduce flutter and improve the operation of the adaptive washout morphing mechanism each of the free floating wing section had a $20 \%$ increase in the bearing area between them and the other wing sections. The bearing surfaces where covered with a 10 thousands Teflon ${ }^{\mathrm{TM}}$ film. After nine wind tunnel testing sessions the latex skin was beginning to tear at all of the trailing edges of the free floating wing sections. (Figure 59 \& Figure 60) To remedy this, the sharp trailing edges were rounded and reshaped. To improve both durability and ability to resist ballooning at low pressures found on the upper wing surface the 10 thousands of an inch Latex skin was replaced by a 14 thousands of an inch Neoprene skin. (Figure 61) The Neoprene had the desired effect but introduced a new fabrication problem. Effective adhesives did not have the same elasticity as the neoprene and cracks developed in any region where adhesive had been applied. An adhesive which remained pliable yet has the strength needed to hold a wing skin seam together. A variety of epoxies, neoprene glues, cyanoacrylates, rubber cements and silicon adhesives were tested on $4 \mathrm{~cm} \times 8 \mathrm{~cm}$ neoprene test coupons. An improvised peel test was conducted to measure the bond strength and the test coupons were stretched by $20 \%$ in length 10 times and then observed for cracking. The solution turned out to be a combination of rubber cement bonding a $1 \mathrm{~cm}$ over-lap with the edge then sealed with silicon neoprene seam sealer.

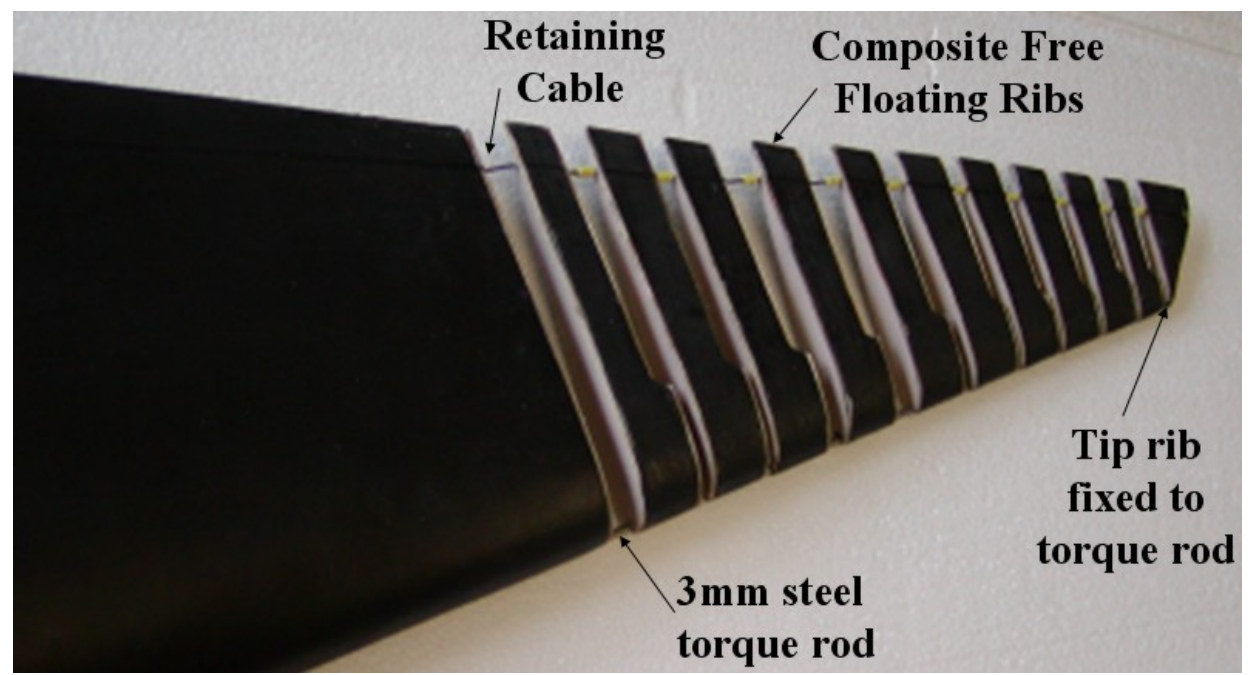

Figure 59 - Basic modified adaptive wash-out mechanism structure. 


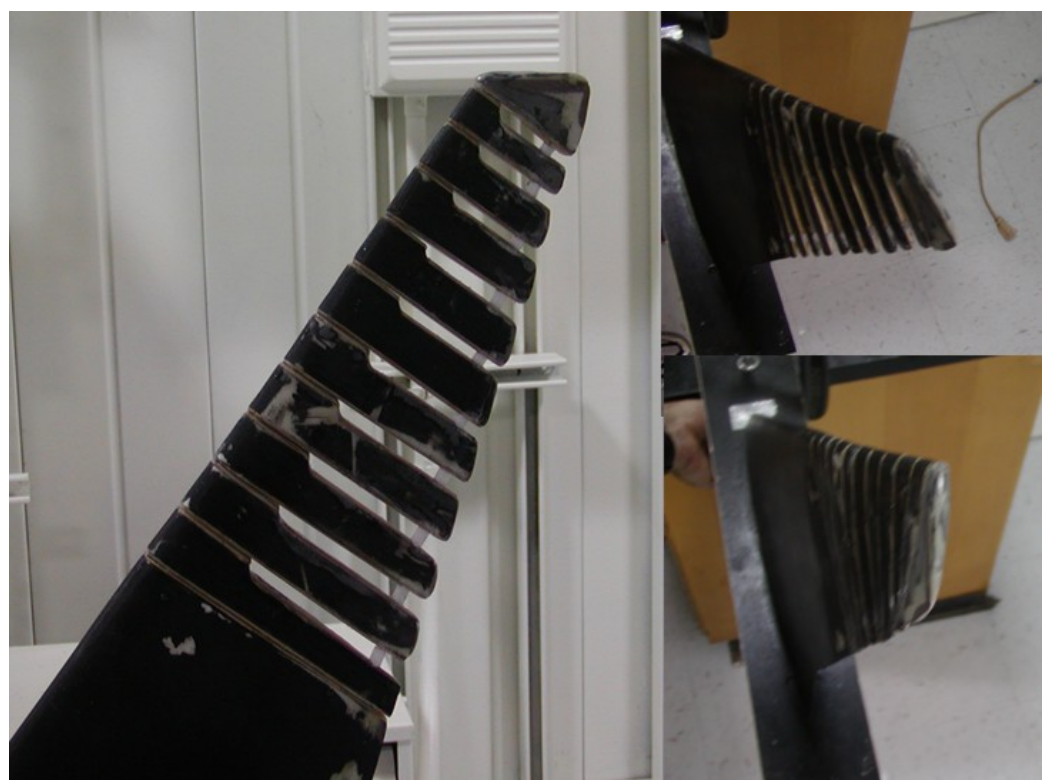

Figure 60- Spring 2006 modifications to the adaptive washout mechanism which include rounding of trailing edges, new Nylon restraining monofilament and Teflon spacers between trailing edges of each free floating wing section.

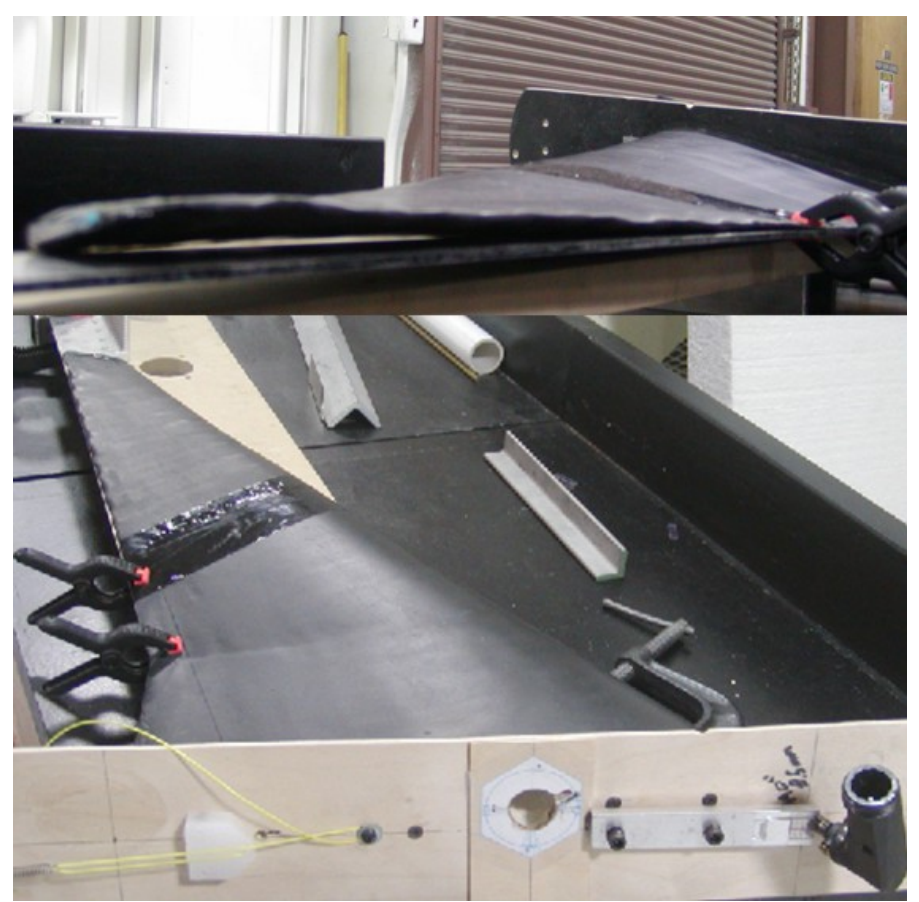

Figure 61- Skinned morphing wing, Yellow 0.050 inch monofilament, 0.014 inch Neoprene skin and control position adjustment mechanism can be seen.

Improving the actual shape of the morphing portion of the wing during control changes was a goal when it was decided to replace the 0.050 inch Teflon ${ }^{\mathrm{TM}}$ coated steel restraining cable in the wing trailing edge with a 0.050 inch Nylon monofilament. The Nylon monofilament has better elastic and friction properties then the steel cable. This replacement smoothed out the curvature of the trailing edge during morphing and was responsible for a $12 \%$ drop in needed actuation torque. The improved adaptive washout 
wind tunnel models can be seen in Figure 59, Figure 60 \& Figure 61 . The morphing portion of the wing assumed slightly different shapes with the 5 control positions than previously. The new trailing edge shapes can be seen in Figure 62 at control position 1 (neutral tip), control position 2 (-3.5 degree tip deflection), control position 3 ( -7 degree tip deflection), control position 4 ( +3.5 degree tip deflection), control position 5 ( +7 degree control position.

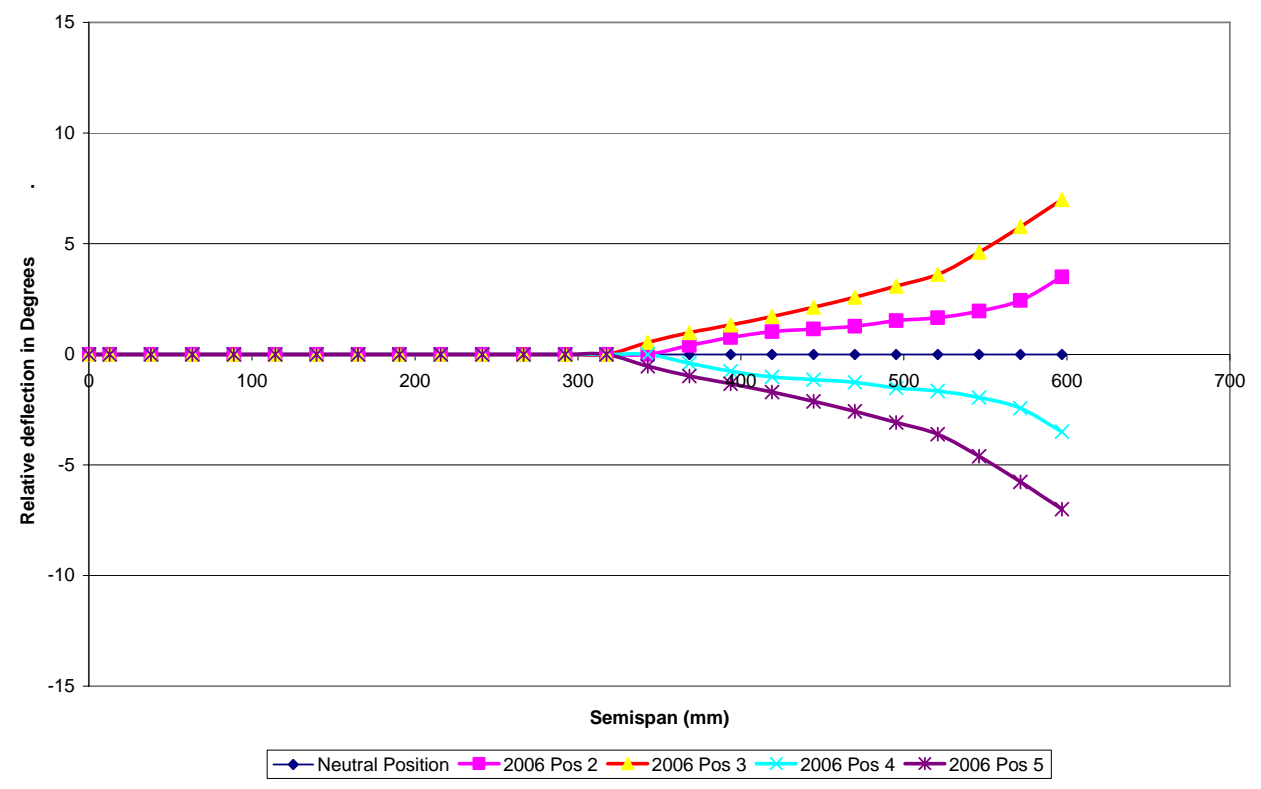

Figure 62- Trailing edge curvature with in Nylon monofilament. Note Leading edge remains fixed.

The elevon equipped PUL-10 type wing remained unchanged from 2005 except for new control settings. The five control positions on both models were adjusted so that either the elevon or the tip of the morphing wing were at $0,-3.5,-7,3.5$ and 7 degree AOAs from their neutral position. (Figure 63) Elevon AOA was measured from the hinge line aft and on the morphing model tip deflection was measured from the leading edge aft.

Table 2 - Elevon deflection for Horten type wing

\begin{tabular}{|c|c|c|}
\hline $\begin{array}{l}\text { Control } \\
\text { Position }\end{array}$ & $\begin{array}{c}\text { Elevon } \\
\text { Deflection } \\
\text { Degrees }\end{array}$ & $\begin{array}{c}\text { Morphing } \\
\text { Wing Tip } \\
\text { deflection } \\
\text { degrees } \\
\end{array}$ \\
\hline 1 & 0 & 0 \\
\hline 2 & 3.5 & -3.5 \\
\hline 3 & 7 & -7 \\
\hline 4 & -3.5 & 3.5 \\
\hline 5 & -7 & 7 \\
\hline
\end{tabular}



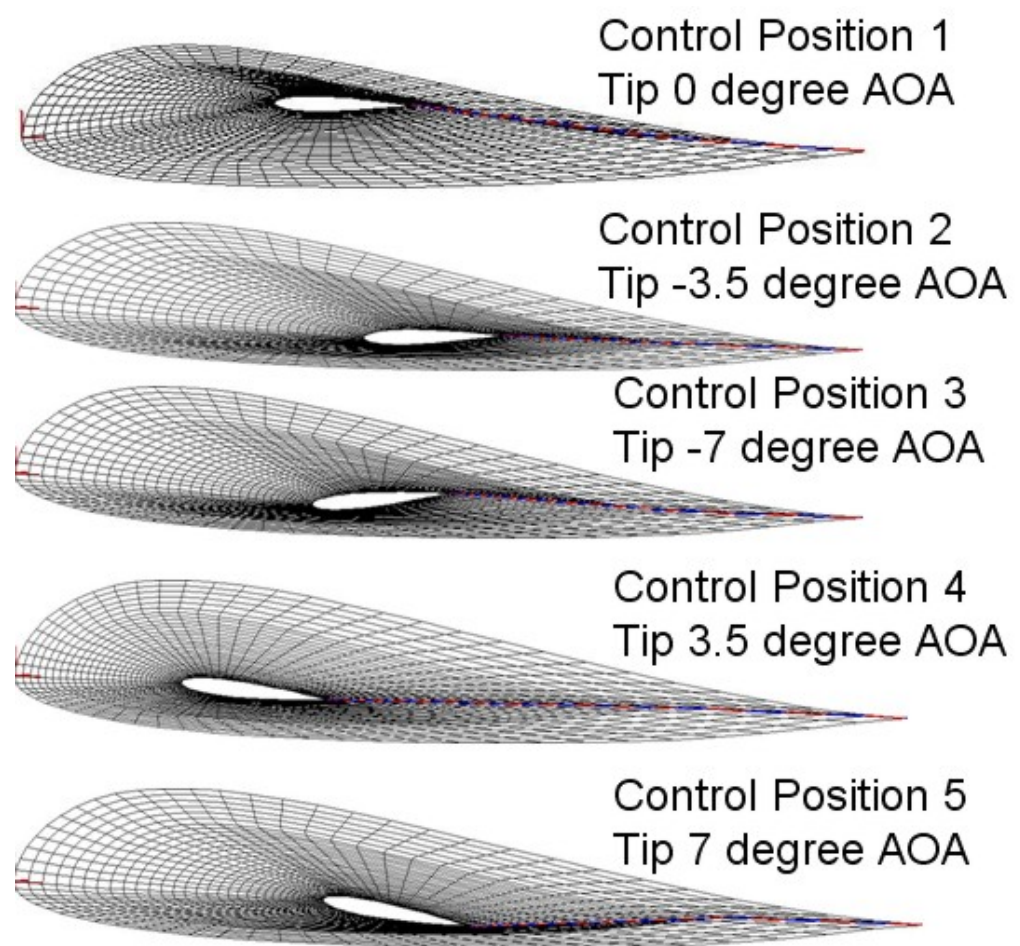

\section{Morphing Wing Control Positions}

Figure 63 - Schematic of side view of morphing wing at control positions 1-5.

Angle of attack for both of the wind tunnel models was changed by rotating the models around the force balance and reattaching the models at the desired angle of attack using pre-machined screw holes. (Figure 64)

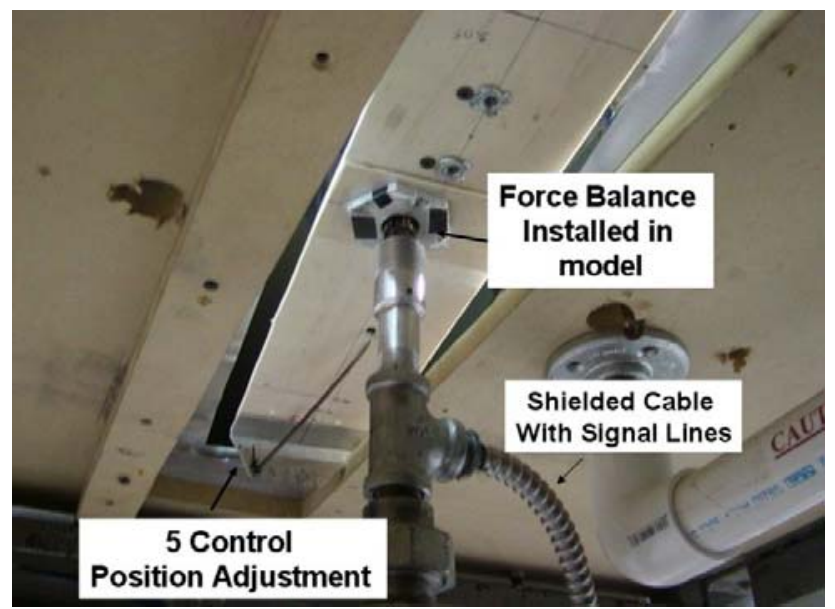

Figure 64 - Elevon equipped wing mounted on force balance at 7 degrees AOA. The force balance mounting and 5 position control adjustment can be seen clearly. 


\section{Wind Tunnel Preparation}

The West Virginia University Mechanical and Aerospace subsonic closed loop wind tunnel was constructed in 1945 and is capable of $200 \mathrm{fps}$ flows. (Figure 65) Prior to testing the wind tunnel models various flow visualization techniques were tried and work was done to restore the wind tunnels flow conditions to the best possible. During glycerin smoke flow visualization an abnormally large boundary layer was observed in the test section. Further investigation indicated that previous smoke flow visualization experiments had degraded the performance of the stainless steel screens used for flow straightening. The interior walls and screens were all thoroughly cleaned in an attempt to restore the tunnels previous published $1 \%$ turbulence intensity.
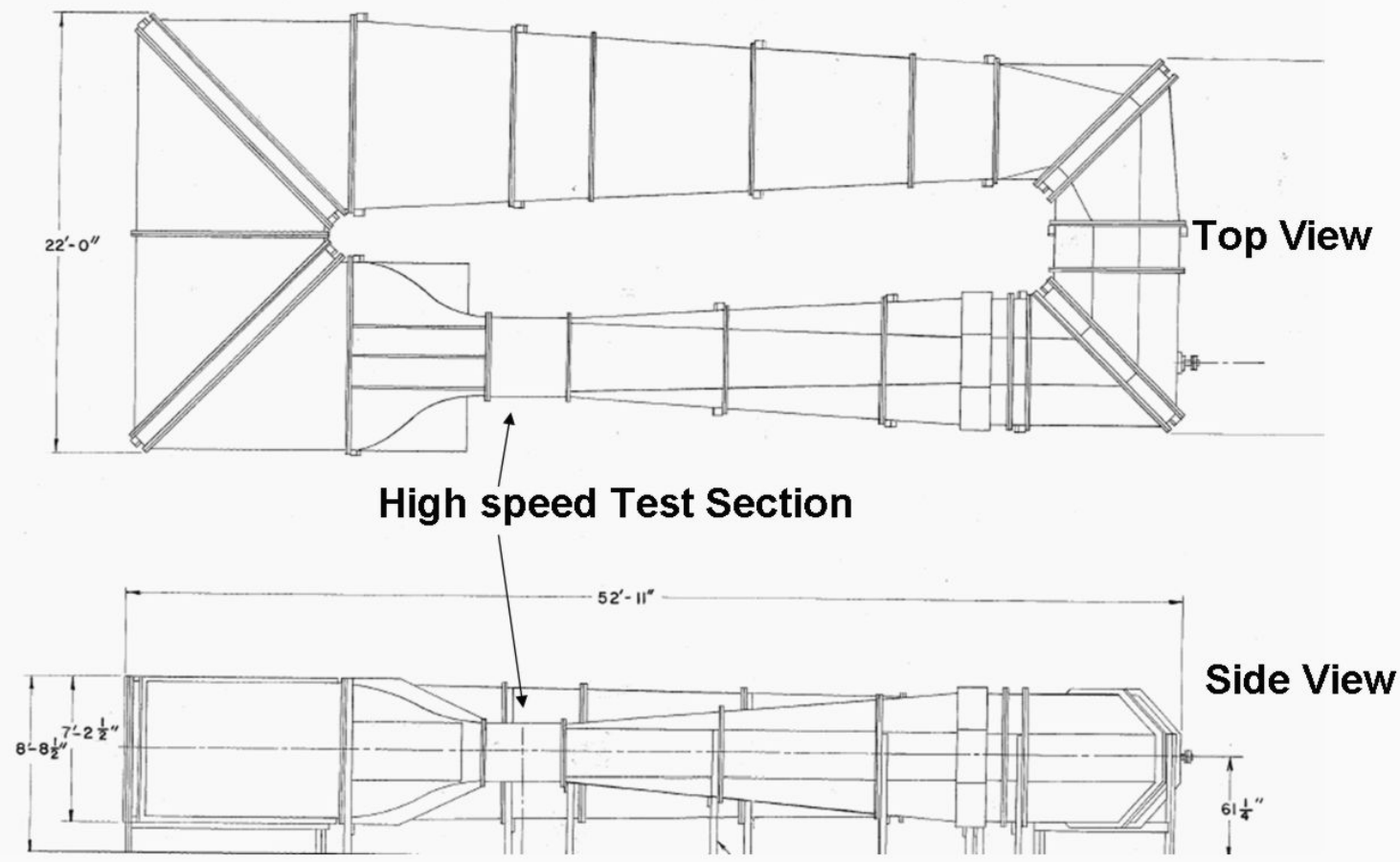

Figure 65- The West Virginia University MAE subsonic closed loop wind tunnel, constructed in 1945 and capable of 200 fps flows in good condition.

7. Data Acquisition System

The National Instruments NI-DAQ PXI-SCXI 16-bit, 8 channel data acquisition unit has been operational since November 2005. (Figure 66) Even with shielded terminal blocks, cables and aluminum BX cable electromagnetic noise presented problems from time to time. Some effects were minimized by sampling at $100 \mathrm{~Hz}$ for $1 \mathrm{sec}$ and then averaging the data for every single data point taken from the balance. There are still climbing and falling voltages at certain times which could not be cured. To ensure that these voltages 
would not affect data being acquired, test times were limited to 10 minute intervals with the system being zeroed at the beginning and then checked for zero at the end. Noise is also observed for 1 hour before any test. If noise levels are unacceptable for that time period the test will be postponed until better conditions were observed. If any channel showed a non-zero value at the end of a test sequence the data was discarded and reacquired. The NI-DAQ system uses lab view based software for control and signal sampling. This code needed to be rewritten because it used the incorrect scale factors for NASA Balance \# 826. The adherence of these basic procedures allowed a successful data acquisition program.

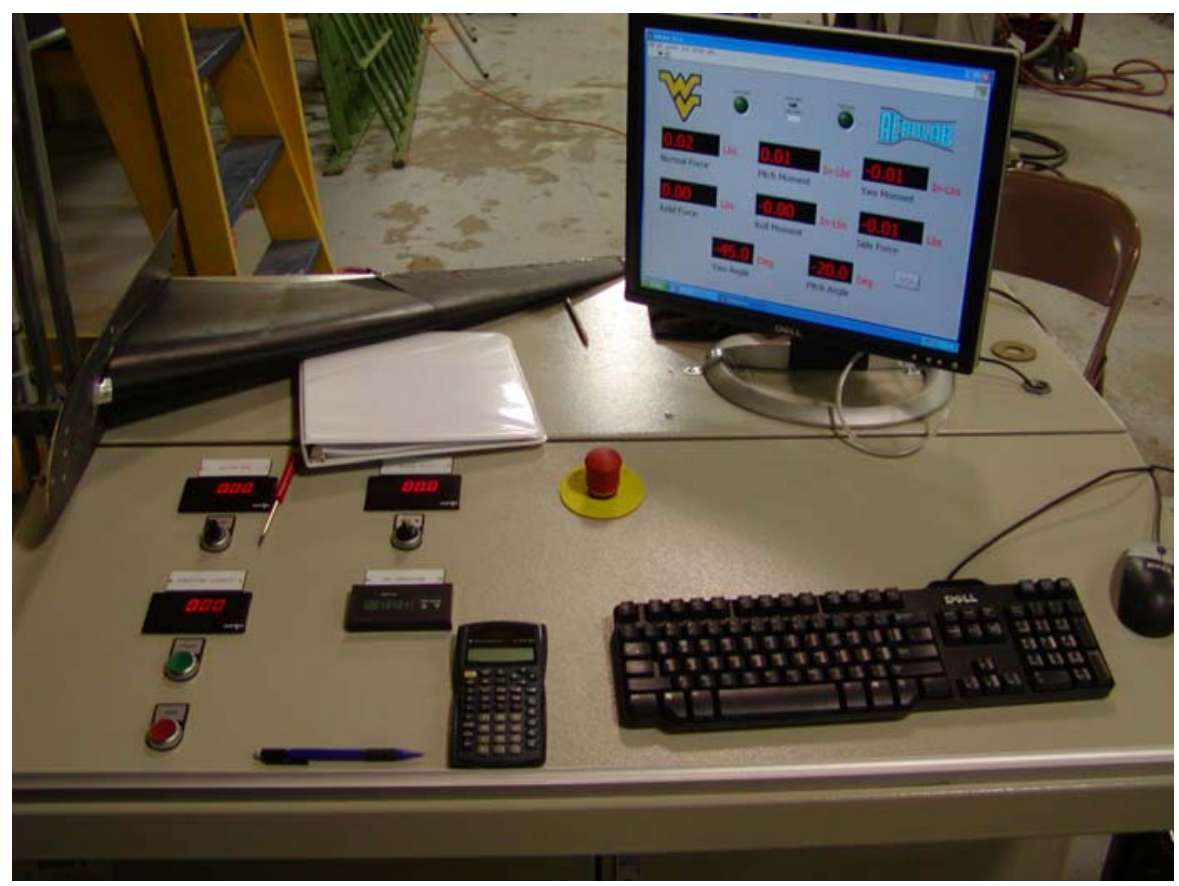

Figure 66- Data acquisition computer interface mounted on a new shielded and grounded enclosure.

\section{Force and Moment Balance System}

The 6-component balance which is on loan from NASA LRC, has been used to acquire Drag, Pitch, Yaw and Roll data. (Figure 67) Repairs made by the balance by it's original manufacturer $M$ and M Machine Tool in Norfolk Virginia required a new calibration because of changes in the axial strain gauge bridge. 


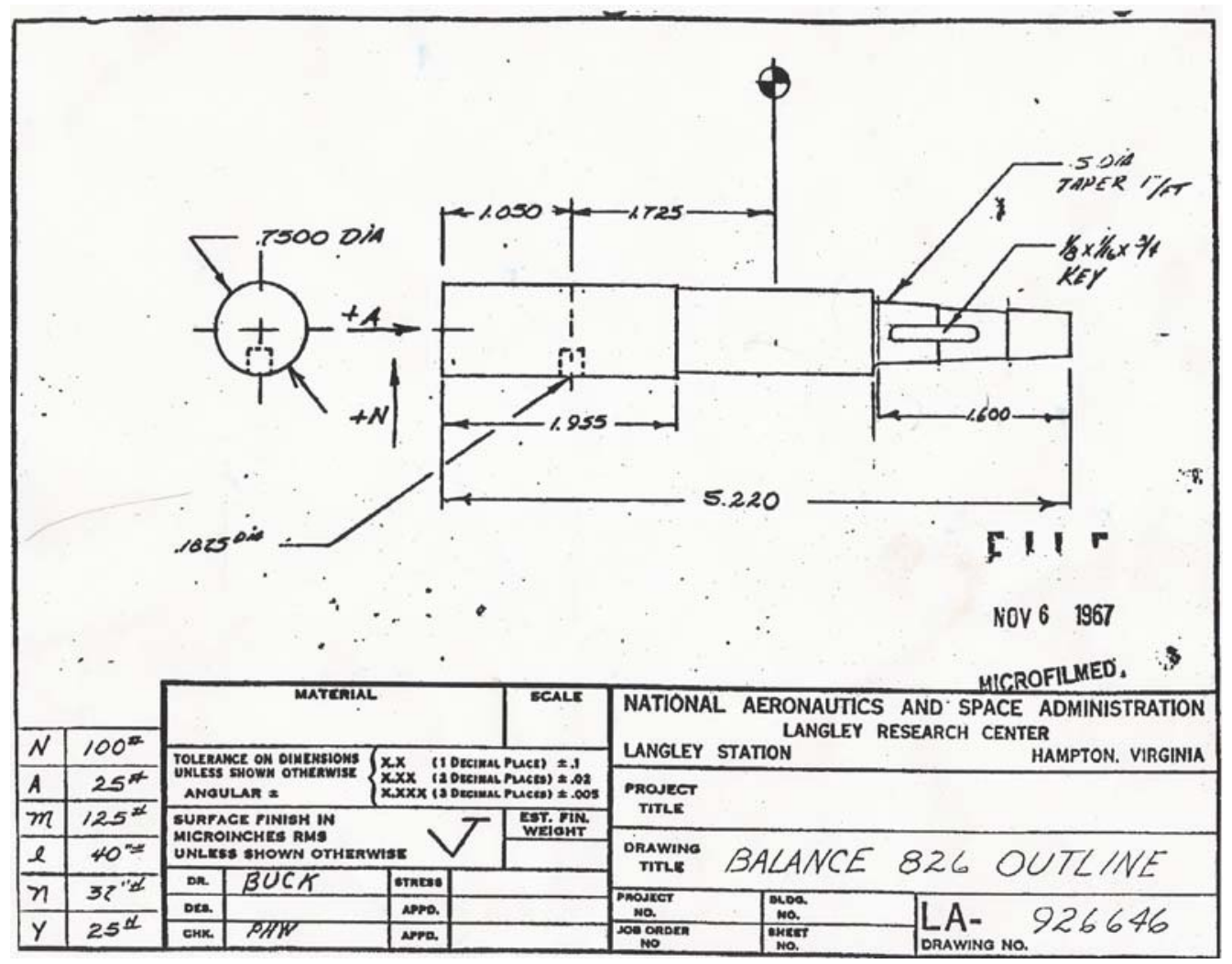

Figure 67 - Schematic of NASA Force Balance \#826.

Initial calibration attempts proved to be difficult because NASA Balance \# 826 was designed as a sting balance, but for the half span models used in this project is being used in the way a pyramidal balance would be used. The initial design for the model mount had the balance in it's intended orientation just under the test section floor. After the difficulties in properly accounting for the balance interactions it was decided to redesign the model mount. The relatively large load capabilities of Balance \#826 allowed for the orientation of the balance to be changed from horizontal to vertical. Table 3 shows the change in axis for the force balance in the WVU wind tunnel orientation. The end of the balance is now inserted vertically into the wing model at the desired center of gravity. The third generation mount was designed so that the moment center of balance would correspond with root of the test models and at the desired Cg location.

Table 3 - Force Balance Axis Change for WVU test.

\begin{tabular}{|c|c|}
$\begin{array}{c}\text { WVU Test } \\
\text { Orientation }\end{array}$ & $\begin{array}{c}\text { Balance } \\
\text { Design } \\
\text { Orientation }\end{array}$ \\
\hline Vertical & Axial \\
\hline Lift & Side \\
\hline Drag & Normal \\
\hline Roll & Yaw \\
\hline Pitch & Roll \\
\hline
\end{tabular}


Each channel on the force balance was then calibrated using weights and pulleys to generate pure forces and moments. (Figure 68) Then the interactions between the altered strain gauge bridge and the remaining were calculated. When everything is setup properly, the known loads or moments have a slope of 1 when plotted against the corresponding reading from the data acquisition system. Figure 68 shows the lift load interaction curves with the Lift load - Lift reading slope and the Lift load - Axial reading slopes shown. All six load and moment calibration curves are included in Appendix A and the interaction matrices are included in Figure 69. The slope of this curve is known as the interaction coefficient. A similar curve was developed for each known load and the six corresponding readings from the six components of the force balance.

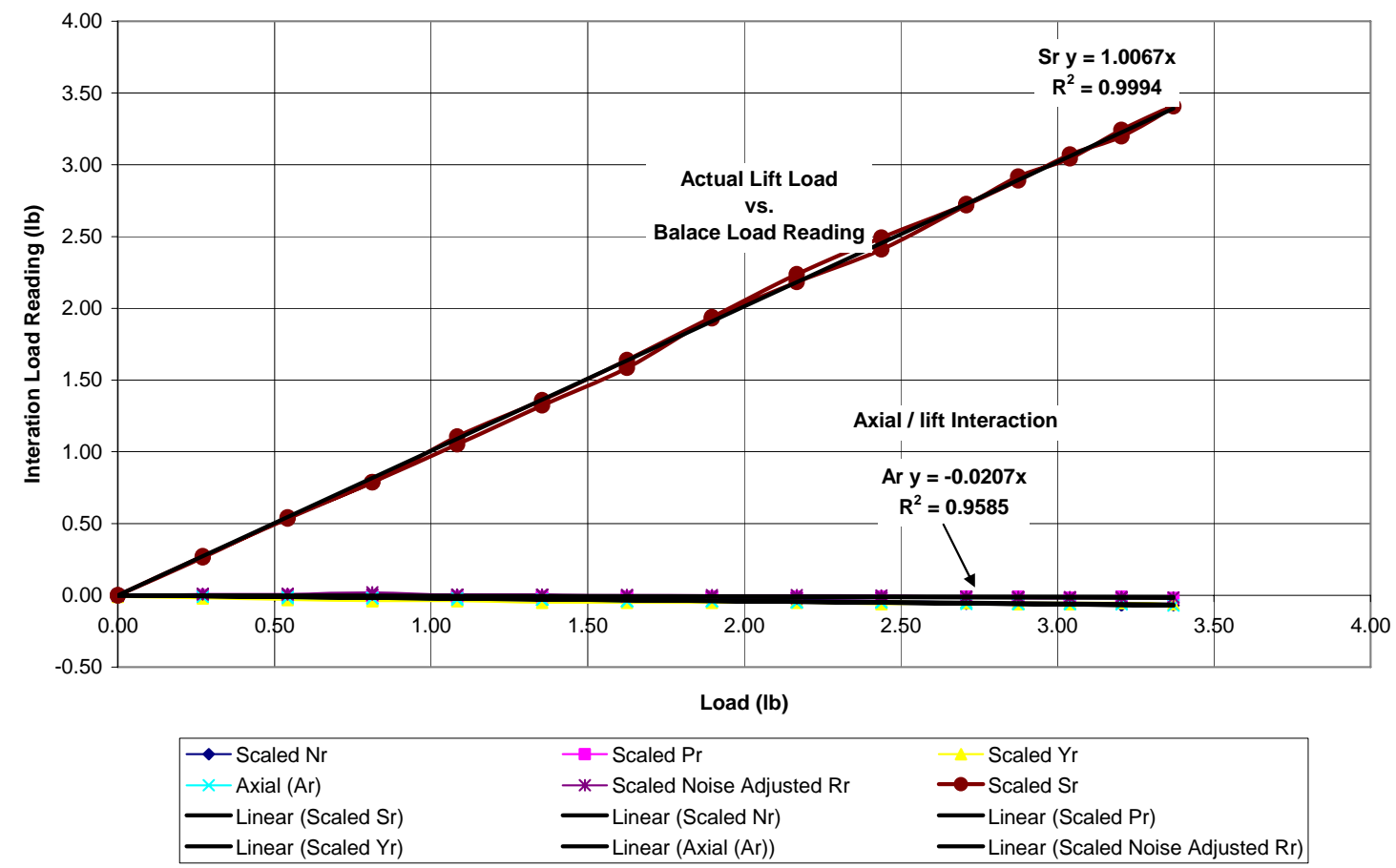

Figure 68 - Example of the calibration curves for a known lift load for the calculation of interaction coefficients.

Because only the interactions between the known loads and the axial channel had changed the new Axial interaction coefficients were calculated and inserted into the original NASA interaction coefficient matrix. The interaction matrix then allows the calculation of wind-tunnel loads from the six component balance. Each load component can be seen as a linear combination of six interacting functions. The linear approximation relating loads to reading is as follows: 


$$
\begin{array}{lll}
\mathrm{Fr}=[\mathrm{Kij}]^{*}(\mathrm{Fl}) & & \text { Equation 1 } \\
\mathrm{Fl}=[\mathrm{Kij}]^{-1 *}(\mathrm{Fr}) & \text { or } & \\
& & \text { Equation 2 }
\end{array}
$$

Here $\mathrm{Fl}$ is made up of the six load components, $\mathrm{Fr}$ is made up of the six readings from the balance, [Kij] is the $6 \times 6$ interaction matrix made up of slopes from the interaction

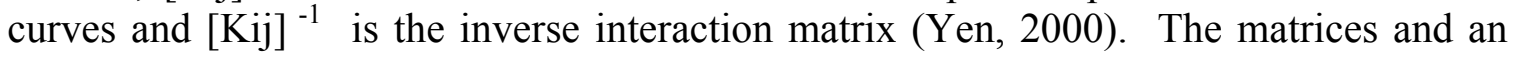
example calculation is shown in Figure 69- Six Component interaction load calculation work sheet for NASA balance interaction matrix as setup in WVU wind tunnel. Light green cells show new axial interaction coefficients.

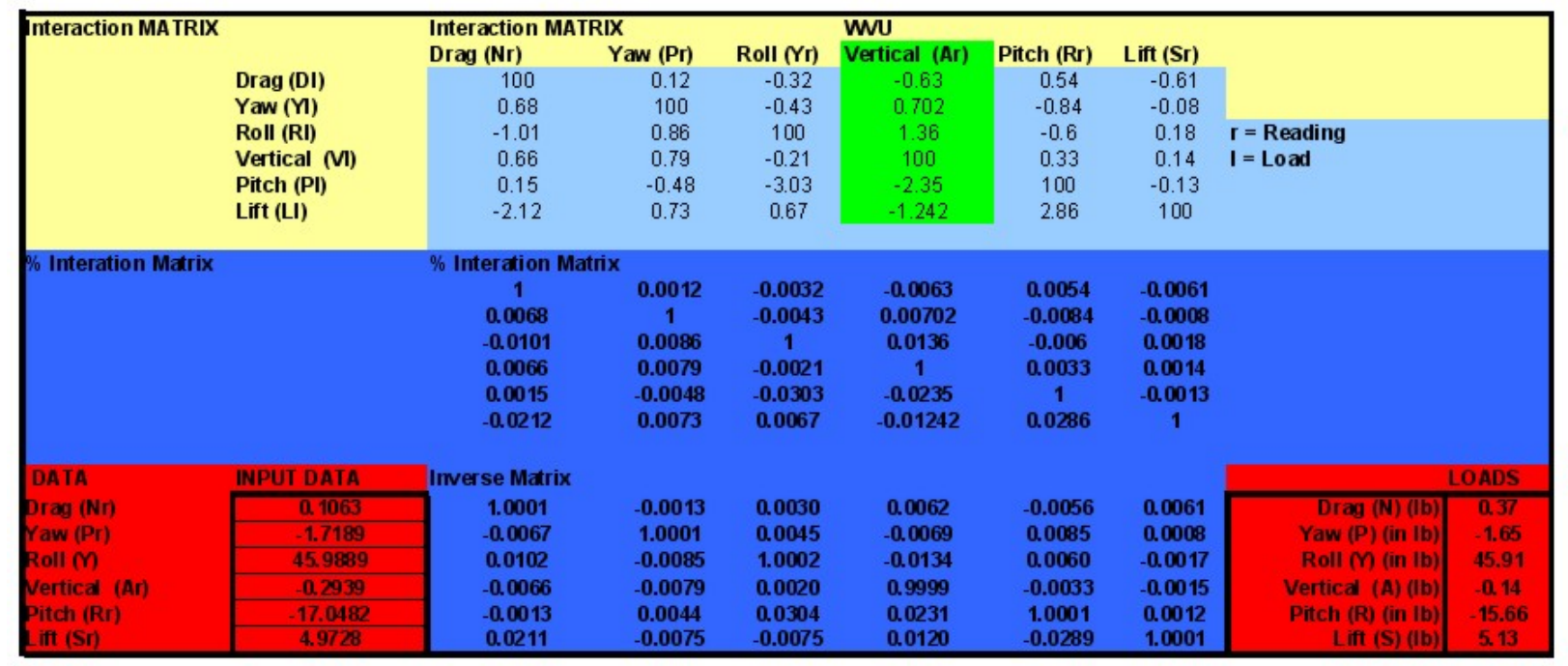

Figure 69- Six Component interaction load calculation work sheet for NASA balance interaction matrix as setup in WVU wind tunnel. Light green cells show new axial interaction coefficients.

\section{Wind Tunnel Test Case}

In order to validate the procedures and experimental setup of the WVU wind tunnel it was desirable to conduct a validation study where a good quality published data set is matched in the WVU tunnel. A variety of simple 2D and 3D wind tunnel studies were identified in order to provide a validation study for the wind tunnel aerodynamics and the force balance combination. The majority of these studies had test conditions that were too far removed from the type of wind-tunnel studies done in this project or they had conditions which could not be duplicated in the WVU wind tunnel, such as too high a Reynolds number. The WVU wind tunnel has a current velocity limitation of approximately $50 \mathrm{~m} / \mathrm{s}$. The study which was chosen for a test case is a study conducted by Gerontakos and Lee in 2006 which was published in Experiments in Fluids. Their study is titled "Nearfield tip vortex behind a swept wing model" and provided tip and wake vorticy data as well as coefficient of lift and drag data for a swept NACA 0015 wing and a NACA 0015 wing with square tips and an aspect ratio of 2.5. (Figure 70) 


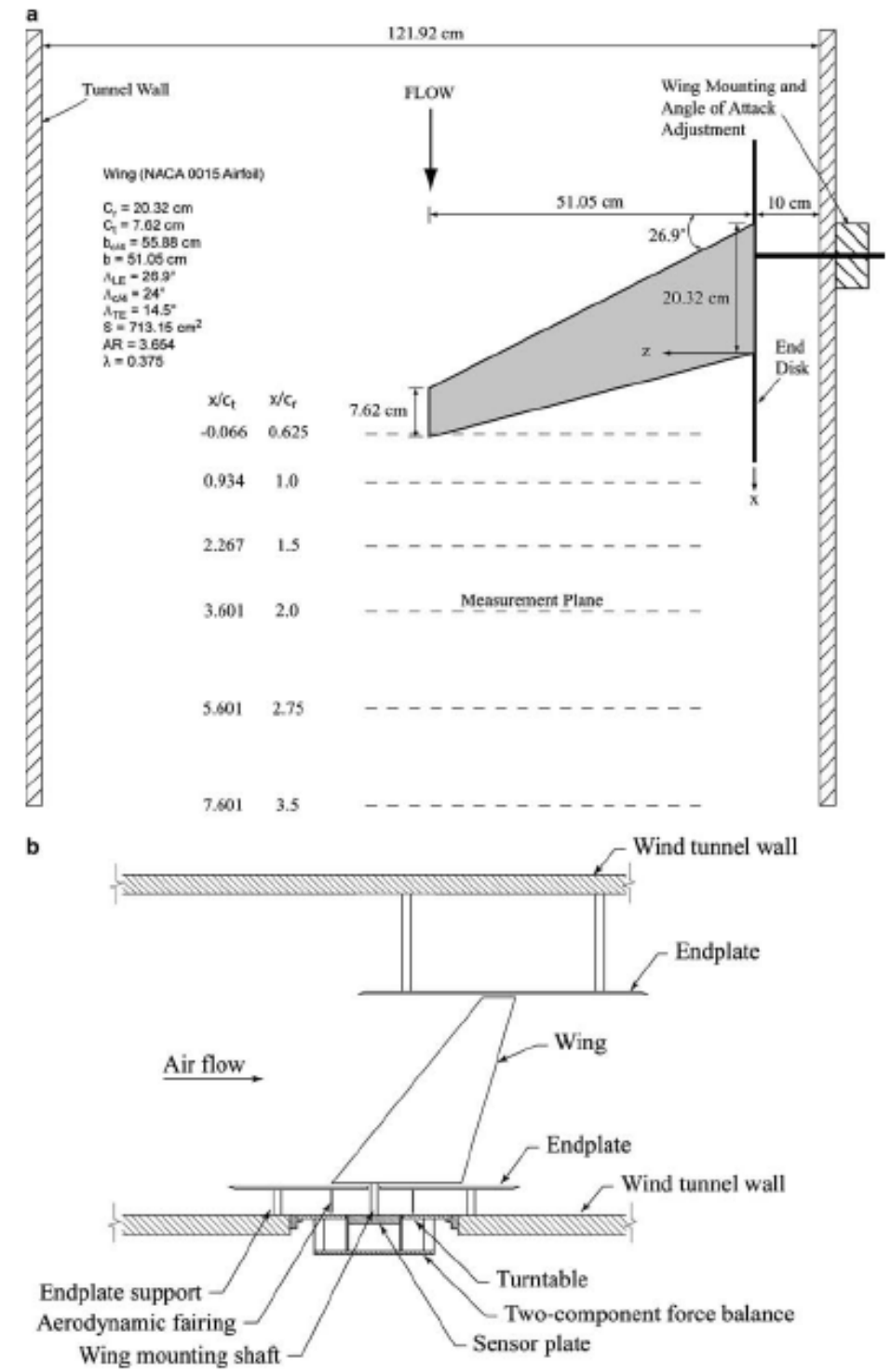

Figure 70- Gerontakos and Lee's experimental setup to study tip vortices and then to remove tip effects from the same wing.

Lift and drag data was generated from both two component force balance data as well as surface and wake pressure data. The experiment was carried out in the recently constructed low-turbulence $0.9 \times 1.2 \times 2.7$ m suction-type subsonic wind tunnel at McGill University with a freestream turbulence intensity of $0.03 \%$ at $35 \mathrm{~m} / \mathrm{s}$. All studies were carried out at a Reynolds number of 181,000. Gerontakos and Lee constructed both swept wing and rectangular wing models in order to study the tip vortices. In this study a flat plate mounted to the tunnel wall was brought in to remove the tip effects. Two sets of data were collected for each wing. One set of data is similar to a 2D data set and the other without the tip plate is 3D. The data lift and drag data collected from these studies is included in Figure 71 \& Figure 72. 


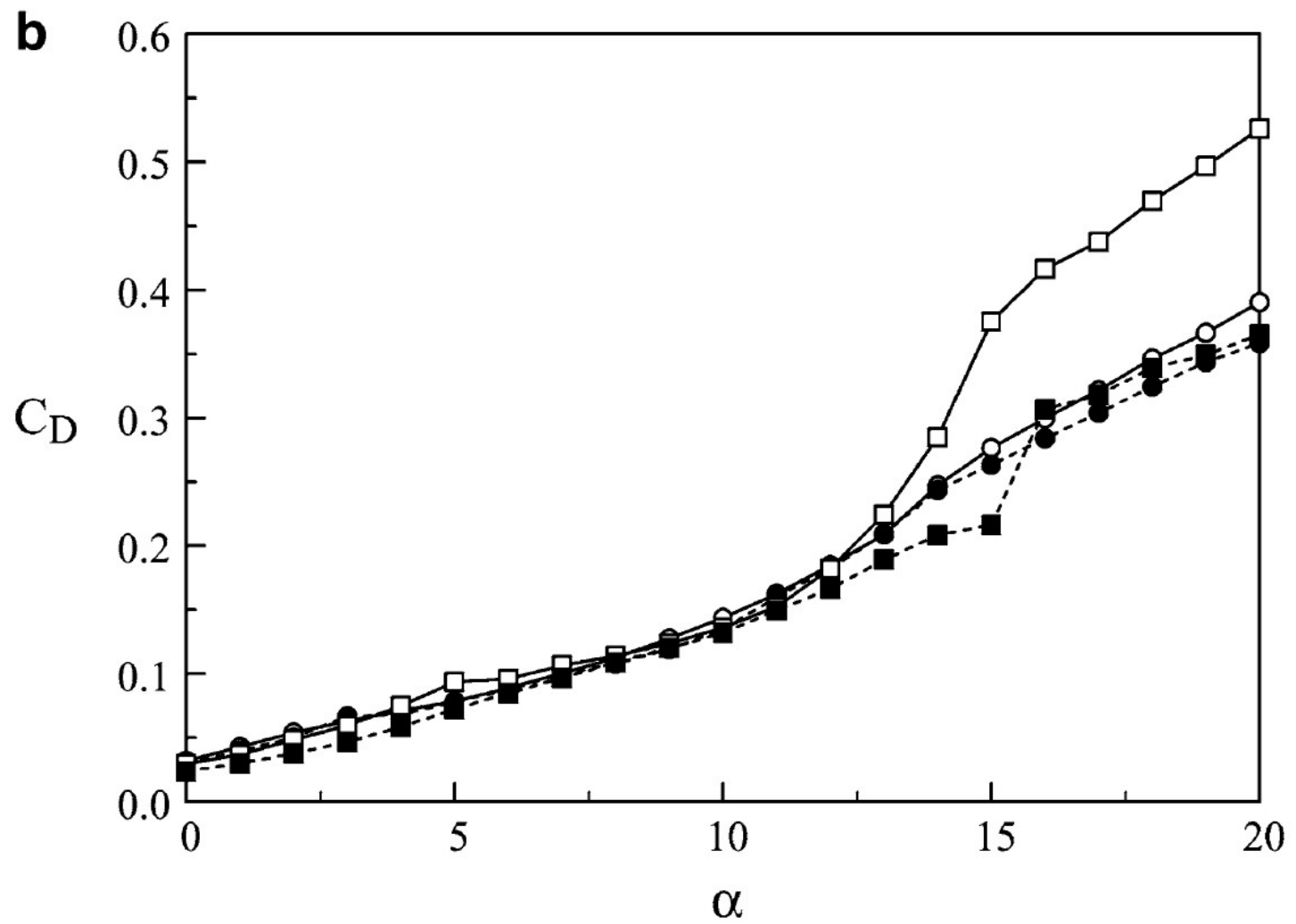

Figure 71- Coefficient of drag data from the 2006 Gerontakos and Lee study.

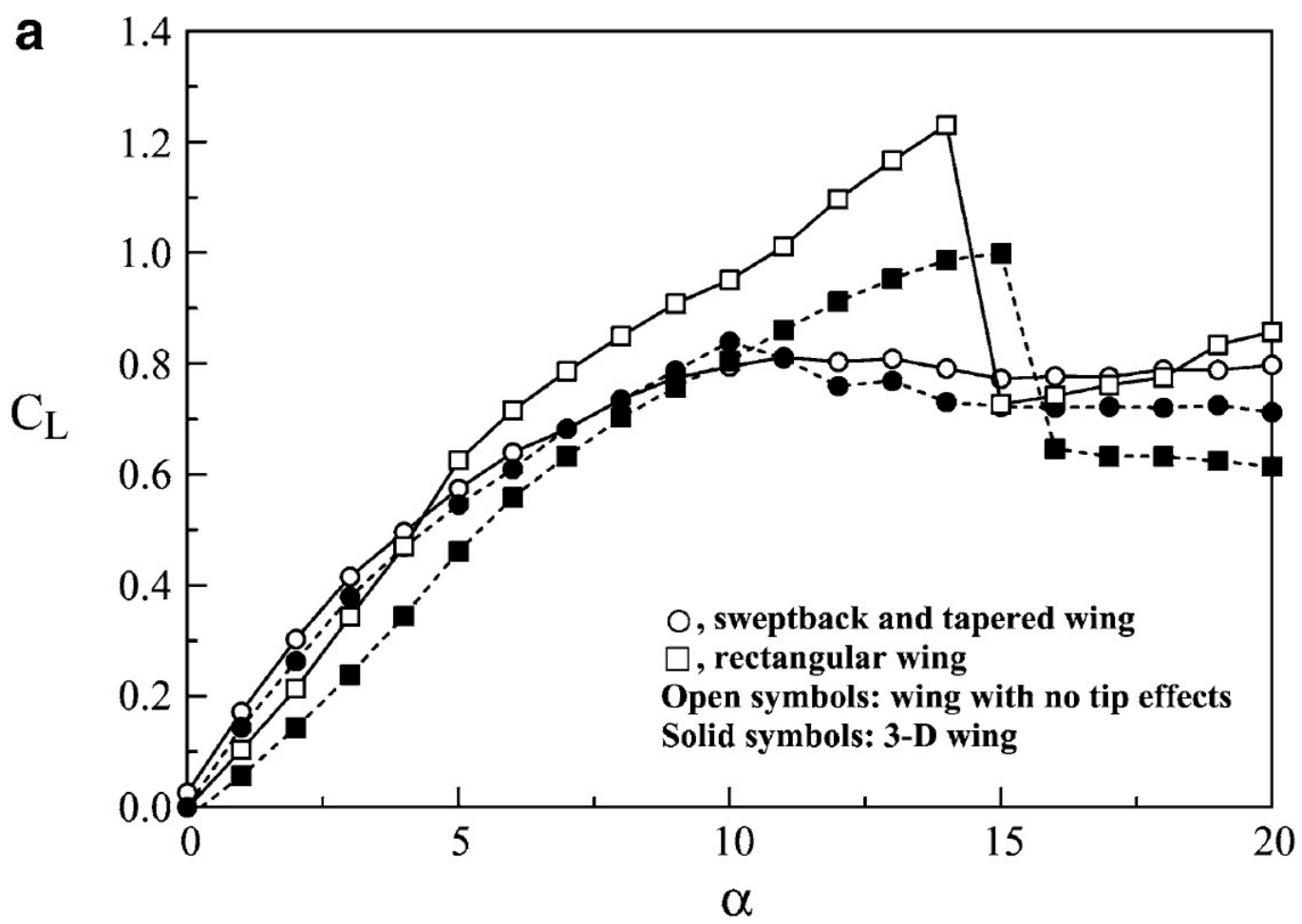

C

$\mathrm{C}_{\mathrm{L}}$

Figure 72 - Coefficient of lift data from the 2006 Gerontakos and Lee study. 
For this wind tunnel test study a fiberglass, plywood and aluminum model of aspect ratio 2.5 was constructed with a cord of 11" and a half span of 14". (Figure 73 \& Figure 74) The size of the model was determined in order to obtain an aspect ratio of 2.5 and a thickness great enough for the insertion of a force balance at the $1 / 4$ cord. The WVU NACA 0015 model has a square tip geometry and was studied at various AOAs at a Reynolds number of $1.83 \times 10^{5}$. Force and moment data was collected using the NASA six component balance \#826. Data collected for the WVU tunnel is compared to the data in the Experiments in Fluid paper in Figure 75 and Figure 76.

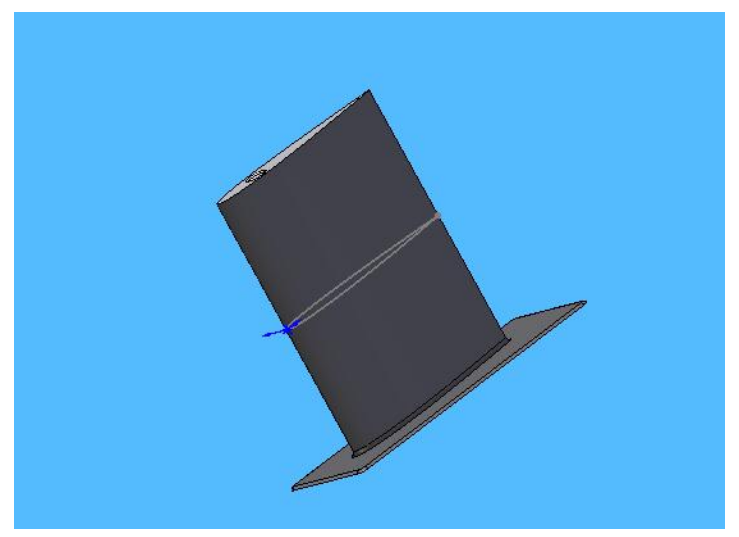

Figure 73 - Validation Wind tunnel Model, NACA 0015, AR-2.5, 11"cord for Re=181000.

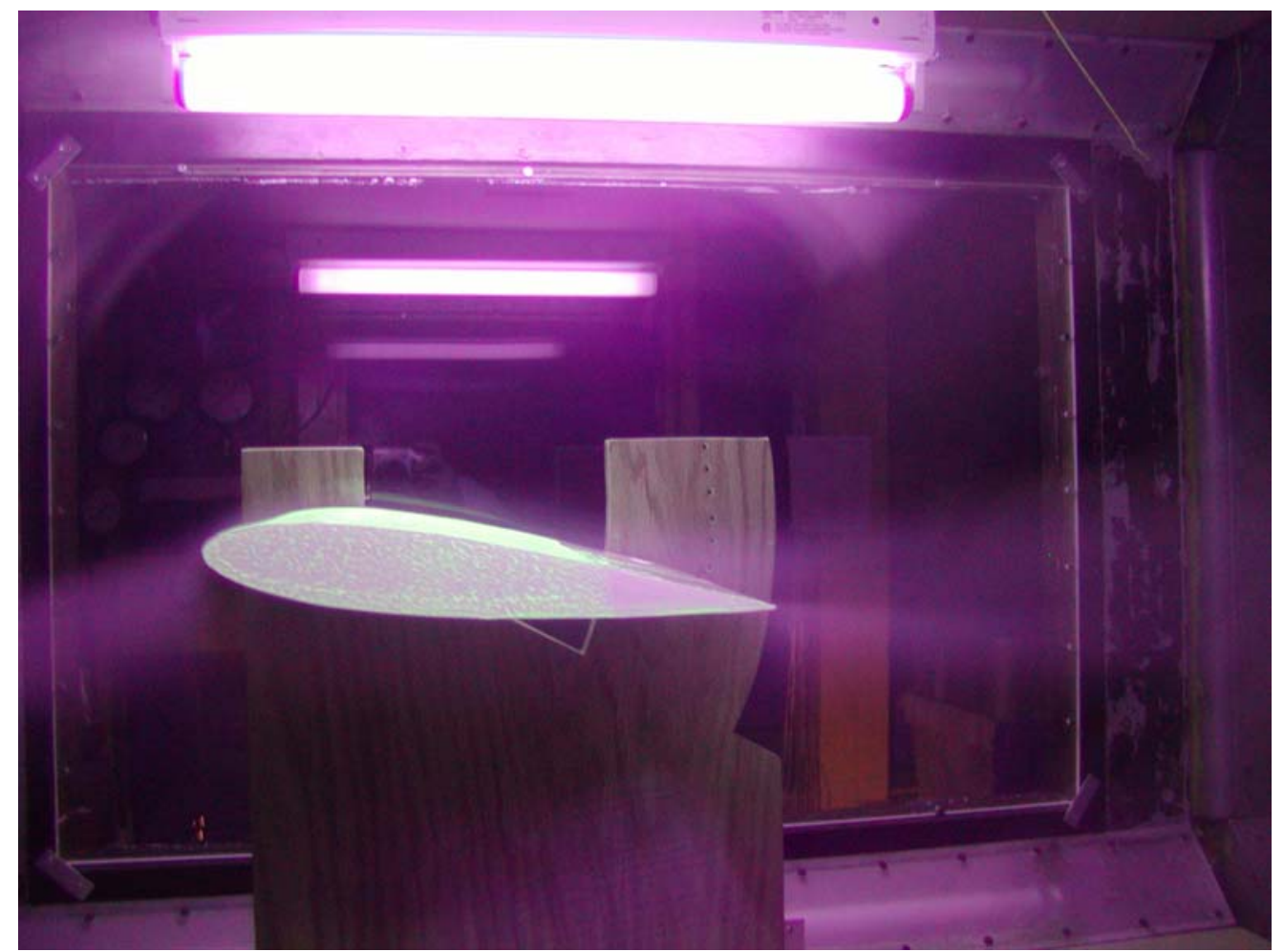

Figure 74 - WVU NACA 0015 validation model in the horizontal variable AOA mount during smoke flow visualization. 


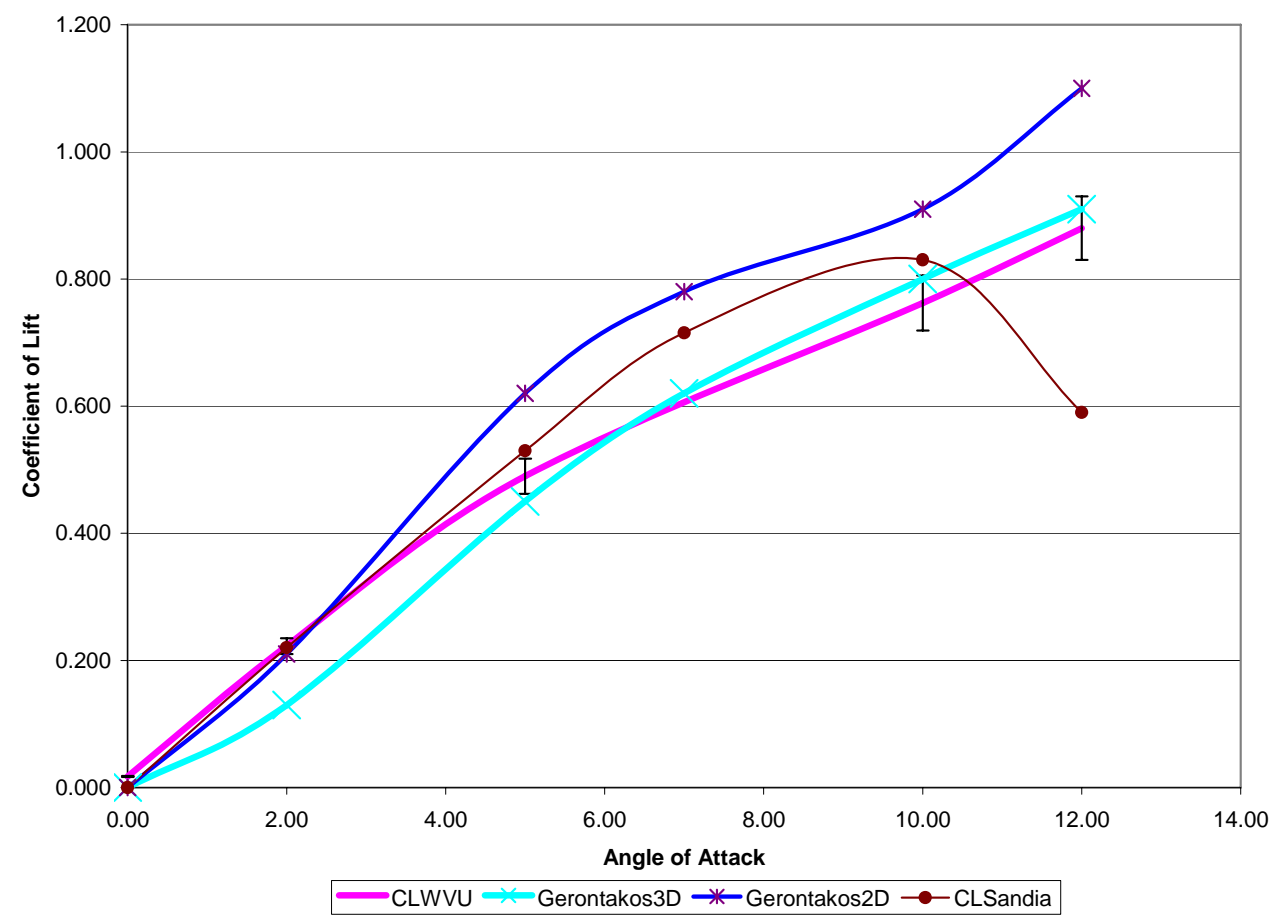

Figure 75- Coefficient of lift data comparison between WVU NACA 0015 validation model data, Gerontakos data and 2D Re 160000 data from Sandia National Labs.

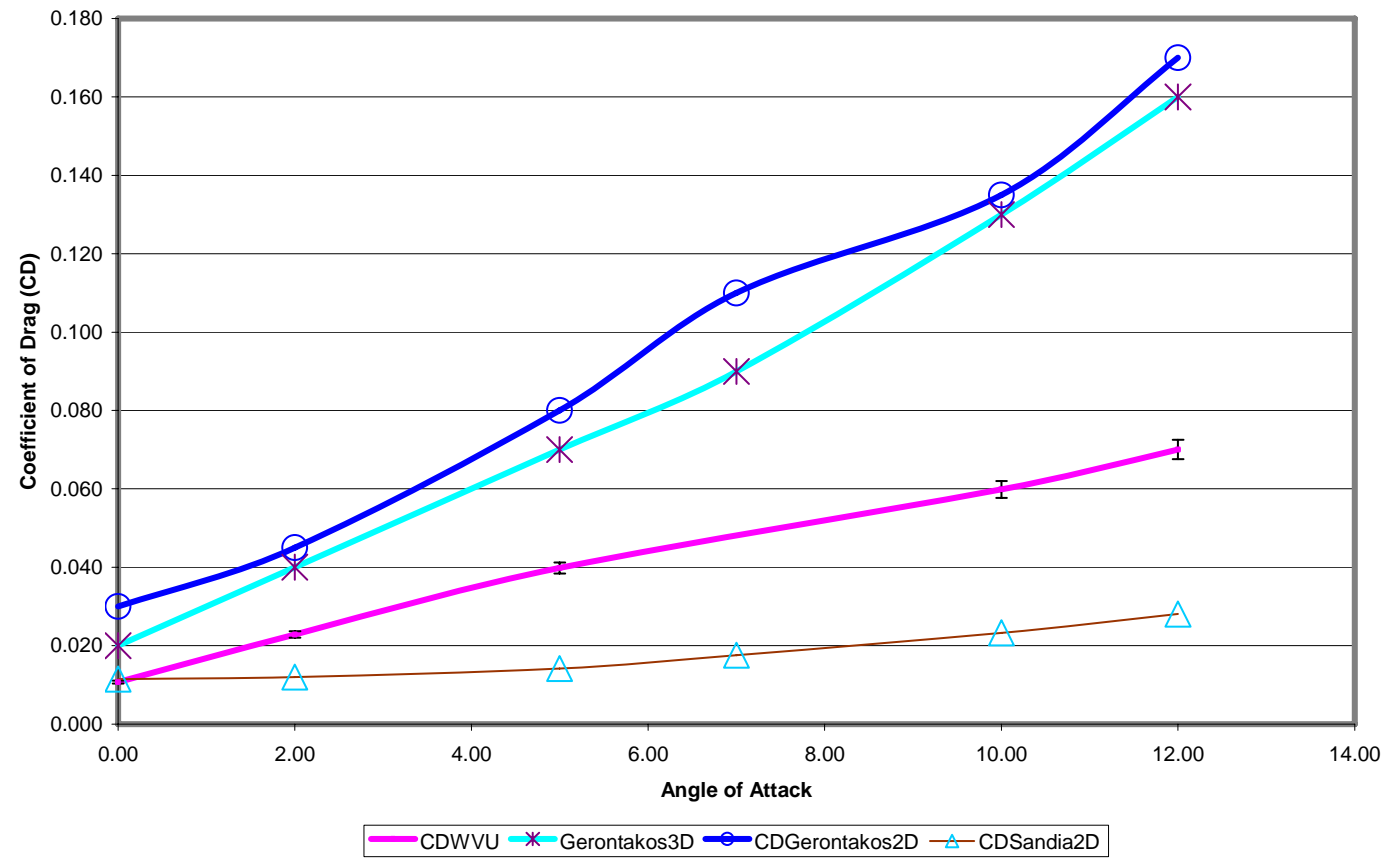

Figure 76- Coefficient of drag data comparison between WVU NACA 0015 validation model data, Gerontakos data and 2D Re 160000 data from Sandia National Labs. 
There is good agreement between coefficient of lift data collected at WVU and that collected by the 2006 Gerontakos and Lee study. There is a small Reynolds number difference between the two data sets. The WVU data set was collected at 183,000 and the Gerontakos data was collected at 181,000 . This normally would be insignificant, but from previous tests at WVU this Reynolds number range has shown there to be large variations in lift and drag values with small variations of Re.

Drag coefficient data agreement between the WVU and Gerontakos and Lee data set was not as good, but the WVU data is bracketed in between 2D NACA 0015 data published from Sandia Labs and Gerontakos's 3D data. The WVU drag at zero angle of attack is only slightly higher at $\mathrm{C}_{\mathrm{D}}=0.012$ then Sandia's $2 \mathrm{D}$ zero degree AOA which is reasonable were as the Gerontakos drag coefficient at zero degree AOA is over twice as high as Sandia's 2D data. This may indicate that experimental setup such as model mounting or pressure tap plumbing in Gerontakos's experiments may be contributing to the drag of the model. Overall the WVU data is satisfactory for the wind tunnel comparisons between the Horten type swept wing model and the morphing wing model.

\section{Wind Tunnel Testing}

Initial wind tunnel data was collected in the spring of 2005 at the Vigyan wind tunnel in Hampton, Virginia. The 2005 wind tunnel testing was marred by errors which came from ballooning of the latex skin of the morphing wing and the warping of the plywood ground plates due to high humidity of the time. Due to expense the scope of the Vigyan testing was rather small. Data at the five control positions was collected at one velocity and at 0 degrees AOA.

During the spring and summer of 2006 wind tunnel testing collected basic forces and Moments for both the elevon equipped model and the morphing model. Both were tested at $32 \mathrm{ft} / \mathrm{sec}(10 \mathrm{~m} / \mathrm{s}), 49 \mathrm{ft} / \mathrm{sec}(15 \mathrm{~m} / \mathrm{s})$ and $68 \mathrm{ft} / \mathrm{sec}(21 \mathrm{~m} / \mathrm{s})$ and $0,3.5$ and $7 \mathrm{degree}$ angle of attack (AOA) in the $32 \times 45$ inch test section on the WVU wind tunnel.

The three test velocities Reynolds numbers ranged between 186,000 and 375,000. Test velocities were calculated using the wind-tunnel head, barometric pressure and air temperature. Figure 77 shows an example test condition spread sheet. 


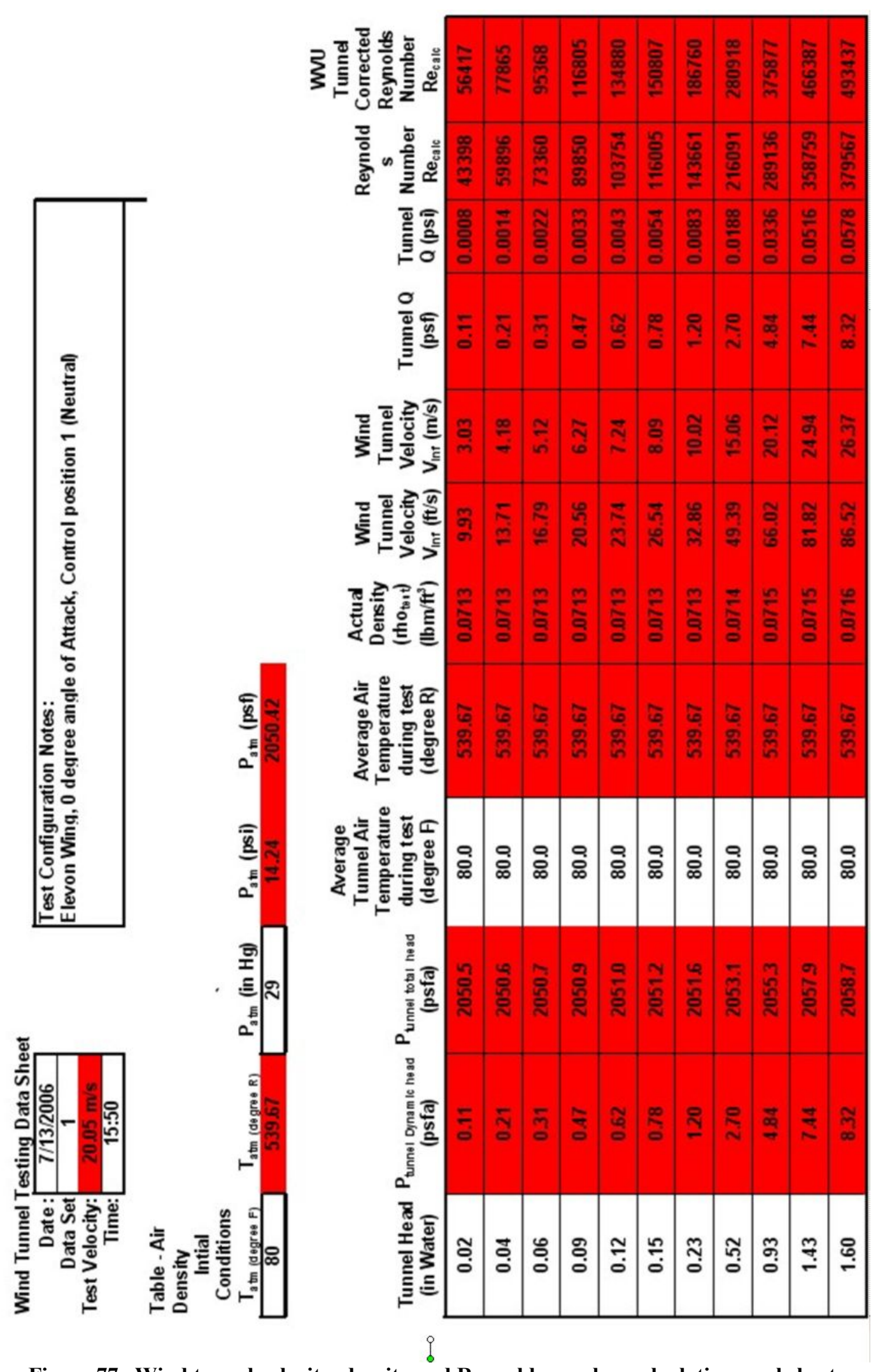

Figure 77- Wind tunnel velocity, density and Reynolds number calculation worksheet 


\section{Basic Control Force and Moment Study}

The average of three sets of six raw load and moment data points were collected from the force balance for each test. These numbers which correspond to Lift, Drag, Pitch Moment, Roll Moment and Yaw Moment were adjusted for any balance related interactions. The forces and moments were then converted to nondimensional coefficients for comparison. Lift $\left(\mathrm{C}_{\mathrm{L}}\right)$ and Drag $\left(\mathrm{C}_{\mathrm{D}}\right)$ coefficients were nondimensionalized using the wing area of 1.317 sq. feet. Pitching Moment $\left(\mathrm{C}_{\mathrm{mP}}\right)$ was nondimensionalized using the wing area times the mean aerodynamic cord (MAC) of $0.731 \mathrm{ft}$., Roll $\left(\mathrm{C}_{\mathrm{mR}}\right)$ and Yaw $\left(\mathrm{C}_{\mathrm{mY}}\right)$ were nondimensionalized using the wing area times the model span of $2.0 \mathrm{ft}$. The calculated aerodynamic coefficients for both wings at 7 degrees AOA and $20 \mathrm{~m} / \mathrm{s}$ are shown below. All experimental aerodynamic coefficients from 2006 and 2007 are included in Appendix B.

Table 4- Experimental aerodynamic coefficients for the elevon equipped wing at 7 degrees AOA and $21 \mathrm{~m} / \mathrm{s}$.

\begin{tabular}{|c|c|c|c|c|c|c|}
\hline Control Position & $C_{D}$ & $C_{m Y}$ & $\mathrm{C}_{\mathrm{mP}}$ & $\mathrm{C}_{\mathrm{mR}}$ & $C_{L}$ & $\begin{array}{l}\text { L/D } \\
\text { elevon }\end{array}$ \\
\hline 7 & 0.055 & 0.001 & -0.099 & 0.122 & 0.376 & 8.33 \\
\hline 3.5 & 0.055 & 0.002 & -0.061 & 0.096 & 0.326 & 7.79 \\
\hline 0 & 0.057 & 0.002 & -0.033 & 0.077 & 0.289 & 5.09 \\
\hline-3.5 & 0.053 & 0.000 & -0.130 & 0.143 & 0.410 & 7.79 \\
\hline-7 & 0.053 & -0.002 & -0.158 & 0.162 & 0.444 & 8.33 \\
\hline
\end{tabular}

Table 5- Experimental aerodynamic coefficients for the Morphing wing at 7 degrees $\mathrm{AOA}$ and 21 $\mathbf{m} / \mathbf{s}$.

\begin{tabular}{|c|c|c|c|c|c|c|}
\hline $\begin{array}{l}\text { Control } \\
\text { Position }\end{array}$ & $C_{D}$ & $\mathrm{C}_{\mathrm{mY}}$ & $\mathrm{C}_{\mathrm{mP}}$ & $C_{m R}$ & $C_{L}$ & $\begin{array}{c}\text { L/D } \\
\text { morphing }\end{array}$ \\
\hline-7 & 0.036 & 0.001 & -0.106 & 0.138 & 0.418 & 11.49 \\
\hline-3.5 & 0.039 & 0.001 & -0.082 & 0.120 & 0.386 & 9.97 \\
\hline 0 & 0.041 & 0.000 & -0.055 & 0.101 & 0.351 & 8.60 \\
\hline 3.5 & 0.036 & 0.000 & -0.126 & 0.153 & 0.439 & 12.36 \\
\hline 7 & 0.033 & -0.004 & -0.165 & 0.182 & 0.497 & 14.94 \\
\hline
\end{tabular}

The force and moment behavior between the elevon and morphing wings in their Spring 2006 configuration are very similar. The morphing wing displays slightly higher $\mathrm{C}_{\mathrm{L}} \mathrm{S}$ and lower $\mathrm{C}_{\mathrm{D}}$ s. Force and moment data is presented in Table 4 and Table 5 and graphically in Figure 78 and Figure 79. 


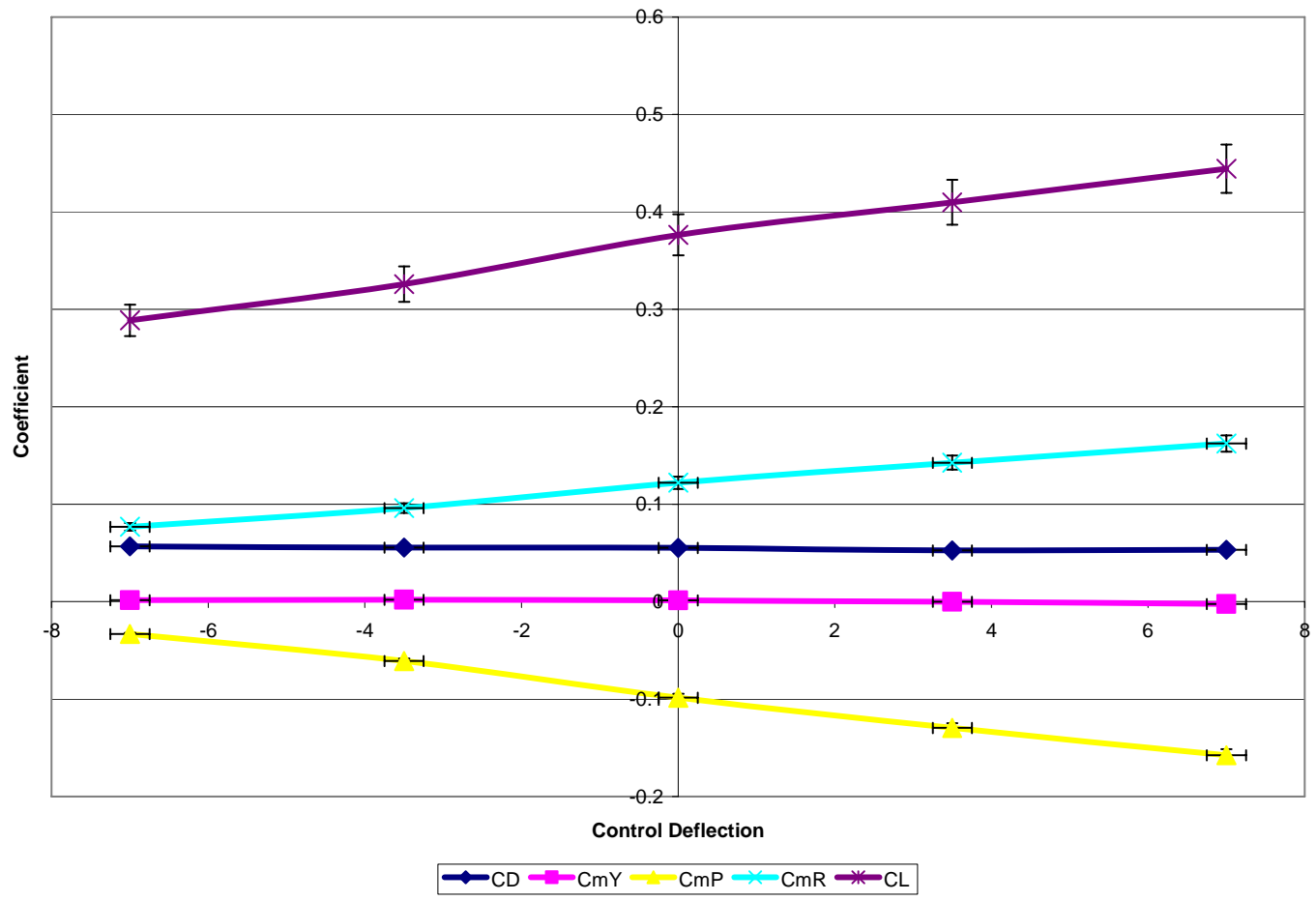

Figure 78- Experimental aerodynamic coefficients for the elevon equipped wing at 7 degrees AOA and $68 \mathrm{ft} / \mathrm{sec}(21 \mathrm{~m} / \mathrm{s})$.

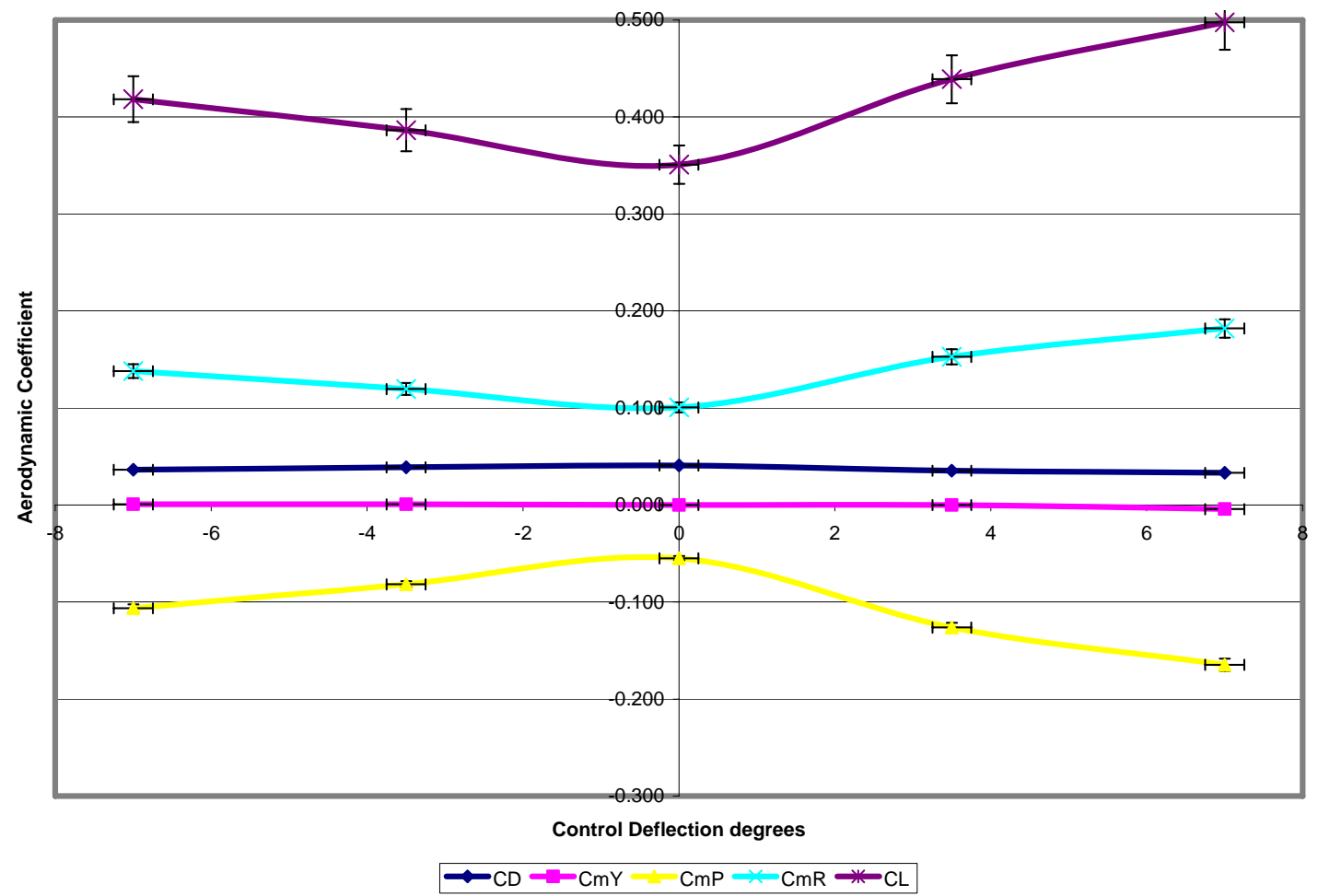

Figure 79- Experimental aerodynamic coefficients for the orphing wing at 7 degrees $\mathrm{AOA}$ and 68 $\mathrm{ft} / \mathrm{sec}(21 \mathrm{~m} / \mathrm{s})$. 
Figure 80 compares the $C_{L}$ and $C_{D}$ s of both wings at 0 degrees angle of attack and $21 \mathrm{~m} / \mathrm{s}$ and Figure 81 compares the $C_{L}$ and $C_{D}$ both wings at 7 degrees angle of attack and 21 $\mathrm{m} / \mathrm{s}$. The behavior of these curves changes dramatically as lift increases, either with increased angle of attack or increased tunnel velocity. Data was acquired at 10, 15and 21 $\mathrm{m} / \mathrm{s}$, but the basic control behavior of these wings appears to change between 15 and 21 $\mathrm{m} / \mathrm{s}$ or between Reynolds numbers of 280,000 and 370,000. The actual performance of these wings can be seen more clearly by comparing the Lift over Drag ratios at the various control positions as can be seen in Figure 82. Even though the wings were mounted parallel to the centerline of the test section, the negative $\mathrm{C}_{\mathrm{L}}$ at 0 degree AOA for the morphing wing may indicate that the actual orientation of the wings to the flow give a slight negative AOA.

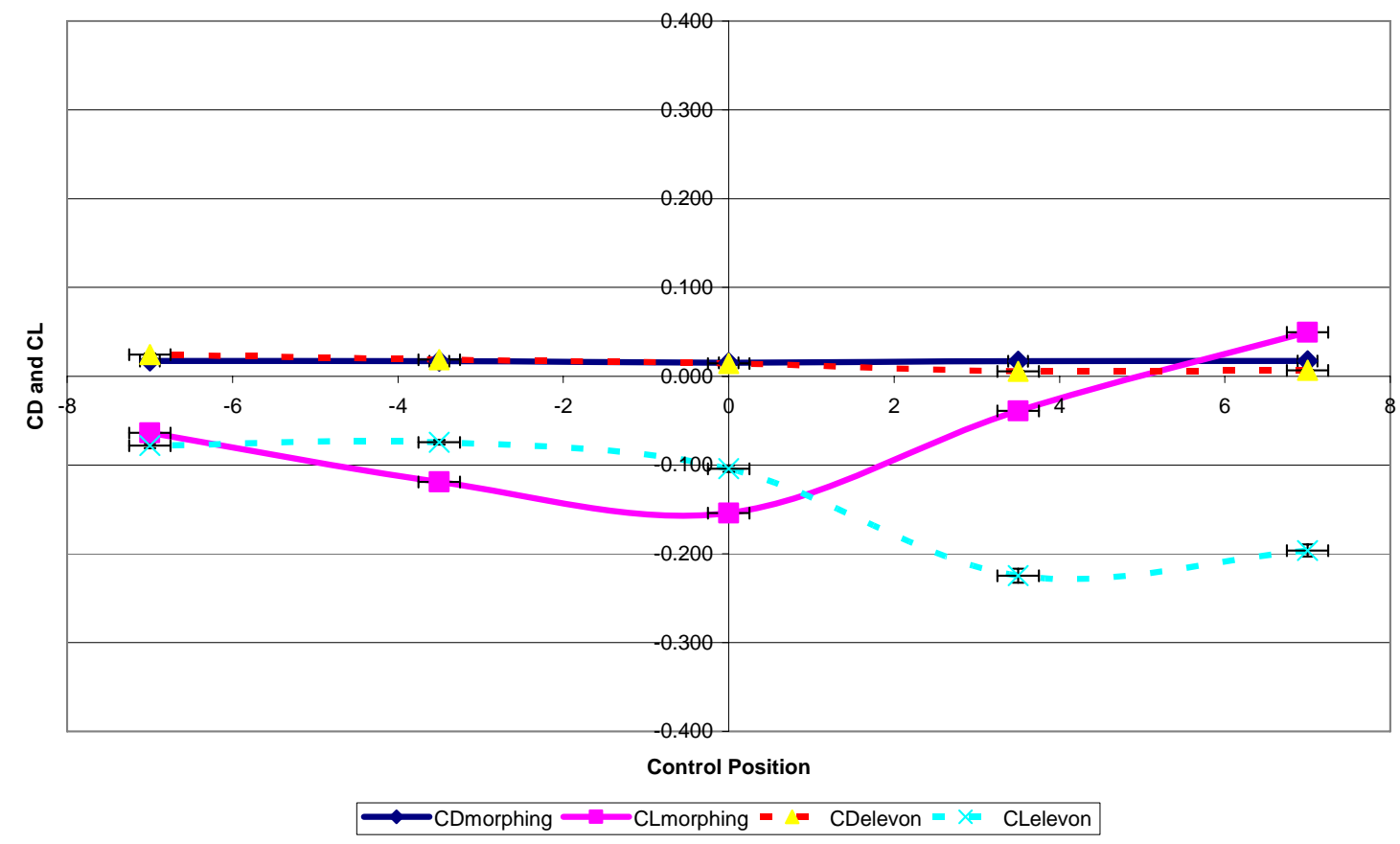

Figure 80- Experimental $C_{L}$ and $C_{D}$ aerodynamic coefficients for both wings at 0 degrees $A O A$ and $68 \mathrm{ft} / \mathrm{sec}(21 \mathrm{~m} / \mathrm{s})$. 


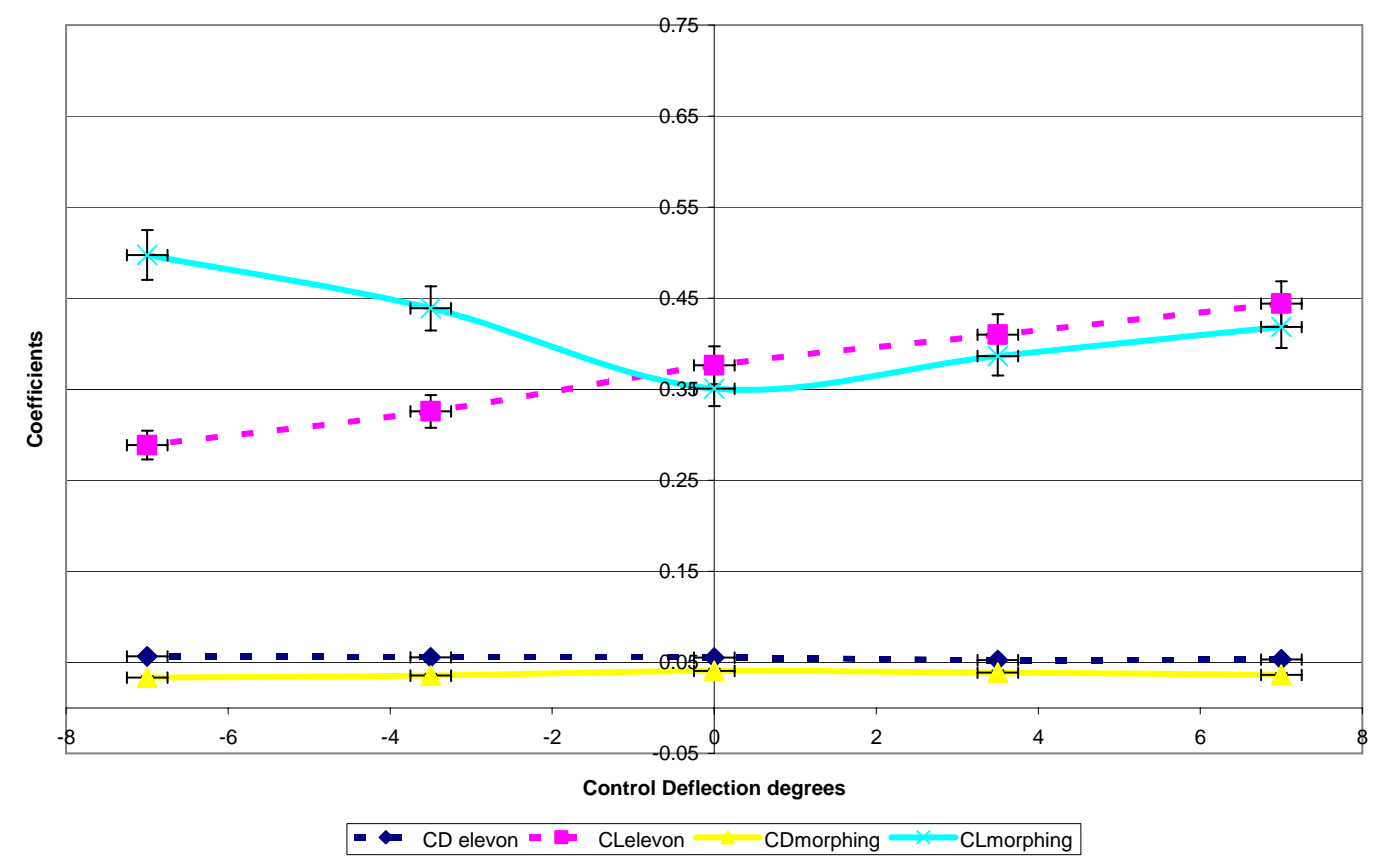

Figure 81- Experimental $C_{L}$ and $C_{D}$ for both wings at 7 degrees $A O A$ and $68 \mathrm{ft} / \sec (21 \mathrm{~m} / \mathrm{s})$.

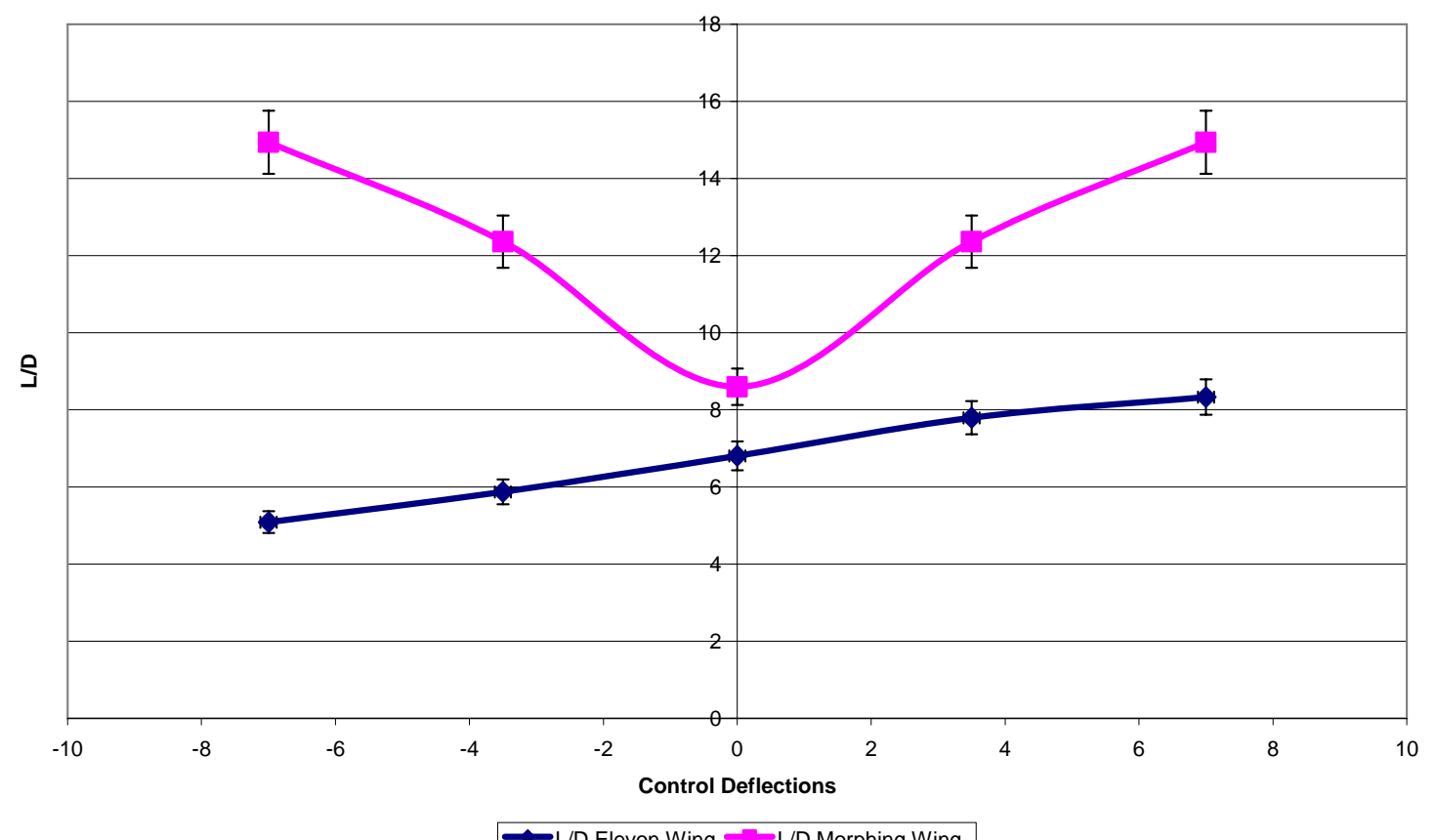

Figure 82- Experimental L/D ratios for both wings at 7 degrees $\mathrm{AOA}$ and $68 \mathrm{ft} / \mathrm{sec}(21 \mathrm{~m} / \mathrm{s})$. 
The behavior of yaw moments is very important to the control of any aircraft and is of particular interest when dealing with a tailless aircraft. Figure 83 shows the yaw behavior of both wings at 0 and 7 degrees AOA and $68 \mathrm{ft} / \mathrm{sec}(21 \mathrm{~m} / \mathrm{s})$. At zero AOA the behavior of both wings yaw appears to be opposite with much larger yaw changes and a reversal of yaw moment in the morphing wing. The yaw behavior of both wings becomes much more similar at 3.5 and 7 degrees AOA with the interesting point that at control positions 4 and 5 the yaw moment become significantly negative. Control positions 4 and 5 are the positions with the highest lift. This same unusual behavior can be seen in the $C_{D}$ of both wings as lift increases. This behavior is not seen at 10 and $15 \mathrm{~m} / \mathrm{s}$. This may indicate that there is a Reynolds (Re) number dependence. A full set of data for the elevon wing was collected at $26 \mathrm{~m} / \mathrm{s}$ and $\mathrm{Re}=493497$ to show that the trends seen at $21 \mathrm{~m} / \mathrm{s}$ remain at 26 $\mathrm{m} / \mathrm{s}$. (Table 6) The morphing wing wind tunnel model can not exceed $21 \mathrm{~m} / \mathrm{s}$ currently due to ballooning of the neoprene skin. To explore this possibility of a Re dependence the elevon wing was tested at control position 5 and 7 degrees AOA between Reynolds numbers roughly between 56,000 to 493,000, see Table 7 and Figure 140.

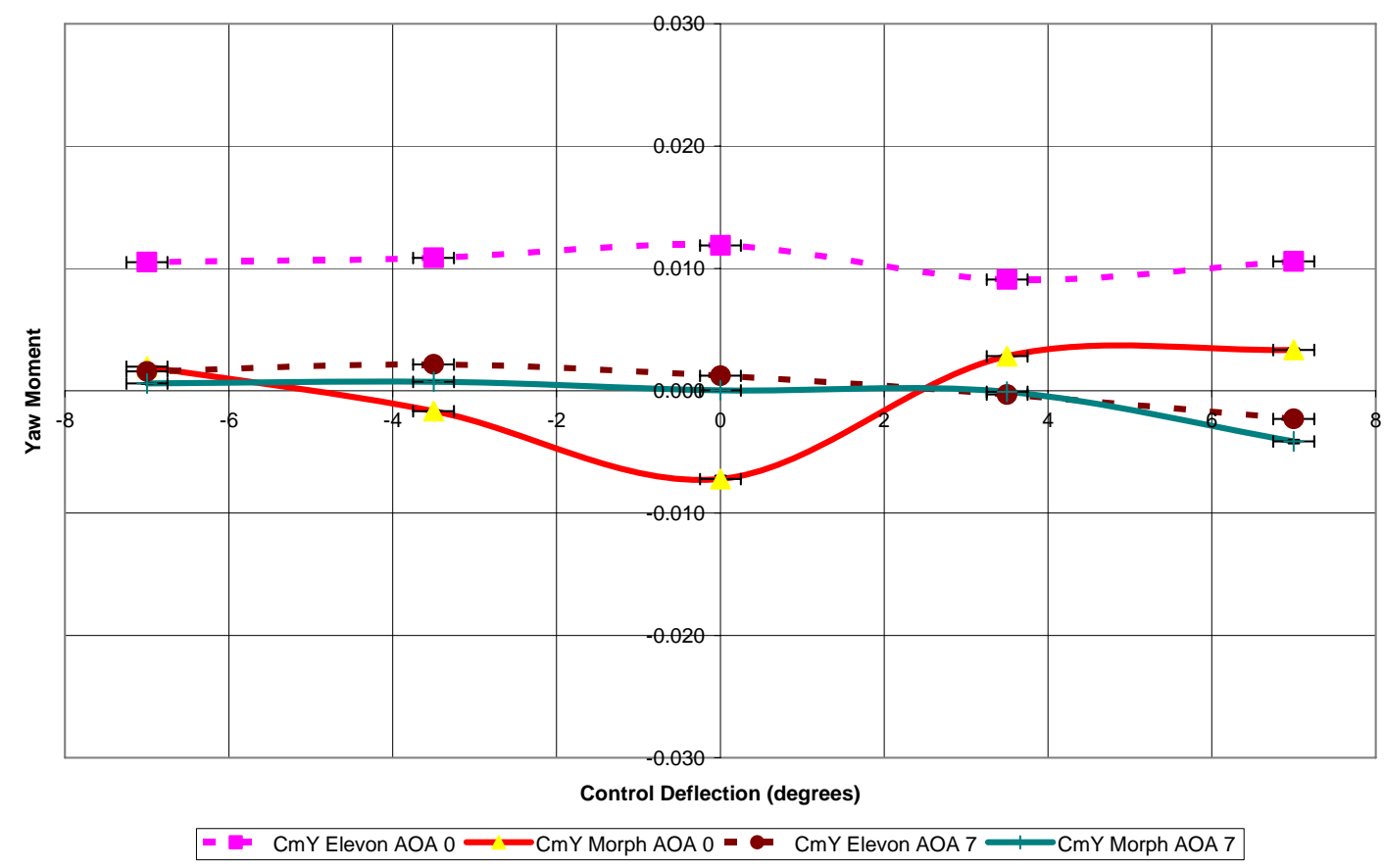

Figure 83- Experimental Yaw moment coefficients for both wings at 0 and 7 degrees $\mathrm{AOA}$ and 68 $\mathrm{ft} / \mathrm{sec}(20 \mathrm{~m} / \mathrm{s})$.

Control

Position

Table 6- Aerodynamic coefficients for the elevon wing at $26 \mathrm{~m} / \mathrm{s}$

1

2

3

4

5

\begin{tabular}{|c|c|c|c|c|}
\hline \multicolumn{1}{c}{$\mathbf{C}_{\mathbf{D}}$} & $\mathbf{C}_{\mathrm{mY}}$ & $\mathbf{C}_{\mathbf{m P}}$ & $\mathbf{C}_{\mathrm{mR}}$ & $\mathbf{C}_{\mathbf{L}}$ \\
\hline 0.039 & -0.002 & -0.102 & 0.139 & 0.432 \\
\hline 0.043 & -0.001 & -0.064 & 0.112 & 0.377 \\
\hline 0.046 & -0.001 & -0.036 & 0.093 & 0.346 \\
\hline 0.035 & -0.004 & -0.139 & 0.164 & 0.465 \\
\hline 0.035 & -0.007 & -0.171 & 0.179 & 0.501 \\
\hline
\end{tabular}

L/D 
Table 7- Aerodynamic coefficients for the elevon wing at control position 5 and varying velocities.

\begin{tabular}{|c|c|c|c|c|c|c|c|c|}
\hline $\begin{array}{l}\text { Tunnel } \\
Q \text { (psf) } \\
\end{array}$ & $\begin{array}{c}\text { Velocity } \\
(\mathrm{m} / \mathrm{s})\end{array}$ & Reynolds \# & $C_{D}$ & $C_{m Y}$ & $\mathrm{C}_{\mathrm{mP}}$ & $\mathrm{C}_{\mathrm{mR}}$ & $\mathrm{C}_{\mathrm{L}}$ & L/D \\
\hline 0.11 & 3.03 & 56417 & 0.166 & -0.031 & -0.229 & 0.240 & 0.771 & 4.65 \\
\hline 0.21 & 4.18 & 77865 & 0.119 & -0.020 & -0.229 & 0.231 & 0.709 & 5.97 \\
\hline 0.31 & 5.12 & 95368 & 0.120 & -0.019 & -0.223 & 0.225 & 0.673 & 5.62 \\
\hline 0.47 & 6.27 & 116805 & 0.122 & -0.015 & -0.237 & 0.243 & 0.712 & 5.86 \\
\hline 0.62 & 7.24 & 134880 & 0.129 & -0.014 & -0.244 & 0.250 & 0.730 & 5.64 \\
\hline 0.78 & 8.09 & 150807 & 0.115 & -0.012 & -0.223 & 0.227 & 0.653 & 5.66 \\
\hline 1.20 & 10.02 & 186760 & 0.102 & -0.010 & -0.209 & 0.214 & 0.609 & 5.96 \\
\hline 2.70 & 15.06 & 280918 & 0.077 & -0.009 & -0.214 & 0.219 & 0.615 & 8.03 \\
\hline 4.84 & 20.12 & 375877 & 0.054 & -0.008 & -0.206 & 0.212 & 0.601 & 11.06 \\
\hline 7.44 & 24.94 & 466387 & 0.038 & -0.007 & -0.182 & 0.195 & 0.524 & 13.86 \\
\hline 8.32 & 26.37 & 493437 & 0.035 & -0.007 & -0.171 & 0.179 & 0.501 & 14.38 \\
\hline
\end{tabular}

\section{Flow Visualization}

Various flow visualization techniques were experimented with in order to better understand the flow on the outer third of both wing models. The most promising technique was the use of glycerin smoke. Various wands and rakes have been fabricated in order to introduce the smoke into the flow field. Using a 2 watt $\mathrm{C} \mathrm{W}$ Argon laser to produce a laser sheet by passing the laser through a $1 / 4$ inch diameter acrylic rod has been partially successful when using a transparent model. (Figure 84), but it appears that the smoke has difficulty being entrained into the boundary layer. Similar visualization on another project where the smoke is plumbed through the wing and then is released directly into the boundary layer has had spectacular results.

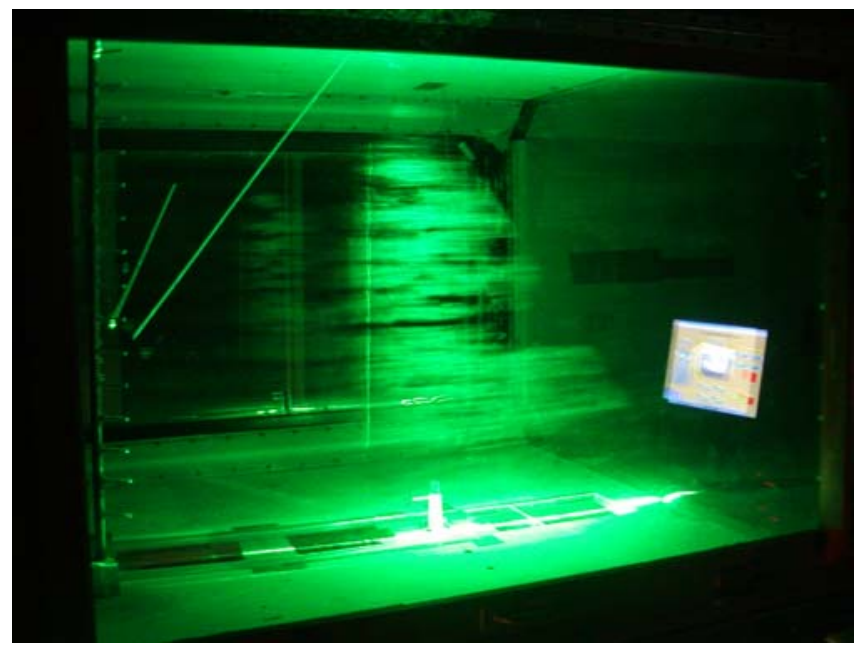

Figure 84- Experiments with a 2 watt laser sheet and glycerin smoke. Notice the smoke rake and transparent cylinder model. 
Smoke flow visualization has the potential of significantly aiding the understanding of the flow in these tailless models. While many new techniques were developed very little was learned about the flow structure. The models were also tufted using polyester tufts on a 1.5 inch grid. Tufts have the advantage that the flows at the test speed where data is being acquired can be visualized. Both the elevon and morphing models were filmed at $68 \mathrm{ft} / \mathrm{sec}(21 \mathrm{~m} / \mathrm{s})$ through all five control positions and with the wing at 7 degrees angle of attack.

Observations under these conditions show that the majority of the flow is laminar. The onset of separated flow starts to become visible at the root of the elevon at control position 5 on the PUL-10 model. The tip vortices can clearly be seen with tufts. The tip vortex on the elevon equipped wing disappears between control position 1 and 2 . When facing the leading edge left wing wind tunnel model the vortices are counter clockwise at control position 3 and clockwise at control positions 3, 4 and 5. The tip vortex on the morphing wing disappears at control position 2 . When facing the leading edge left wing wind tunnel model the vortices are counter clockwise at control position 3 and clockwise at control positions 1, 4 and 5, as seen on Figure 85, Figure 86, Figure 87,Figure 88 and Figure 89. Although it is difficult to quantify there appears to be unusual behaviour in the up wash at the tip of the morphing wing. The AOA of the wing tip seems to remain at in a small range with the local flow even when the tip is +14 degrees AOA to the tunnel flow.

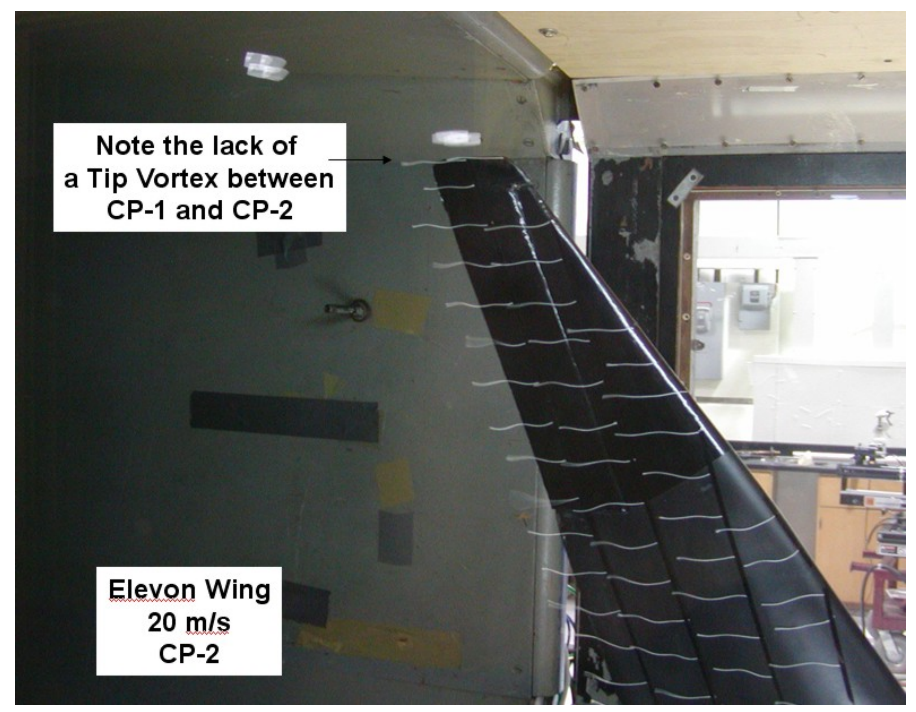

Figure 85- Elevon Wing at $68 \mathrm{ft} / \mathrm{sec}(21 \mathrm{~m} / \mathrm{s}), 7$ degree AOA and with a +3.5 degree control deflection 2. 


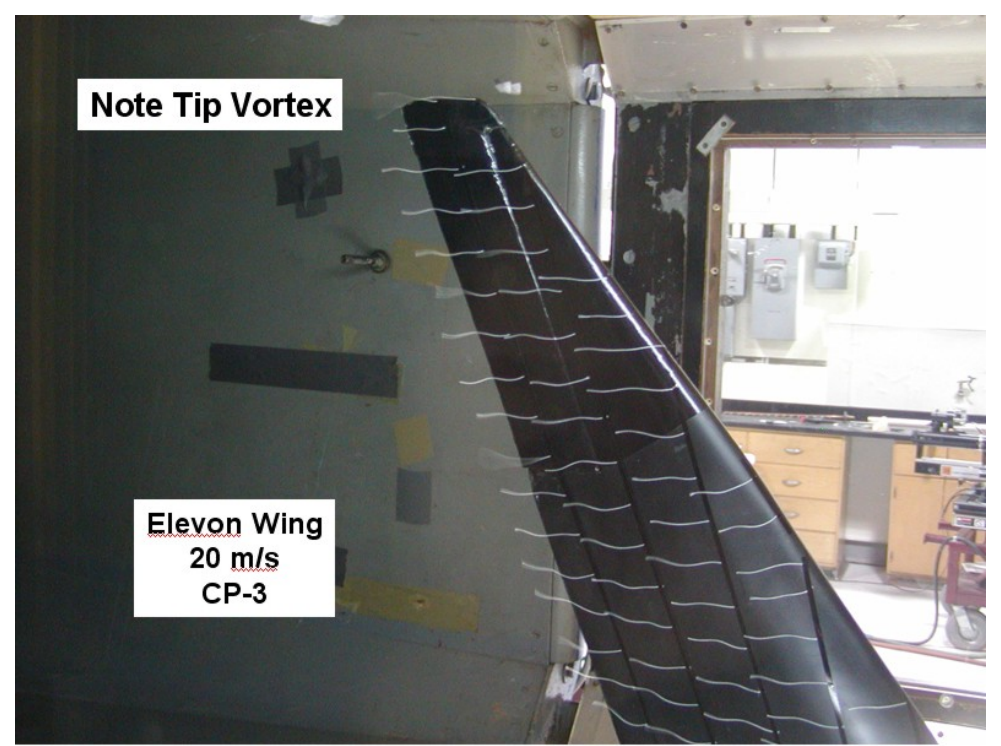

Figure 86- Elevon Wing at $68 \mathrm{ft} / \mathrm{sec}(21 \mathrm{~m} / \mathrm{s}), 7$ degree AOA and with a +7 degree control deflection.

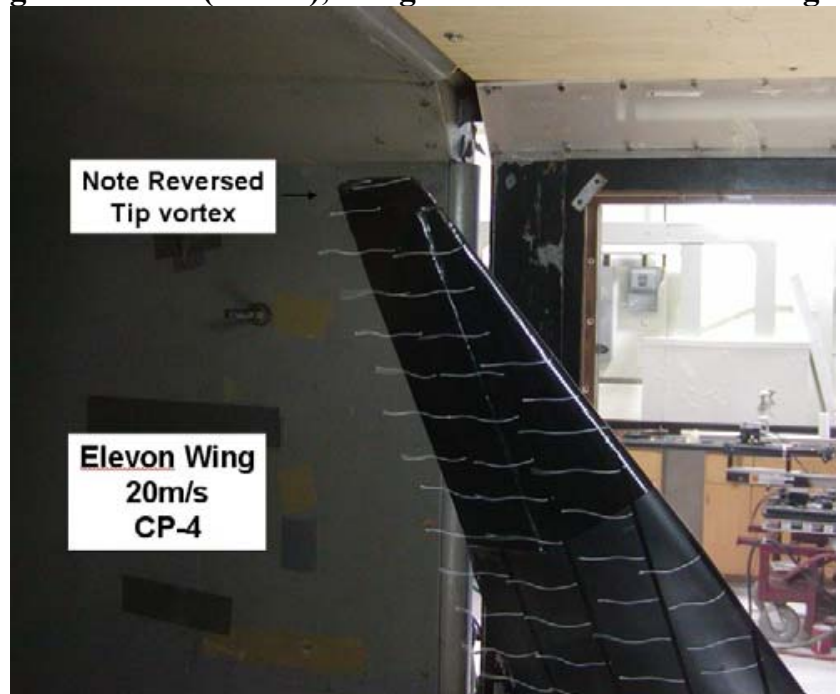

Figure 87- Elevon Wing at $68 \mathrm{ft} / \mathrm{sec}(21 \mathrm{~m} / \mathrm{s}), 7$ degree AOA and with a -3.5 degree control deflection.

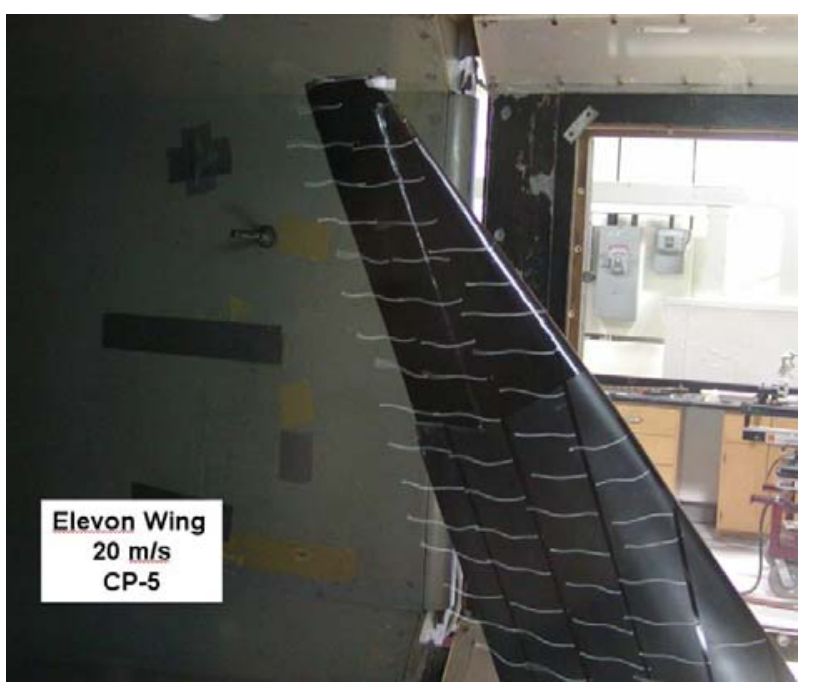


Figure 88- Elevon Wing at $68 \mathrm{ft} / \mathrm{sec}(21 \mathrm{~m} / \mathrm{s}), 7$ degree AOA and with a +7 degree control deflection. Note disturbed flow at the root of the elevon.

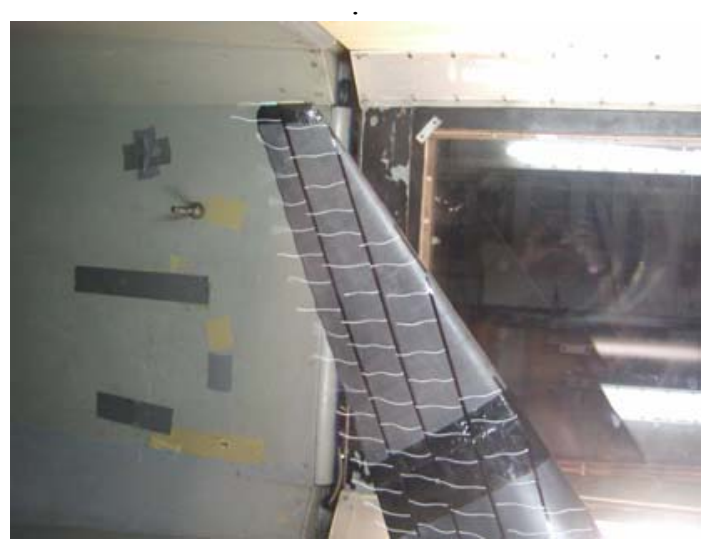

Figure 89- Morphing Wing at $68 \mathrm{ft} / \mathrm{sec}(21 \mathrm{~m} / \mathrm{s}), 7$ degree AOA and with an approximate -2 degree control deflection. Note lack of tip vortex

Because of the inability of smoke to enter the boundary layer of the flow over the wind tunnel models and to due to the relatively high velocities needed it was decided to introduce a higher viscosity fluid to the boundary layer. Tempura paints with metallic flecks was painted on the wind tunnel model and tested with mixed results. Some flow structures could be seen by eye but were very difficult to photograph. After over 30 tests with different fluids, it was found that fluorescent Tempura paint could be photographed with the use of a ultraviolet light. There were two problems with this paint; one was that it had too high a viscosity and its surface tension was too great, so it beaded up on the wing surface. The viscosity was modified by diluting with water and the surface tension was modified by using a vegetable oil additive. The new fluorescent flow visualization fluid was easily entrained in the flow and could be photographed using ultraviolet lights. Another problem encountered was that the wind tunnel models were oriented in the wind tunnel with their span vertical. This orientation introduced a gravity component to the fluid flow once the fluid began to move to due to the air velocity. A new horizontal variable AOA wind tunnel mount was designed and constructed. The NACA 0015 validation model, the morphing wing and the non-morphing wing could all be mounted from -10 to +12 AOA at the same time as the entire range of control surface movements can be varied. Currently the viscosity of the flow visualization fluid is being experimented with. This flow visualization technique was hoped to provide the ability for the measurement of flow vector direction, location of the stagnation point on the leading edge (indication of upwash) and indication of separated flow regions, but was not as successful as expected. This technique turned out to be purely qualitative and results lacked repeatability. A general observation, which may be due to the fluid properties of the fluorescent tempura paint, was that when the morphing wing was at high angle $\mathrm{s}$ of attack with a control deflection between +3.5 and +7 degrees a scallop pattern developed along the outboard one third of the wing. (Figure 90 through Figure 92) 


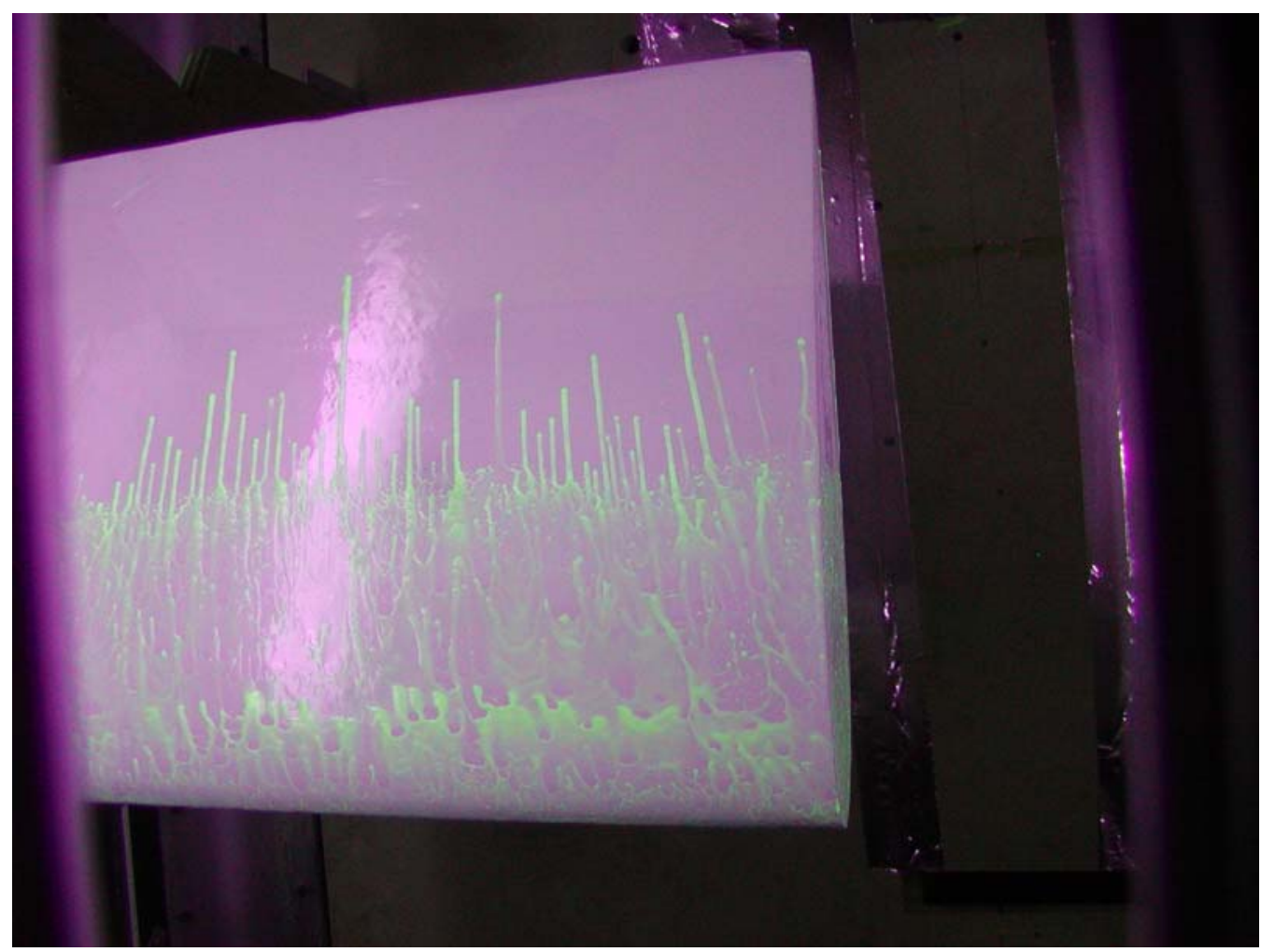

Figure 90 - Florescent oil flow visualization at $21 \mathrm{~m} / \mathrm{s}$ with rectangular wing using theNACA 0015 airfoil.

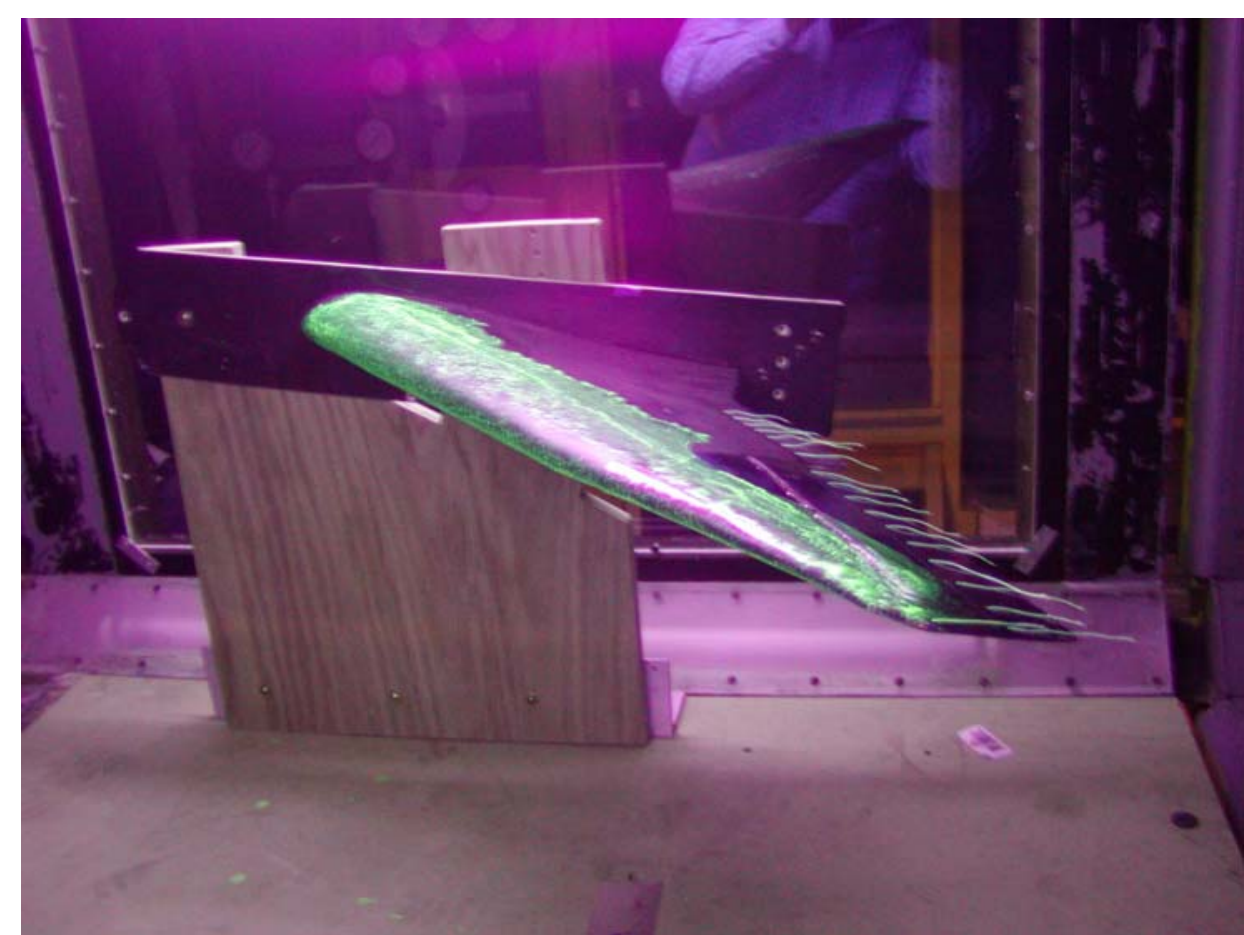

Figure 91- Non-morphing wing with florescent flow visualization fluid at 7 degrees AOA, control position 5 and $21 \mathrm{~m} / \mathrm{s}$. 


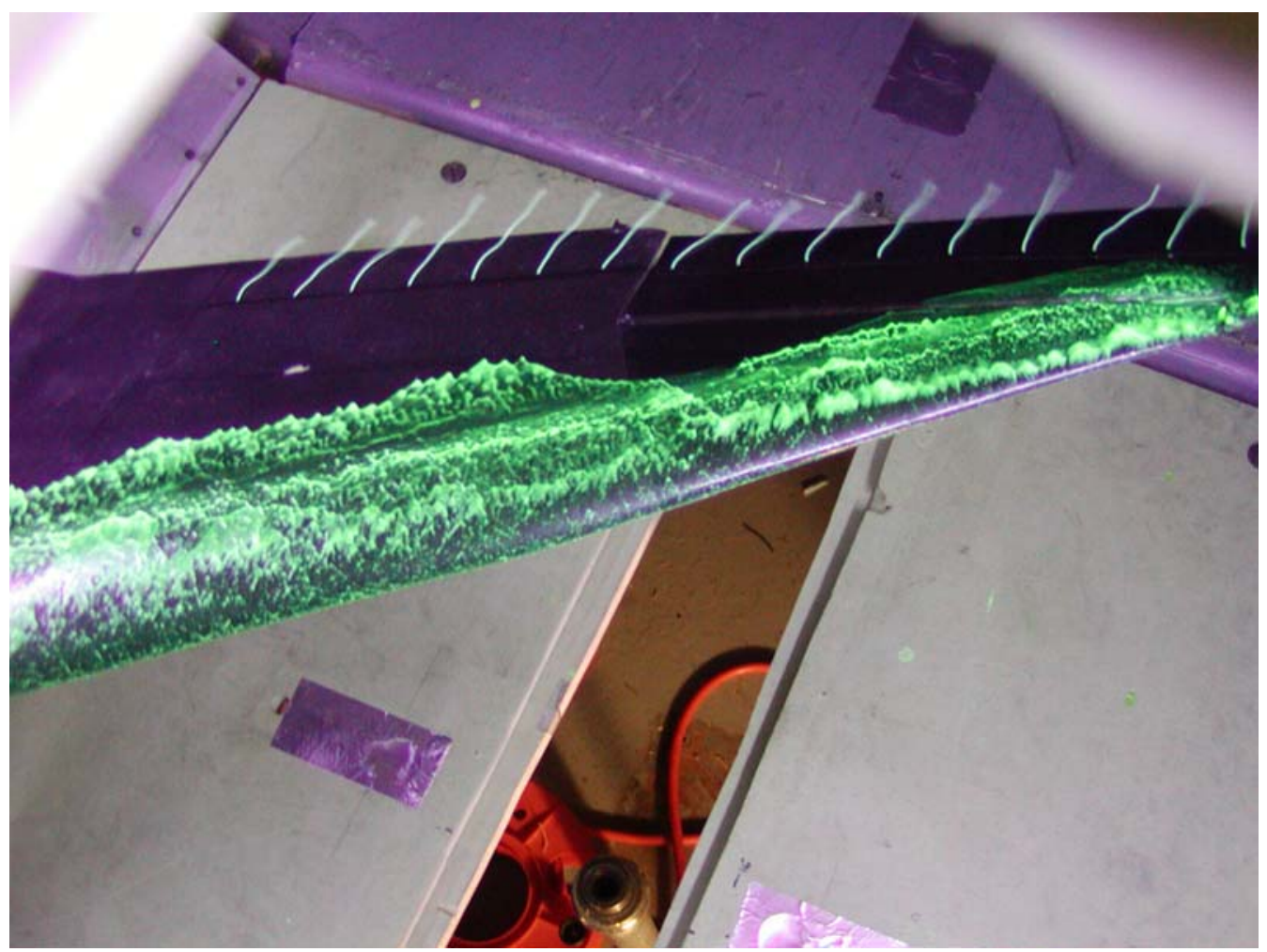

Figure 92-Wing with florescent flow visualization fluid at 7 degrees AOA, control position 5 and 21 $\mathbf{m} / \mathbf{s}$.

\section{Computational Fluid Dynamic studies}

In order to gain greater insight into the flow physics of the morphing wing, CFD models of the various control positions at zero degrees angle of attack and seven degrees angle of attack were developed. In parallel with the development these models a validation model, to prove out the techniques used, was developed. There have been a number of computational studies on 3D wings. Data from two of these studies were available to this project for model validation. One of these studies is used as the standard swept wing CFD validation study for NASA. The NASA study utilizes an ONERA M6 wing at a Reynolds number of 11 million. (Figure 94) The other study, "Prediction of Tip-Vortex Flow Past a Finite Wing" by Sung-Eun Kim and Shin Hyung Rhee from the Fluent Corporation uses a straight rectangular wing with a NACA 0012 airfoil profile at a Reynolds Number of 4.6 million. Both studies have Reynolds numbers far too high for the morphing wing project's value of 375,000 but the Kim and Rhee has been chosen as the validation study for the CFD work conducted in this research because of its simple geometry and lower Re. Kim and Rhee's study was conducted in 2005 and attempted to match the flow physics and data collected by Jim Chow of NASA Ames Research Center. Chow conducted extensive wind tunnel studies on a low aspect ratio rectangular wing model at a Reynolds number of 4.6 million. This study was conducted in a NASA low speed wind tunnel with a 32 " $x 48$ " test section. The model was half wing with a rounded tip, a semispan of 36 " and a chord of 48 ". Detailed surface pressures as well as wake vorticity and pressure measurements were taken at an angle of attack of 10 degrees. (Figure 93) 
Velocity field measurements were obtained with a seven hole pressure probe, two-point single wire, and turbulence measurements with a three-wire probe. The model had 222 static pressure taps to record the surface pressure. Integration of the pressure data yielded a coefficient of lift of 0.51 and a large axial velocity of $1.77 * \mathrm{~V}_{\infty}$ in the vortex core at the wing tip. The density of data collected in this study made it ideal to try to match with a computational model.

Kim and Rhee created a model which matched Chows experimental geometry. Then they used this model in the commercial Computational Fluid Dynamics code "Fluent" to evaluate a variety of meshing techniques as well as turbulence closure techniques. Models were developed which showed good agreement with Chows data. The low aspect ratio wing was meshed using a quad (rectangular) grid with of varying total for the tunnel and wing, cell counts from approximately a 385,000 cells to 2.3 million cells both globally and locally defined. Boundary conditions were defined as a velocity inlet, a pressure outlet, generalized wall function for the wing surface and moving walls for the tunnel walls. The coarsest mesh of 385,000 showed all of the major qualitative features of the flow, but under predicted static pressures and the maximum axial velocity of the tip vortex. The 2.3 million cell model gave good predictions of every aspect of the flow. A locally adapted 1.4 million cell model despite much fewer cells showed a level of accuracy very close to the finest mesh. The two best performing turbulence models were the differential Reynolds Stress Tensor "Seven equation" Model (RSTM) and the SpalartAllmaras Model (S-A) Both second order and QUICK differentiating techniques were used.
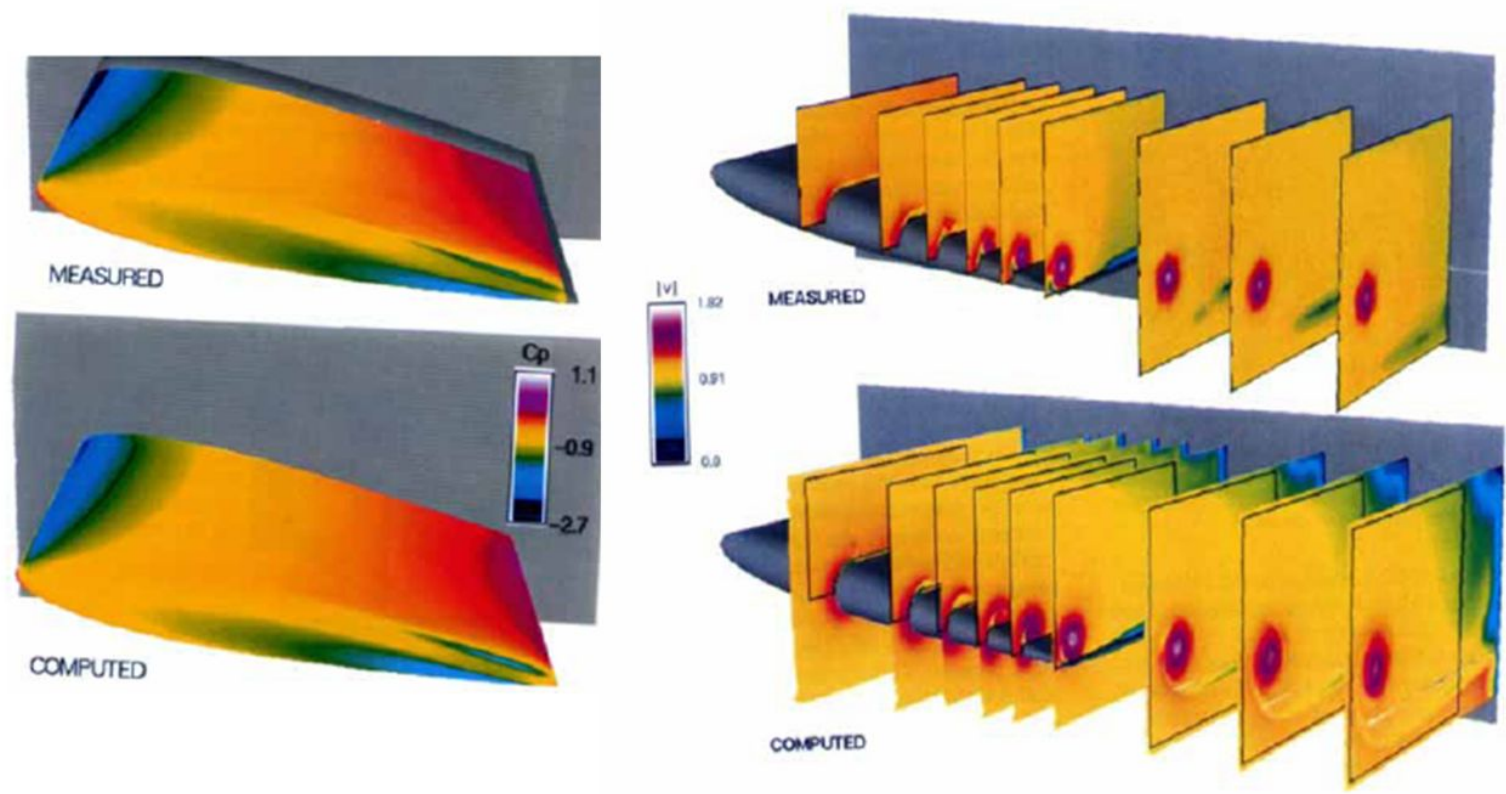

Figure 93-Pressure field measured by Chow and modeled by Dacles and Mariani.

A similar model to Kim and Rhee's was developed to be used as a validation model for the morphing wing models for this project. Creation of the CFD validation model was 
conducted in parallel with the creation of the morphing wing models in order to ensure the functionality of the meshing and modeling techniques used for the morphing models. All model geometries were initially created in the Solid Works CAD package. Then these geometries were imported into Fluent's geometry and meshing development code "Gambit". Interoperability issues between Solid Works and Gambit required all the geometries to be redeveloped in Gambit. A variety of meshing techniques were experimented with on both the low aspect ratio rectangular NACA0012 wing as well as the morphing wing models from this project. The quad grid used in Kim and Rhee's study worked well on the rectangular wing, but created problems when it was adapted to the swept wing configuration of the morphing models. Gambit could not generate an efficient high quality mesh which allowed a fine quad grid on the wing to be transitioned to a quad or tri grid of the wind tunnel walls without generating an extremely fine inefficient mesh.

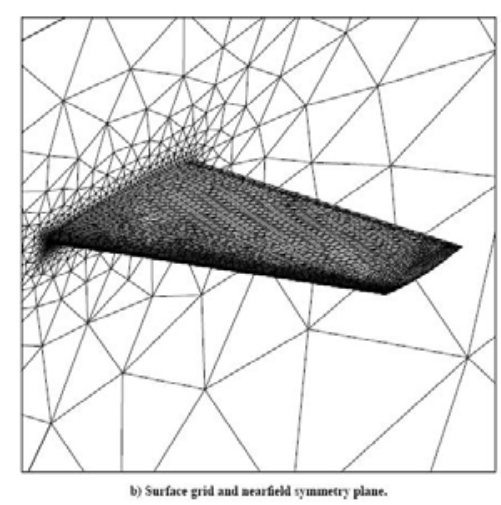

Figure 94- ONERA M6 Wing mesh used in NASA Ames CFD validation study.

At this point meshing techniques from a widely used swept wing CFD validation model developed by NASA became available. This model of a 35 degree swept ONERA-M6 wing has been used numerous times in literature for CFD validation. The NASA model was developed by John Slater from NASA Glenn Research Center in 2002. Although this model was designed to study transonic flow the meshing technique used was excellent for the swept morphing wing models. A relatively fine triangular mesh on the wing which transitions to a Tet (Mostly triangular) Hybrid Tgrid in the tunnel section. (Figure 95 and Figure 96) This technique was then applied to both the validation model and the morphing wing. Mesh quality was examined using Gambits built in analysis tools. It was found that certain ratios of fine wing face grid to the coarser volume grid used in the tunnel performed better than others. Certain ratios could not even be generated with out errors. Ratios of wing grid intervals to volume grid intervals of $1: 2,1: 3$, or $1: 5$ all appeared to work well. 


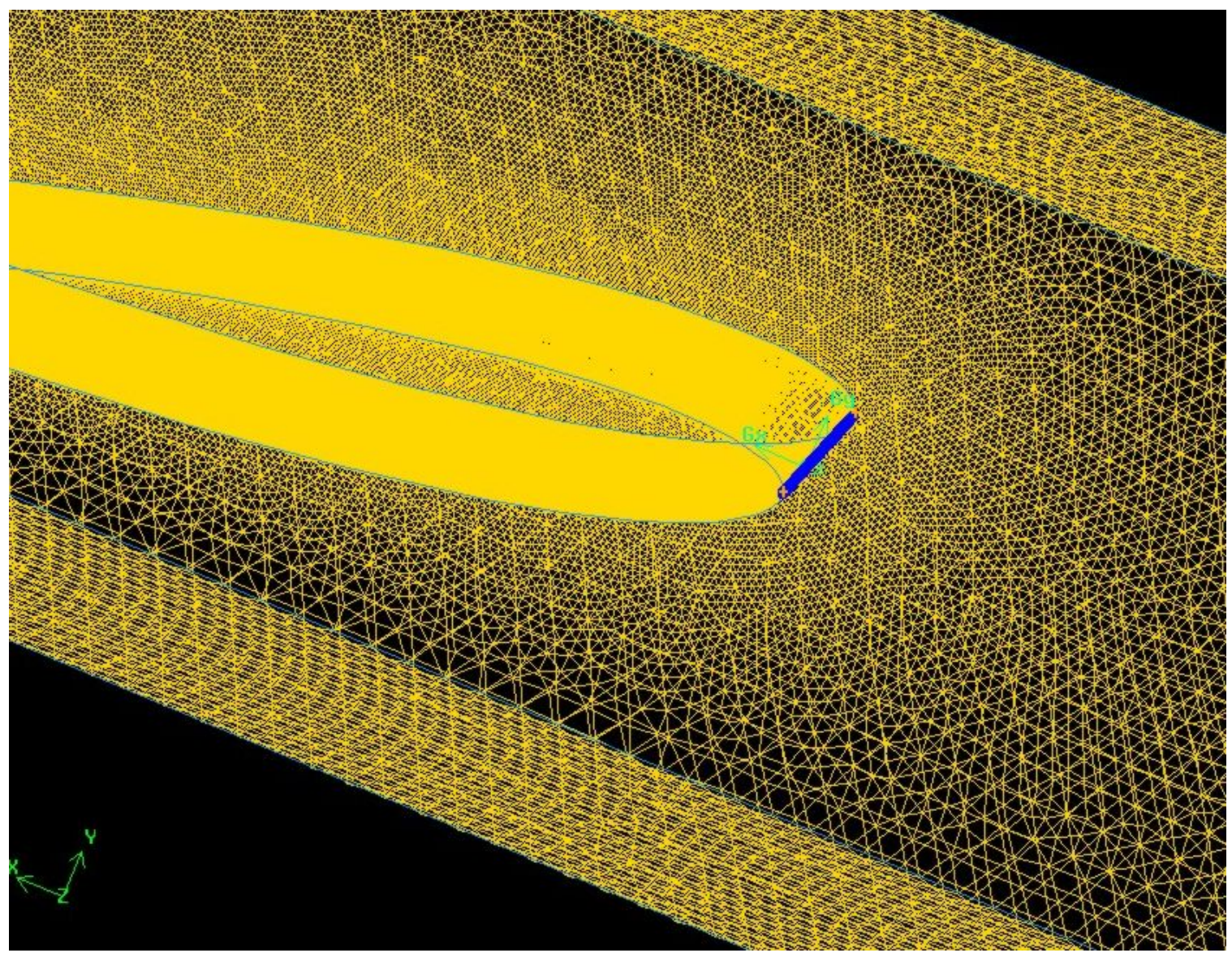

Figure 95- Validation Grid used on the Kim and Rhee type model.

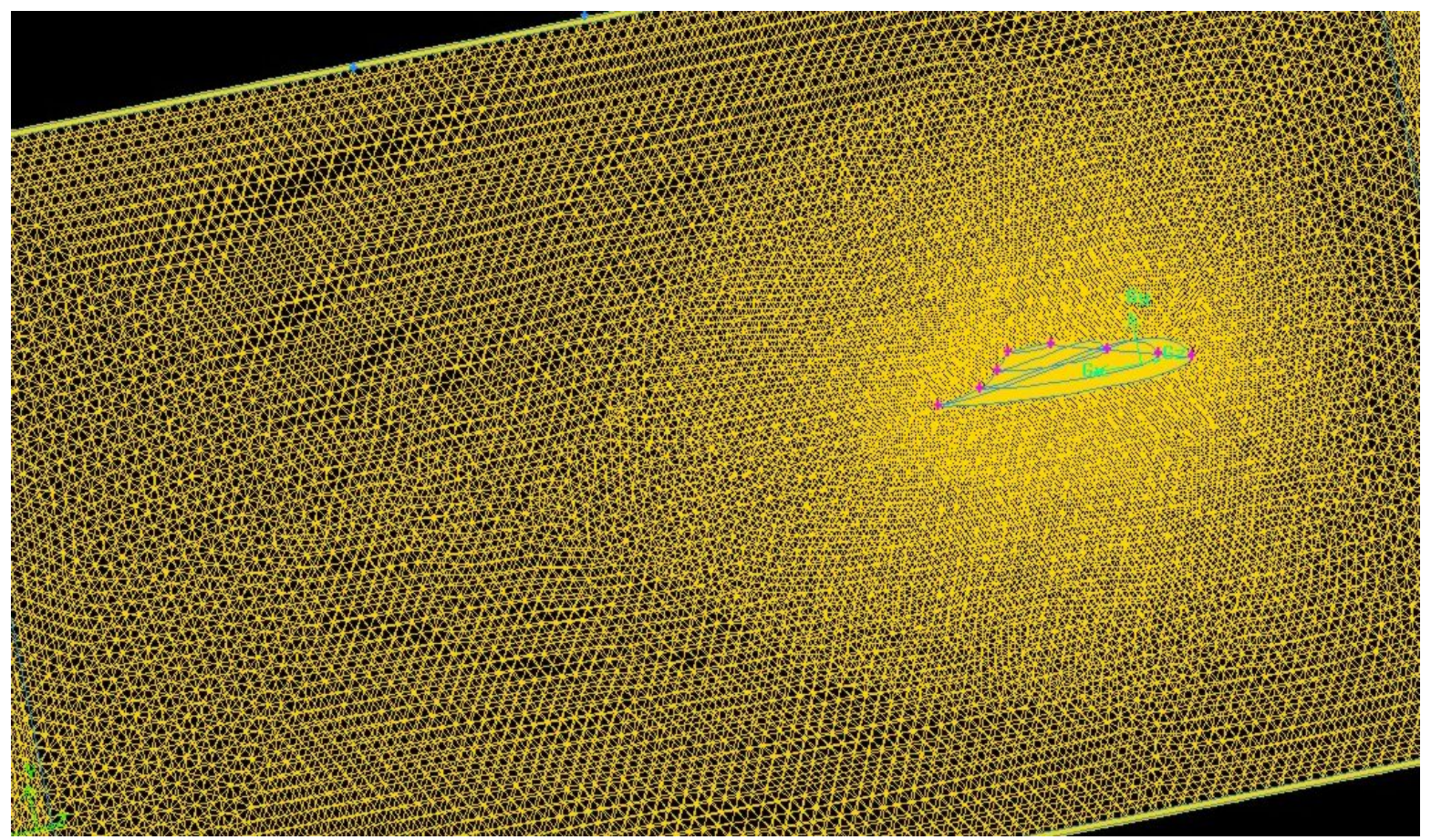

Figure 96- Initial morphing wing mesh 1.6million cells 
At this point work on the validation model continued forward to determine the best boundary conditions and cell intervals for the model. Boundary conditions were defined as a velocity inlet, a pressure outlet, generalized wall function for the wing surfaces and the tunnel walls. Justification could not be found for the use of the moving wall boundary condition for the tunnel walls that Kim and Rhee used because this study is comparing sets of wind tunnel data, this type of model was also experimented with as well as laminar flow. Models with cell counts from 1.28 million to 4.78 million were generated and imported into Fluent 6 as mesh files. The models generated had the same basic geometry as Kim and Rhee's with a 32" x 48" x $19 \mathrm{ft}$ test section and a 36" x 48" rectangular wing using the NACA0012 airfoil with a round tip.

Due to computing power limitations and the good performance shown by Kim and a Rhee the Spalart-Allmarus Model (S-A) turbulence model was used. Because these models are approximating wind tunnel conditions the "Turbulence Intensity and Hydraulic Diameter" factor was used as the turbulence specification method for both the velocity inlet and the pressure outlet. The discretization of the momentum and modified turbulent viscosity was also done by second order upwind schemes at the recommendation of Fluent. This initial setup was followed by experimentation with a laminar model and with the use of Fluent's pressure based dynamic adaptive grid. All models were run with the velocity inlet velocity set at $170 \mathrm{ft} / \mathrm{s}$ and were limited to approximately 2.2 million cell due to available computing power.

Table 8 - $C_{L}$ and $C_{D}$ of various Fluent models simulating Chows experiments

\begin{tabular}{|c|c|c|c|c|}
\hline Model & Mesh and Boundaries & $\mathrm{C}_{\mathrm{L}}$ & $C_{D}$ & $\begin{array}{c}\text { Wing Pressure } \\
\text { Contours }\end{array}$ \\
\hline Chow (Experimental Data) & NA & 0.51 & 0.077 & $\begin{array}{l}\text { Baseline - } 220 \\
\text { Pressure Taps }\end{array}$ \\
\hline NACA0012REVA626 & $\begin{array}{l}1.6 \text { million cells Spalart-Allmaras, } \\
\text { 2nd Order }\end{array}$ & 0.78 & 0.1 & \\
\hline $\begin{array}{l}\text { NACA0012REVA629 used adapive } \\
\text { boundary grid on wing and dynamic } \\
\text { pressure gradient adaptive grid }\end{array}$ & $\begin{array}{l}1.6 \text { million cells Spalart-Allmaras, } \\
\text { 2nd Order }\end{array}$ & 0.76 & 0.131 & \\
\hline $\begin{array}{l}\text { NACA } 0012 \text { Extended } 701 \text { Moved walls out } \\
\text { by } 4 \text { cord lengths }\end{array}$ & $\begin{array}{c}1.9 \text { million cells Spalart-Allmaras, } \\
\text { 2nd Order }\end{array}$ & 0.425 & 0.079 & Excellent match \\
\hline $\begin{array}{c}\text { NACA0012709 used adapive boundary grid } \\
\text { on wing and dynamic pressure gradient } \\
\text { adaptive grid }\end{array}$ & $\begin{array}{l}0.8 \text { million cells Spalart-Allmaras, } \\
\text { 2nd Order }\end{array}$ & 0.76 & 0.106 & \\
\hline $\begin{array}{l}\text { NACA0012REVA629709 used adaptive } \\
\text { boundary grid on wing and dynamic } \\
\text { pressure gradient adaptive grid }\end{array}$ & $\begin{array}{l}2.17 \text { million cells Spalart- } \\
\text { Allmaras, } 2 \text { nd Order }\end{array}$ & 0.76 & 0.131 & \\
\hline $\begin{array}{l}\text { NACA0012Extended } 710 \text { used adaptive } \\
\text { boundary grid on wing and dynamic } \\
\text { pressure gradient adaptive grid }\end{array}$ & $\begin{array}{l}2.17 \text { million cells Spalart- } \\
\text { Allmaras, 2nd Order }\end{array}$ & 0.43 & 0.081 & Excellent match \\
\hline $\begin{array}{l}\text { NACA0012709AL used adaptive boundary } \\
\text { grid on wing and dynamic pressure gradient } \\
\text { adaptive grid }\end{array}$ & $\begin{array}{l}2.17 \text { million cells Laminar, 2nd } \\
\text { Order }\end{array}$ & 0.67 & 0.062 & Excellent match \\
\hline
\end{tabular}


In general, without using the using moving wall boundary condition on the tunnel walls the prediction of $C_{L}$ and $C_{D}$ was high except for the laminar case which can only be poorly justified at a Reynolds number of 4.6 million and an AOA of 10 degrees. Due to these high predictions it was hypothesized that the calculated wall effects were the cause for the high aerodynamic coefficients. To test this idea two models were created with the tunnel walls widened by 4 cord lengths. The results of these models were excellent along with a model which used the pressure adaptive grid having the best results in duplicating pressure field seen in Chows data and Kim and Rhee's models.

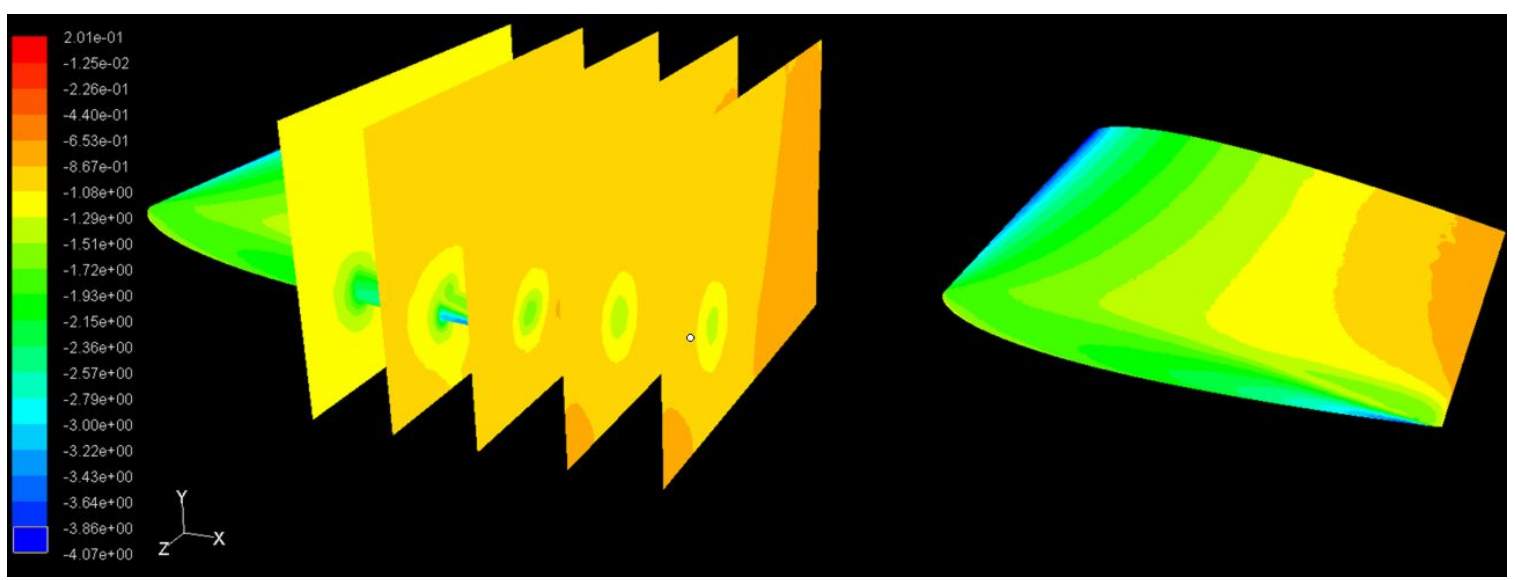

Figure 97-CFD validation model pressure field from the NACA 0012 Laminar model which utilized a static pressure gradient adaptive grid at $\mathbf{2 . 1 7}$ million cells.

In Chow's experiments the relative large size of his wing model when compared to the wind tunnel test section may be the cause of the difficulty in fitting a turbulence model because of blockage and wall effects. The wall effects combined with the model wind tunnel blockage may increase the local velocities and Reynolds numbers. The results of this experiment was to validate a both the grid cell intervals used and the dynamic pressure adaptive grid which will be used on the morphing wing models. 


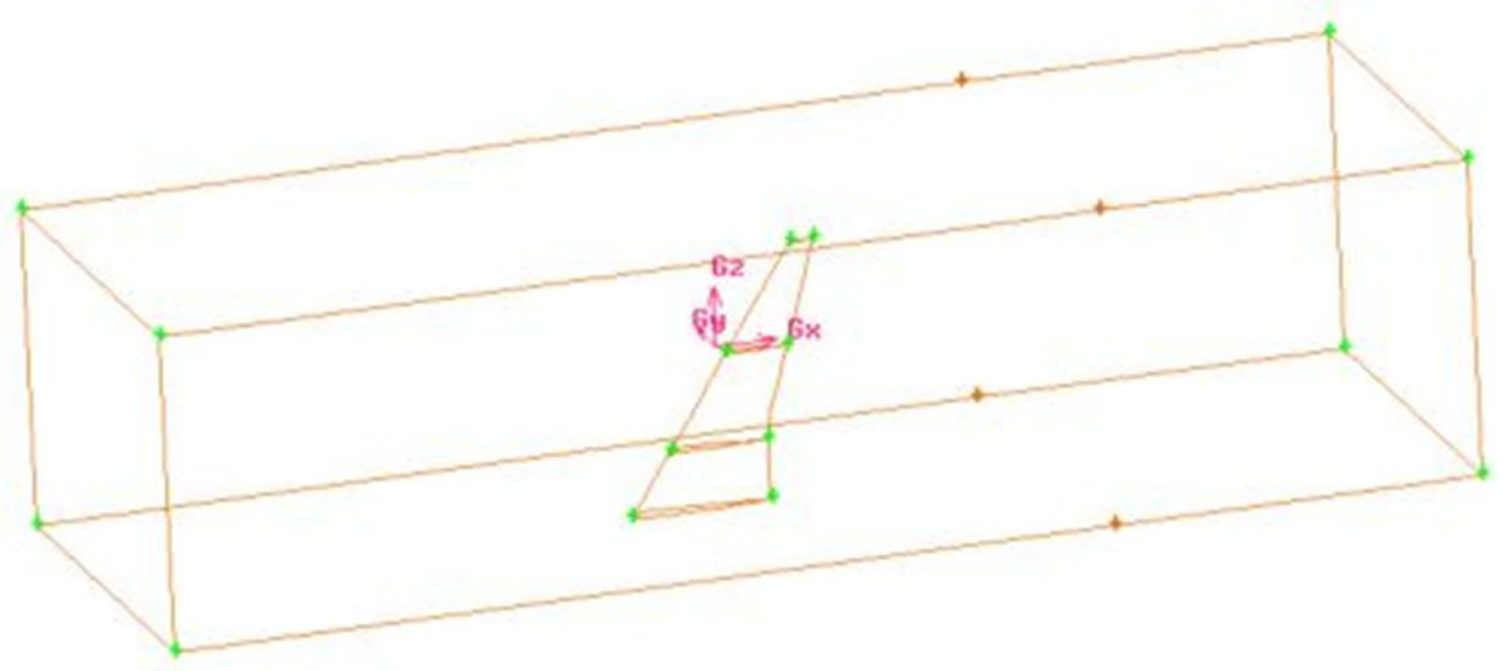

Figure 98 - Morphing wing model in CFD test section generated in GAMBIT.

The creation of CFD models for the morphing wing follows a similar path to that of the validation models. The geometry was constructed in GAMBIT. (Figure 98) The initial models were constructed with a cell interval spacing of $5 \mathrm{~mm}$ in the wing and $25 \mathrm{~mm}$ in the wind tunnel which gives an overall cell count in the 1.6 million range .Fluent's mesh analysis tools were used to evaluate the quality of each new mesh as shown in Figure 99. A velocity of $68 \mathrm{ft} / \mathrm{s}$ was set into the inlet boundary condition which matches one of the velocities used in the wind tunnel testing. The CFD model test section has the same 32" $\mathrm{x}$ 45 " cross section as the wind tunnel, but the 46.5 inch test section length was tripled in order to aid in meshing and to give time for Fluent to equilibrate the flow conditions before the outlet. Control positions at 0 degrees AOA and at 7 degrees AOA were built into 10 models (two angles of attack and 5 control positions) and were run using the Spalart-Allmaras turbulence model. Convergence occurred usually within 500 iterations. Typical convergence with these models can be seen in Figure 100.

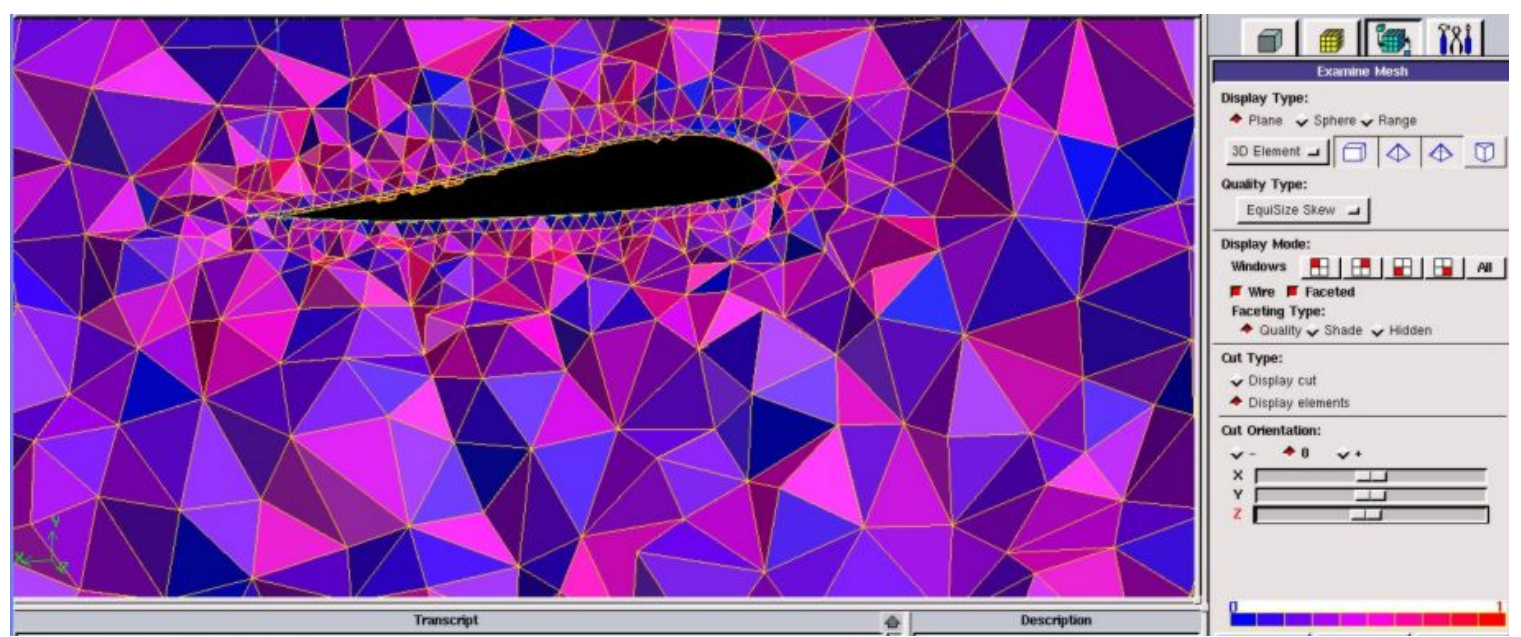

Figure 99 - Morphing wing model mesh quality analysis. 

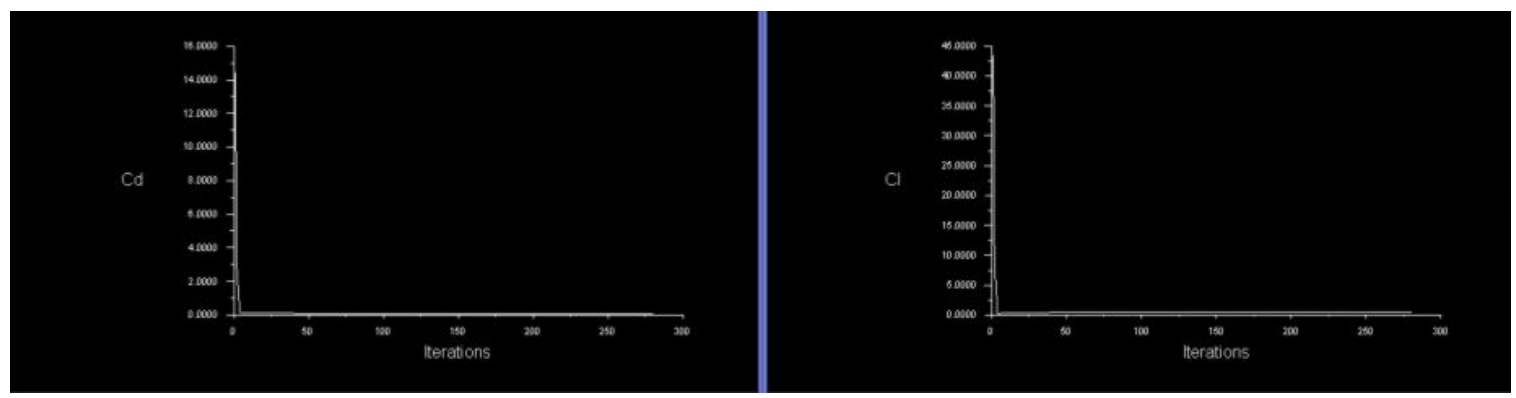

Figure 100 - Typical convergence monitors for an initial morphing wing model

In general the initial CFD models were over predicting both $\mathrm{C}_{\mathrm{L}}$ and $\mathrm{C}_{\mathrm{D}}$ for every condition when compared to wind tunnel data. Three changes were made at this point. The low Reynolds number of 375,000 used in these experiment s as well as the low angle of attack of the wing make a good argument for the use of the laminar model. At this point it was decided to utilize the adaptive grid function on the wing zones which added on average 160,000 grid cells to the wing model. This was done in conjunction with the. the dynamic pressure gradient adaptive grid function which was set to re-smooth the grid cell in zones with high pressure gradients every 20 iterations. The effect of the adaptive grid functions can be seen in Figure 101. The effect of the use of the dynamic pressure gradient adaptive grid can be seen on the convergence of $C_{L}$ in Figure 102.

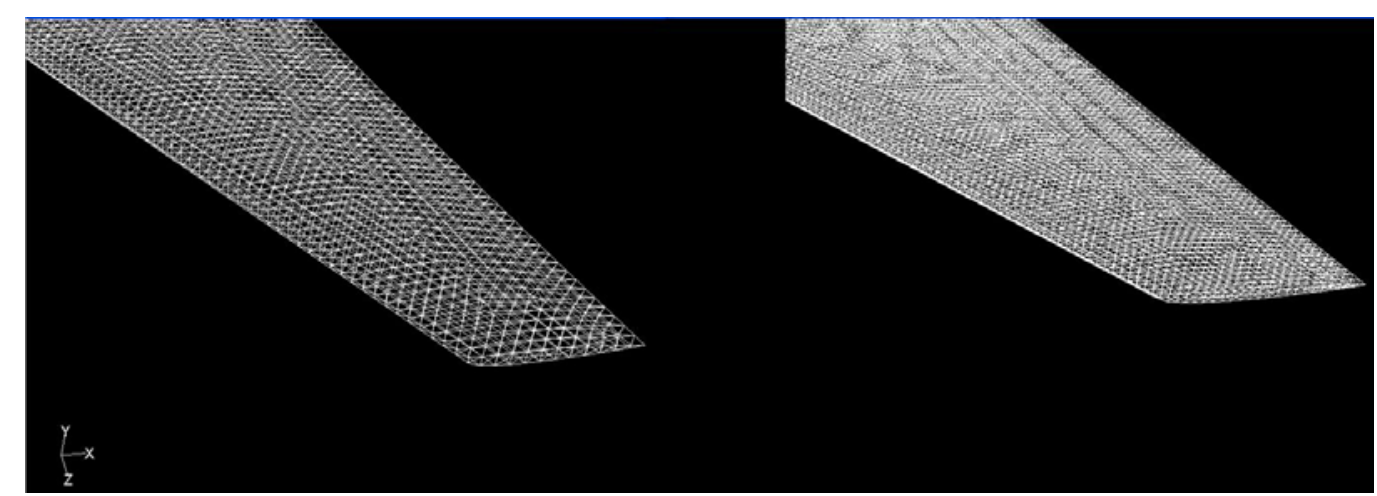

Figure 101 - Tip of the morphing wing model before and after the use of Fluent's adaptive grid functions.

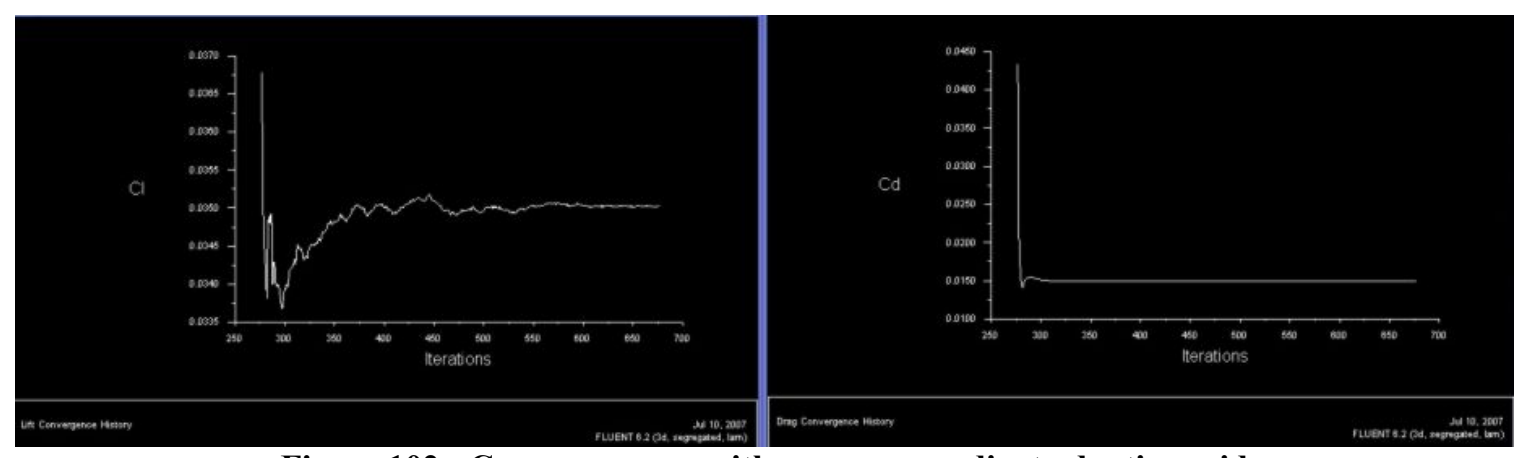

Figure $102-C_{L}$ convergence with pressure gradient adaptive grid 
Use of the laminar model along with the adaptive grid brought both $\mathrm{C}_{\mathrm{L}}$ and $\mathrm{C}_{\mathrm{D}}$ in line with the wind tunnel data. $C_{L}$ was still slightly high, but reasonable. $C_{L}$ and $C_{D}$ data for the various models is summarized in Table 1 and in Figure 103 through Figure 106.

Table 9 - Lift and Drag from Wind tunnel experiments $\left(C_{D} / C_{L}-W T\right)$, Turbulent $\left(C_{D} / C_{L}-S-A\right)$ and Laminar CFD $\left(\mathrm{C}_{\mathrm{D}} / \mathrm{C}_{\mathrm{L}}\right.$-LAM)models at 0 and 7 degrees AOA.

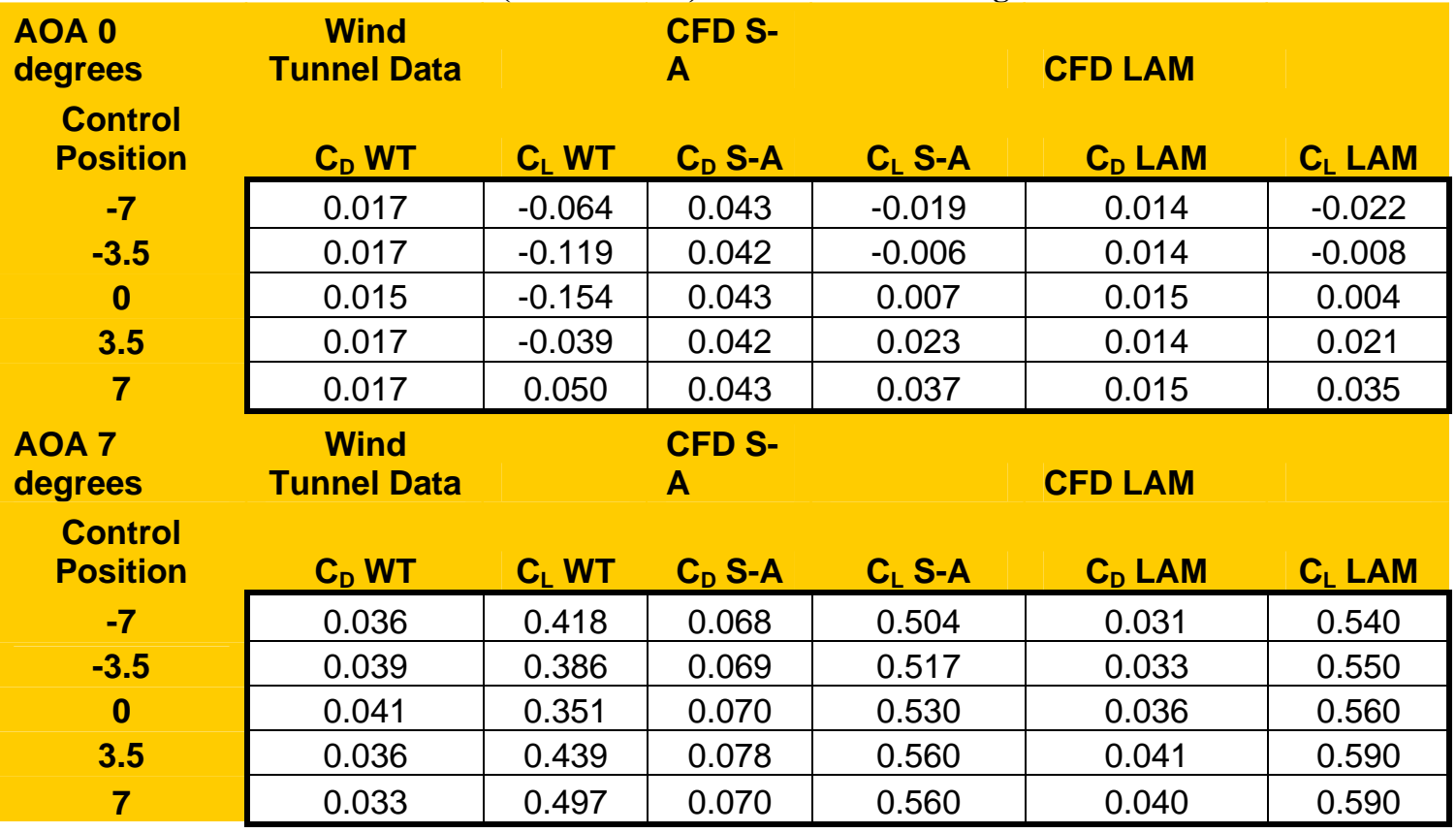

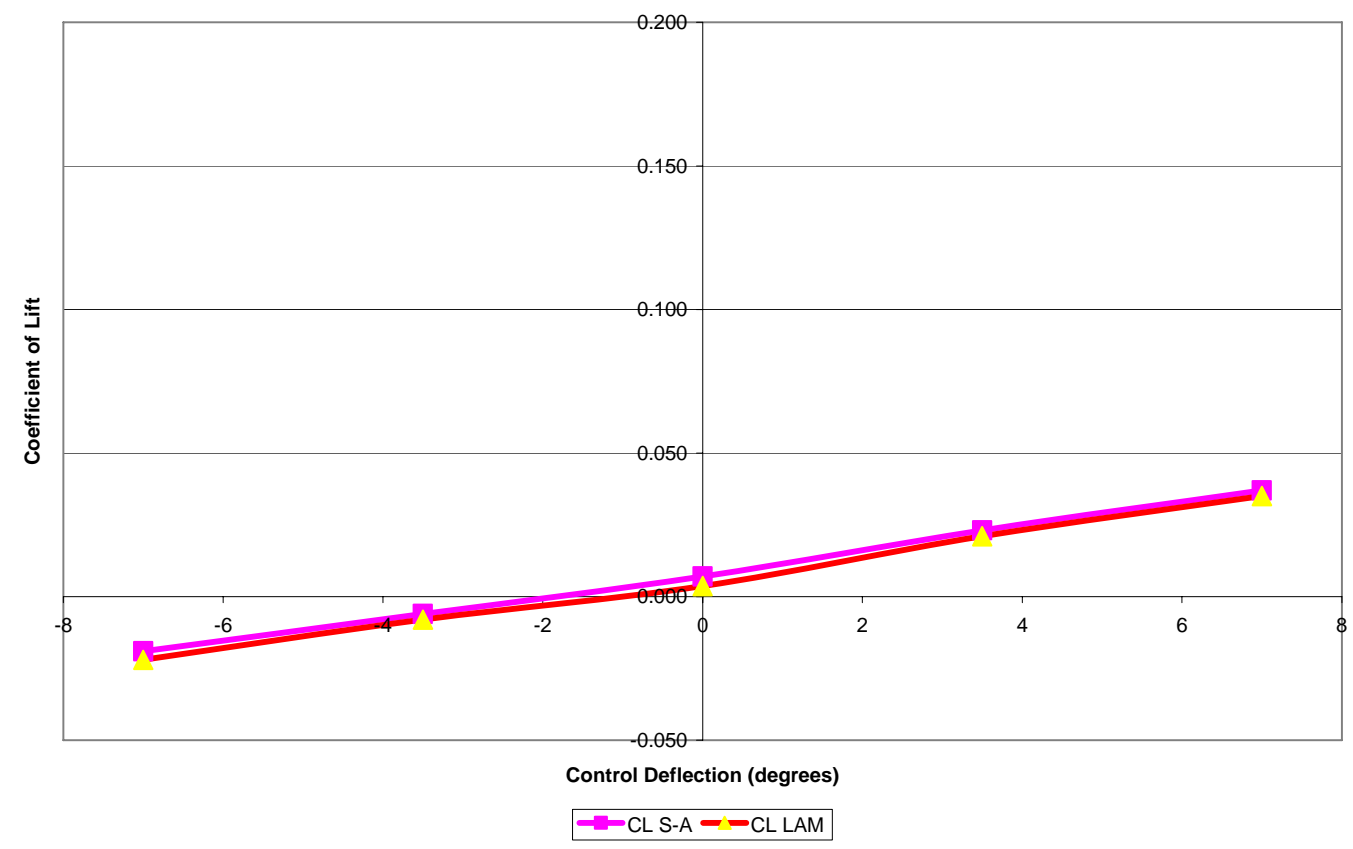

Figure 103 - Coefficient of Lift Comparison between a CFD model using Spalart-Allmaras turbulence model and a Laminar flow CFD model at zero angle of attack. 


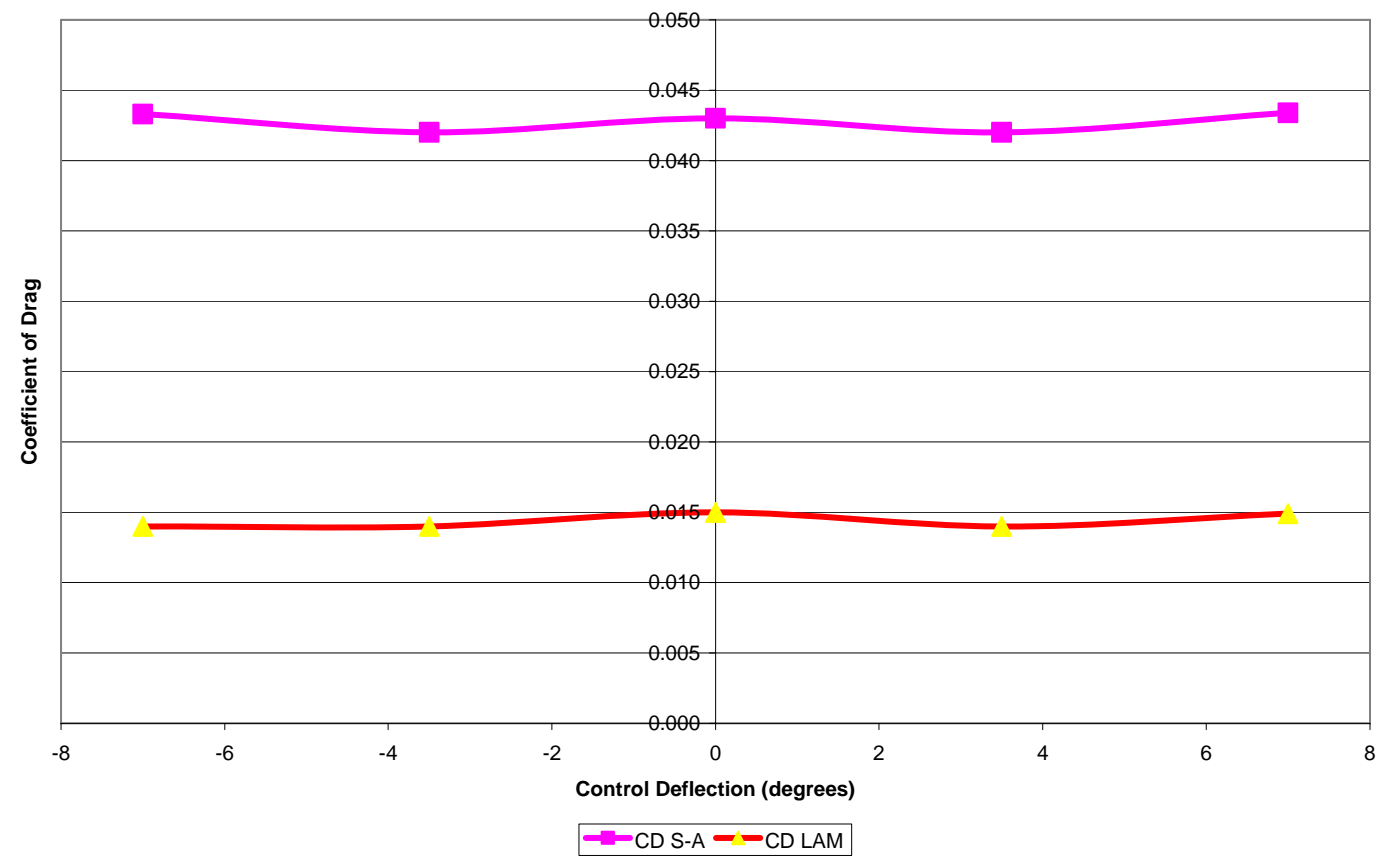

Figure 104 - Coefficient of Drag Comparison between a CFD model using Spalart-Allmaras turbulence model and a Laminar flow CFD model at zero angle of attack.

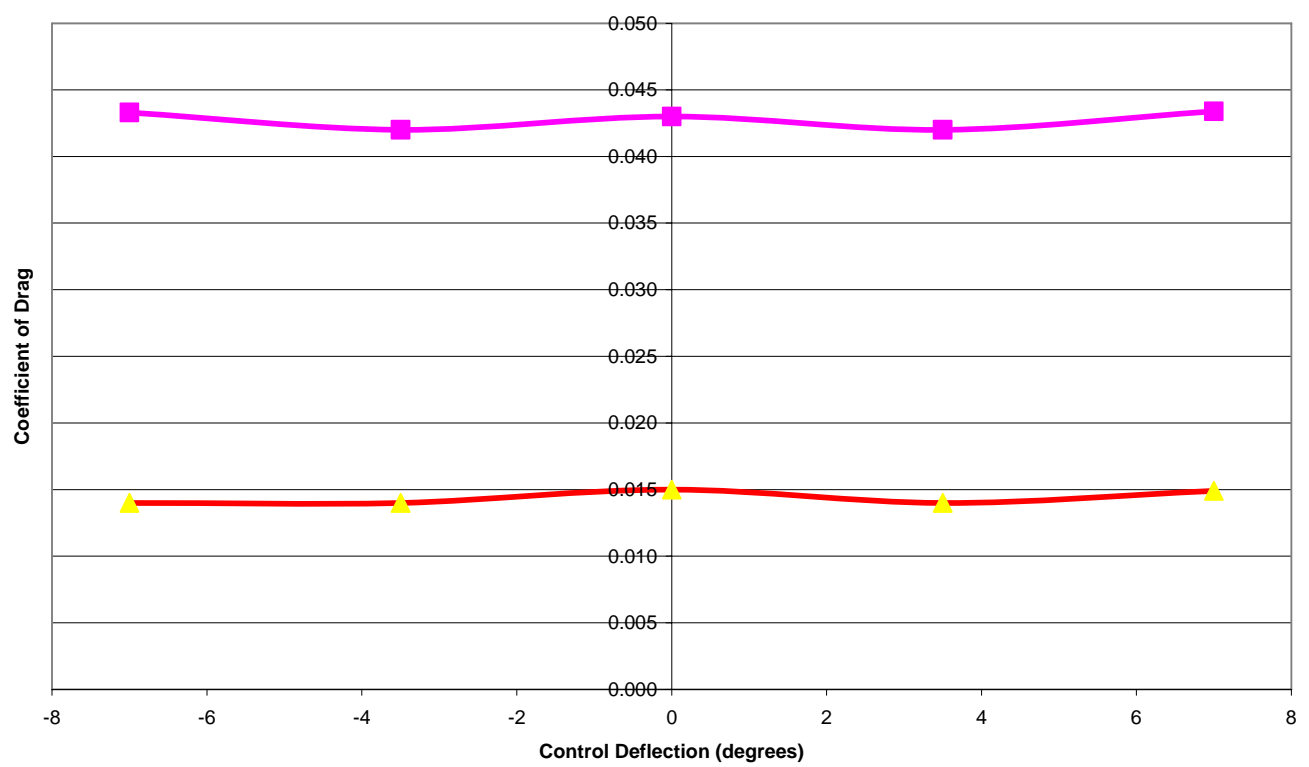

- CD S-A - - CD LAM

.Figure 105- Coefficient of Lift Comparison between a CFD model using Spalart-Allmaras turbulence model and a Laminar flow CFD model at 7 degrees angle of attack. 


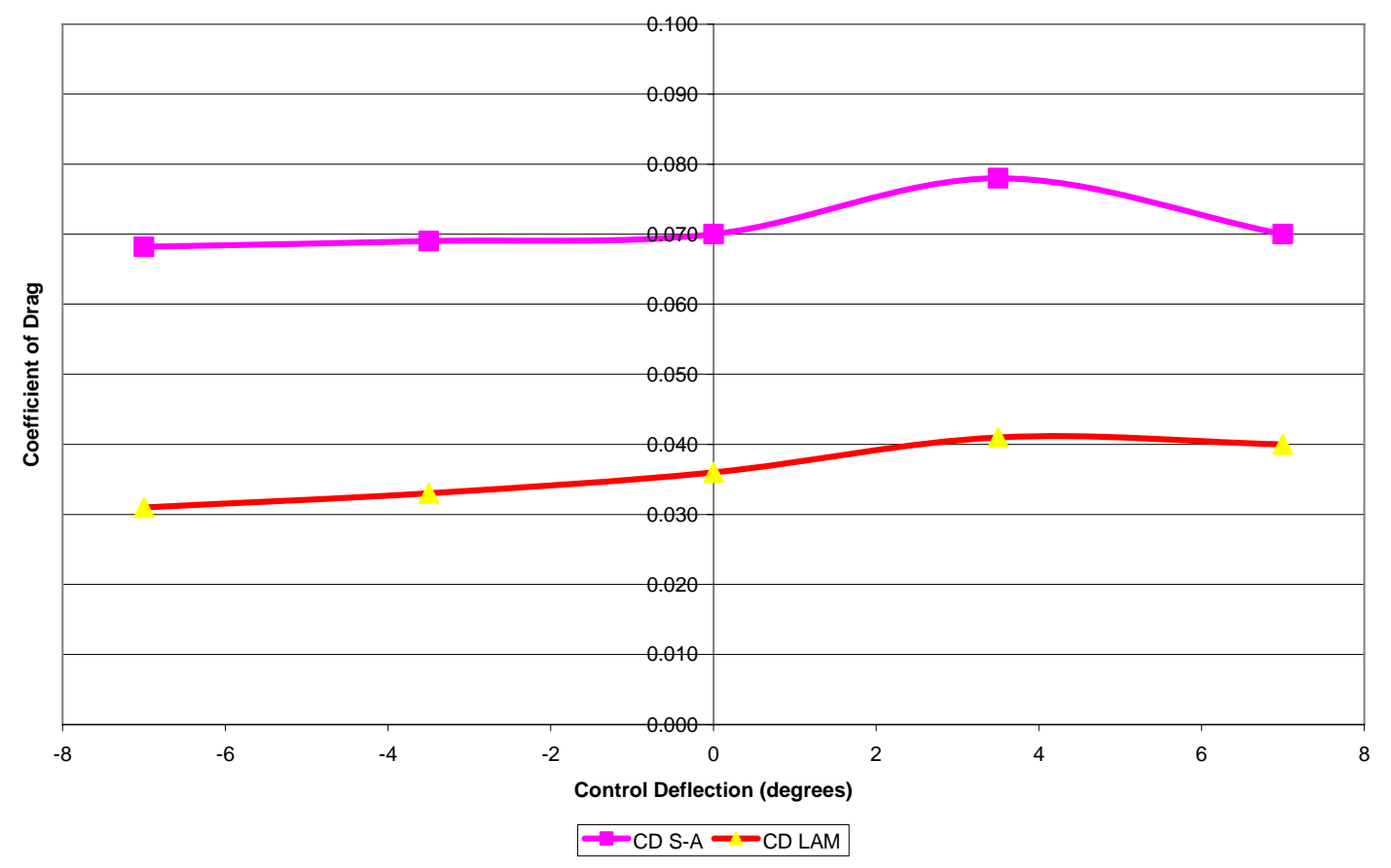

Figure 106 - Coefficient of Drag Comparison between a CFD model using Spalart-Allmaras turbulence model and a Laminar flow CFD model at 7 degrees angle of attack.

In general at zero degrees angle of attack the controls perform much as expected. The lift coefficient of the wing stays around zero. Both the laminar and turbulent models are in good agreement in behavior. Both CFD model's coefficient of drag values are at their highest values at the neutral control position and at the extreme $+/-7$ degree control deflections. The laminar models $C_{D}$ values are lower in magnitude than the turbulent in all conditions. Drag values varied little at zero AOA for any data set. The laminar models varied between 0.014 and 0.017 and the turbulent model varied between 0.042 and 0.043 .

Control behavior is much more interesting when lift is present at 7 degrees AOA. As far as lift goes both data sets show good agreement in magnitude and general behavior. The CFD models show an increase in lift from the -7 degree control deflection through the +7 degree control deflection. The CFD model's $\mathrm{C}_{\mathrm{L}}$ values are between 0.51 to 0.59 . Drag value magnitudes show good agreement between the laminar CFD model and the turbulent data in behavior while the Spalart-Allmaras model showed higher $\mathrm{C}_{\mathrm{D}}$ values. Both models show an increase in $\mathrm{C}_{\mathrm{D}}$ from the -7 degree control position to the neutral control position. Then both the CFD models show $\mathrm{C}_{\mathrm{D}}$ increasing to the +3.5 degree control deflection then heading down again. To aid in the understanding of this data Figure 107 through Figure 108 show pressure and velocity data from the CFD models. 


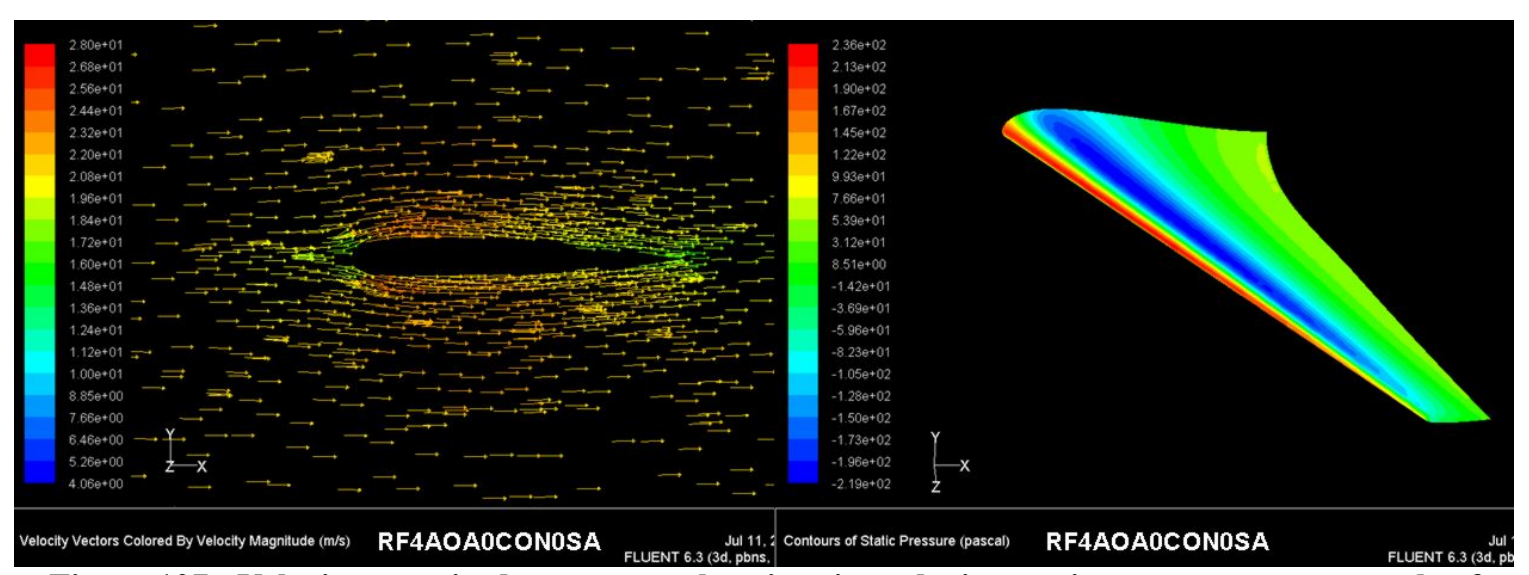

Figure 107 - Velocity magnitude vectors at the wing tip and wing static pressures at zero angle of attack and neutral control position.

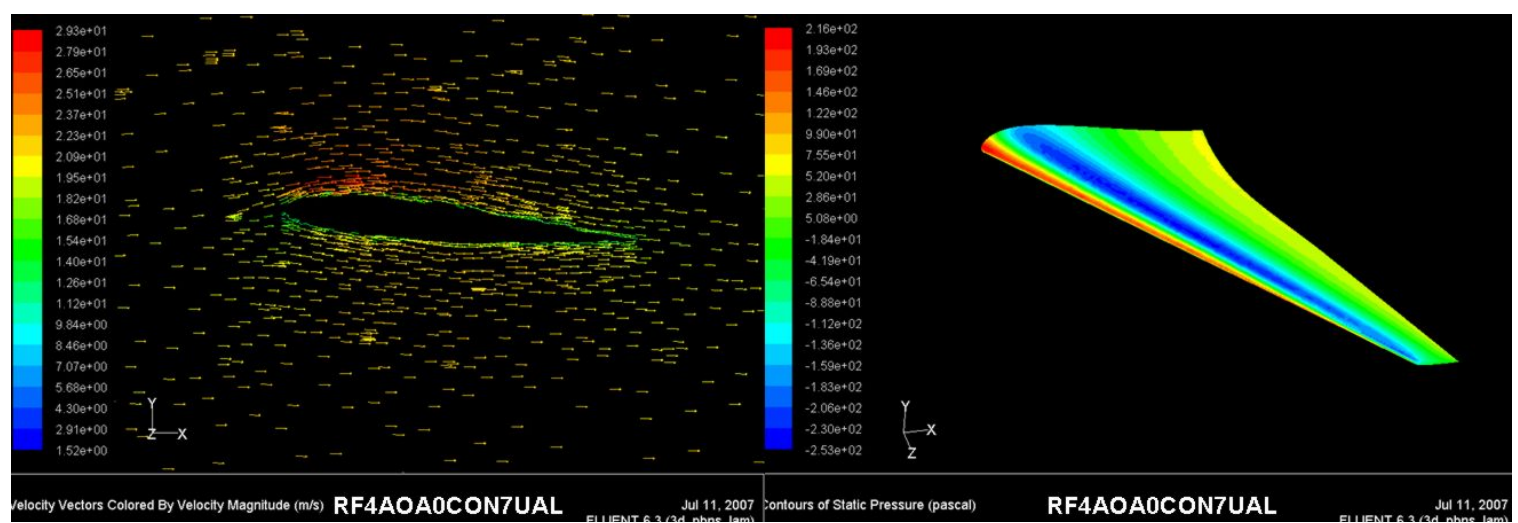

Figure 108 - Velocity magnitude vectors at the wing tip and wing static pressures at zero angle of attack and a 7 degree control deflection.

A region of significant interest is the tip region where control deflections occur. Pressure data from the CFD data shows that very little flow or pressure changes occur in the rootward two thirds of the wing while control deflection are being changed, but there are large changes near the tip. Initially cross sections 2 centimeters inboard of the tip were created to study the flow and pressure field. The tip region is also where most of the differences between the Laminar and the Spalart-Allmaras models occur. At zero degree AOA both the laminar and turbulent models show good agreement in the flow physics. Figure 107 shows the zero AOA,wing pressure field and flow vectors at the tip cross section from the turbulent model. Figure 108 shows the zero AOA,wing pressure field and flow vectors at the tip cross section from the laminar model The seven degree AOA laminar model predicts a region of slow to reversed flow beginning near the root at the trailing edge which extends tip-ward, covering up to half the cord at the tip in the extreme positive control deflections, where as the turbulent model predicts mostly attached flow through all the control positions. The CFD model all show an unusually high upwash at the tip when the wing is at a positive angle of attack. Even when the control deflection in -7 degree the upwash adjusts to give the tip a positive angle of attack. With positive control deflections up to +7 degrees the upwash angle is greater then the control deflection. Figure 109 through Figure 110 show detailed comparisons between various 
cross sections at 3.5 degree and 7 degree control deflections from both laminar and the Spalart-Allmaras models.

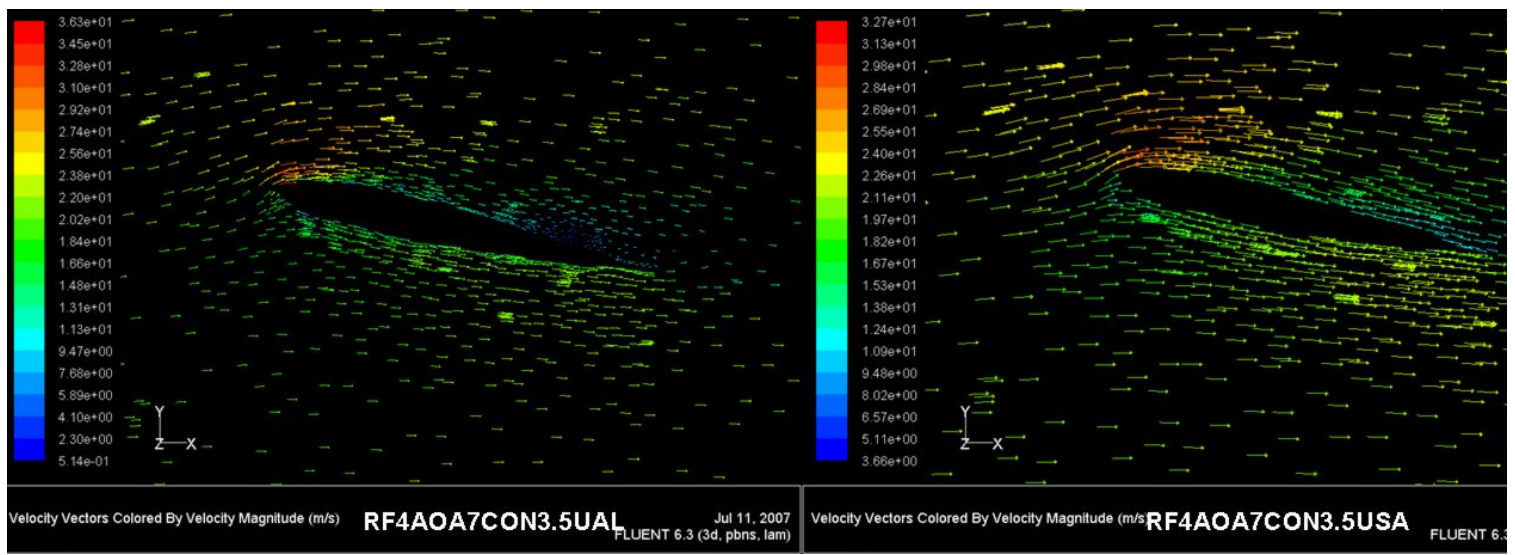

Figure 109 - Velocity magnitude vectors near the wing tip at 7 degrees angle of attack and a 3.5 degree control deflection, both Laminar and Spalart Allmaras models.

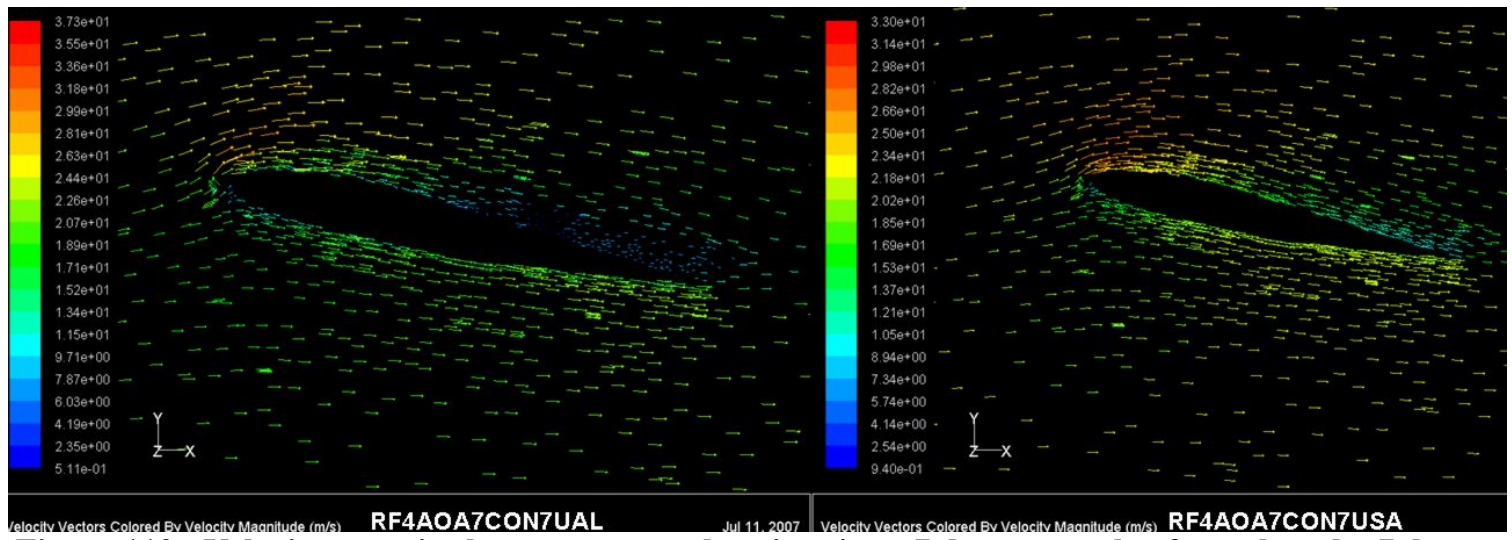

Figure 110 - Velocity magnitude vectors near the wing tip at 7 degrees angle of attack and a 7 degree control deflection, both Laminar and Spalart Allmaras models. 


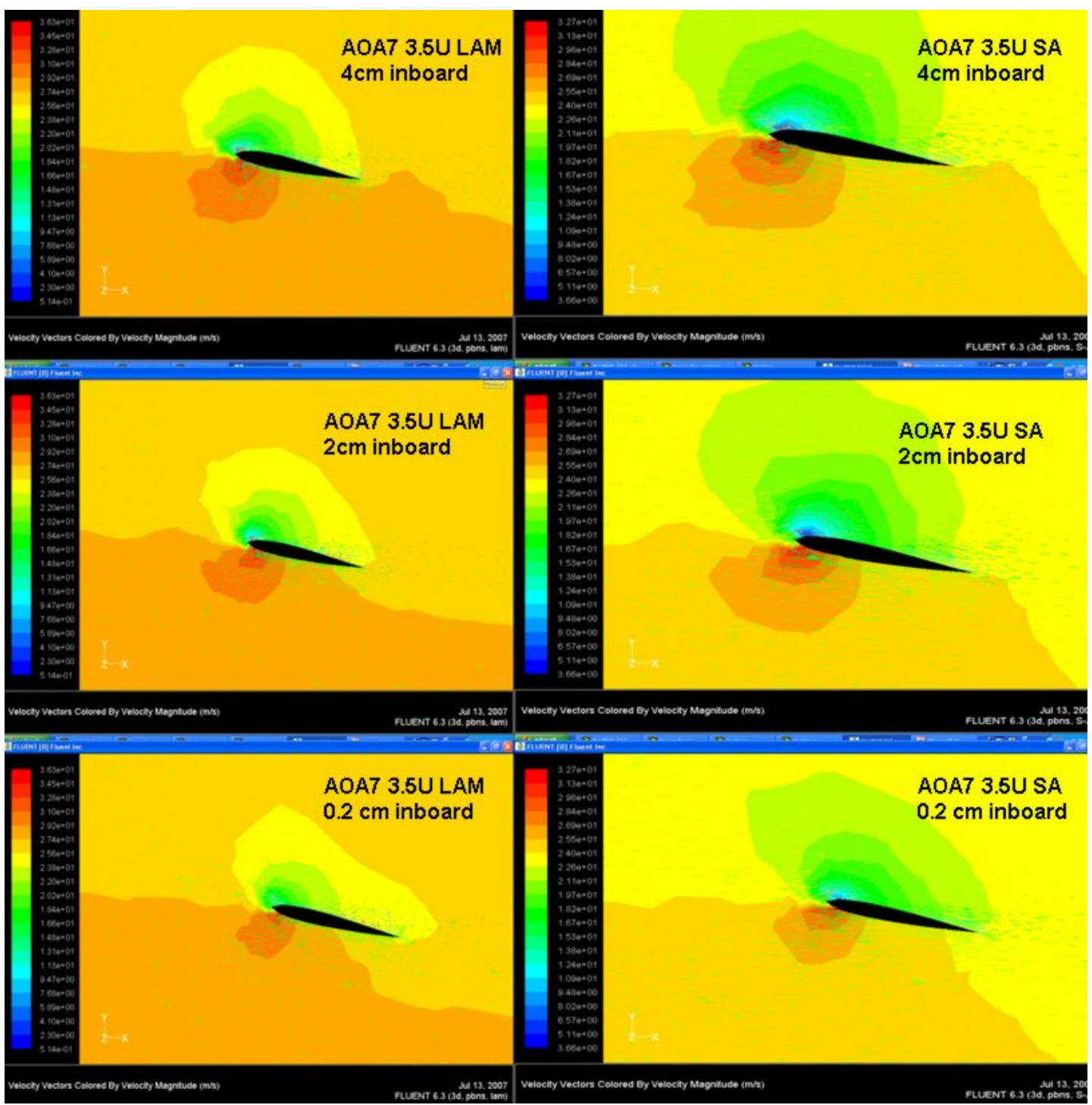

Figure 111 - Static pressures and flow velocity vectors at cross sections in the tip region at 7 degrees angle of attack and a 3.5 degree control deflection, both Laminar and Spalart Allmaras models, $2 \mathrm{~cm}$ (distance fromtip), $7 \mathrm{U}$ (7 degrees up, LAM (laminar), SA (Spalart-Allmaras).

Figure 112 through Figure 121 show the static pressure on the wings top and bottom surfaces at the five test control positions while the wing is at a 7 degree angle of attack. At the -7 degree control deflection there is a pod of low pressure air which extends from 2 inches from the root out $2 / 3$ rds of the wing along about the $10 \%$ cord. There is a corresponding high pressure pod on the lower wing surface directly below the leading edge which also runs out $2 / 3$ rds of the wing and gently tapers toward the leading edge of the tip. At the negative 3.5 degree control deflection the patters are similar except both the high and low pressure pods of air extend another $10 \%$ toward the tip. At the 0 degrees control position both the high and low pressure pods extend to approximately 2 inches from the tip and both gently taper to the tip leading edge. At the +3.5 degree control deflection the low pressure pod extends to the tip and intensifies approximately 60 pascals and begins to rotate toward the leading edge. The high pressure air on the bottom 
of the wing extends to cover $1 / 3$ of the tip. These trends continue as the control is moved to the +7 degree control deflection. The low pressure area intensifies another 60 Pascal and now has moved to cover the leading edge of the outboard $1 / 3$ of the wing. The pressure pod on the lower wing surface again has a gentle taper to the tip leading edge. In general, the most dramatic pressure changes occur around the +3.5 degree control deflection.

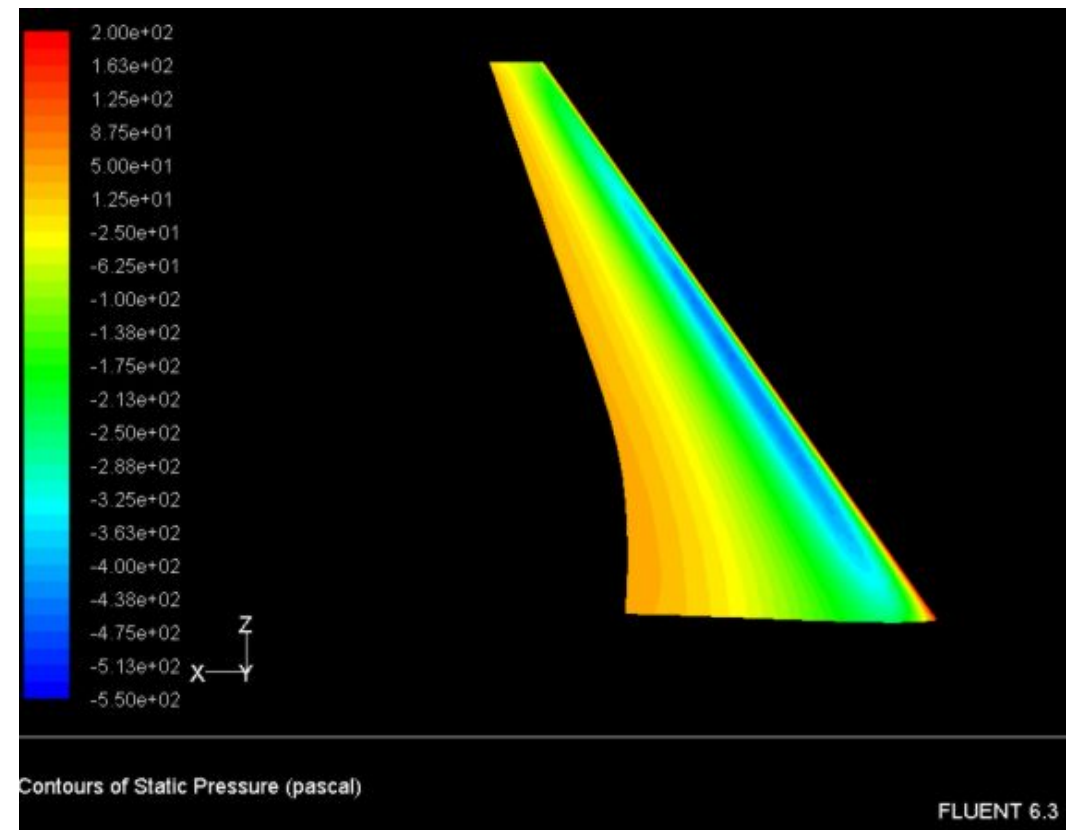

Figure 112 - Static pressures on the wing top at 7 degrees angle of attack and a -7 degree control deflection.

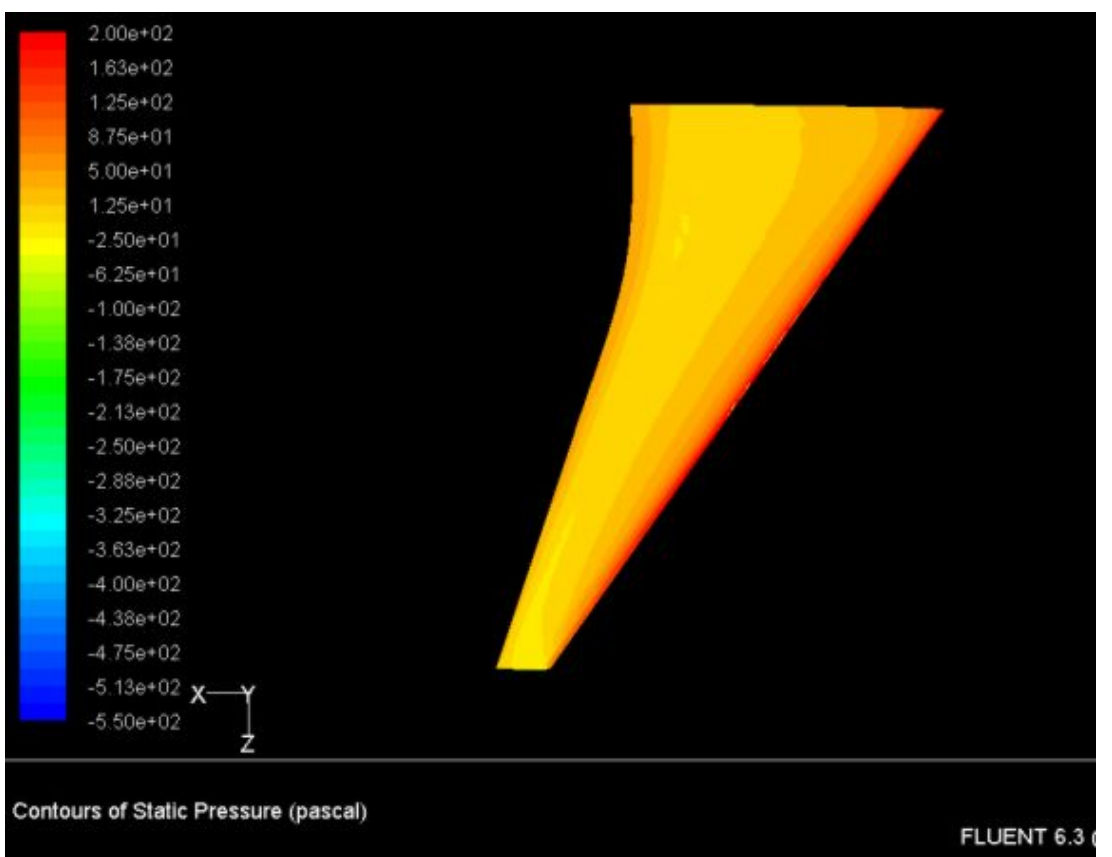

Figure 113 - Static pressures on the wing bottom at 7 degrees angle of attack and a -7 degree control deflection. 


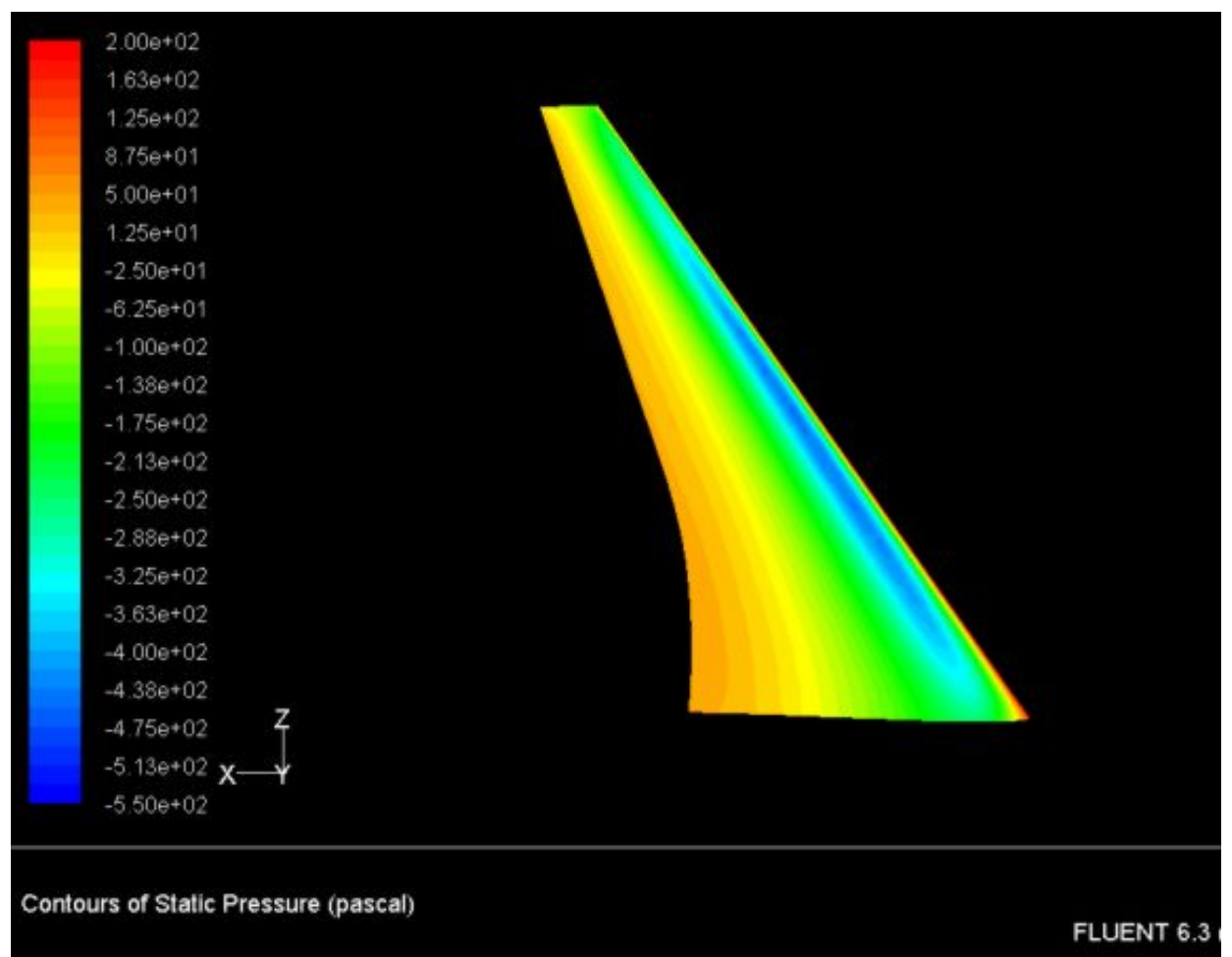

Figure 114 - Static pressures on the wing top at 7 degrees angle of attack and a $\mathbf{- 3 . 5}$ degree control deflection.

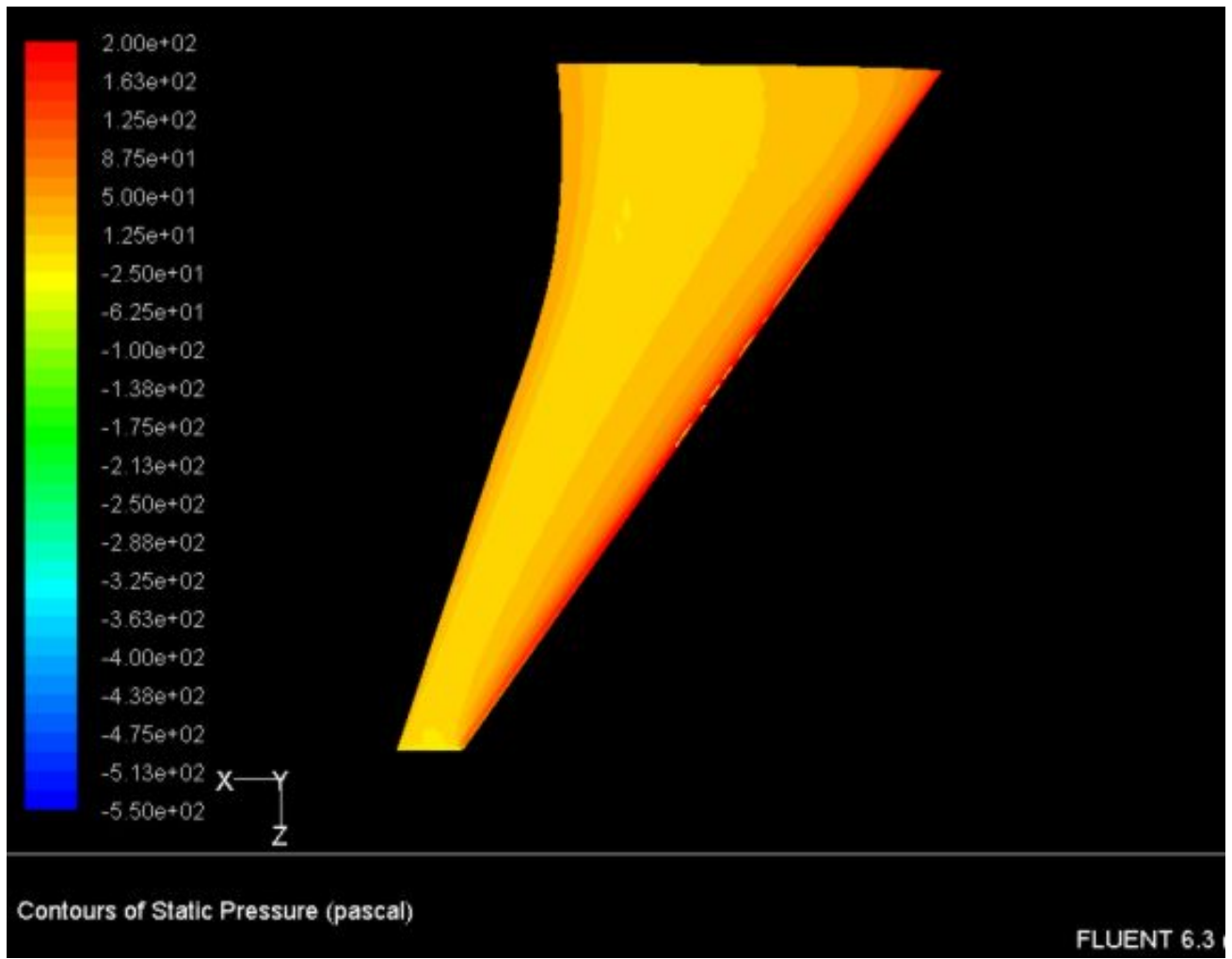

Figure 115 - Static pressures on the wing bottom at 7 degrees angle of attack and a -3.5 degree control deflection. 


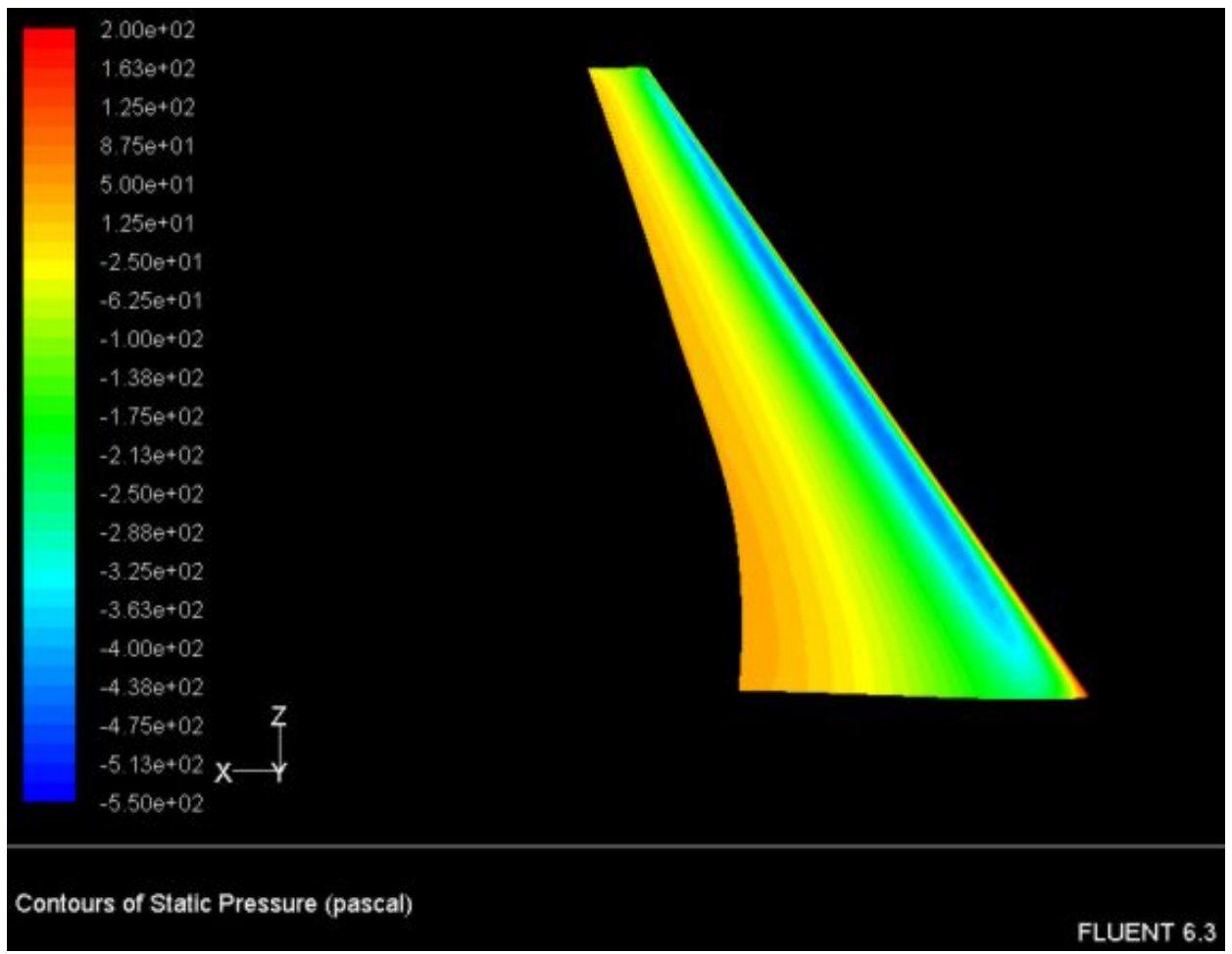

Figure 116 - Static pressures on the wing top at 7 degrees angle of attack and a 0 degree control deflection.

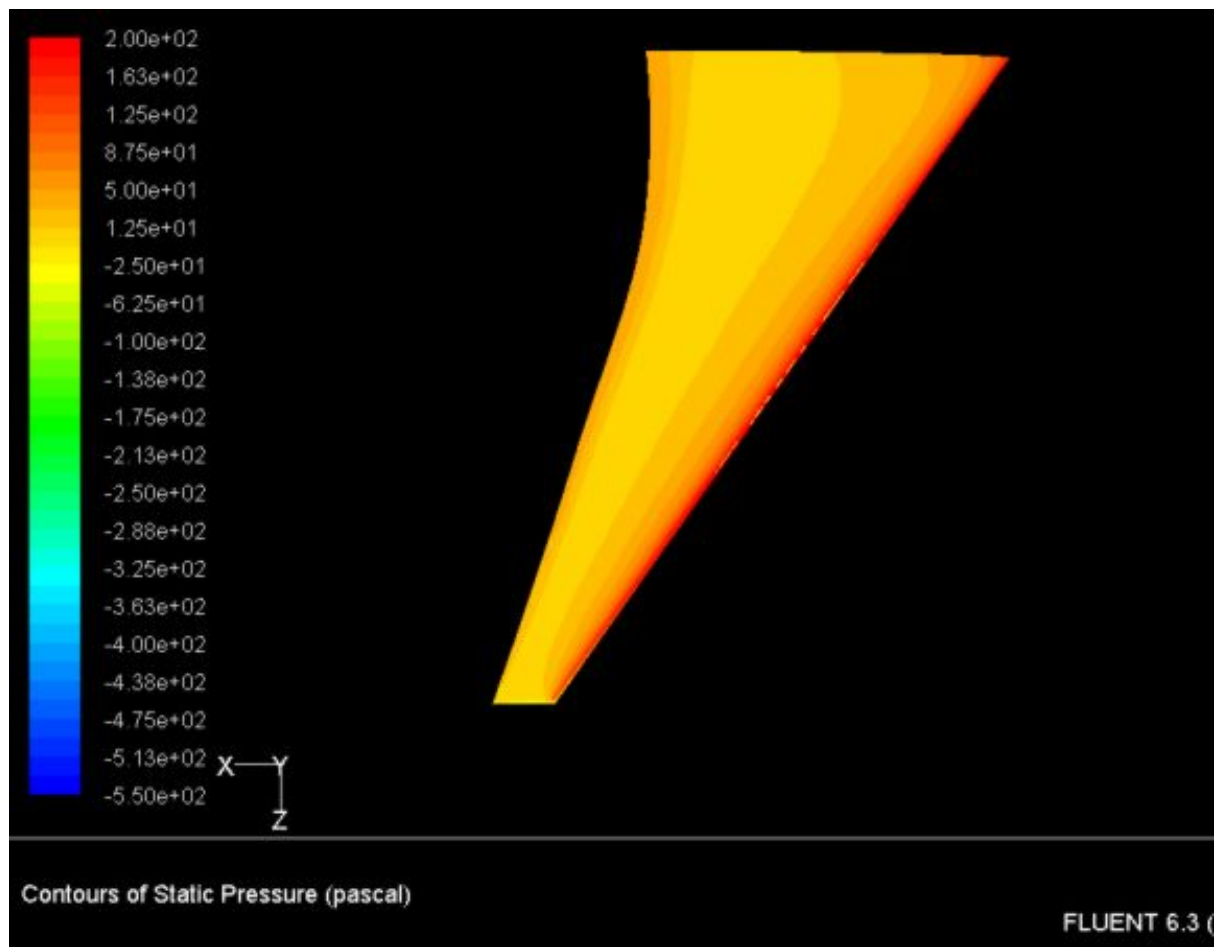

Figure 117 - Static pressures on the wing bottom at 7 degrees angle of attack and a 0 degree control deflection. 


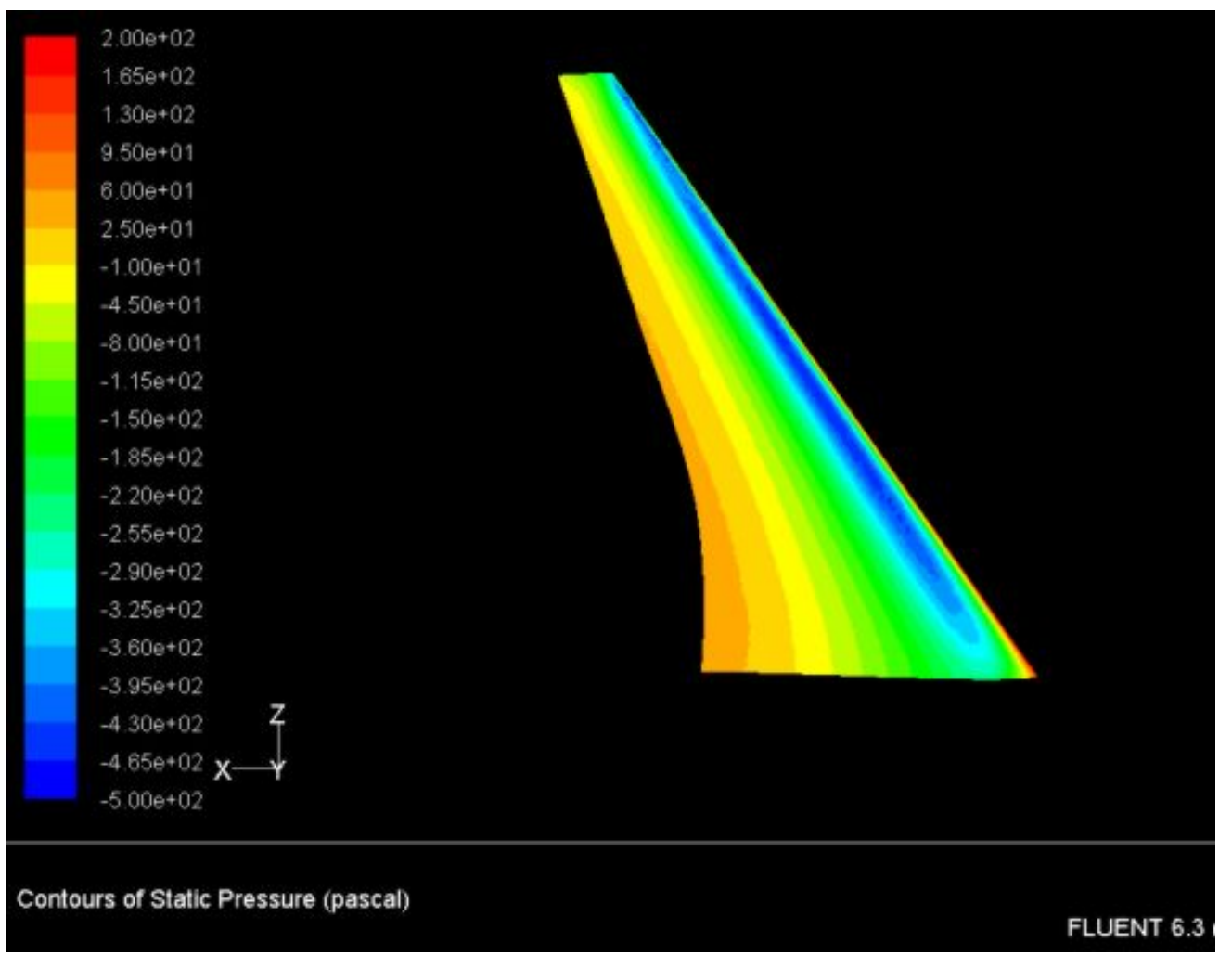

Figure 118 - Static pressures on the wing top at 7 degrees angle of attack and a +3.5 degree control deflection.

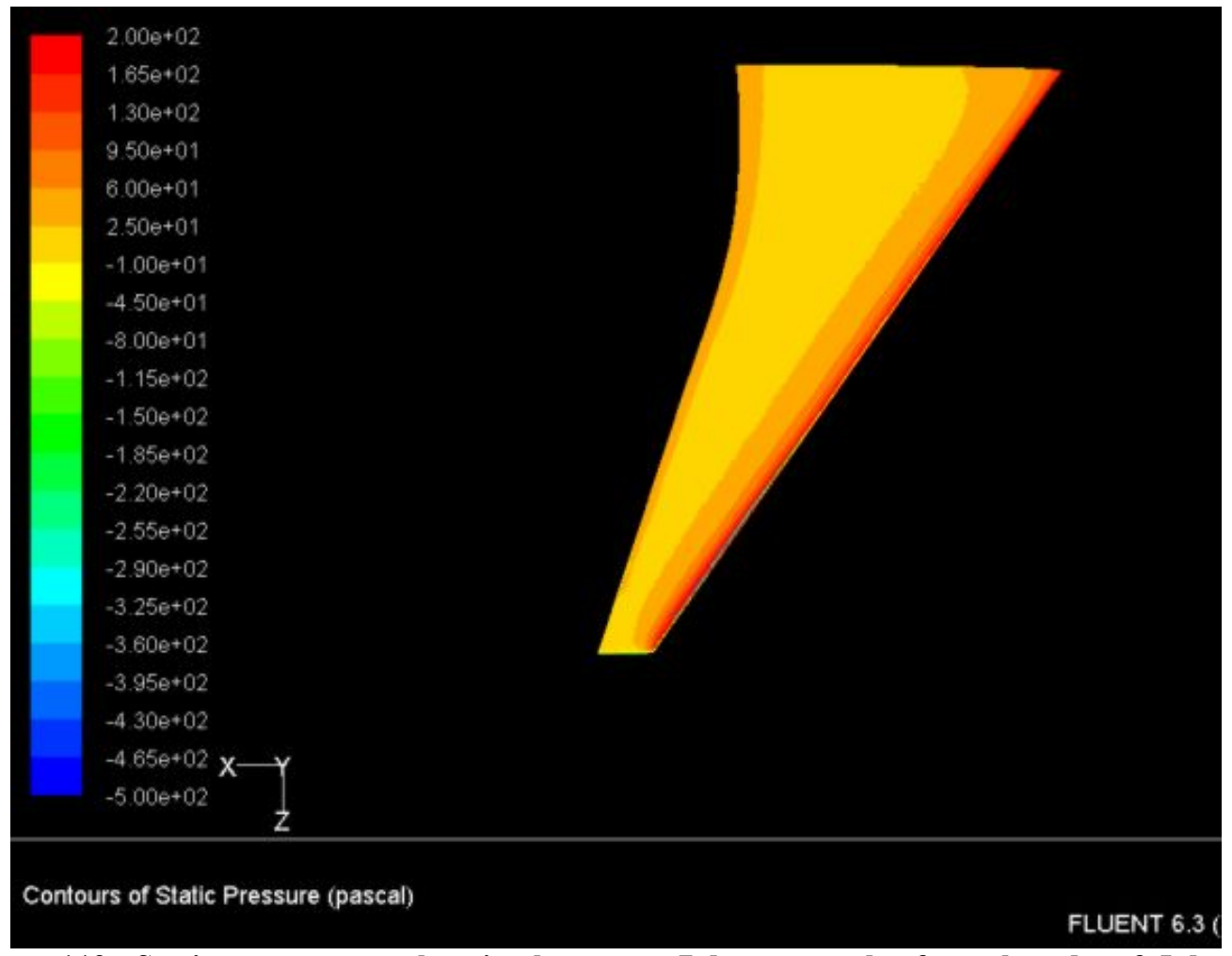

Figure 119 - Static pressures on the wing bottom at 7 degrees angle of attack and a +3.5 degree control deflection. 


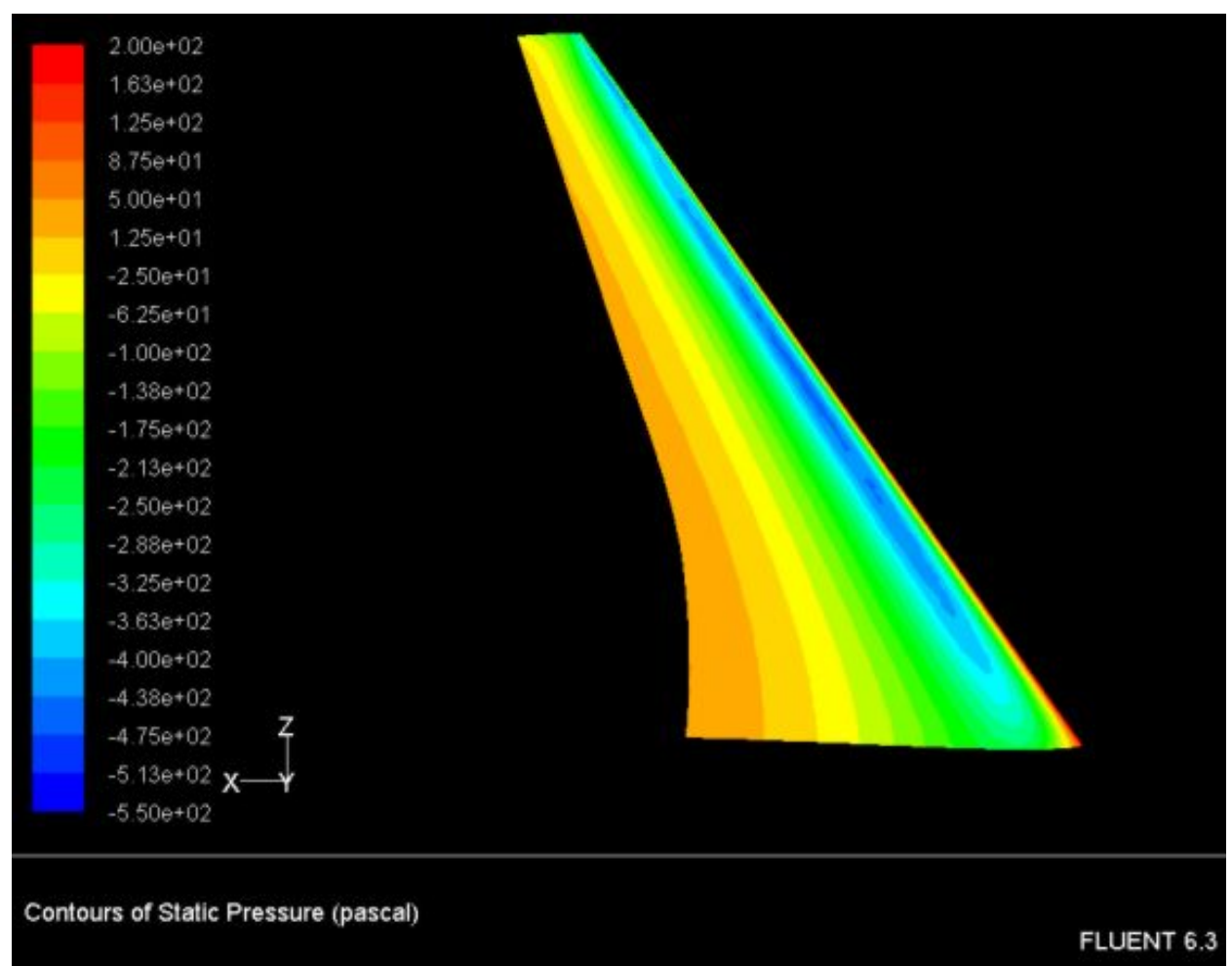

Figure 120 - Static pressures on the wing top at 7 degrees angle of attack and a +7 degree control deflection.

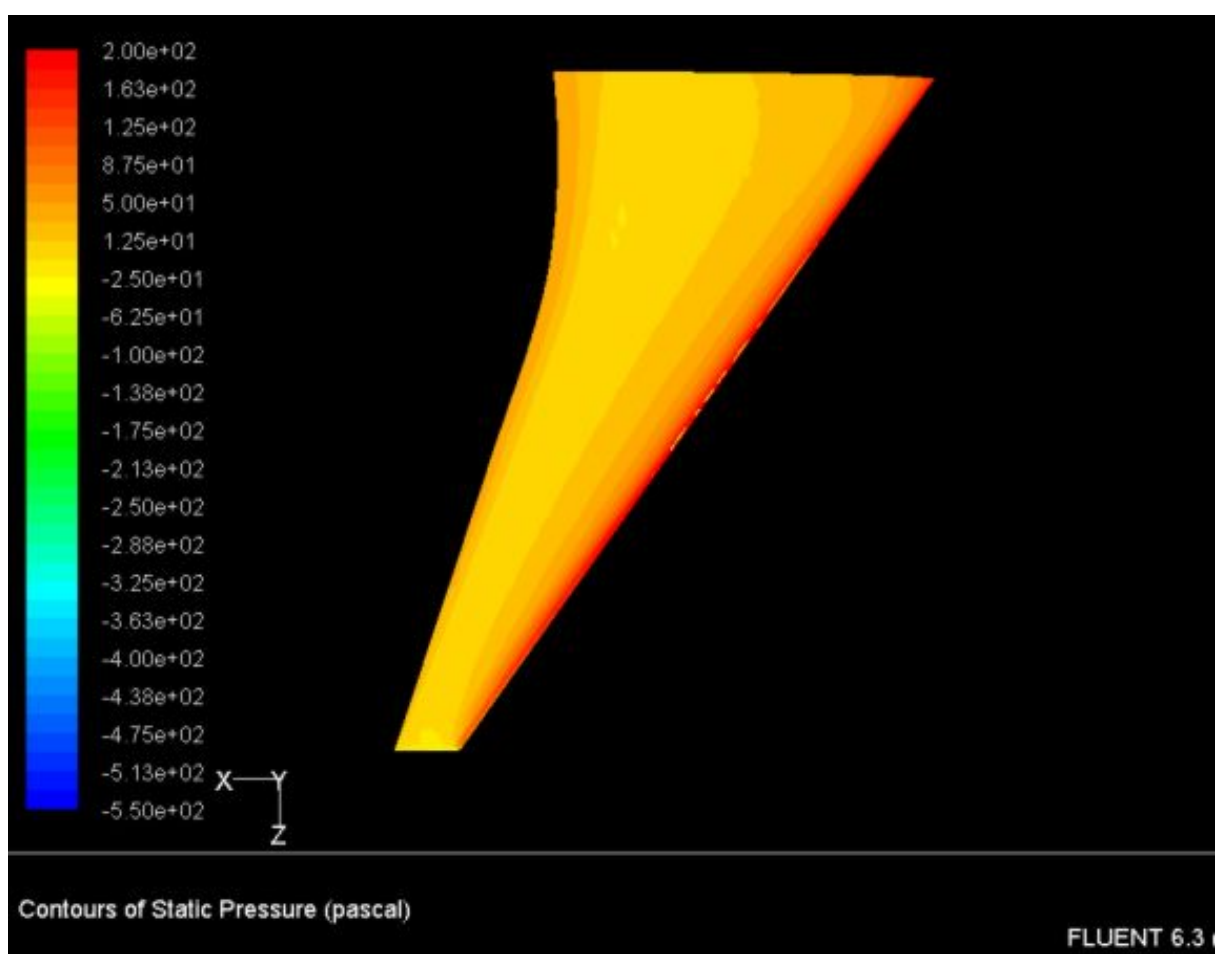

Figure 121 - Static pressures on the wing bottom at 7 degrees angle of attack and a +7 degree control deflection. 
To facilitate the study of wake flow and pressure structures planes (Figure 122) were generated perpendicular to the wind tunnel walls and the root. Only subtle pressure differences due to down wash are visible out beyond approximately 2 inches downstream from the tip trailing edge. (Figure 123) Closer to the tip low pressures indicative of the core of a tip vortex are visible. This low pressure core structure intensifies from the -7 degree control deflection until the +3.5 degree control deflection and then appears to breakup rapidly as the control is moved to +7 degrees. These core structures can be seen in cross sections passing through the aft 1 inch of the wing tip at all 5 control positions in Figure 124 through Figure 126.

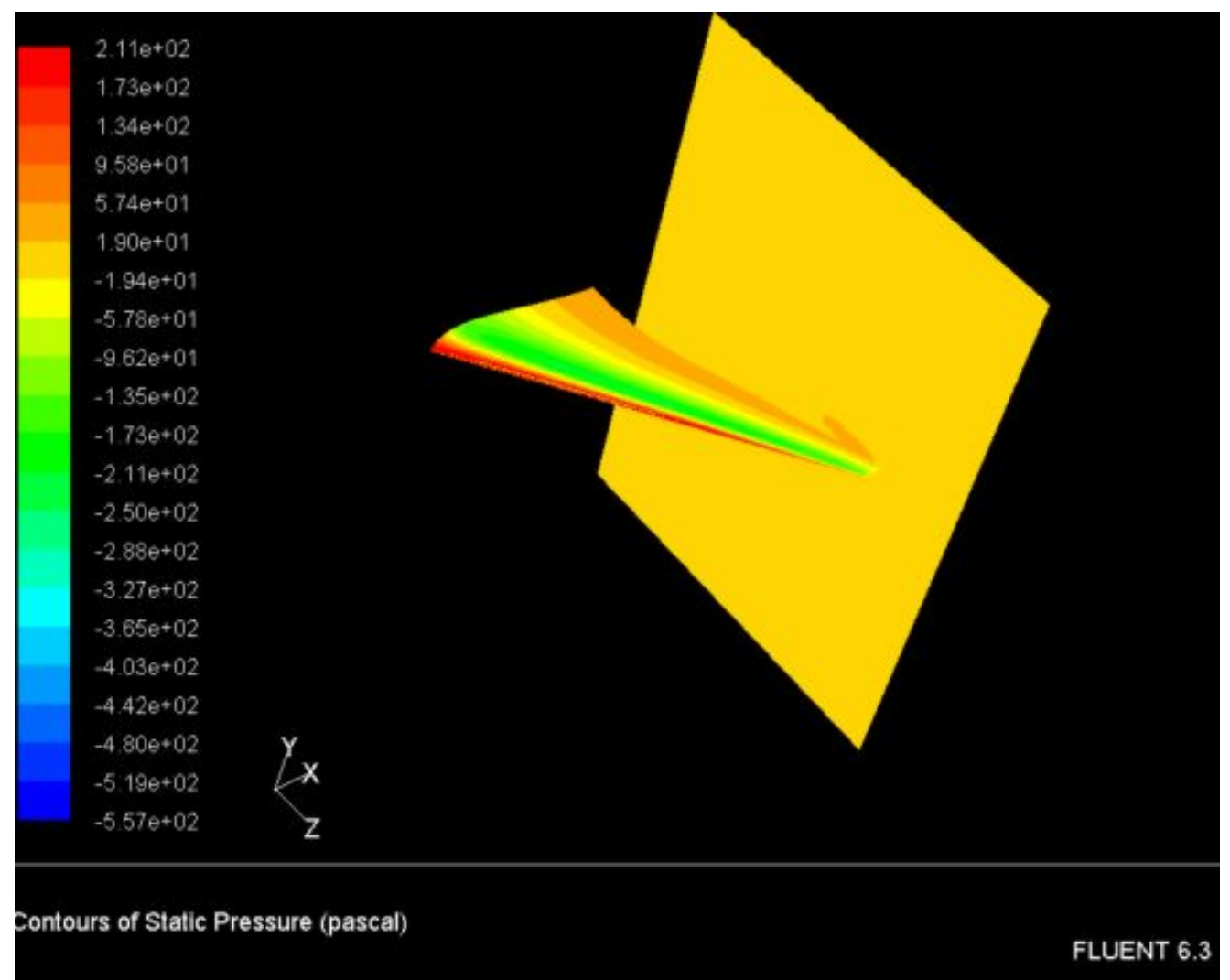

Figure 122 - Example of one of the planes generated to study the wake pressures and flows.

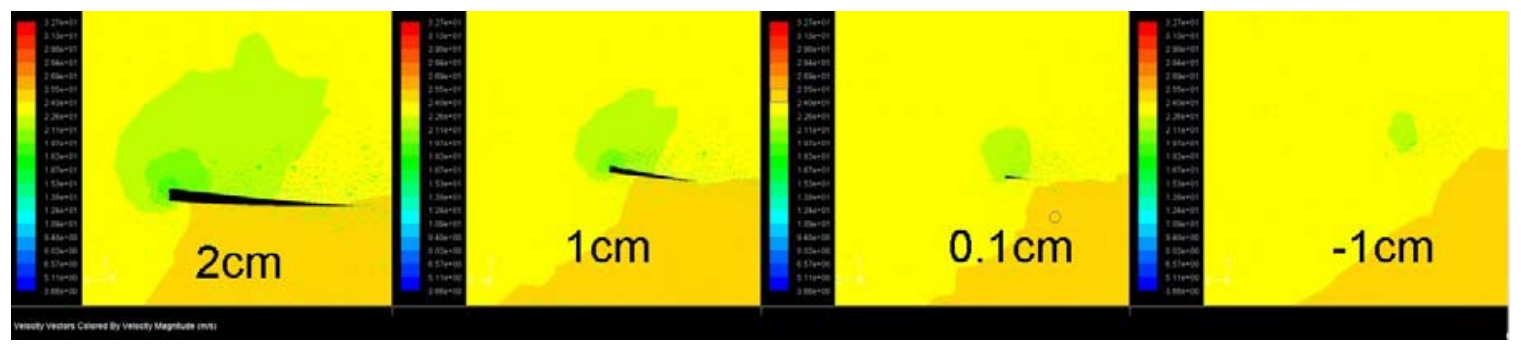

Figure 123 - Typical rapid dissipation of low pressure core structure down stream of the tip. By 1 inch behind the tip trailing edges the structure has mostly disappeared. 


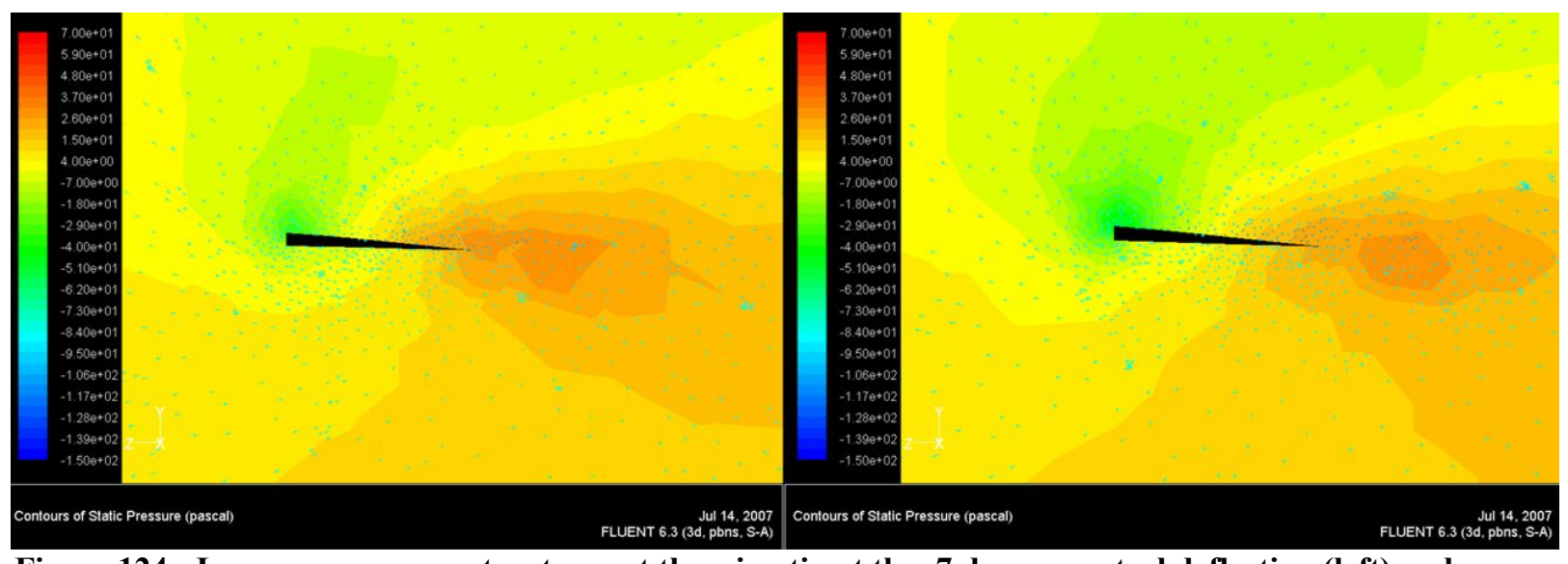

Figure 124 - Low pressure core structures at the wing tip at the -7 degree control deflection (left) and the -3.5 control deflection (right).

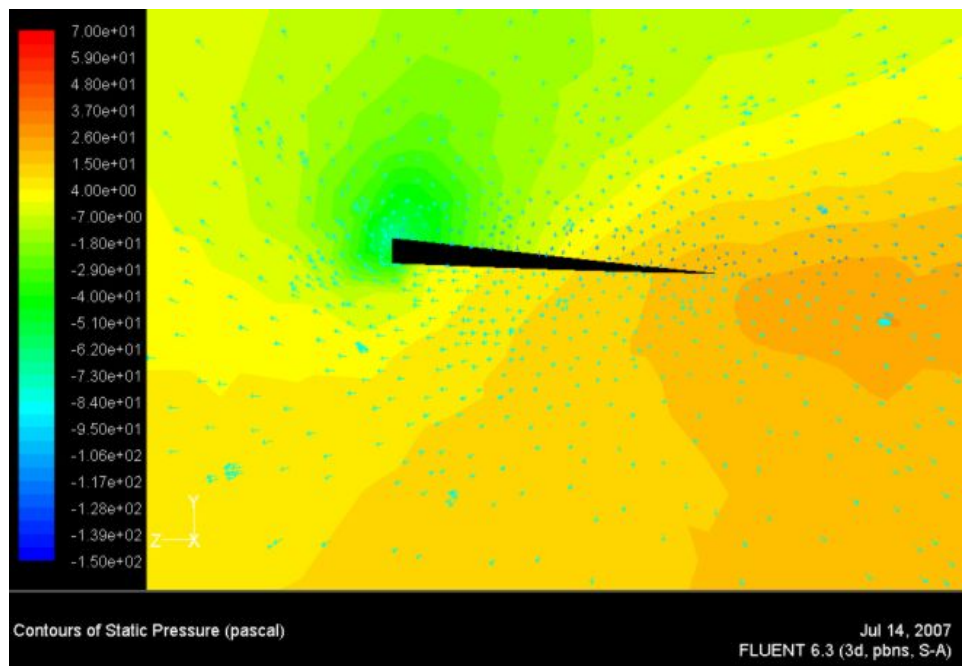

Figure 125 - Low pressure core structures at the wing tip at the zero degree control deflection.

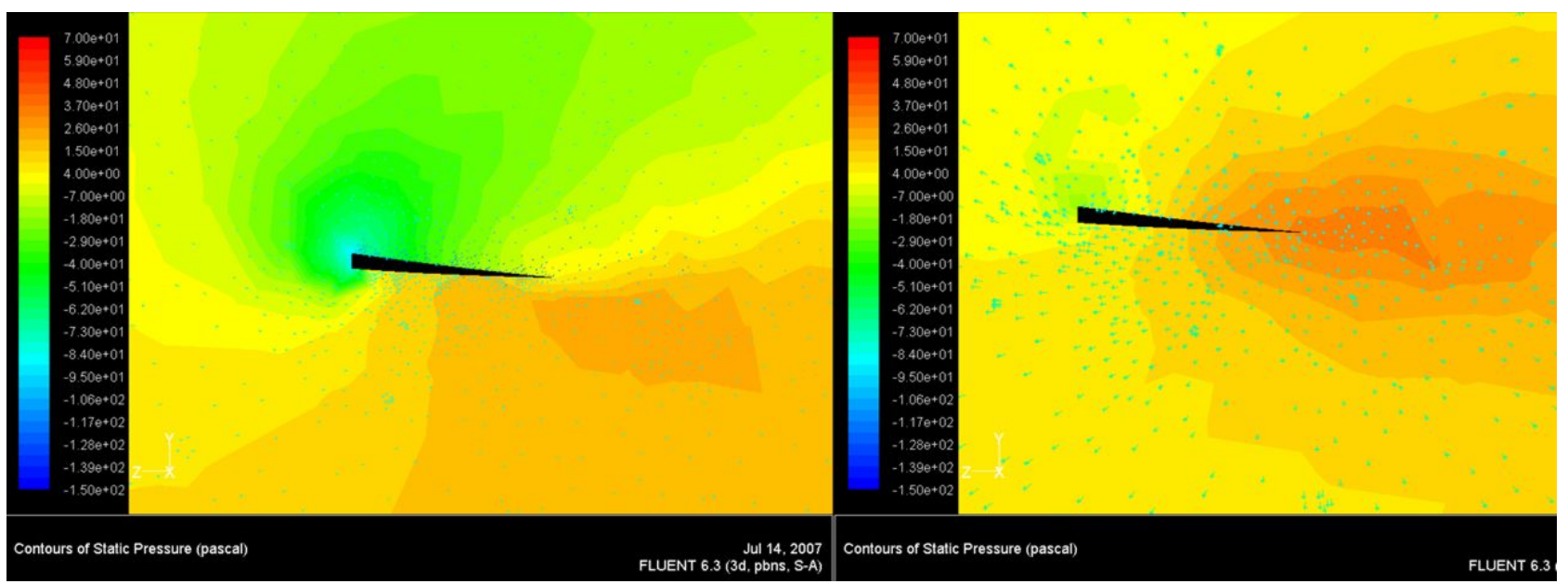

Figure 126 - Low pressure core structures at the wing tip at the +3.5 degree control deflection (left) and the +7 control deflection (right). 


\section{Flight testing of the adaptive washout morphing mechanism}

In order to show the feasibility of the WVU wing morphing mechanism design a flight demonstrator was designed. The flight demonstrator design began with the wind tunnel morphing model as the initial design. The size of the demonstrator was determined based on the following factors: Cost, time for construction and proper wing load with data acquisition system on board. To minimize cost and construction time the minimum size aircraft was desired while still being in similar Reynolds number range as the wind tunnel tests. The combined weight of the data acquisition system and instruments is approximately $4 \mathrm{oz}$. A study of tailless aircraft wing loading was conducted, the results of which are shown in Figure 127.

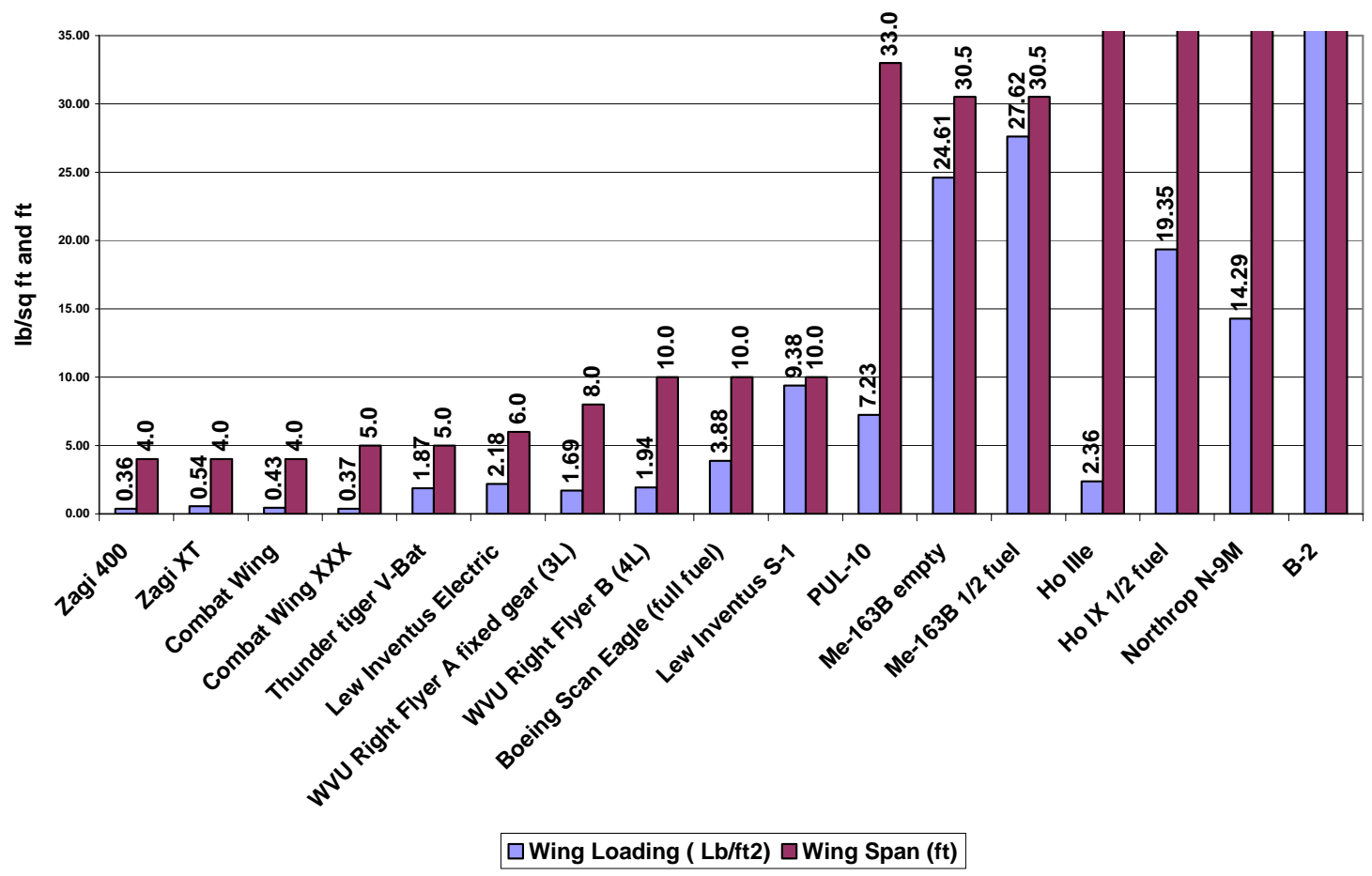

Figure 127 - Results of wing loading study of production sweptwing tailless aircraft.

The wing loading study and test glider experience lead to the determination that the wing loading should be below $1.3 \mathrm{lbs} / \mathrm{ft}^{2}$, so the aircraft should have a wing area of at least 7 square feet considering structural and propulsion weight. One of the previous WVU tailless flight demonstrators had a span of 7 feet, an area of 7.962 square feet and the PUL-10 type elevons. The experience with this aircraft and the ability to compare the flight characteristics lead to the decision to make the new morphing flight demonstrator have a 7 foot span. The additional wing area provided by the $7 \mathrm{ft}$ span also makes it possible to either add additional sensors and computer equipment or additional batteries for improved endurance. 
A 3D model was created in Solid Works which had the same geometry as the wind tunnel model except had a $7 \mathrm{ft}$ span. Weight is a critical factor in this aircraft because it will use a unproven control system so the lightest structure possible within cost constraints was developed. A full female mold with composite materials provided by Aurora Flight Sciences was constructed to a $+/-0.040$ inch tolerance from the CAD model. A leading edge cuff was developed from layups off of the original WVU tailless flight demonstrator. The cuff forms a "C" spar along the full leading edge of the wing back to the $20 \%$ cord location on both the top and bottom wing surfaces. The "C" spar was constructed from 1 ply of $3 \mathrm{k}$ carbon unidirectional fabric sandwiched between a single layer of $5.7 \mathrm{oz} 120$ style carbon fabric and layer of $0.6 \mathrm{oz}$ E-glass fabric (for surface finish). The top and bottom skins are constructed from $1 / 8$ " Rohacell rigid aerospace core foam encased with $2 \mathrm{oz} 120$ style E-glass fabric on the exterior surface and on the interior surface. Six 1/16 inch fiberglass covered balsa ribs are built into the skins by notching into the lower skin core and with core pan-downs in the upper skin. The "C" spar was release taped into the skin molds in order to form a bonding joggle for later assembly. (Figure 128 and Figure 129)

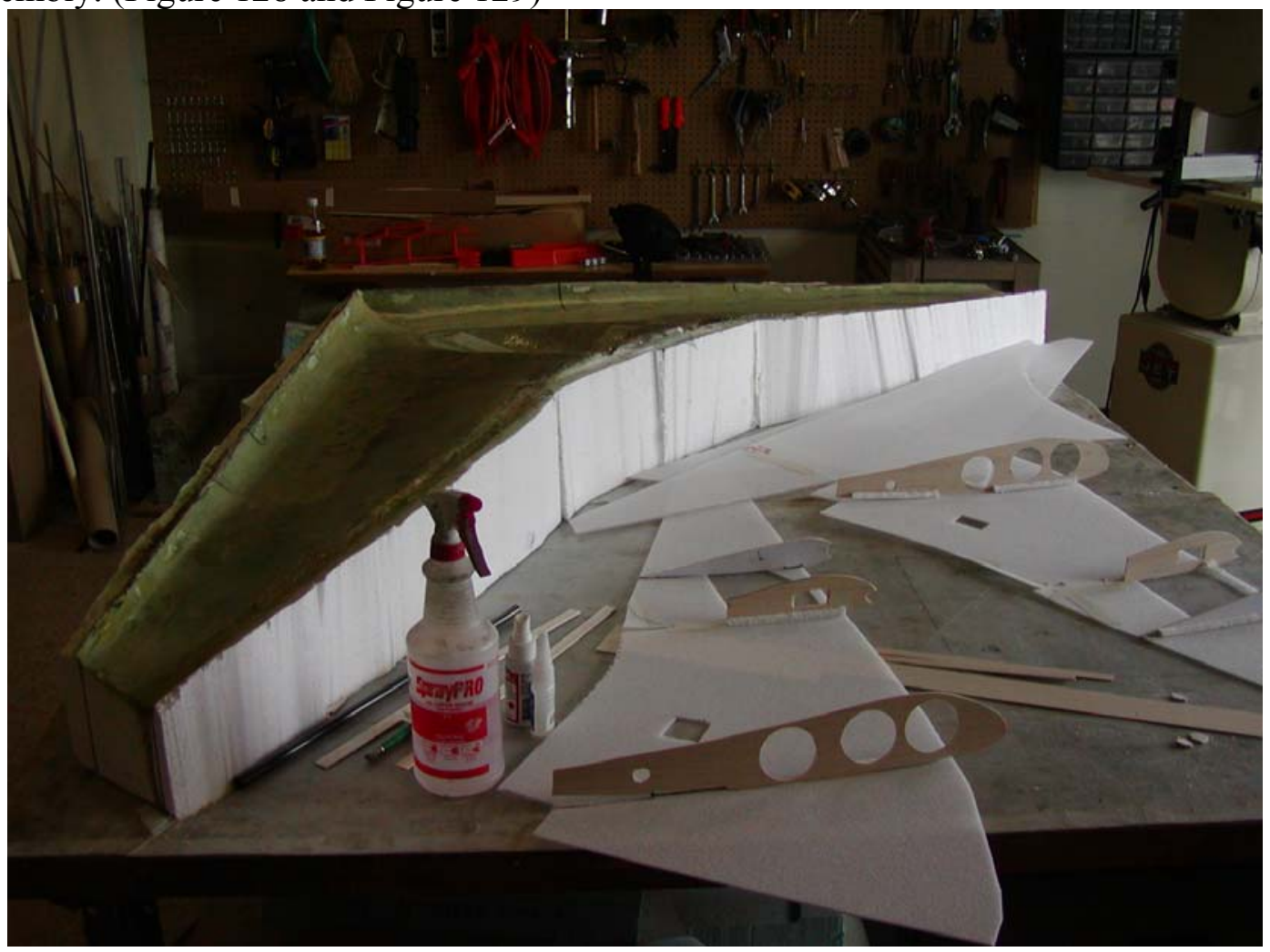

Figure 128 - Full scale lower skin mold with Rohacell cores and glass/balsa ribs in foreground. 


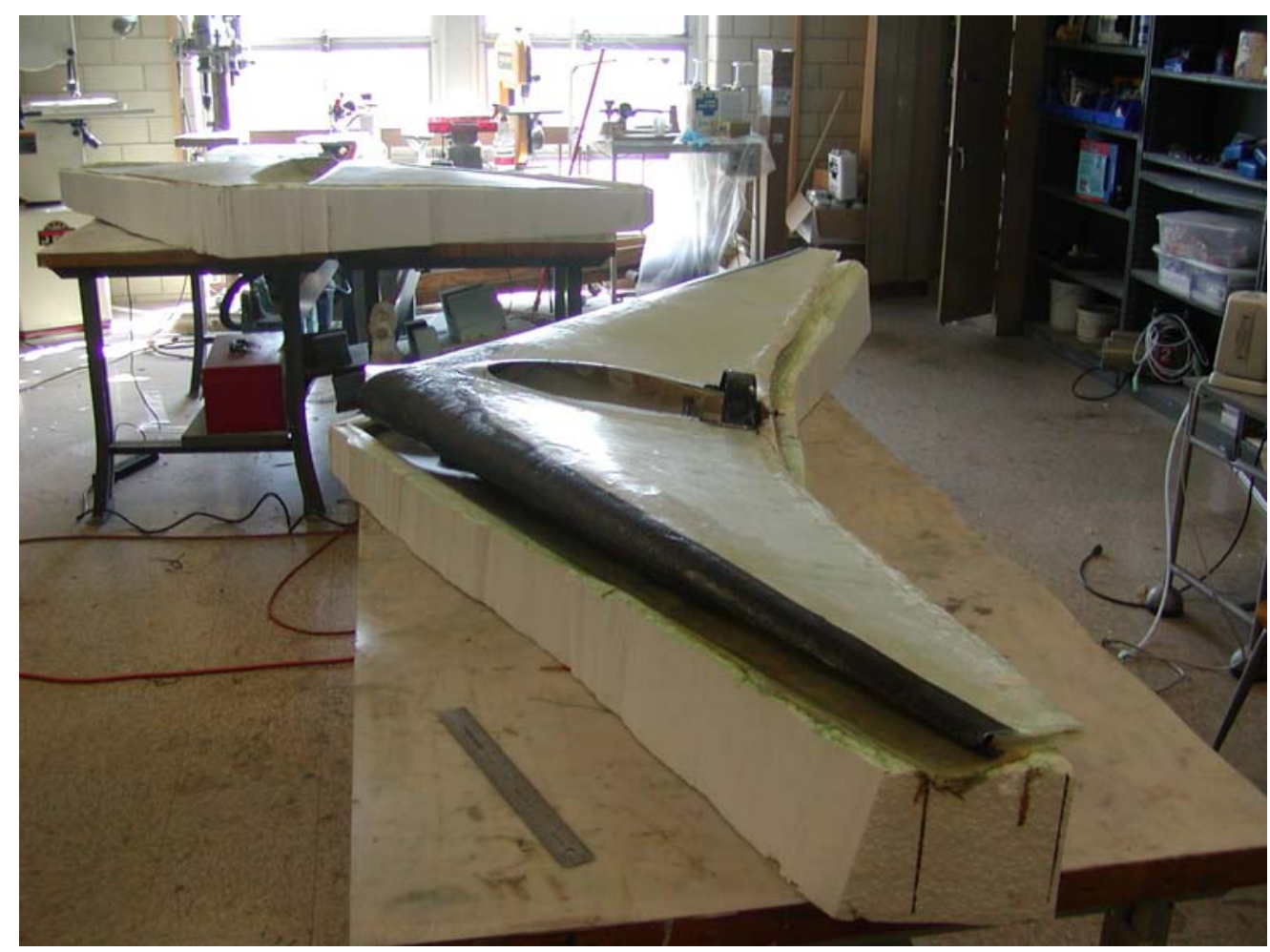

Figure 129 - Female molds with the upper skin bonded to the carbon cuff.

All internal electronics, actuators and structures were installed while the skins were separated. After completion of internals the lower skin was bonded and riveted to the upper skin and cuff.

The original morphing mechanism design was modified to decrease weight and to increase the rigidity. The lessons from the earlier models indicated that the leading edge torque rod needs to be as large a diameter as possible to give it rigidity under aerodynamic loads. It was also determined that a much lighter, more rigid structure could be developed if the torque rod was replaced by a dedicated hinge rod in the leading edge and a actuator rod which is parallel to the hinge rod but set back by 2 inches. The actuator rod has a bend designed into it which cams the feathers to their desired control positions as it rotates in $\mathrm{a}+/-30$ degree arch. The actuator rod passes through a high tolerance slot in each feather and the hinge rod passes through a carbon wrapped thin-wall brass bushing in the leading edge of each feather. The feather and actuator rod details can be seen in Figure 130 and Figure 134, 


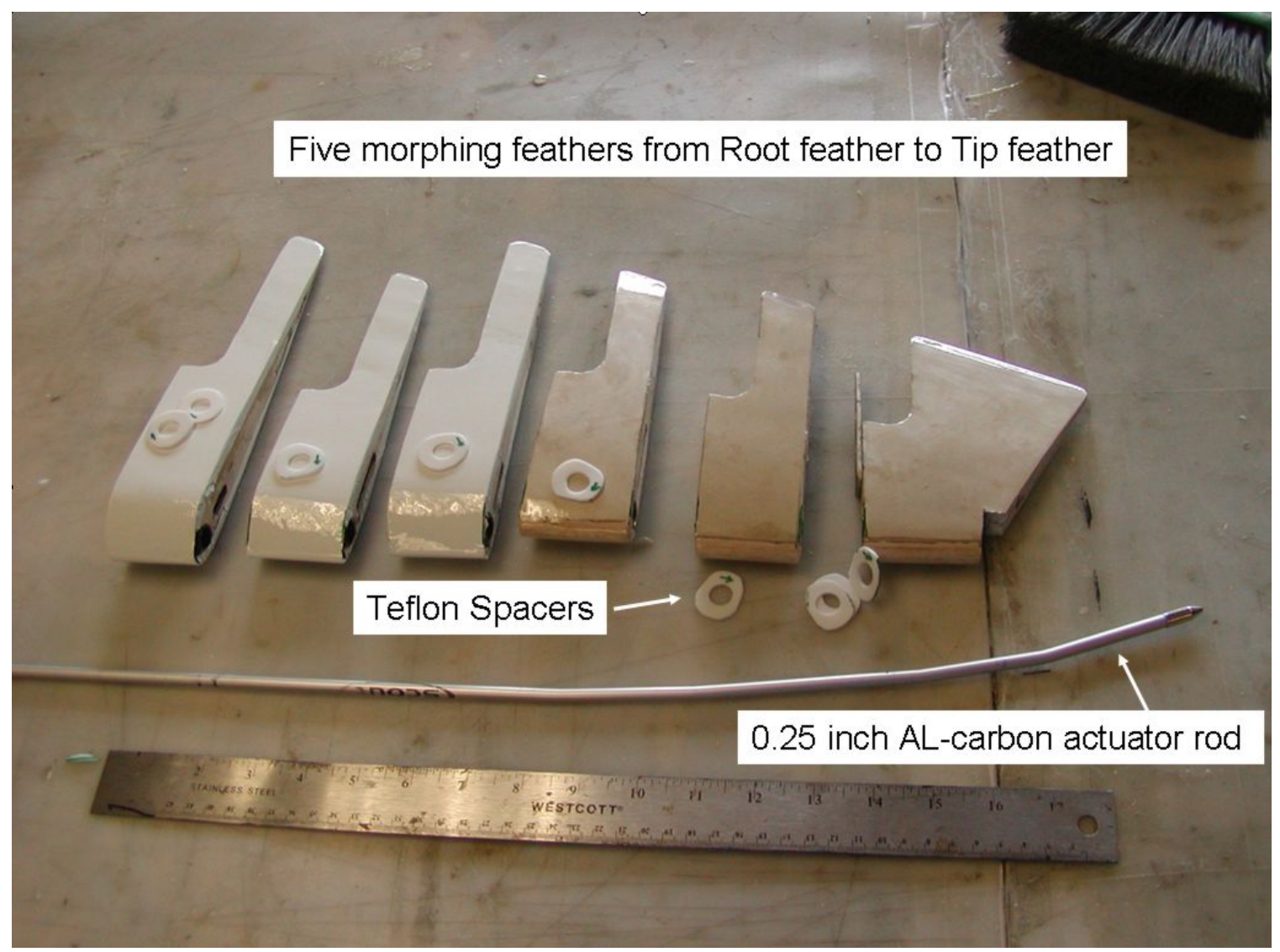

Figure 130.-. Morphing mechanism component details

The actuator rod's maximum possible diameter is $0.25 \mathrm{inch}$ because of its need to pass through the aft portion of the tip feathers. This rod also needs to be light and rigid. After a number of designs, both all metal and all composite, a hybrid design was arrived at. The design began with an Aluminum 6061 T-6 tube with an OD of 0.25 inch and a wall thickness of $0.010 \mathrm{inch}$. This tube could be accurately bend to the desired shape, but in this form lacks the needed strength or rigidity. After the tube was bent it was placed in a jig to hold its shape while $5.7 \mathrm{oz} 3 \mathrm{k}$ carbon roving wetted out with epoxy was compressed and pulled through the tube. The tube was left in the jig for the composite core to cure. 


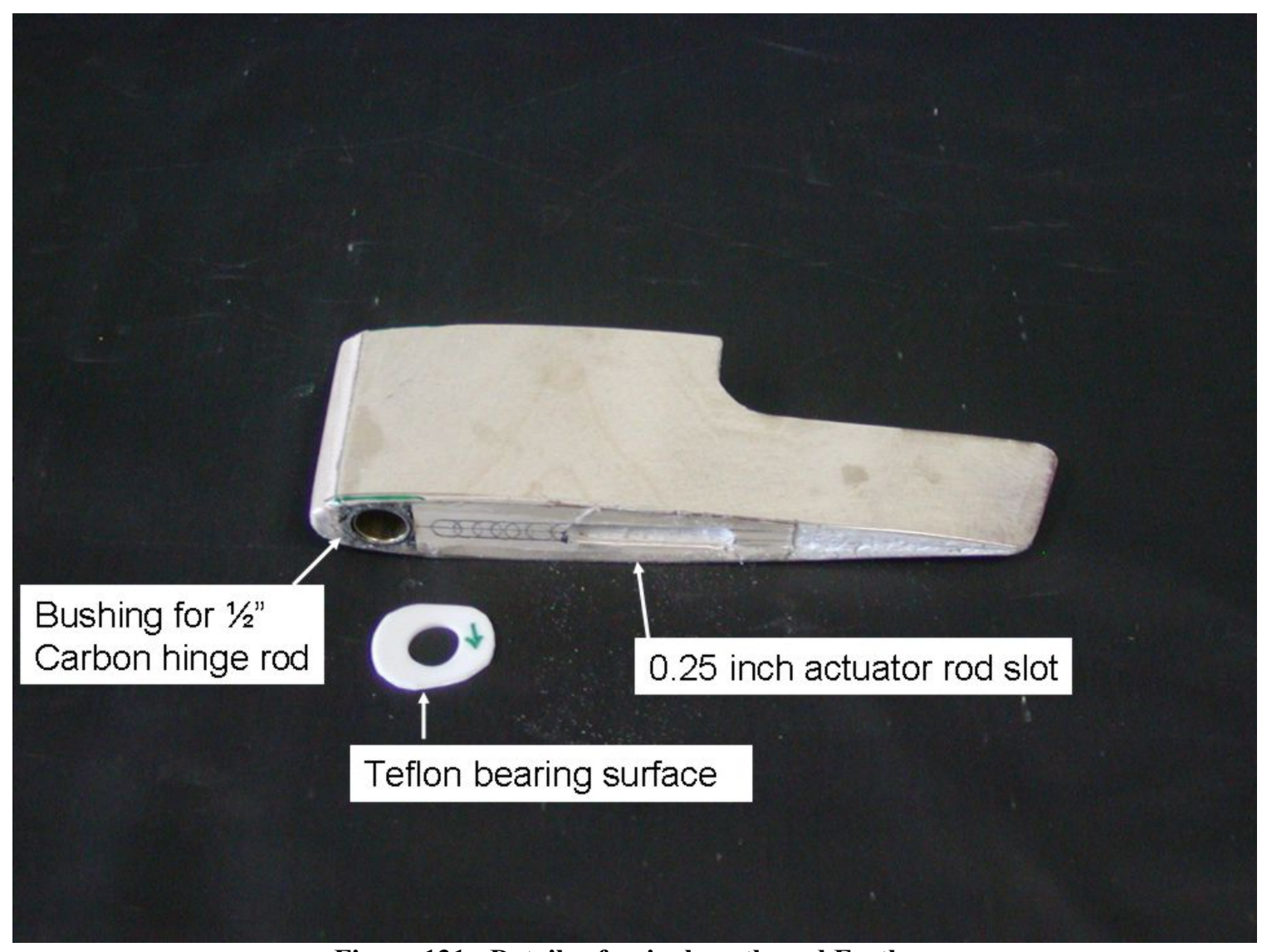

Figure 131.-.Details of a single outboard Feather.

Assembly of the morphing mechanism begins with bonding the carbon hinge rod into the leading edge of the aircraft wing. Once the carbon rod was installed a piece of 0.012 inch Kynar heat shrink tubing was placed over the tube to provide a low friction wear surface and to protect the pultruded carbon rod from fraying. Next the actuator rod was slipped through brass bushings in the wing ribs and a 1" actuator arm was installed to allow the servo to rotate the actuator rod. Then the feathers and Teflon spacers were slipped on from the root to the tip where they are finally held in place by a machine screw and bushing in the hinge rod. The assembled morphing mechanism can be seen in Figure 132 with all assembly complete except for the installation of the tip feather and the retaining screw. This design increases the rigidity over the previous design because the 2" separation of the hinge rod and actuator rod hold the feathers in place. The previous designs depended on the servo and a spring loaded retaining cable in the trailing edge of the wing to hold the structure in place. 


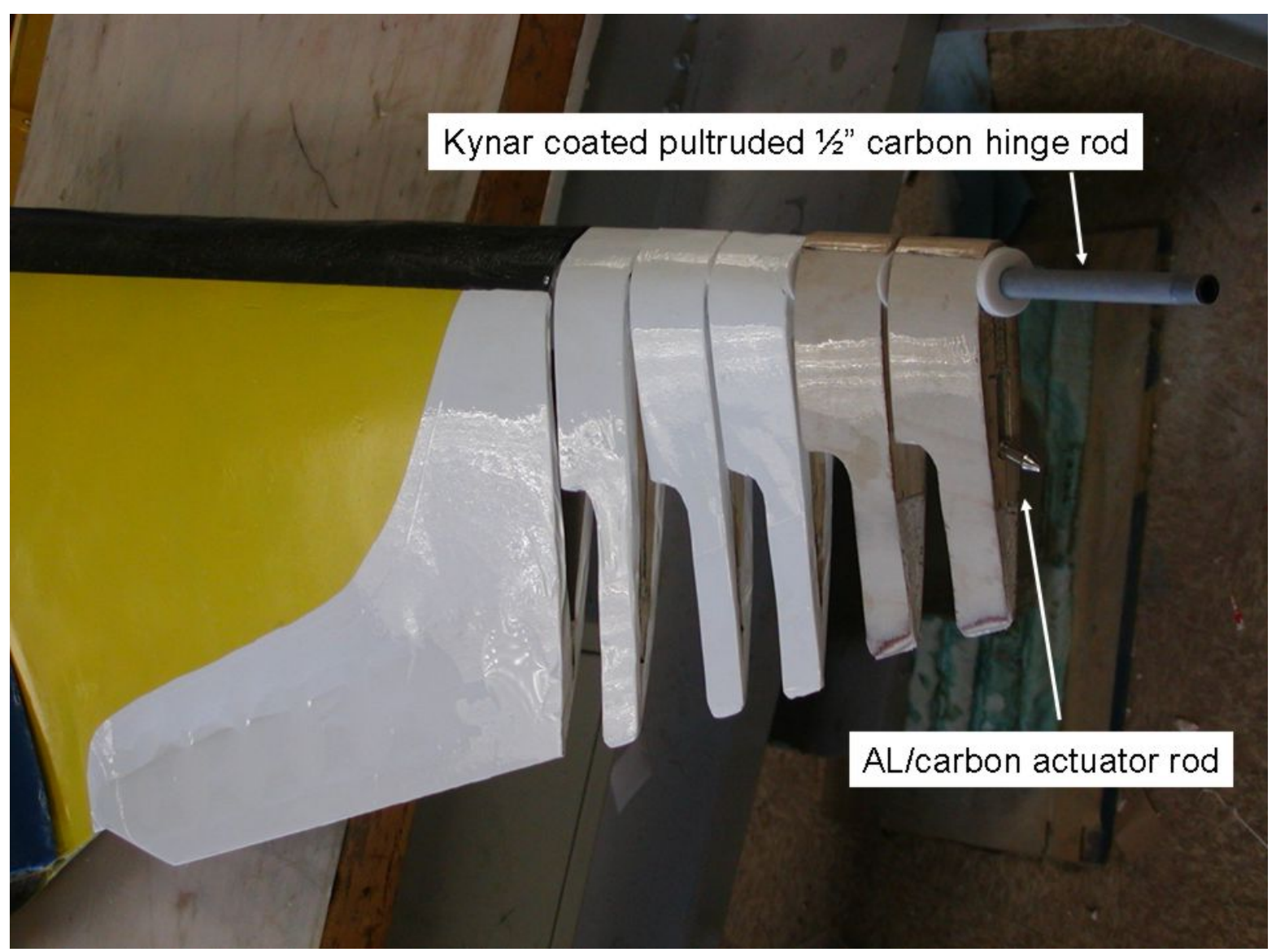

Figure 132 - Morphing mechanisn without tip feather, both the kynar coated carbon hinge rod and the $\mathrm{Al} /$ carbon actuator rods can be seen.

The original skin design was totally unsatisfactory for the flight demonstrator. The original design called for a 0.014 inch neoprene boot to be fitted to each morphing wing section. This skin boot design had three design flaws the first was that each neoprene boot weighed over $1 \mathrm{lb}$ which means that over $20 \%$ of the total aircraft weight would be elastic skins and be behind the aircraft $\mathrm{Cg}$.. The second problem was that the skin needed to be preloaded or stretched when installed in order to resist aerodynamic forces. This preload increased to force that the actuator servo need to overcome which greatly increased the servo current draw and limited the range of motion in the controls. The third problem was that even with a preloaded skin the aircraft would need to maintain a low flight speed to minimize ballooning of the skin. These problems were solved by covering most of the morphing region with a flexible, but non-elastic 0.003 inch polypropylene flim. Only 0.25 inch wide 0.014 thick laytex strips were exposed between each feather. This combination of materials negated the load on the servo the controls when in the neutral control position. The new skin also reduced the skin weight from 2.2 $\mathrm{lb}$ to $0.24 \mathrm{lbs}$. This final skin can be see in Figure 133. The final aircraft weight with data acquisition and propulsion is $10.0 \mathrm{lbs}$, which is $0.3 \mathrm{lbs}$ below the design estimate. 


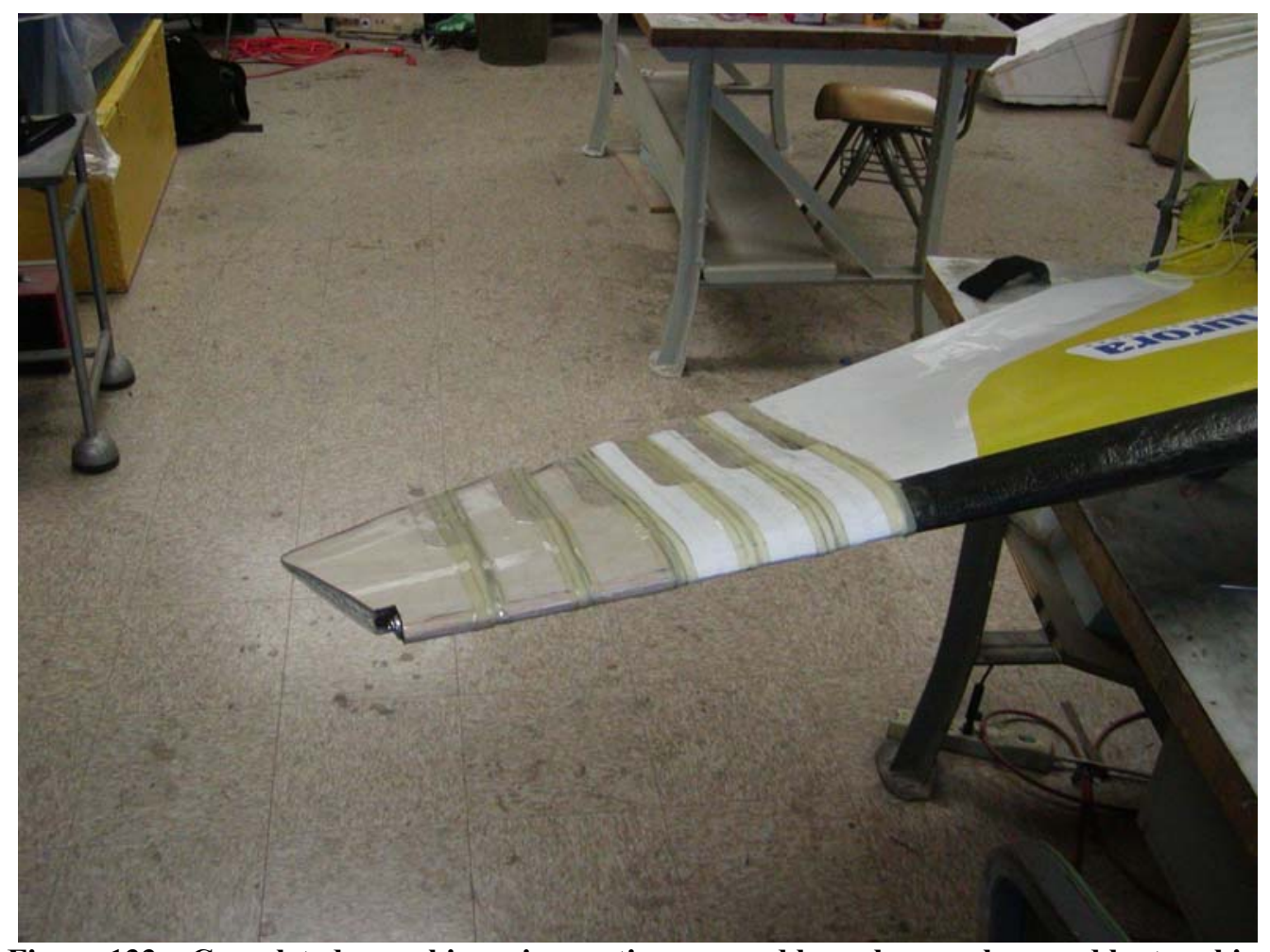

Figure 133 - Completed morphing wing section covered by polypropolene and laytex skin.

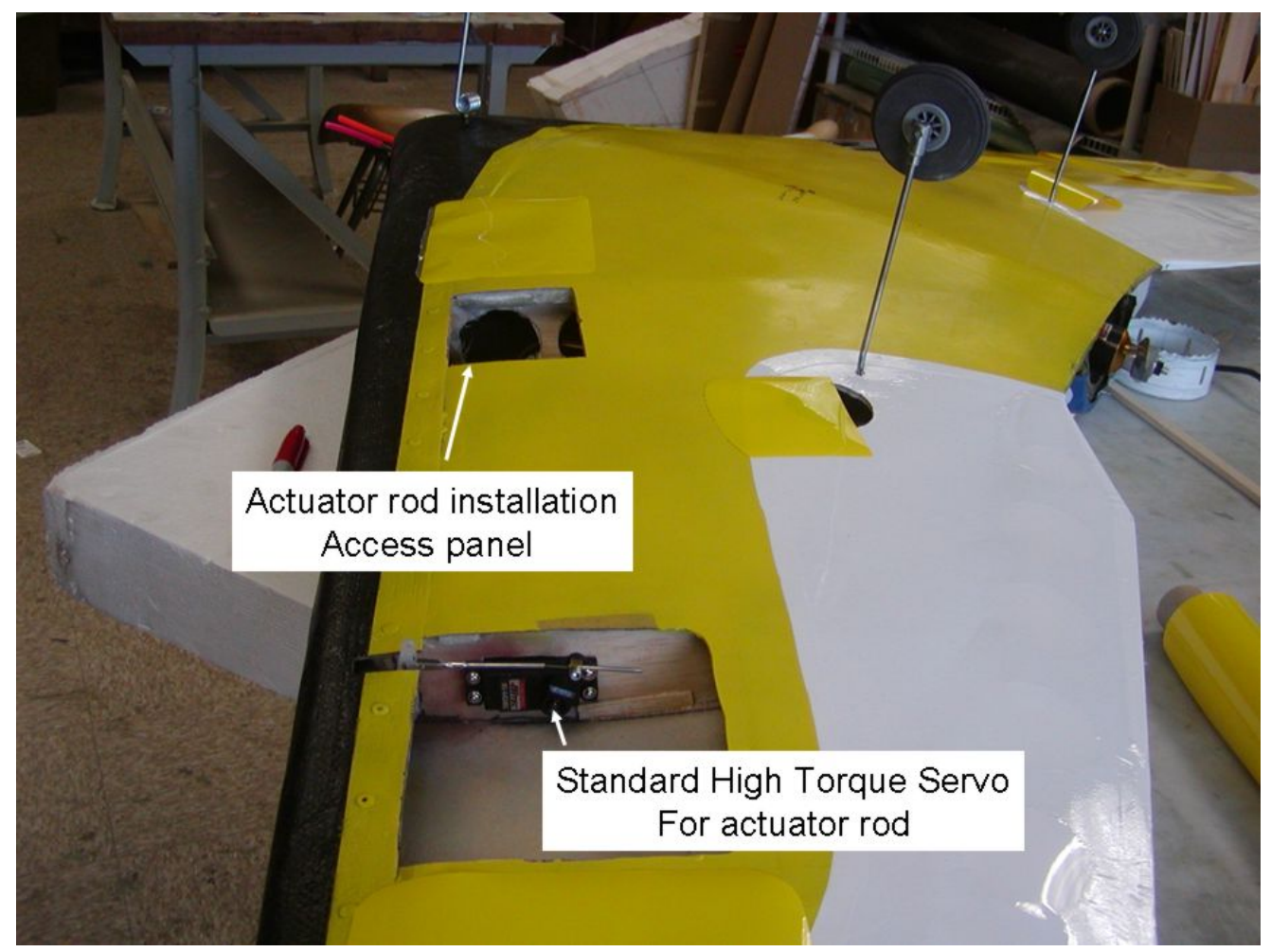

Figure 134 - Servo and actuator arm through accesses panels in bottom od aircraft. 
A Eagletree data flight recorder with a seagull wireless data transmitter which used static pressure / pitot tube/ transducer combination for flight speed and pressure altitude, a magnetic RPM sensor for motor RPMs, thermocouple for temperature and a two axis accelerometer for $g$-forces in the rolling axis. The recorder also recorded the servo positions and control inputs from the radio. Data was recorded at $10 \mathrm{hz}$ and saved on an onboard computer. Velocity, altitude and signal strength were continuously transmitted to a ground station. Figure 135 shows the Habicht II with its main payload bay open. The data acquisition equipment can be accessed in the main payload base. The data recorder utilizes a USB cord to communicate with the Eagletree software loaded on a laptop computer. (Figure 136)

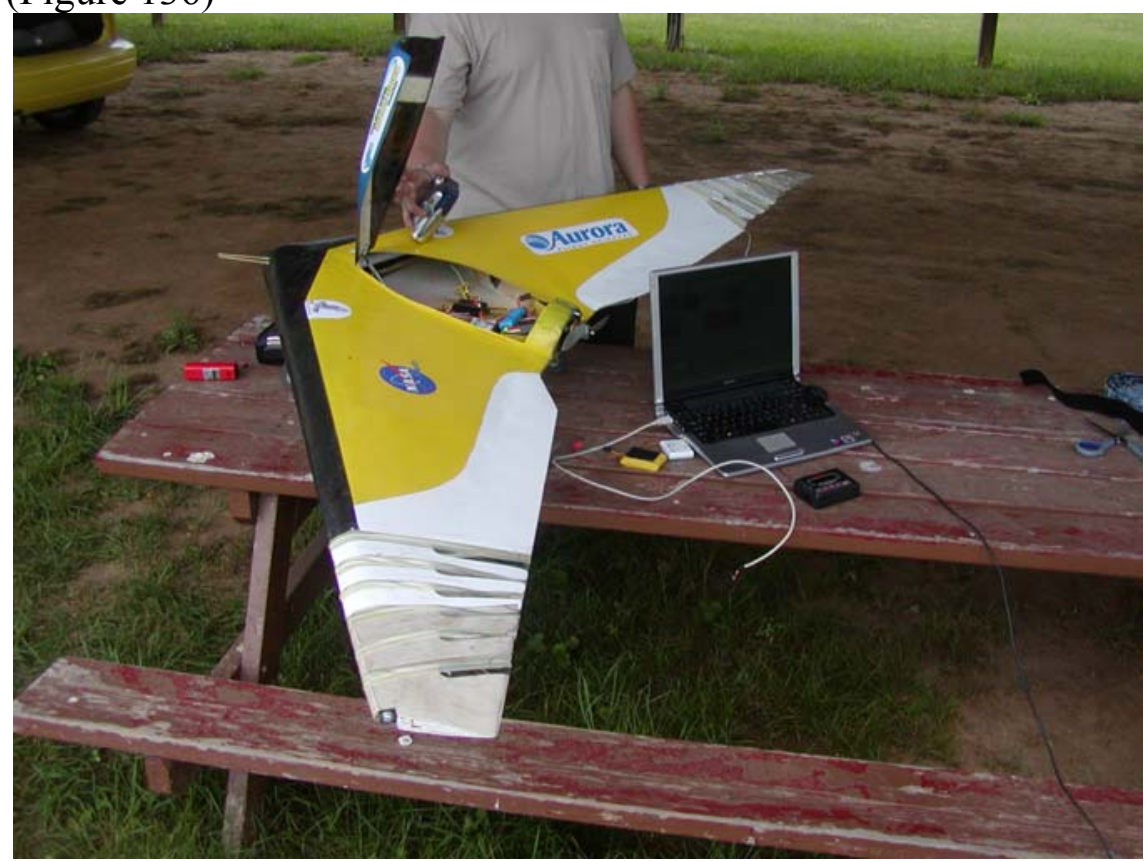

Figure 135 - The Habicht II hooked up for data download.

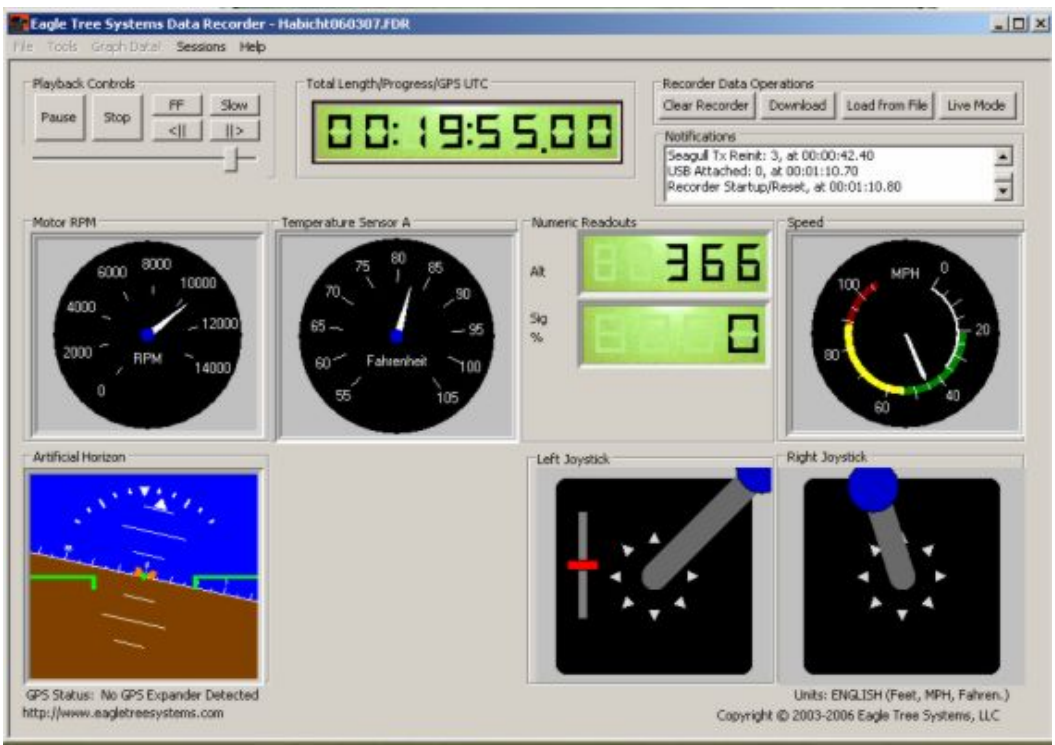

Figure 136 - Eagletree control software with realtime test flight capabilities. 
To minimize the risk of unrecoverable tip stalls 6 degrees of washout was set into the morphing controls. This washout setting corresponded with a desired $\mathrm{Cg}$ location of 60.8 $\%$ aft of the leading edge at the root, which was determined by earlier full scale.glider tests. Figure 137 shows the Habicht II ready for flight with the controls in trim position.

Propulsion was provided by a 680 watt Axi 2408/20 brushless motor turning a 10 x 7 APC glass filled nylon propeller. Thrust test indicated that this motor propeller combination is capable of up to 3.5 pound of trust. Two 14 cell Sanyo NiMh 1900 milliamp hour battery packs in parallel provided enough power for up to 18 minutes of sustained flight.

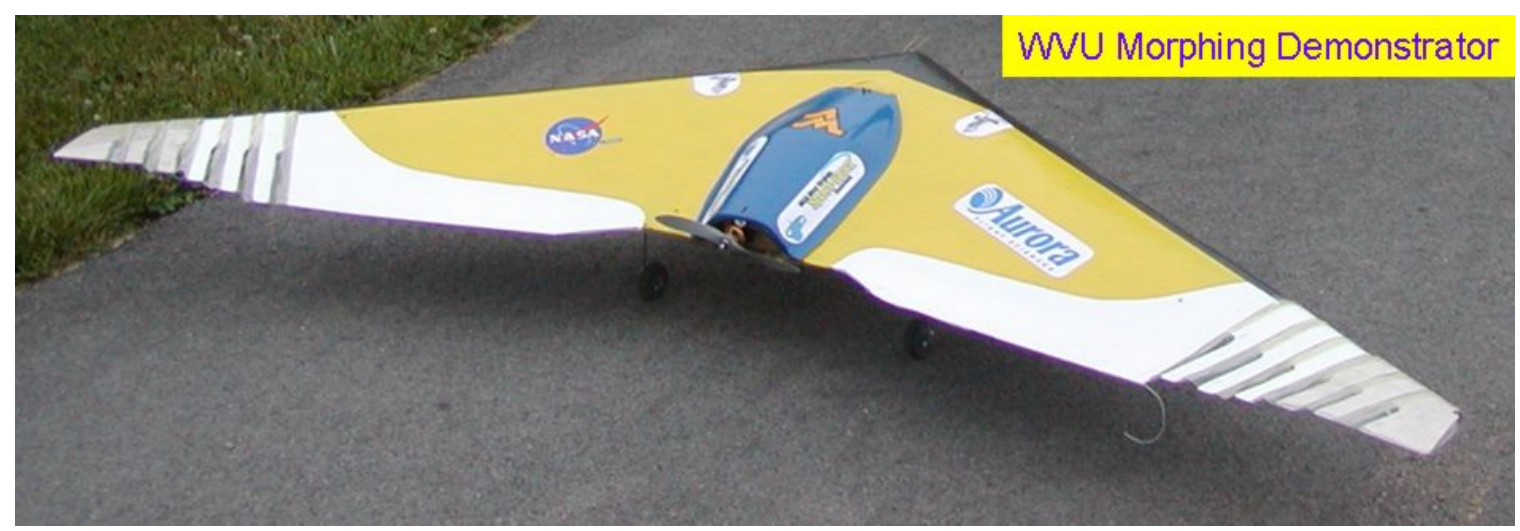

Figure 137 - Habicht II ready for flight with controls in trim position.

A computer control radio capable of mixing was used for control. The morphing surfaces can be used together as an elevator or differentially for an aileron function or in any combination. In their 6 degree washed out position the surface are capable of 12 degrees of twist up or down for control at a throw rate of 60 degrees in 0.24 seconds.

The aircraft was prepared for flight on June $3^{\text {rd }}$, 2007. A UAV pilot, Pete Cooke, was provided by Aurora Flight Sciences. Flight testing took place at WVU's Jaskson Mills Airfield. This airfield is ideal for flight testing because its $50 \mathrm{ft}$ x $2500 \mathrm{ft}$ paved runway surrounded by flat grass land. After taxi tests the flight controls were checked for the last time. Take off was in less than $200 \mathrm{ft}$ at approximately $30 \mathrm{mph}$. The aircraft climbed at $11 \mathrm{ft} / \mathrm{sec}$ and when it was determined that control trim was correct was put into a gentle northward turn. The aircraft put into orbit around the airfield at an altitude of approximately 500 feet. After three orbits, using the data telemetry the aircraft was flow level at a single throttle setting at $29 \mathrm{mph}$. At this velocity the Re is 375000 which matched the wind tunnel data. Most of the tests were conducted between 22 and $40 \mathrm{mph}$. although toward the end of testing the aircraft reached $89 \mathrm{mph}$ in a powered dive. 


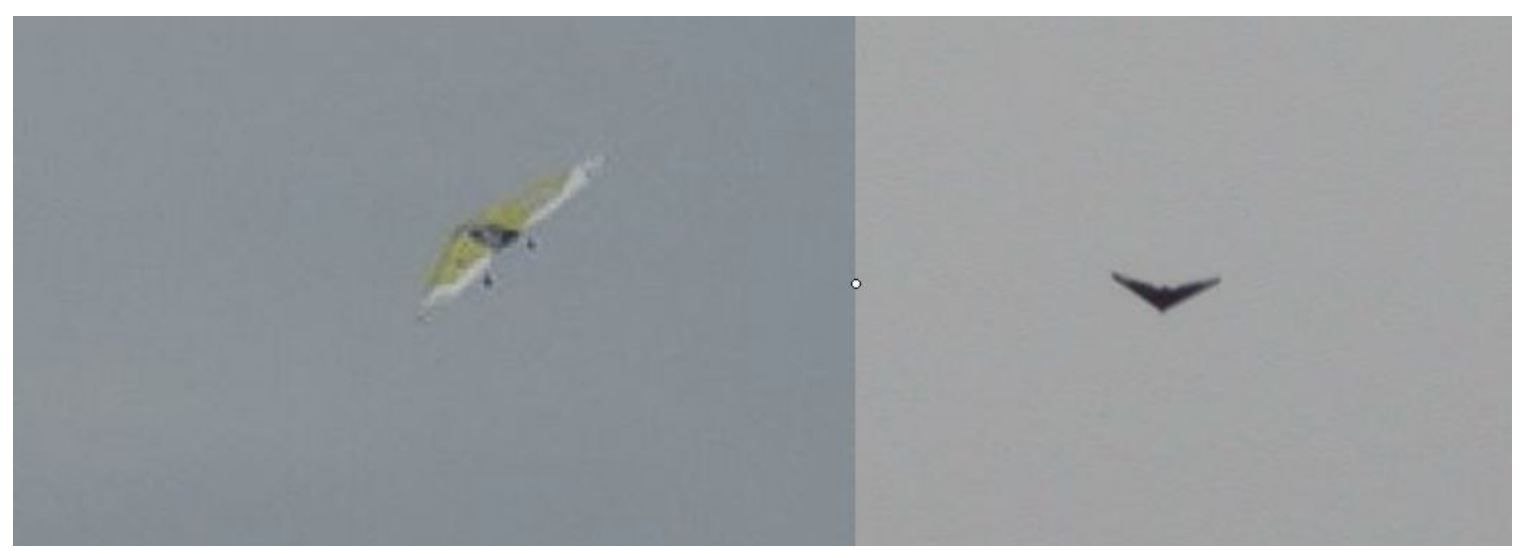

Figure 138- The Habicht $I$ in its first northward turn and on overhead shot during stall tests.

After three orbits in the clockwise direction and a variety of climbs and dives, stall test began at an altitude of $600 \mathrm{ft}$. The stall speed was determined to be approximately 12 mph with gentle nose down stall behavior. After the first set of stall tests the aircraft was allowed to glide from non-accelerating $22 \mathrm{mph}$ level flight. The aircraft maintained an average speed of $22 \mathrm{mph}$ while descending $145 \mathrm{ft}$ over $546 \mathrm{ft}$ in ground distance. This gives the aircraft a glide ratio of 3.8:1, with a 15 degree glide slope. This glide slope can be used an estimate of the lift over drag ratio which agrees well with L/Ds from wind tunnel testing. Then the aircraft was flow level in non-accelerating flight with a constant throttle setting at $36 \mathrm{mph}$. A thrust of $0.956 \mathrm{lbs}$ was determined using the manufactures trust curves for the APC propeller at $36 \mathrm{mph}$ and $7250 \mathrm{RPM}$. Using the equations for Coefficient of lift and drag the $C_{L}$ and $C_{D}$ for the Habicht II were estimated at an assumed 6 degree angle of incidence in level flight and the aircraft weight of 10 pounds.

$$
\begin{gathered}
C_{D}=\frac{F_{\text {drag }}}{1 / 2 \rho_{\text {air }} V^{2} A}, \mathbf{C}_{\mathbf{D}}=\mathbf{0 . 0 4 3} \\
\text { Coefficient of Drag } \\
\mathrm{Fj}=\text { averaged measured loading values (Thrust) }(0.956 \mathrm{Lb}) \\
\mathrm{V} \infty=\text { freestream velocity }(52.8 \mathrm{ft} / \mathrm{sec}) \\
\mathrm{A}=\text { Planform Area }\left(7.15 \mathrm{ft}^{2)}\right. \\
\rho=\text { density }\left(0.0713 \mathrm{lbm} / \mathrm{ft}^{3}\right) \\
C_{L}=\frac{F_{\text {lift }}}{1 / 2 \rho_{\text {air }} V^{2} b c}, \mathbf{C}_{\mathbf{L}}=\mathbf{0 . 4 5 4} \\
\mathrm{Fj}=\text { averaged measured loading values }(\mathrm{Weight})(10 \mathrm{Lb}) \\
\text { V } \infty=\text { freestream velocity }(52.8 \mathrm{ft} / \mathrm{sec}) \\
\mathrm{A}=\text { Planform Area }\left(7.15 \mathrm{ft}^{2}\right) \\
\rho=\text { density }\left(0.0713 \mathrm{lbm} / \mathrm{ft}^{3}\right)
\end{gathered}
$$

To determine roll rate, the aircraft was rolled 4 consecutive rolls giving it an average 378 degree/sec roll rate. Then the second set of stall tests was initiated. The pilot attempted to 
get the aircraft to go into a tip stall but was unable to. The elevon equipped demonstrator could be put into a tip stall easily. The pilot noted that roll and yaw behavior was very similar to the elevon equipped aircraft, but pitch was approximately $1 / 3$ less responsive. Finally the aircraft was put into a powered dive to explore the morphing mechanisms behavior at higher speeds. A velocity of $89 \mathrm{mph}$ was reached with out any unusual behavior being observed. This concluded a15 minute flight testing program. Figure 138 shows the Habicht in flight. Figure 139 is an example of some of the data collected.

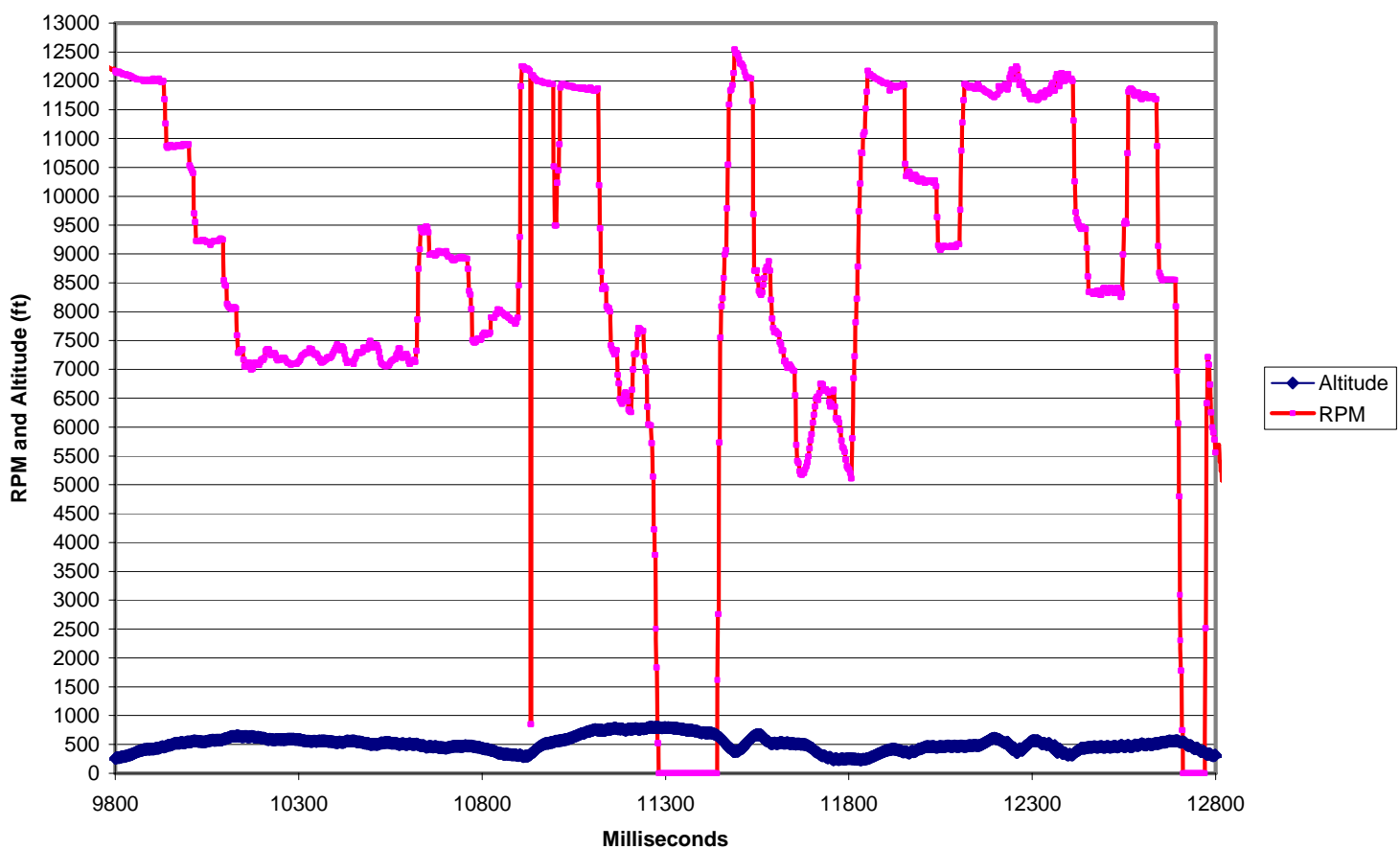

Figure 139 - Example Altitude and propeller RPM data collected from flight testing.

\section{Error analysis}

Error and Uncertainty can be defined in a variety of ways therefore for the purpose of clairity the following definitions will be used in this research.

Precision Errors. Precision errors, also called random errors due to their nature, can have various sources. They are often associated with the "least count" of the scale on an analog instrument. For example, if a manometer has graduations at intervals of $1 \mathrm{~mm}$ (a least count of $1 \mathrm{~mm}$ ), we can expect precision errors of about $1 \mathrm{~mm}$ in readings of the manometer due to the way we eye the scale. Precision errors also arise in analog to digital conversions of signals. For example, an 8-bit A/D converter can record 256 voltage levels: if it is to have a range of $10 \mathrm{~V}$, precision errors of about $10 / 256=0.04 \mathrm{~V}$ can be expected. 
Bias Errors. Bias errors, also called systematic errors, have many sources. The source most often mentioned in texts is a calibration error in a measuring instrument. Calibration errors may be a zero-offset error that causes a constant absolute error in all readings, or a scale error in the slope of output versus input that causes a constant percentage error in all readings. Scale errors are also called sensitivity or span errors. Some instruments have a bias error associated with hysteresis, that is, the output differs depending on whether the input is increasing or decreasing.

Accuracy. Often instrument manufacturers quote an "accuracy" for their instrument, for example $\pm 0.5 \%$. Of course, a maximum error of $\pm 0.5 \%$ is implied (the instrument is 99.5\% accurate) (Mills, 2004)

The major sources of error and uncertainty for this research are the following, mercury thermometer, mercury manometer, pressure tranducer, and electromagnetic noise . These errors and uncertainty sources will be evaluated and discussed in this section as well as the propagation of systematic errors

The ISO standard for error analysis has been used as a guideline for this error analysis. In summary this ISO standard approach is to first estimate the elemental errors and their contribution to the measured variables. A recommended procedure for estimating variable systematic and random limits follows:

1) For an experiment, determine the data reduction equation (DRE) and identify the important variables. Determine how well the final result needs to be known. This will give a guideline how much uncertainty in each variable can be tolerated.

2) After identifying the important variables, estimate the range of anticipated test conditions. Next, assume all uncertainties are random at first (precision).

Investigate the sensitivity of the uncertainty in the result to the uncertainties in the variables. Focus on the variables whose uncertainties will affect the results the most.

3) Concentrate on obtaining detailed estimates of the uncertainties in the most important variables.

4) Perform a detailed uncertainty analysis considering systematic (bias) and precision (random) uncertainties. If precise bias and precision limits are unknown, make a reasonable estimate based on the most limiting measurement.

a. Usually variables have elemental uncertainty sources, which contributes to its uncertainty. Determine their relative significance first and use an order of magnitude estimate to eliminate insignificant elemental sources. "Rule of thumb for a given variable - those uncertainty sources that are smaller than $1 / 4$ or $1 / 5$ of the largest sources can usually be considered negligible.”. 
b. Often it is not cost effective or necessary to try to estimate precision limits at the elemental level. These individual elemental uncertainties are often insignificant to the desired result. A more effective approach is to estimate the precision limits of the measured variables at the variable level. (Yen, 2000)

To calculate the force and moment coefficients from wind tunnel data, CL, CD and CMP, CMY, CMR, the following equations are used. These equations represent the five Data Reduction Equations. used for uncertainty analysis, where $\mathrm{Fj}, \mathrm{Mj}, \mathrm{V}, \mathrm{b}, \mathrm{c}$, and $\rho$ are the important variables.

$$
C_{L}=\frac{F_{\text {lift }}}{1 / 2 \rho_{\text {air }} V^{2} b c}
$$

Coefficient of Lift

$$
C_{D}=\frac{F_{\text {drag }}}{1 / 2 \rho_{\text {air }} V^{2} b c}
$$

Coefficient of Drag

$$
C_{M P}=\frac{M_{\text {pitch }}}{1 / 2 \rho_{\text {air }} V^{2} b c c}
$$

Pitching Moment Coefficient

$$
C_{M Y}=\frac{M_{\text {yawt }}}{1 / 2 \rho_{\text {air }} V^{2} b c b}
$$

Yawing Moment Coefficient

$$
C_{\text {MR }}=\frac{M_{\text {roll }}}{1 / 2 \rho_{\text {air }} V^{2} b c b}
$$

Rolling Moment Coefficient

$$
\begin{gathered}
\mathrm{Fj}=\text { averaged measured loading values } \\
\mathrm{Mj}=\text { averaged measured moment values } \\
\mathrm{V} \infty=\text { freestream velocity } \\
\mathrm{k}=\text { correction term } \\
\mathrm{b}=\text { airfoil span } \\
\mathrm{c}=\text { airfoil chord } \\
\rho=\text { density }
\end{gathered}
$$

Elemental Error Sources

To determine the precision and bias associated with the experimental results an uncertainty analysis is performed on the raw data at the elemental level. Contributing factors to uncertainty include resolution of measurement systems and other test condition variability's.

Error order of magnitude analysis on the data reduction equations indicated the most significant variable is the air velocity due to its quadratic nature. Velocity is then followed by the load and moment values. Air Velocity $(\mathrm{V} \infty)$ is dependant on air density $\left(\rho_{\text {air }}\right)$, air temperature $\left(\mathrm{T}_{\text {atm }}\right)$, total pressure, which equals Atmosperic Pressure $(\mathrm{Patm})+$ Tunnel Dynamic Pressure (q).The error for each of these components a has been calculated for the $\mathrm{V} \infty$ variable and then have been combine with a sample data set which will identify the worst case error scenario for the calculation of $\mathrm{V} \infty$. These calculations can be seen below in Table 10 through Table 16. 
Table 10 - Velocity calculation error analysis.

\begin{tabular}{lll}
\hline \multicolumn{4}{l}{ Velocity Calculation Error Analysis } \\
Temperature Measurement \\
Instrument: & Mercury Thermometer \\
Range: & $.-30-140$ & degrees F \\
Accuracy: &.$+/-0.5$ & degrees F
\end{tabular}

Atmospheric Pressure Measurement

Insturment : Mercury Barometer
Range :
28-32
in. $\mathrm{Hg}$
Accuracy:
$.+1-0.05$
in. $\mathrm{Hg}$
$\mathrm{P}_{\text {atm }+/-}(\mathrm{psi})$
0.02
$\mathrm{P}_{\text {atm }+/-}(\mathrm{psf})$
3.54

Wing Tunnel Dynamic Differential Pressure Measurement

Instrument : $\quad$ Dwyer Series 621 Model N18Q

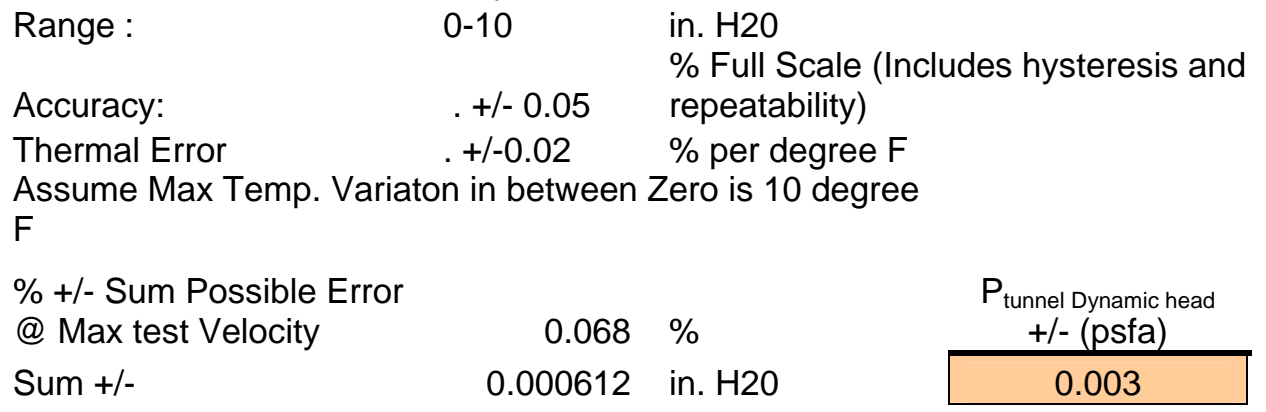

Air Density Error

Analysis

Sum or Error +/- (psf)

Pressure total $=$ Pressure

Tunnel Dynamic +

Atmospheric Pressure

Temperature

3.54 (psf)

0.5 degree $F$

Sample Plus

Max Error in all

\begin{tabular}{|c|c|c|c|c|}
\hline & $\begin{array}{l}\text { Sample } \\
(\mathrm{lbm} / \mathrm{ft} 3)\end{array}$ & $\begin{array}{l}\text { Components } \\
(\mathrm{lbm} / \mathrm{ft} 3)\end{array}$ & $\begin{array}{l}\text { Error Magnitude } \\
(\mathrm{lbm} / \mathrm{ft} 3)\end{array}$ & $\%$ Error +/- \\
\hline \multirow[t]{2}{*}{ Air Density Error } & 0.0713 & 0.0713 & 0.0001 & 0.0798 \\
\hline & $\begin{array}{l}\text { Sample } \\
\text { (ft/sec) }\end{array}$ & $\begin{array}{l}\text { Sample Plus } \\
\text { Max Error in all } \\
\text { Components } \\
\text { (ft/sec) }\end{array}$ & $\begin{array}{l}\text { Error Magnitude } \\
\text { (ft/sec) }\end{array}$ & $\%$ Error $+/-$ \\
\hline Velocity Infinity Error & 10.41 & 10.54 & 0.13 & 1.28 \\
\hline
\end{tabular}


Load and moment values were measured using the NASA \#626 six component balance, so the major sources of error from the balance and data acquisition system combination have been identified. The first source of error identified for the balance is the hysteresus found in each strain gage bridge combination. The hysteresus effects were identified in load tests and calibrations that were conducted on each channel of the balance and are included in Appendix A. Electromagnetic noise has been a major problem during this project and has been minimized in every way possible, but still must be accounted for. During load tests 300 data samples were recorded while a constant maximum load was applied to the channel of interest. The maximum variation during these tests was recorded and used for a worst case senerio for noise encounters during testing. Noise and hysteresus are the significant sources of error for the balance system. Table 11 summarizes the error calculation for the balance system.

Table 11 - Force balance system error analysis.

\begin{tabular}{|c|c|c|c|c|c|}
\hline & $\begin{array}{c}\text { Max Observed } \\
\text { Hysteresus for each } \\
\text { channel }+/-\end{array}$ & Units & $\begin{array}{c}\text { Manufacturer } \\
\text { Accuracy } \% \text { of } \\
\text { Full Scale (95\% } \\
\text { confidence) }\end{array}$ & $\begin{array}{c}\text { Max Variation } \\
\text { due to noise in } \\
300 \text { samples @ } \\
\text { Max observed } \\
\text { channel } \\
\text { reading } \\
\end{array}$ & Units \\
\hline Lift & 0.04 & $\mathrm{lb}$ & 0.0026 & 0.08 & $\mathrm{lb}$ \\
\hline Drag & 0.03 & $\mathrm{lb}$ & 0.0010 & 0.01 & $\mathrm{lb}$ \\
\hline Pitch & 0.13 & in-lb & 0.0020 & 0.115 & in-lb \\
\hline Yaw & 0.025 & in-lb & 0.0014 & 0.056 & in-lb \\
\hline Roll & 0.01 & in-lb & 0.0017 & 0.177 & in-lb \\
\hline
\end{tabular}

\begin{tabular}{c|c|c|c|c|}
$\begin{array}{c}\text { Maximum } \\
\text { magnitude } \\
\text { observed channel } \\
\text { reading }\end{array}$ & Units & $\begin{array}{c}\text { \% Max observed } \\
\text { variation due to } \\
\text { EM noise }\end{array}$ & $\begin{array}{c}\text { \% Max } \\
\text { observed } \\
\text { variation due } \\
\text { to Hysteresus }\end{array}$ & $\begin{array}{c}\%+/ \text { - Sum } \\
\text { Max } \\
\text { Observed } \\
\text { Error }\end{array}$ \\
\hline 3.37 & lb & 0.02 & 0.01 & 3.69 \\
\hline 3.37 & lb & 0.00 & 0.01 & 1.43 \\
\hline 4.09 & in-lb & 0.03 & 0.03 & 6.20 \\
\hline 2.39 & in-lb & 0.02 & 0.01 & 3.55 \\
\hline 4.09 & in-lb & 0.04 & 0.00 & 4.75 \\
\hline
\end{tabular}

Another, although small, source of error is the geometric deviation inherit in the wind tunnel models due to the limitations of the fabrication techniques used in their construction. Model measurements compared to computer model indicate that there is a tolerance of $+/-0.030$ inch in wing cord and $+/-0.040$ inch in wing span. These combine to give a error of $+/-0.009$ square feet in the area calculations. Geometric error due to aeroelasticity is factor which has not been accounted for in this study because of the inability to measure the model while the aerodynamic loads are applied. 
The calculation of error for the aerodynamic force and moment coefficients was conducted by combining the $\mathrm{V} \infty$ and the force and moment load errors to give a worst case error situation then percent error was calculated for each coefficient. These calculations are included in the following tables.

Table 12- Coefficient of lift error analysis

\begin{tabular}{|c|c|c|c|c|}
\hline & $\begin{array}{l}\text { Sample } \\
\text { Using Max } \\
\text { Value }\end{array}$ & $\begin{array}{l}\text { Sample Plus } \\
\text { Max Error in all } \\
\text { Components }\end{array}$ & Error Magnitude & $\%$ Error $+/-$ \\
\hline Coefficient of Lift Error & 0.812 & 0.858 & 0.046 & 5.660 \\
\hline
\end{tabular}

Table 13 - Coefficient of drag error analysis

\begin{tabular}{|c|c|c|c|c|}
\hline & $\begin{array}{l}\text { Sample } \\
\text { Using Max } \\
\text { Value }\end{array}$ & $\begin{array}{l}\text { Sample Plus } \\
\text { Max Error in all } \\
\text { Components }\end{array}$ & Error Magnitude & $\%$ Error $+/-$ \\
\hline $\begin{array}{c}\text { Coefficient of Drag } \\
\text { Error }\end{array}$ & 0.812 & 0.841 & 0.029 & 3.541 \\
\hline
\end{tabular}

Table 14- Pitching moment coefficient error analysis

\begin{tabular}{|ccccc|}
\hline & $\begin{array}{c}\text { Sample } \\
\text { Using Max } \\
\text { Value }\end{array}$ & $\begin{array}{c}\text { Sample Plus Max } \\
\text { Error in all } \\
\text { Components }\end{array}$ & Error Magnitude & \% Error +/- \\
& & & & \\
$\begin{array}{c}\text { Coefficient of } \\
\begin{array}{c}\text { Pitching Moment } \\
\text { Error }\end{array}\end{array}$ & 0.720 & 0.747 & 0.027 & 3.818 \\
\hline
\end{tabular}

Table 15- Yawing moment coefficient error analysis

\begin{tabular}{|c|c|c|c|c|}
\hline & $\begin{array}{l}\text { Sample } \\
\text { Using Max } \\
\text { Value }\end{array}$ & $\begin{array}{c}\text { Sample Plus Max } \\
\text { Error in all } \\
\text { Components }\end{array}$ & Error Magnitude & $\%$ Error +/- \\
\hline $\begin{array}{l}\text { Coefficient of } \\
\text { Yawing Moment } \\
\text { Error }\end{array}$ & 1.152 & 1.196 & 0.044 & 3.861 \\
\hline
\end{tabular}

Table 16- Rolling moment coefficient error analysis

\begin{tabular}{|c|c|c|c|c|}
\hline & $\begin{array}{l}\text { Sample } \\
\text { Using Max } \\
\text { Value }\end{array}$ & $\begin{array}{c}\text { Sample Plus Max } \\
\text { Error in all } \\
\text { Components }\end{array}$ & Error Magnitude & $\%$ Error +/- \\
\hline $\begin{array}{l}\text { Coefficient of } \\
\text { Rolling Moment } \\
\text { Error }\end{array}$ & 1.971 & 2.072 & 0.102 & 5.166 \\
\hline
\end{tabular}


The percent errors calculated in Table 12 through Table 16 are indicated on all relevant data graphs with error bars. Both angle of attack and control deflection were measured within 0.25 degrees and this uncertainty is presented as "Y error bars" on all relevant data graphs. Data collected from the flight demonstrator are presented as approximated values due to a wide variety of unaccounted for variables; therefore error analysis of this data is not dealt with in this study.

The differences in the flow conditions when comparing clean wind tunnel conditions and the wind tunnel with a model, due to blockage are usually taken into account by introducing corrections, e.g. when calculating lift or drag coefficients. All the wind tunnel models used in this research had wind tunnel blockage factors less than $2 \%$. With the blocked area being smaller than $10 \%$ of the wind tunnel cross section, it is assumed that the error due to blockage can be neglected.

\section{Discussion of Results}

An adaptive washout morphing mechanism with comparable control characteristics to a similar sized and designed Horten type sweptwing tailless aircraft has been developed. Wind tunnel and CFD testing indicates that lift over drag ratios that are on the order of $40 \%$ better are possible with morphing wing when compared to the elevon equipped wind tunnel model used in this research. The data collected so far is in a critical Reynolds number range as can be seen in Figure 140. It is important to note that due the taper in the models used in this research, Reynolds number varies from 67,340 to 505,048 , from tip to the root. The mean aerodynamic cord was used for all Reynolds numbers presented. Figure 140 shows the drag coefficient; lift Coefficient and L/D as a function of Reynolds number for the elevon equipped (Horten Type Wing). When Figure 140 is compared with Figure 141 which is an adaptation of Prandl's Experimental Drag Coefficients for AxiSymetric Bodies it appears that the test velocities are at a Reynolds number where $C_{D}$ changes rapidly. 


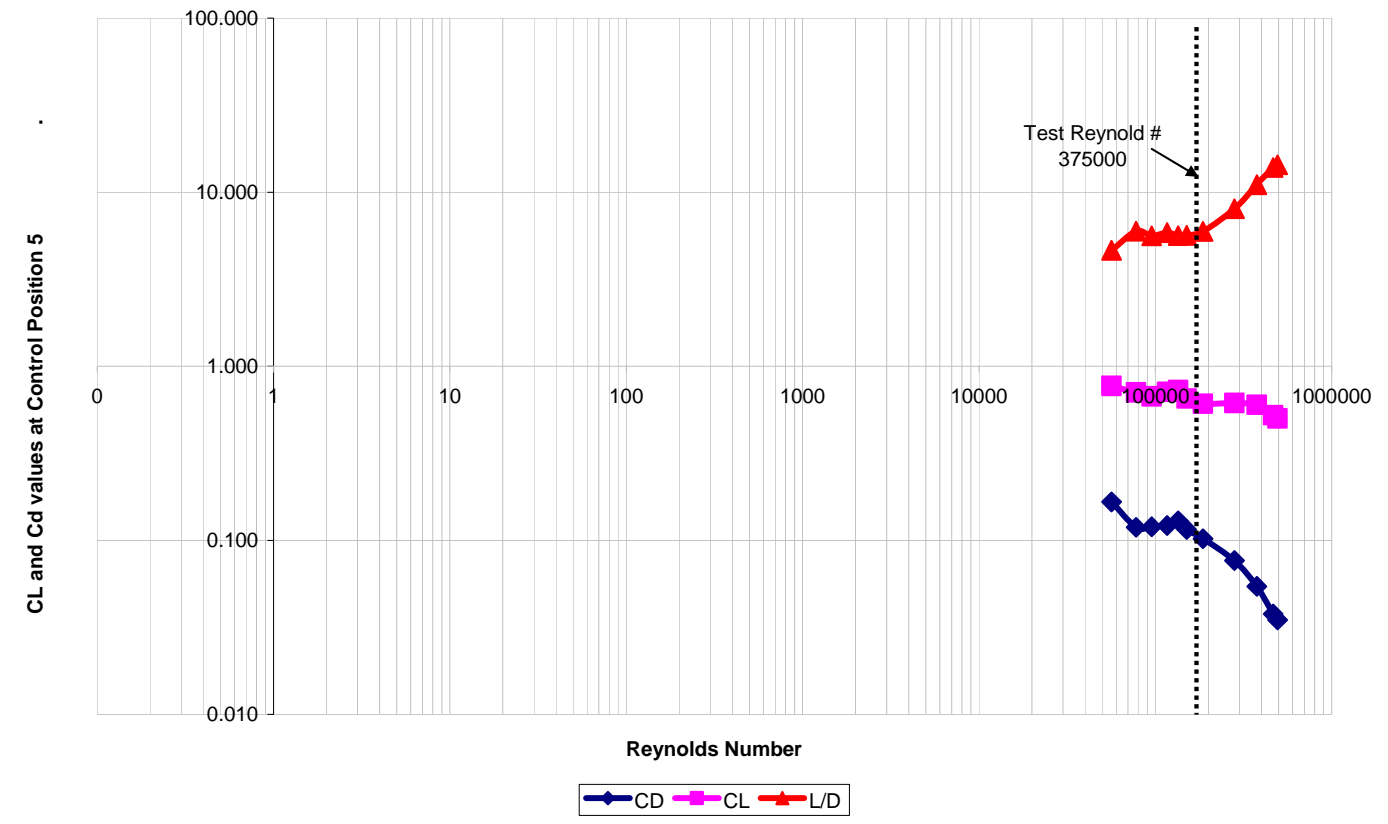

Figure 140 - CD, CL and L/D at increasing Re for the Elevon equipped wing at 7 degree AOA and in control position 5.

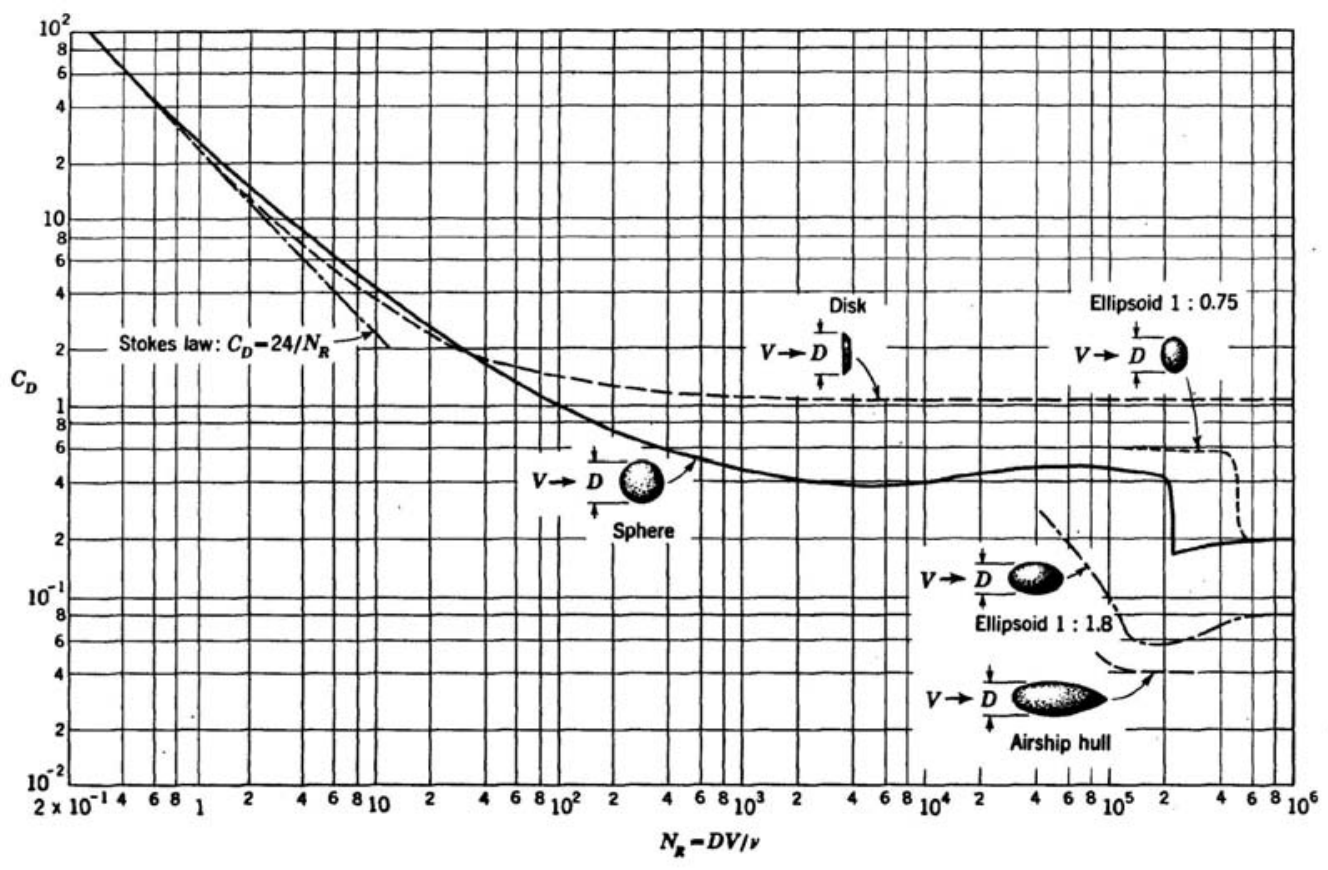

Figure 141 - Experimental Drag Coefficient for Axi-Symmetric bodies. (Adapted from L. Prandl, "Ergebnisse der Aerodynamischen Versuganstalt zu Gottingen," p29, R. Oldenbourg, Munich and Berlin, 1923; and F. Eisner, "Das Wiederstandproblem", Proc. $3^{\text {rd }}$ Internatn. Congr. Appl. Mech, p 32, 1930.

Data on the Horten type wing began to show the unique aerodynamics designed into this type of wing to mitigate adverse yaw. At the control position with the highest $\mathrm{C}_{\mathrm{L}}$, control 
position 5 , the $C_{D}$ is unexpectedly the lowest and the Yaw moment is negative. This behavior also has a Reynolds number dependence and is only evident at velocities greater then $15 \mathrm{~m} / \mathrm{s}$ or a Reynolds number of around 300,000.

Flow visualization techniques using polyester tufts helped define some of the tip vortex behavior and when combined with CFD results, have shed some light on how the Horten type wing and the morphing wing which mimics it, are able to generate pressure fields were the highest $C_{L}$ and $C_{D}$ correspond at the same control position. The tufts indicated when the tip vortex died out and when it reversed with varying control positions. It could be seen that the local AOA at the wing tip was not as large as seen at the Root of the wing and that the Lift vectors at the tip may rotate forward in certain control position in order to reduce drag and reverse yaw locally. This behavior is shown in Figure 142 and Figure 143, which come from RC Soaring Digest which explains this phenomena for a twisted swept wing tailless aircraft.
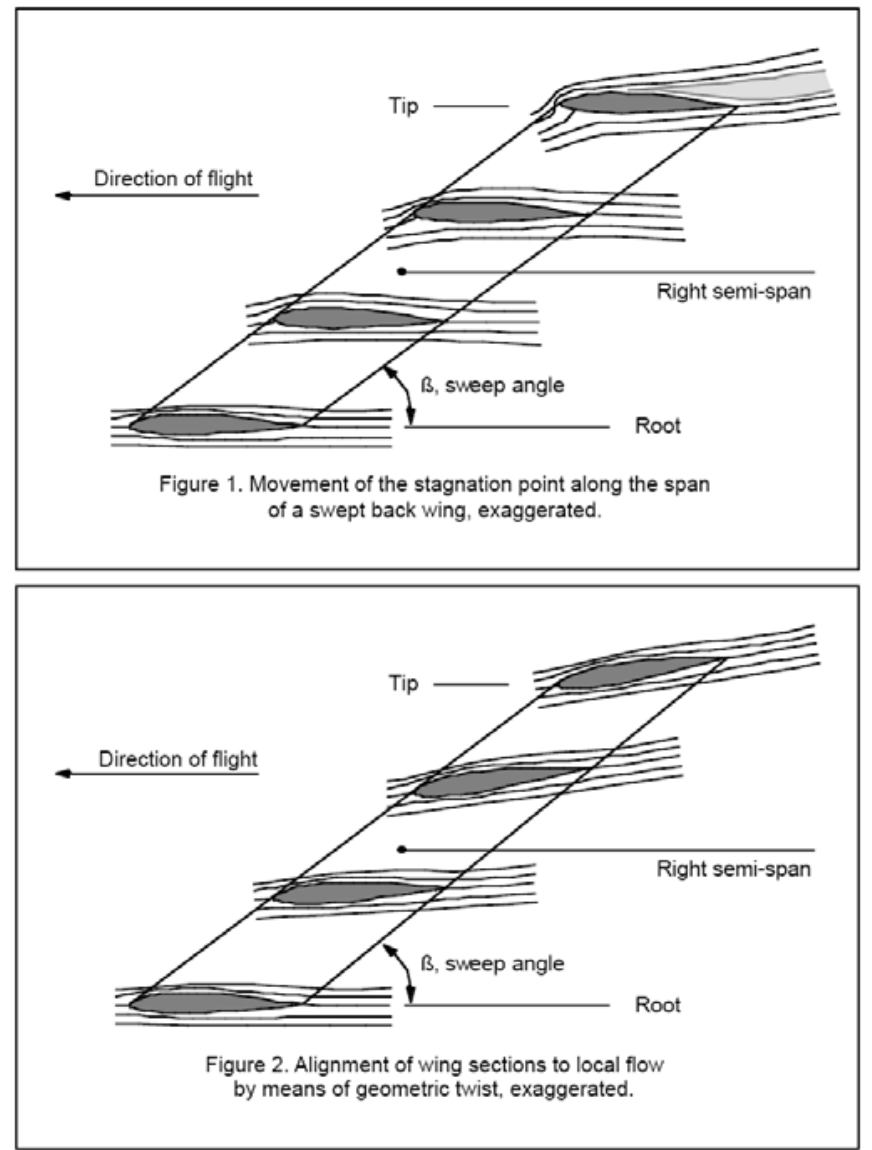

Figure 142 - Wing sweep and twist and their effect on local AOA (Kuhlman, 2002) 

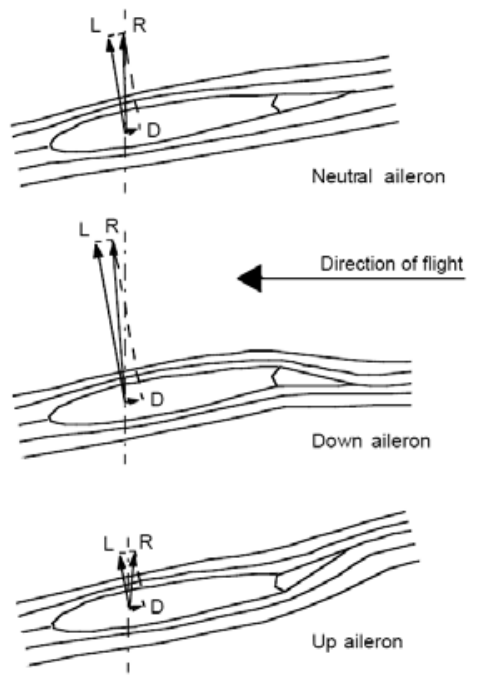

Figure 7. Generalized representation of the direction and strength of forces when the outboard aileron of a twisted swept wing is deflected.

Figure 143 - Outboard aileron and the rotation of the lift vector on a twisted swept wing tailless aircraft - (Kuhlman, 2002)

Wind tunnel, flight demonstrator and CFD data support each other very well in this study Figure 144 through Figure 147 summarize coefficient of lift and drag data from the three sources.

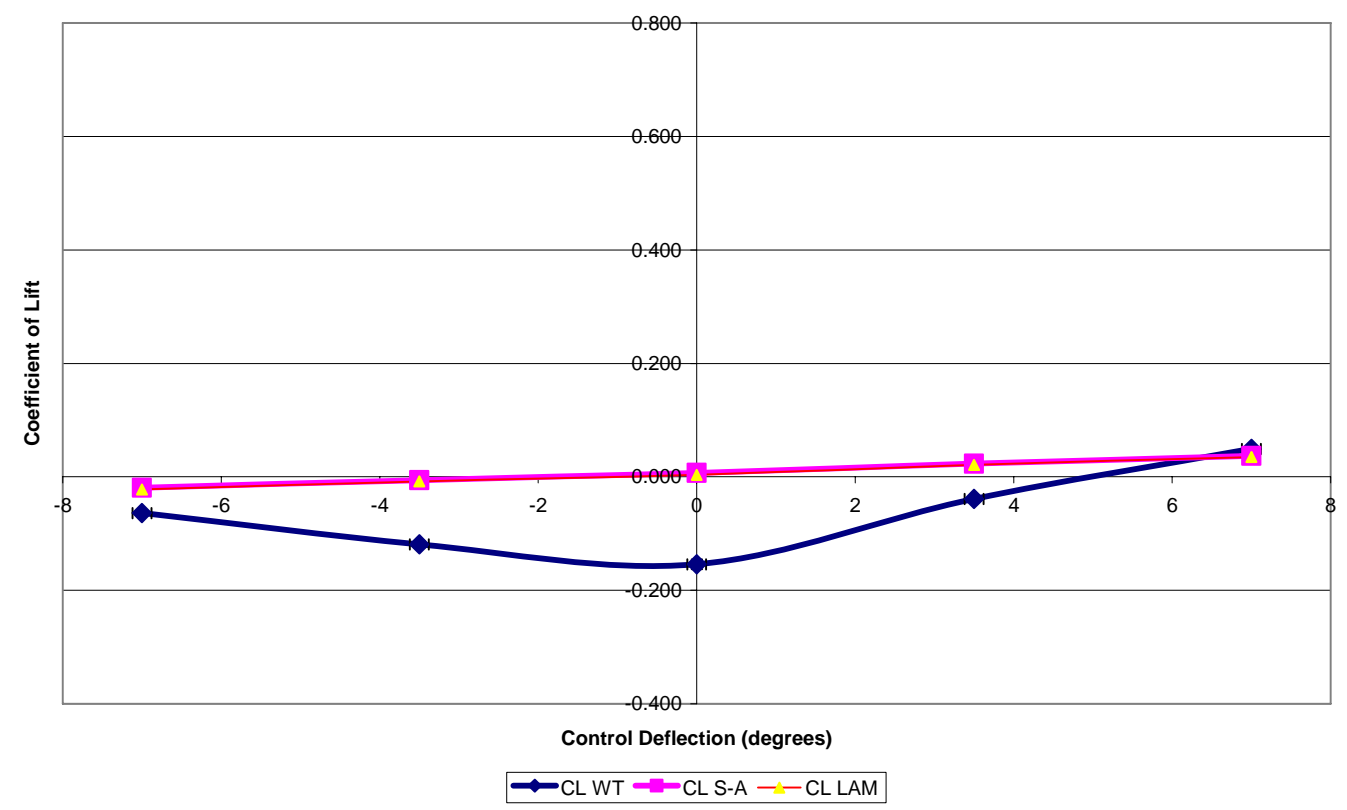

Figure 144 - Coefficient of Lift Comparison between wind tunnel data, a CFD model using SpalartAllmaras turbulence model and a Laminar flow CFD model at zero angle of attack 


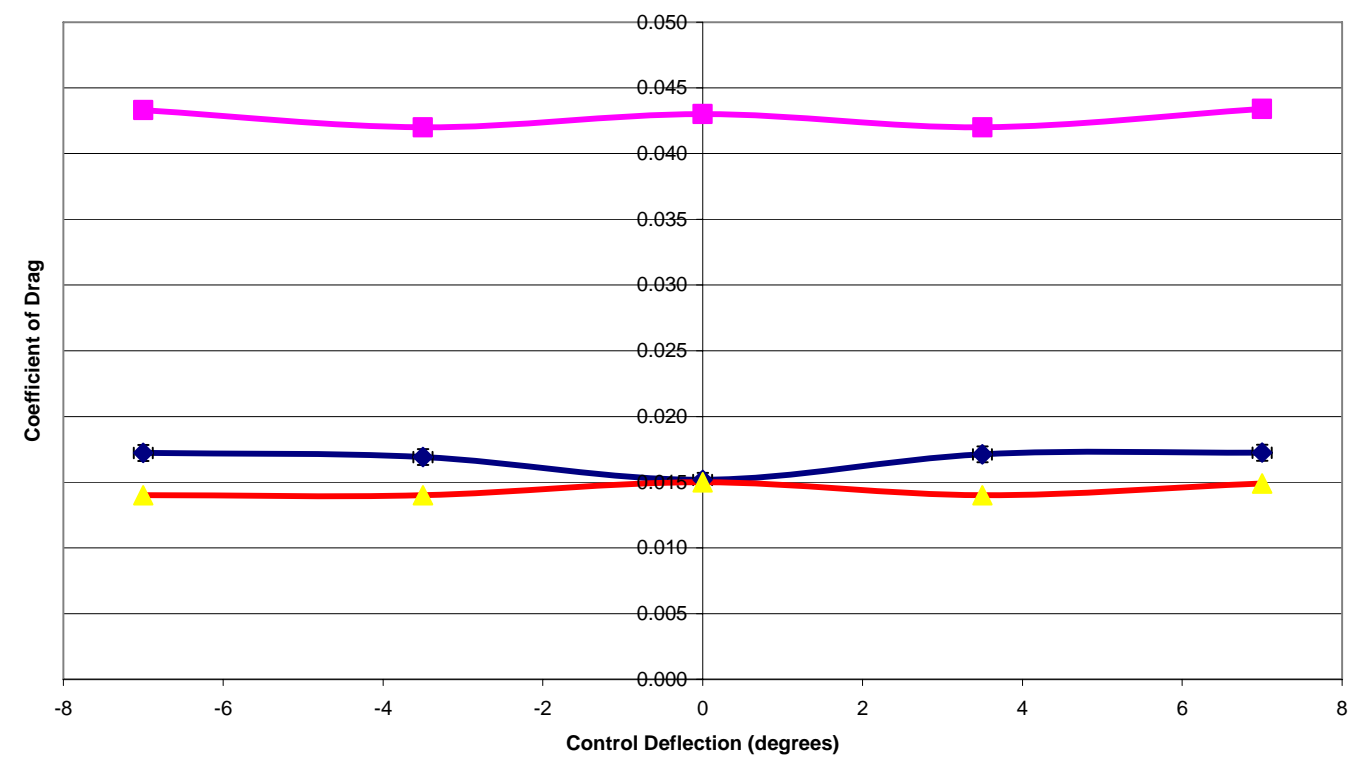

$\leadsto$ CD WT $2-$ CD S-A -- CD LAM

Figure 145 - Coefficient of Drag Comparison between wind tunnel data, a CFD model using SpalartAllmaras turbulence model and a Laminar flow CFD model at zero angle of attack.

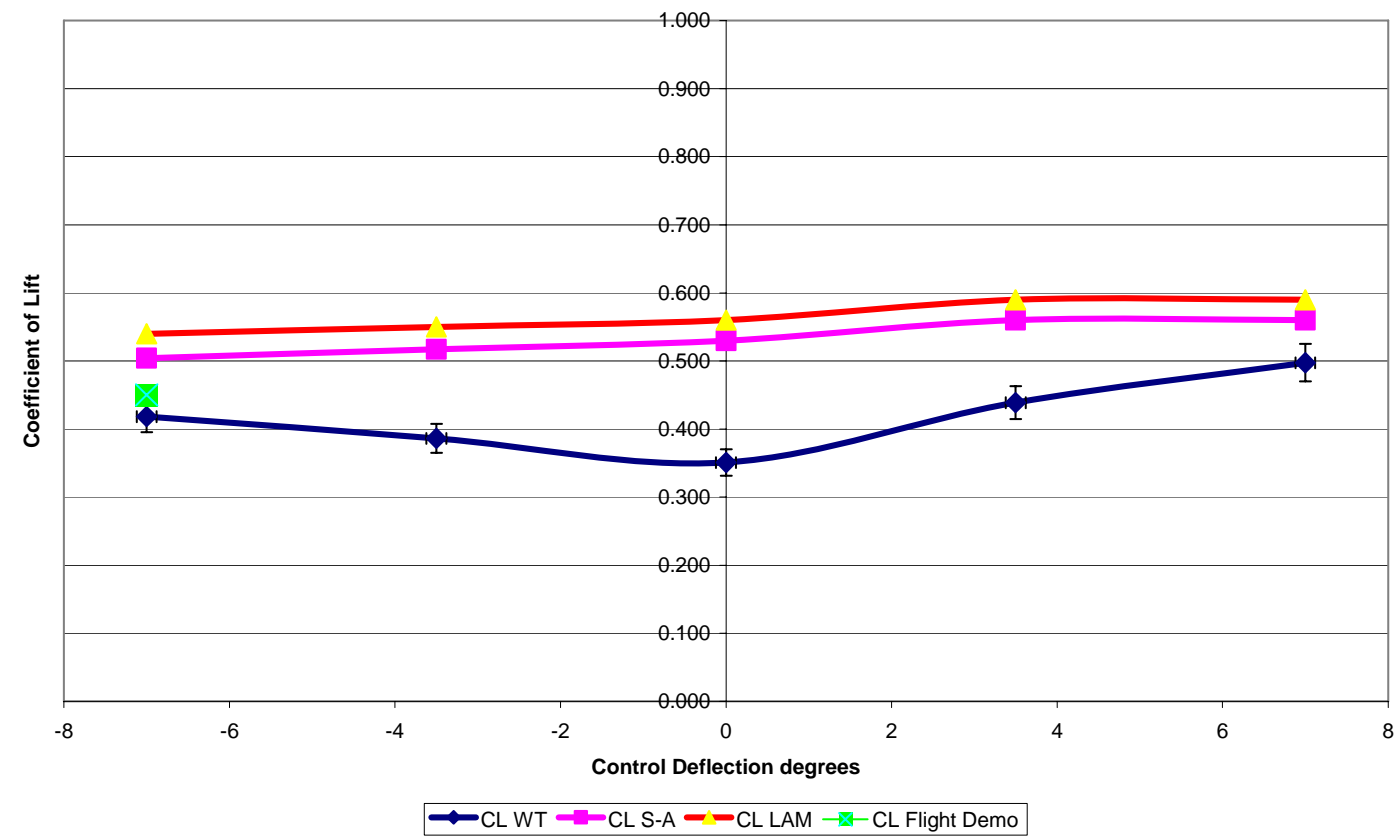

Figure 146 - Coefficient of Lift Comparison between wind tunnel data, a CFD model using SpalartAllmaras turbulence model and a Laminar flow CFD model at 7 degrees angle of attack. 


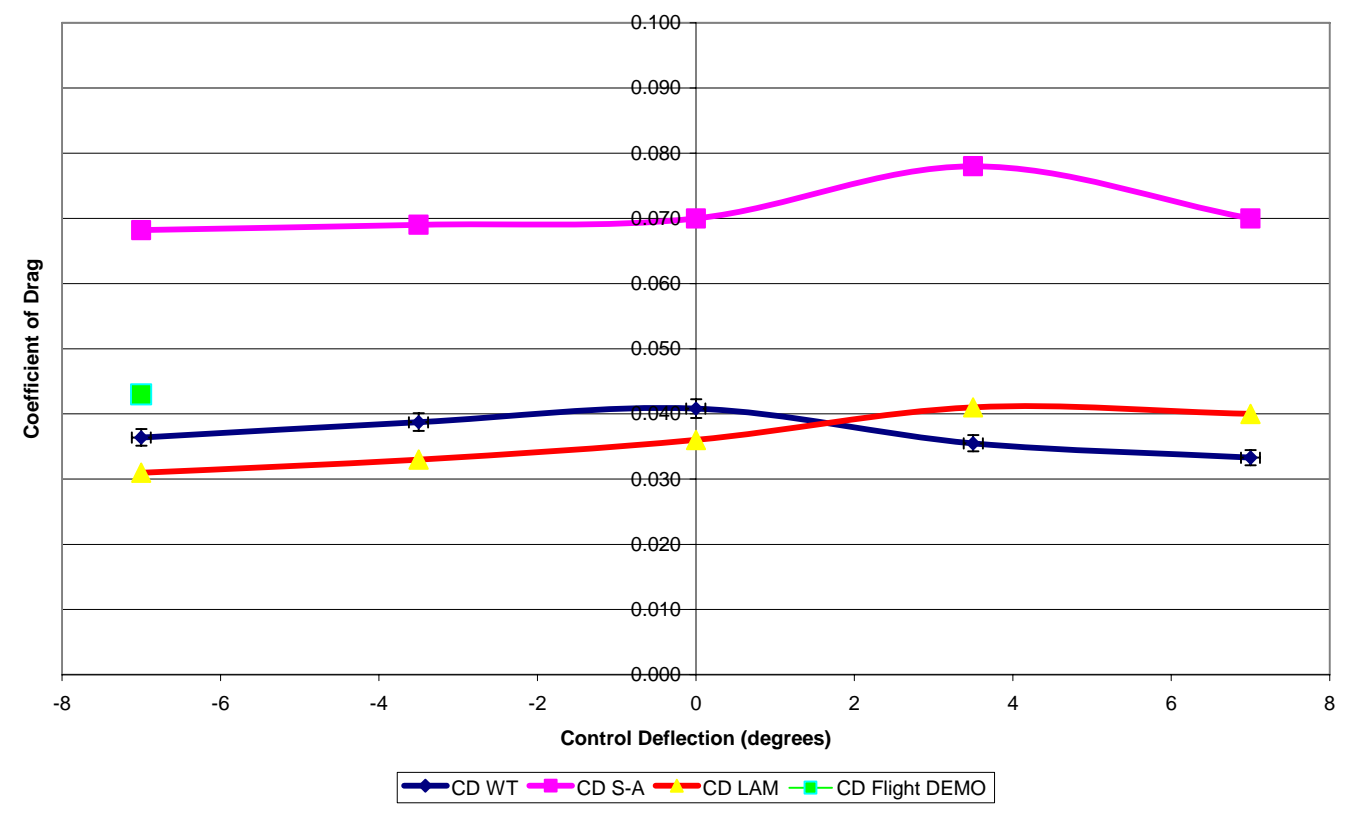

Figure 147 - Coefficient of Drag Comparison between wind tunnel data, a CFD model using SpalartAllmaras turbulence model and a Laminar flow CFD model at 7 degrees angle of attack.

At zero degrees angle of attack the lift coefficient of the wing stays around zero for all three data sets. Both the laminar and turbulent models are in good agreement, but both have higher lift values than the wind tunnel model's data. The laminar CFD model's coefficient of drag values match wind tunnel data very well in magnitude but have their highest value at the neutral control position, where as the wind tunnel data has this value at the extreme $+/-7$ degree control deflections. The Spalart-Allmaras turbulent model greatly over predicted $C_{D}$. Drag values varied little at zero AOA for any data set. The wind tunnel and laminar models varied between 0.014 and 0.017 and the turbulent model varied between 0.042 and 0.043 .

Lift coefficients at 7 degrees AOA, for both data sets, as well as the flight demonstrator's approximate data show good agreement in magnitude and general behavior except that the wind tunnel models shows a decrease in lift at the neutral control position that the others do not show. The CFD models show an increase in lift from the -7 degree control deflection through the +7 degree control deflection. If just the extreme control deflections in the wind tunnel data are considered the wind tunnel data would have very similar behavior to the CFD models. The CFD model's $\mathrm{C}_{\mathrm{L}}$ values are higher than the wind tunnel's at between 0.51 to 0.59 compared to 0.41 to 0.49 for the wind tunnel. Drag value magnitudes show good agreement between the laminar CFD model and the wind tunnel data, while the Spalart-Allmaras model over predicted $C_{D}$. All three models show an increase in $C_{D}$ from the -7 degree control position to the neutral control position. Then both the CFD models show $\mathrm{C}_{\mathrm{D}}$ increasing to the +3.5 degree control deflection then heading down again while the wind tunnel data makes this downward turn just before the +3.5 degree control position. 
The flow physics which cause the drag behavior present in the 7 degree AOA wing between the neutral and +7 degree control deflections is difficult to study. In general the CFD models show the low pressure region on the wings top surface extending toward the wing tip, intensifying and rotating toward the leading edge with increasing control deflection. The high pressure air below the wing has a similar trend except that it reaches the wing tip before the +3.5 degree control position and then retreats again between +3.5 and +7 degree control positions, which may indicate that it is bleeding off the trailing edge before the tip. At the +3.5 degree control position the high pressure air covers most of the tip and has a very steep gradient. This may indicate that it is feeding the tip vortex. Pressure profiles at the tip indicate that the strongest tip vortex is at the +3.5 degree control position and then rapidly breaks up between +3.5 and +7 . This breakup corresponds to a slight decrease in drag without any degradation on lift between these two control positions. This tip vortex breakup can clearly be seen in Figure 148

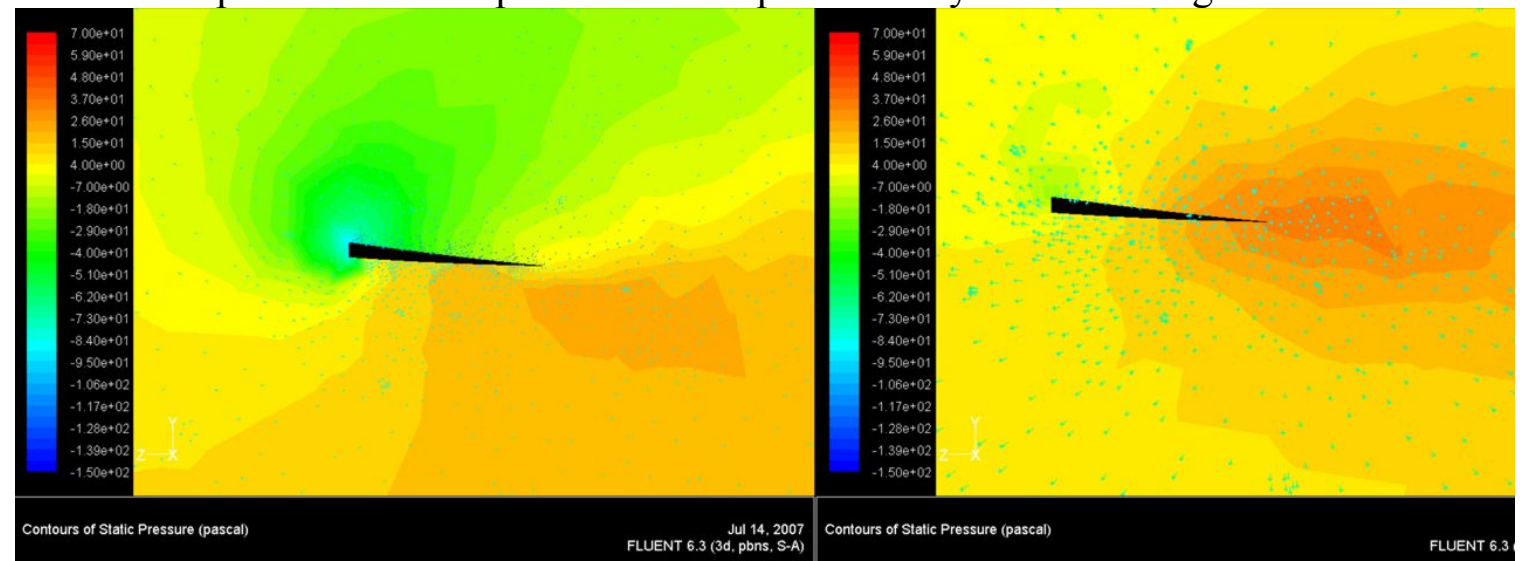

Figure 148 - Low pressure core structures at the wing tip at the +3.5 degree control deflection (left) and the +7 control deflection (right).

Observations made on the trailing edge flow from the morphing wind tunnel model with polyester tufts showed an apparent loss of the tip vortex at a control deflection between 0 and -3.5 degrees which is not seen in the computer models. The tufts also showed an increase in RPM of the tuft at the wing tip as the control deflection increased toward +3.5 degrees, but before reaching 3.5 degrees it began to slow all the way until the +7 degree control position. It also must be mentioned that the wake flow structures observed in both the wind tunnel models and in the CFD models were all very weak. In the wind tunnel it was almost impossible to observe them with smoke and in the CFD models any sign of structure like tip vortices are gone after going 2 or 3 inches downwind of the model. 


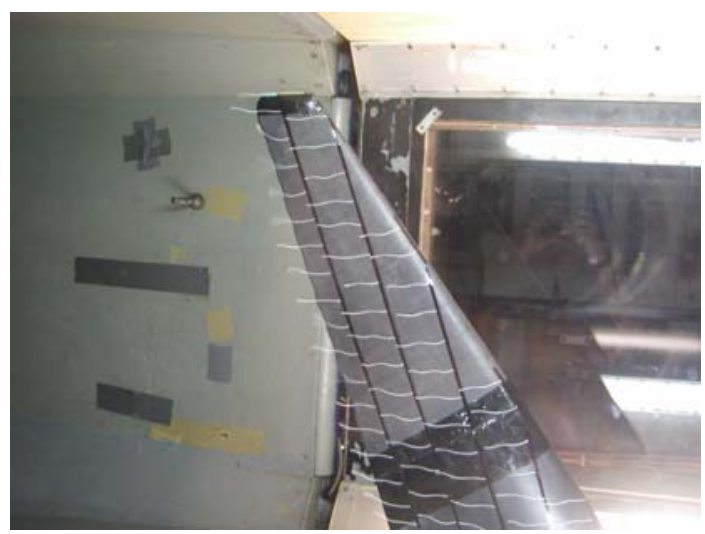

Figure 149 - Morphing Wing at $20 \mathrm{~m} / \mathrm{s}, 7$ degree AOA and control position of approximately -2 degrees. Note the apparent lack of tip vortex.

Smoke flow visualization in the wind tunnel showed an unusually behaving upwash at the wing tip which tended to adjust itself to be in a constant angle range to the wing tip with a control deflection. Both sets of CFD data show the same phenomena as can be seen in Figure 149 and Figure 150 where the tip has a control deflection of +7 degrees and the wing has a +7 degree angle of attack which give the tip +14 degree angle of attack to the tunnel flow yet the localized upwash changes this to be +7 degrees or less. The upwash can be seen up to 4 tip cord lengths forward of the wing in certain conditions. The self adjusting pressure field which is responsible for the upwash may be a factor in the pilot's inability to get the flight demonstrator to go into a tip stall.

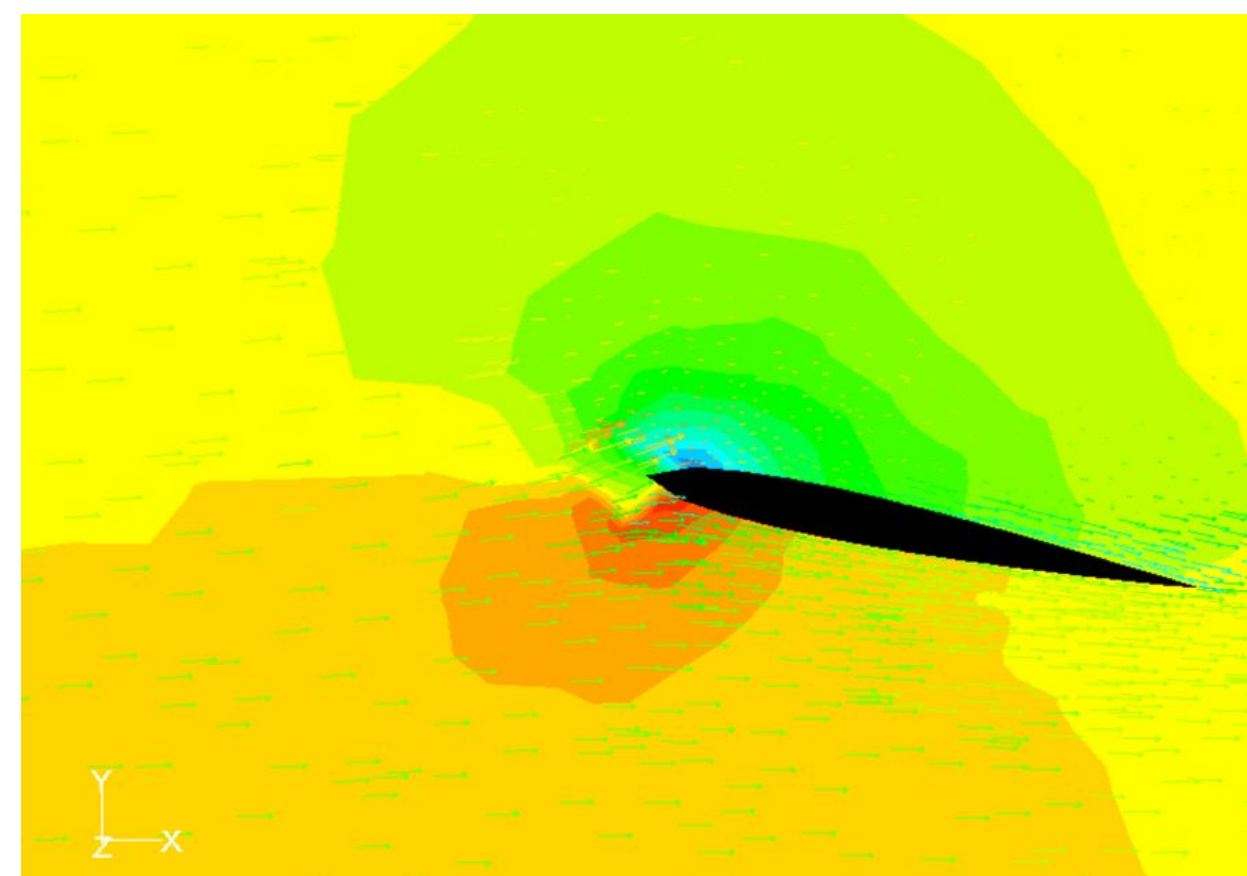

Figure 150 - Morphing Wing Tip 7 degree AOA and control position of +7 degrees or +14 degrees to the wind tunnel flow, yet regionally this angle is much less due to upwash. 
There are two factors which may contribute to the slight increase in lift and decrease in drag when approaching the +7 degree control deflection. One is the breakup of the tip vortex and the other is the rotation of the extreme low pressure from the wing top to the outboard $1 / 3$ of the wing's leading edges, which may develop a pressure distribution which produces induced thrust at the wings tip. The unique flow and pressure distributions are caused by the combination of a large high lift root, the 35 degree sweep and the 4 degrees of dihedral. It is likely that this very unique set of conditions would be hard to maintain in different velocity or Reynolds number ranges.

When the flow and pressure from the laminar CFD model is studied, on the wing surface, in the tip region, at a 7 degree angle of attack and at the +7 degree control position, unusual low pressure structures can be seen. Static pressure plots of the wing surface show valleys of low pressure which progress from the leading edge and curve toward the tip trailing edge. These low pressure valleys intersect the low pressure region which follows the leading edge to create scallop pattern along the leading edge. (Figure 151) This scallop pattern was also seen in the tempura paint flow visualization, but was disregarded at that time because of a lack of understanding. When velocity magnitude vectors just above the tip surface are overlaid on the pressure contours, it becomes obvious that rotational flow exists at the center of each of these scallops and the low pressure valleys which extend from them. These features appear to be vortex filaments leading from the leading edge. (Figure 152)

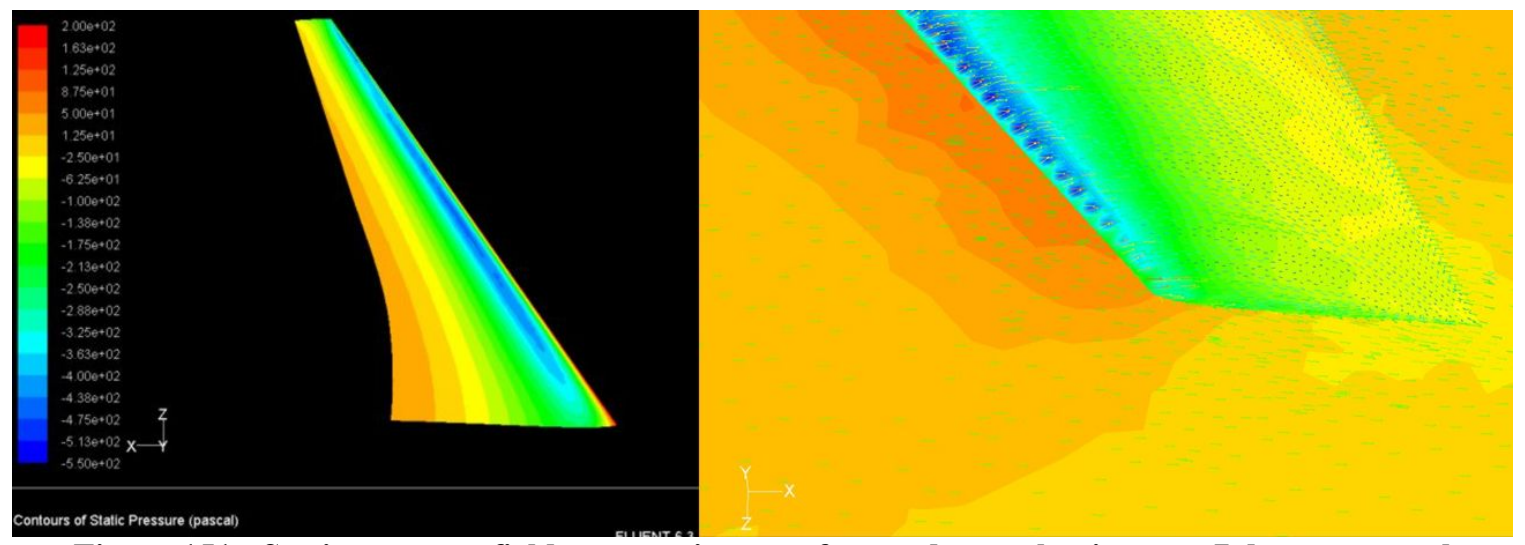

Figure 151 - Static pressure field on top wing surpface and near the tip at a +7 degree control deflection with the wing at 7 degrees angle of attack. 


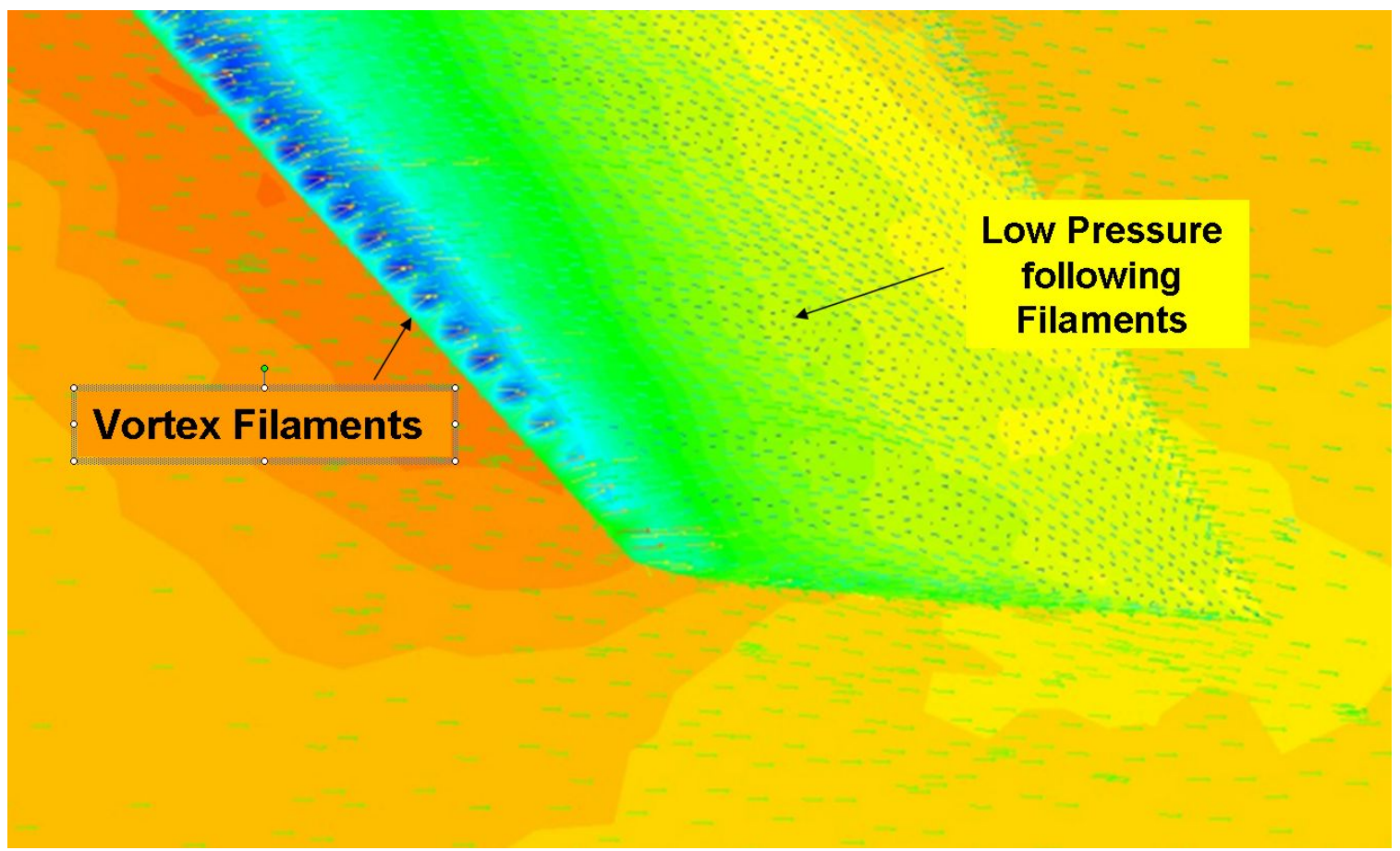

Figure 152 - Static pressure and velocity magnitude flow vectors just above the wing tip surface. 


\section{Conclusions}

This research project developed an adaptive washout morphing mechanism for the control of a swept wing tailless aircraft. The adaptive washout morphing mechanism was able to provide effective roll, yaw and pitch control for a swept wing tailless aircraft. This new control technique was experimentally and numerically compared to an existing elevon equipped tailless aircraft and has shown the potential for significant improvements over that system in terms of efficiency, better lift/drag. The feasibility of this mechanism was also validated by designing, fabricating and flight testing a flight weight version which performed in much the same way that conventional elevon controls do.

In the process of comparing the Horten type elevon equipped models and the morphing models, formal wind tunnel verification of wingtip induced thrust found in Horten (Bell Shaped Lift distribution) type swept wing tailless aircraft was documented. This was exploited by the Horten's to minimize adverse yaw and drag. The extreme downward elevon deflection results in the forward rotation of tips lift vector, which counters adverse yaw (ie. Reduced drag at the wing tip). Wind tunnel data also indicates that this effect may only exist in a small velocity range for a given built in wing twist geometry. This would agree with the Horten test pilot's observation of problematic adverse yaw during many maneuvers.

Through the use of wind tunnel testing, flow visualization and computational fluid dynamic (CFD) studies a more developed physical understanding of the highly complex flow developed in the control region of the morphing tailless aircraft has been developed. The data collected shows physical reasons for the phenomenon observed in the morphing model; of the control position which has the highest CL also has the lowest CD. The are two factors which run in parallel to this phenomena, 1) The rotation of low pressure air toward the leading edge of the tip of the wing when the tip is at a +7 degree control position, 2) the break up of tip vortex when the controls are between $a+3.5$ degree and a +7 degree deflection.

CFD models indicate the possibility of the presence of a Leading Edge Vortex (LEV) on the control section morphing wing when the tip is twisted between +3.5 degrees and +7 degrees. The presence of this LEV may be the cause of the reduction of drag while lift is increasing. Similar LEVs have been documented in use by birds and insects. A 2004 study by Videler, Stamhuis and Povel in Holland was trying to uncover the flow physics responsible for the unusual maneuvering abilities the common swift. This study used detailed wing models in a water tunnel, particle image velocimetry and observation of the common swift in flight. The study uncovered the swift's use of LEVs in flight. The swift is able to adjust both sweep and twist in order to maintain or harness LEVs at angles of attack of between 5 and 10 degrees. These abilities are made possible by the unique geometry of the swifts wing. The swifts wing is separated into two parts, the arm wing, near the body, and the hand wing, toward the tip. The arm wing has a relatively thick airfoil section with a large rounded leading edge and the hand wing has a much thinner profile with a sharp leading edge. (Figure 153) This unique geometry along with wing 
sweep and the ability to twist the wing in flight is not seen in manned aircraft, but the morphing wind tunnel models and the morphing flight demonstrator posses very similar features and fly in a similar Reynolds number range to the common swift. These specific flow physics appear to be Reynolds number dependent.

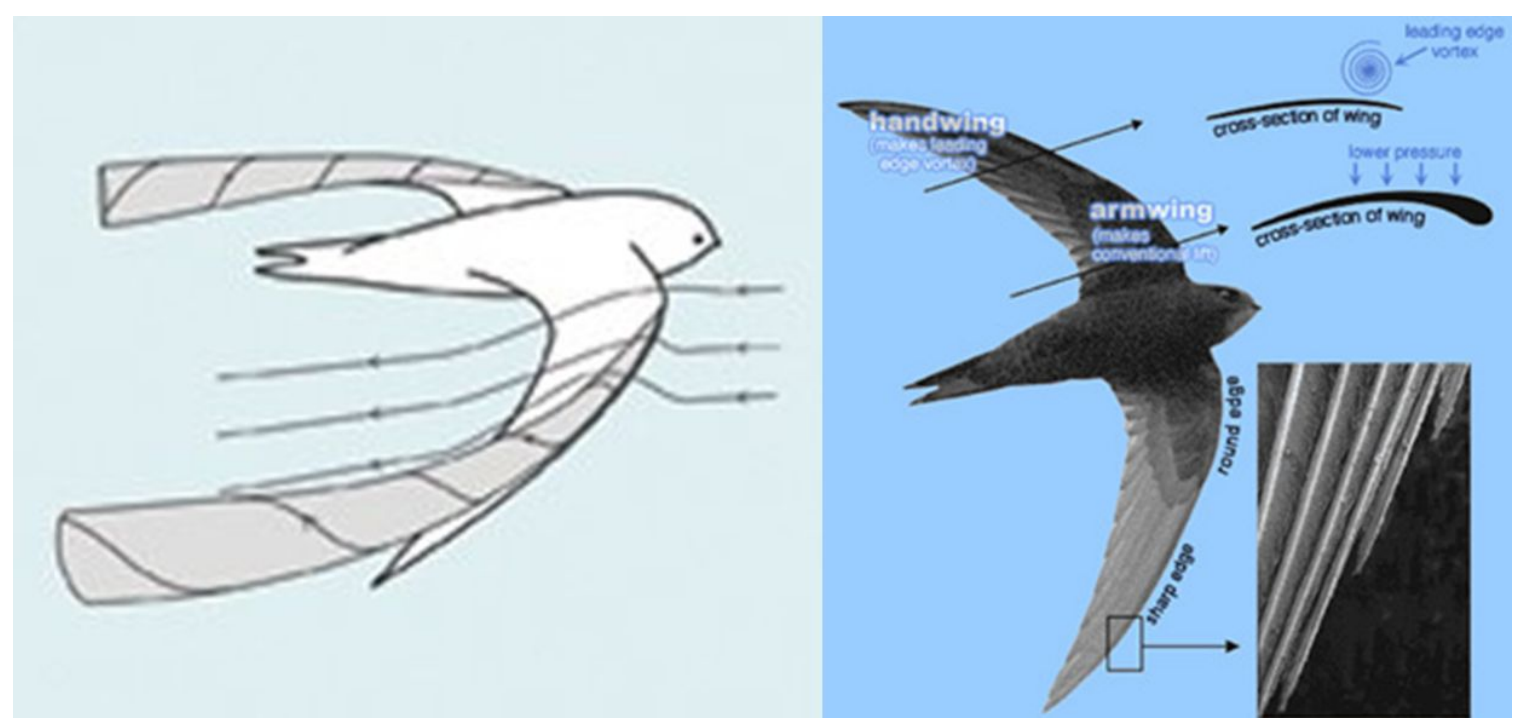

Figure 153 - The common swifts use of LEVs and the swifts wing geometry. (Weiss, 2004)

This research also has given a rare comparison of Low Reynolds number CFD data, wind tunnel data, wind tunnel flow visualization and free flight data. The exact same geometry and Reynolds number was used in all and the results agreed well with each other in the narrow range of test conditions.

The viability and potential for efficiency gains have been documented and further research should be conducted in order to further characterize this type of control and to explore the possibilties of using both varable sweep and adaptive washout to harness LEVs in low Reynolds number unmanned aircraft flight. 


\section{Bibliography}

Akedemische Fliegergruppe Braunschweig, "The Akaflieg Braunschweg SB-13 Project, http://www.tu-bs de/studenten/akaflieg/English/ProjectSB13/ProjectSB13/main.html, 2003.

Berns, Hans-Jürgen, "Flight Testing the SB-13 Flying Wing", SETP Presentation, April 1997,http://www.tubs_de/studenten/akaflieg/English/SB13FlightTest/FTSB13ProjectSB1 3/main.html, 2003.

Bowers, Albion, “The Horten HX Series: Ultralight Flying Wing Sailplanes”, TWITT Presentation, El Conjon CA, USA, Sept. 19, 1998.

Bowers, Albion, "Flying Wing Aerodynamics", Internet Forum http://yarchive.net/mil/flying_wing.html, April 1999.

Bowers, Albion, and Lednicer, D. A., "A retrospective: Flying Wing Design Design" Presentation for the The Wing is The Thing Organization, 20 September 1997, http://www.nurflugel.com, 2003.

Bowmen, J., Sanders, B., and Weisshaar, T., "Morphing Aircraft, What they are, What do we do with them”, Presentation for the AFRL / Air Vehicles Directorate 1997.

Brenner M.J., Lind R. C., and Voracek, D. F., "Overview of Recent Flight Flutter Testing Research at NASA Dryden”, NASA Technical Memorandum 4792, April 1997.

Bullard, D., "Nurflugel", website devoted to tailless aircraft, http://www.nurflugel.com, 2001-2005.

Burger, D., "New Ideas on Bird Flight" Presentation about Dr. Burgers NATURE article given to TWITT organization, 20 March 1999.

Cadogan, D., Smith, T., Uhelsky, F., and MacKusick, M., "Morphing Inflatable Wing Development for Compact Package Unmanned Aerial Vehicles" AIAA paper AIAA 2004-1807, 2004.

Chow, J., Zilliac, G., and Bradshaw, P., "Turbulence Measurements in the Nearfield of a Wingtip Vortex," NASA Technical Memorandum 110418, February 1997.

Curry, Marty, "NASA AFTI/F-111 MAW Fact Sheet" NASA Dryden Flight Research Center, http://www.dfrc.nasa.gov/Gallery/Photo/F-111AFTI/HTML/EC88-0052-4.html, Dec. 2004.

Curry, Marty, "NASA AAW Fact Sheet FS-061-DFRC” NASA Dryden Flight Research Center, http://www.nasa.gov/centers/dryden/news/FactSheets/FS-061-DFRC.html, Feb. 2005. 
Fluent Inc., "Fluent User's Guide,” Fluent, 2006

http://www.fluentusers.com/fluent/doc/ori/html/ug/main pre.htm, October 15, 2006.

Gano, S. and Renaud, J., "Optimized Unmanned Aerial Vehicle with Wing Morphing for extended Range and Endurance", AIAA paper AIAA-2002-5668, 2002.

Garcia, H., M., Abdulrahim, M., and Lind, R., “ Roll Control for a Micro Air Vehicle Using Active Wing Morphing”, University of Florida, AIAA paper AIAA-2003-5347, 2003.

George-Falvy, Dezso, "A Review of Aerodynamic Evaluation Tests of the Horten IVa Flying Wing”, Presented at the National Soaring Museum Wing Symposium, Elmira, NY, July 17, 1997.

Hallenberg, Jonathan, "The Wright Brothers, the Invention of the Aerial Age", National Air and Space Museum, Smithsonian, http://nasm.si.edu, 2004.

Hepperle, Martin, “Airfoil Design for Light Tailless Airplanes”, http://www.mhaerotools.de/airfoils/nf_8.htm, 2004.

Horten, Reimar, "The Idea - translated by Yoram Lesshinski”, http://www.nurflugel.com/Nurflugel/Horten_Nurflugels/horten_nurflugels.html, 2003.

Horten, Reimar and Horten, Walter, "Ten Years Development of the Flying Wing HighSpeed Fighter", Excerpt from LGB-164 / Advance Report presented at Bonn Flying Wing Seminar, April 14, 1943, http://www.nurflugel.com, 2003.

Horten, Reimar, and Selinger, Peter, "Nürflugel-Die Geschichte der Horten-Flugzeuge 1933-1960”, H. Weishaupt Verlag, Graz,Austria, 1993.

Kim, S.-E., and Rhee, S.H., "Prediction of Tip-Vortex Flow Past a Finite Wing," 43rd AIAA Aerospace Sciences Meeting and Exhibit, Reno, USA, Jan 10-13, 2005.

Kikuta, M., "Mechanical Properties of Candidate Materials for Morphing Wings", Masters Thesis Virginia Polytechnic Institute - Mechanical Engineering Masters Thesis, Blacksburg, VA, December 2003.

Koopman, G., Lesieutre, G., Frank, J., and Loverich, J., "Design and Modeling of High Power Piezoelectric Actuator, Penn State University Presentation, http://kirkof.psu.edu/Banff\%202004/Loverich/Banff\%20Actuator\%20Presentation\%20\%20Jacob.ppt, 2004. 
Kressse, Art, "The Design, Construction and Flying of a Flying Wing”, http://www.nurflugel.com, 2003.

Kroo, Ilan, "Blended Wing Body Project" Stanford University website to describe BWB research, http://aero.Stanford.edu/BWBProject.htm, 2003.

Kroo, Ilan, Beckman, Eric, Robbins B., Morris, S., and Porter, B., "Development of the Swift - A Tailless Foot Launched Sailplane”, Hang Gliding Magazine, http://aero.stanford.edu/Reports/SWIFTArticle1991.html, Jan. 1991.

Kulhman, Bill and Kulhman, Bunny, "On The Wing - Flying Wing Design and Analysis, twist Distributions for Swept Wings, Part 3", Radio Controlled Soaring Digest, http://www.b2streamlines.com, Sept. 2002.

Lazos, Barry "Biologically Inspired Aerodynamics" Personal Correspondence, NASA LaRC, 2003.

Marks, Paul, "The next 100 years of flight - part two", Online magazine NewScientist.com news service, http://www.newscientist.com/news/print.jsp?id=ns99994484, December 13, 2003.

Meyers, J.F., Lee, J.W., Fletcher, M.T., and South, B.W., "Hardening Doppler Global Velocimetry Systems for Large Wind Tunnel Applications", NASA Langley Research Center Paper, Hampton, VA, 1992.

Miller, S. L., Meyers, J. F., and Usry, J. W., "Doppler Global Velocimetry: Apotential Velocity Measurement Method for General Aviation Applications" Presented at AIAA Second Joint Symposium on General Aviation Systems, Wichita, KS, March 16-17, 1992.

Mills, A.F.and Chang, B.H.,'Error Analysis of Experiments, A manual for Engineering students", University of California, 2004.

Mythra, David, "The Horten Brothers and their All-Wing Aircraft", Schiffer Publishing Ltd., Atglen, Pa, USA, 1998.

Nickel, Karl, and Wohlfahrt, Michael, "Tailles Aircraft in Theory and Practice", American Institute of Aeronautics and Astronautics, Inc. Washington, D.C., 1994.

Northrop, John, K., "The Development of All-Wing Aircraft" $35^{\text {th }}$ Wilbur Wright Memorial Lecture, London, May 29, 1947.

Schäfer, Ulrich, “Aachen” Todays Pilot, Key Publishing April 2002.

Simpson, J. O., Wise, S. A., Bryant, R. G., Cano, R. J., Gates, T. S., Hinkley, J. A., Rogowski, R. S., and Whitley, K. S., "Innovative Materials for Aircraft Morphing", 
NASA Langley Research Center Aircraft Morphing Program Report, Hampton, VA, 1999.

Smith, H., "Blended Wing Body Airliner - the next generation of civil transport aircraft Preliminary Design Study BW-98, Cranfield College of Aeronautics, http://www.cranfield.ac.uk, 2003.

Stadler, R., "Some Comments on the Bell Shaped Lift Distribution" http://www.nurflugel.com, September, 1998.

Stanford University Department of Aeronautics "Tailless Aircraft" Web based textbook, http://www.desktopaero.com/appliedaero/configuration/tailess.html, 2003.

TWITT, "Rod Schapels SA-882 Flying Wing Prototype", The wing is the thing rganization, http://members.cox.net/twitt/schapel.htm, 2003.

Weiss, Peter, "Swift Lift - Birds may get a rise out of swirling air" Science News, Vol. 166, No. 24, p373, Dec. 11, 2004.

Weisshaar, T., and Sanders, B., Purdue / AFRL Aircraft Morphing Project...one step closer" Presented to the Air Force Research Labs on March 27, 2001.

Wakayama, Sean, and Kroo, Ilan, “ The Challenge aqnd Promise of Blended Wing Optimization" AIAA paper 98-4736, 1998

Whitlock, Jennifer, "Blended Wing Body: Competitors and Other Flying Wings", http://www.geocities.com/witewings/bwb/competitors.html, June 2002.

Whitmer, C. E. , "Modeling and Control of a Morphing Airfoil Structure", Masters Thesis for Iowa State University Mechanical Engineering, Ames Iowa, 2003.

Wilkinson, K. G., "Farnborough Hants - The Horten Tailless Aircraft", http://members.cox.net/rebid/Farnborough_08.html, 2003.

Yen, Dora and Braeuchle, Frank, "Calibration and Uncertainty Analysis for the UC Davis Wind Tunnel Facility", UCD Wind Tunnel Calibration Document, May, 2000, http://windtunnel.engr.ucdavis.edu/calibration/Calibration_2.1.PDF, 2005.

\section{CONTACT}

Richard Guiler

rguiler@aol.com, rguiler@aurora.aero

Aurora Flight Sciences

3000 Benedum Industrial Dr

Bridgeport WV, 26330

$304-842-8100$ 


\section{Appendix A - Force Balance Data}

Drag Calibration

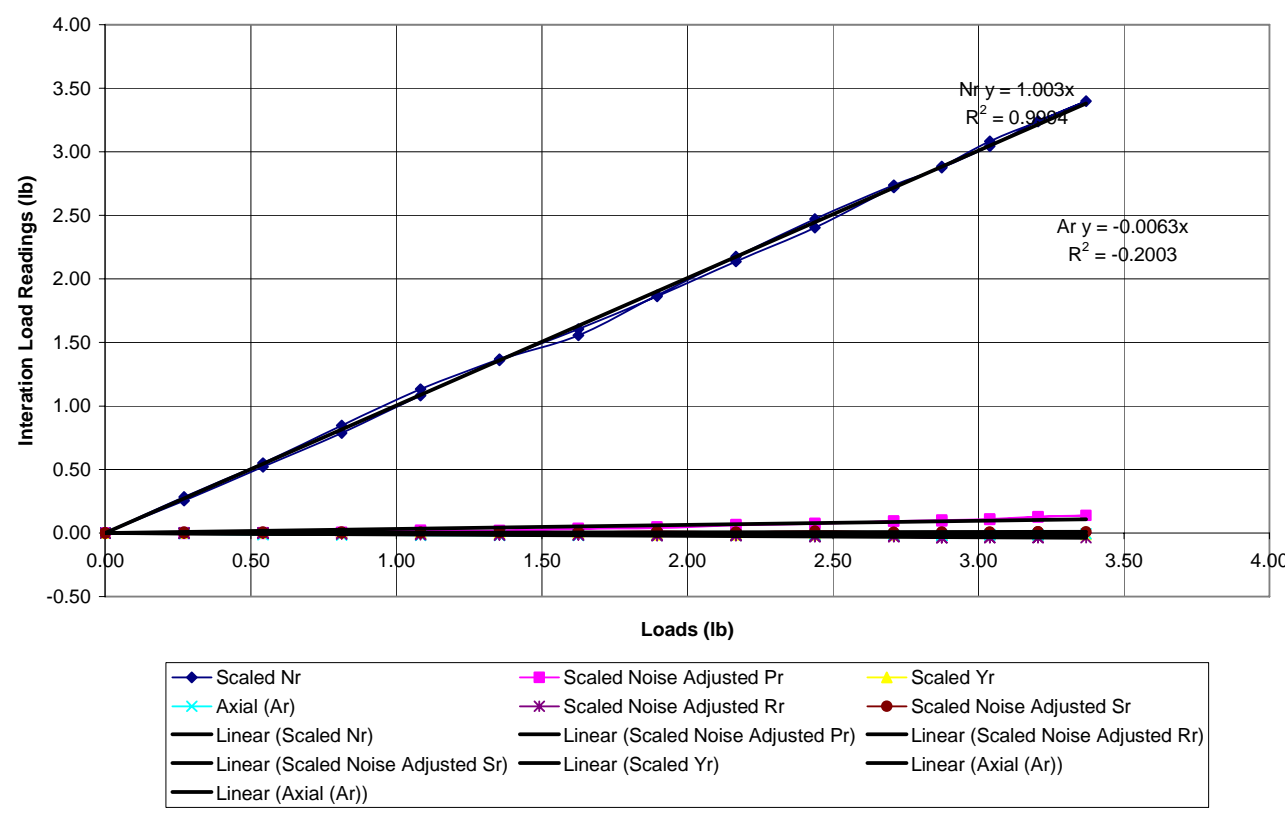

Figure 154 - NASA Balance \#826 Calibrations, Drag Component

Vertical Calibration

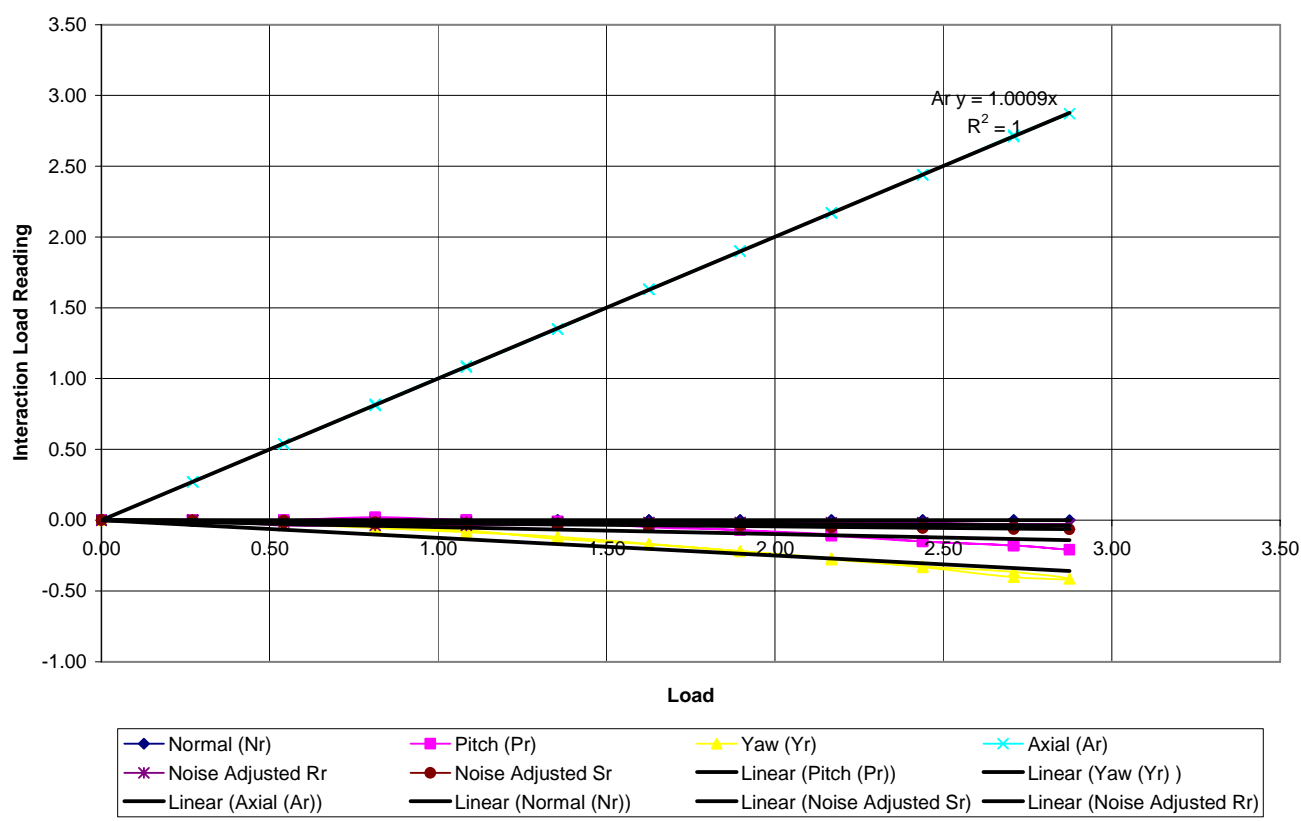

Figure 155 - NASA Balance \#826 Calibrations, Vertical Component 
Lift Calibration

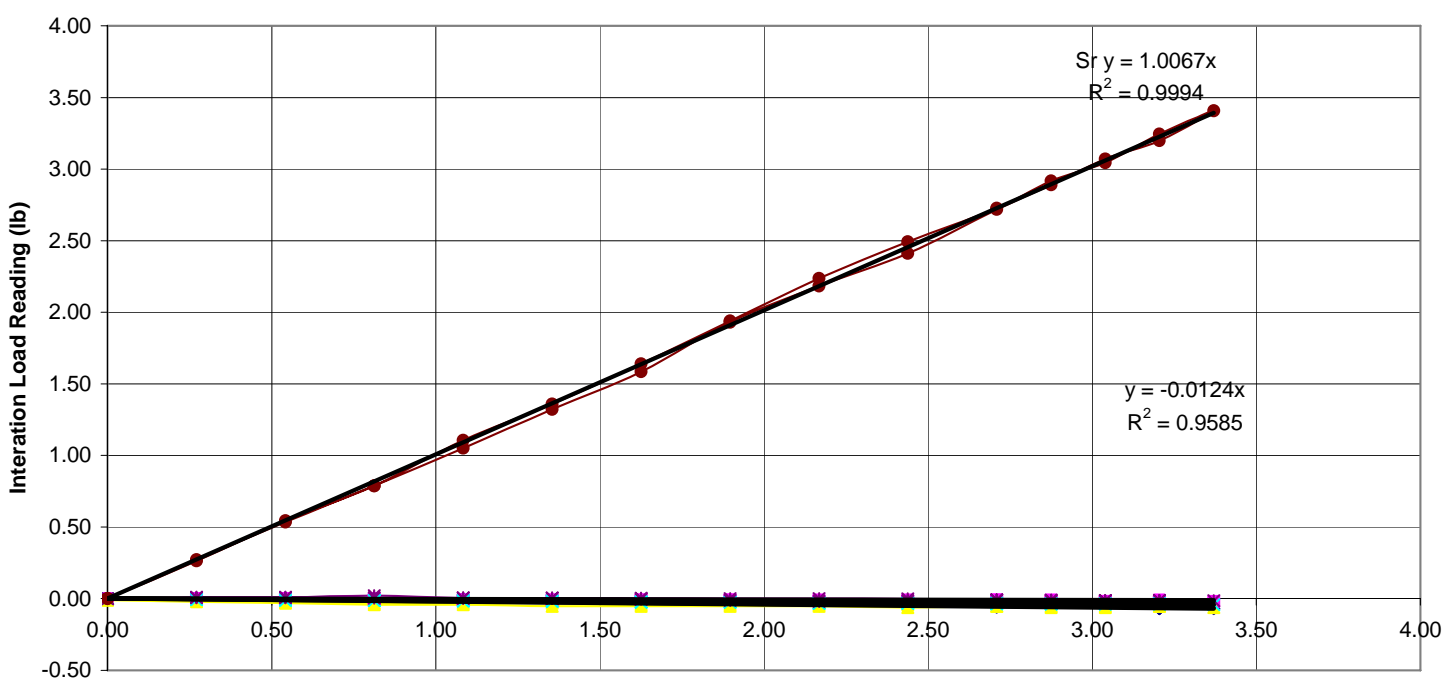

Load (lb)

\begin{tabular}{|c|c|c|}
\hline$\rightarrow \bullet$ Scaled $\mathrm{Nr}$ & - -Scaled Pr & Scaled Yr \\
\hline$\rightarrow$ Axial (Ar) & *-Scaled Noise Adjusted Rr & $\rightarrow-$ Scaled Sr \\
\hline —Linear (Scaled Sr) & —Linear (Scaled Nr) & —Linear (Scaled Pr) \\
\hline - Linear (Scaled Yr) & —Linear (Axial (Ar)) & —Linear (Scaled Noise Adjusted Rr) \\
\hline —Linear (Axial (Ar)) & & \\
\hline
\end{tabular}

Figure 156 - NASA Balance \#826 Calibrations, Lift Component

\section{Roll Calibration}

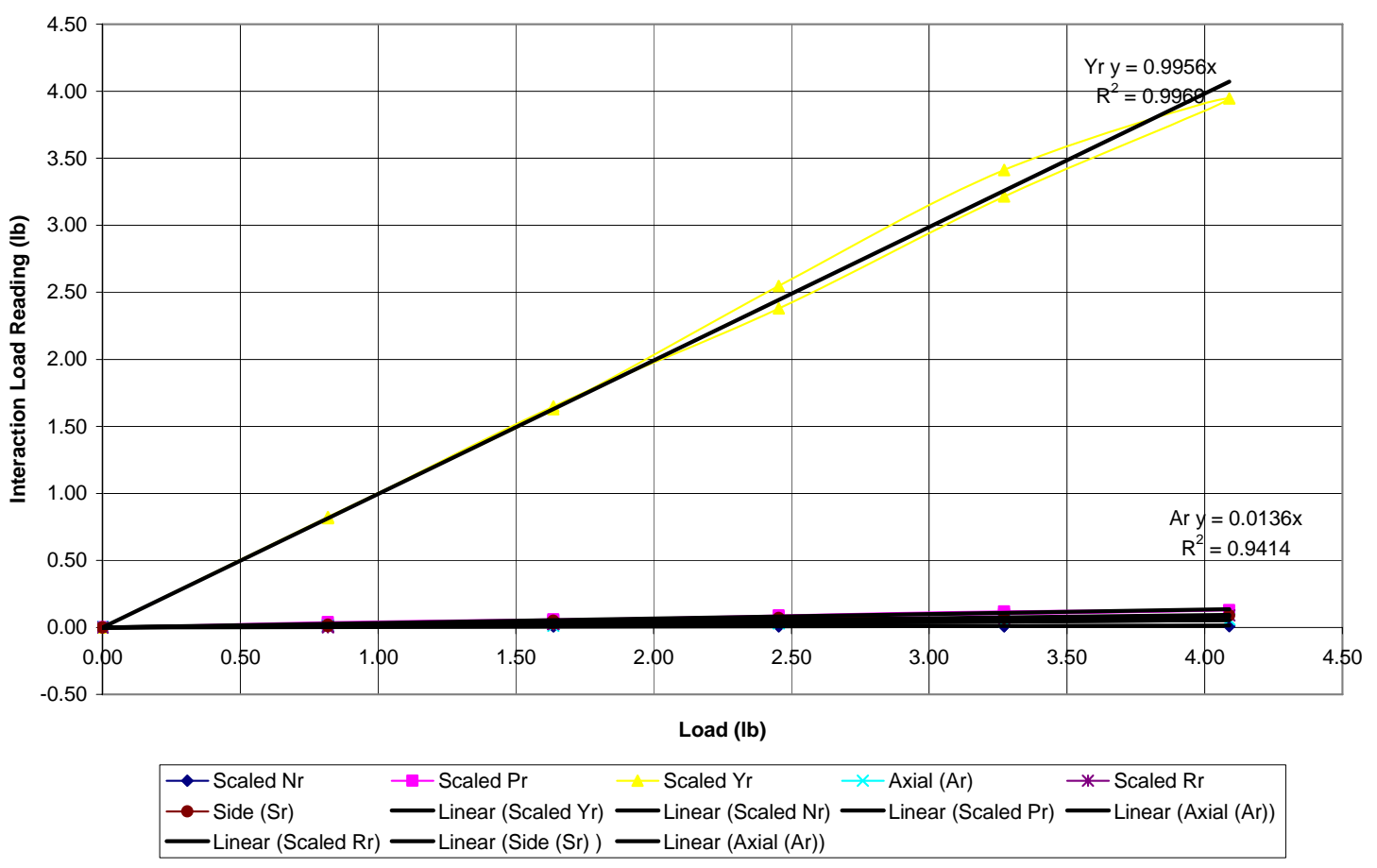

Figure 157 - NASA Balance \#826 Calibrations, Roll Component 


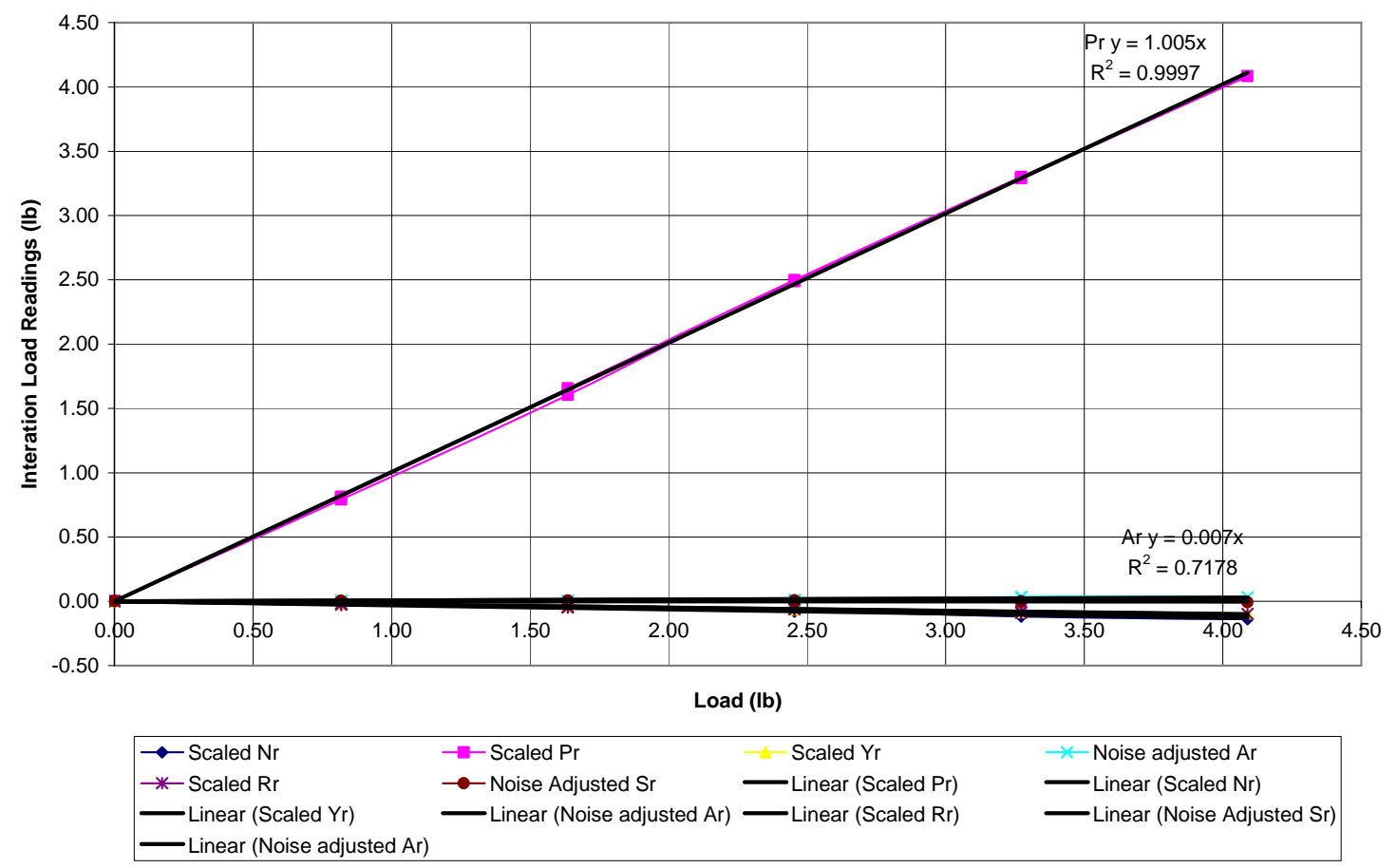

Figure 158 - NASA Balance \#826 Calibrations, Yaw Component

\section{Pitch Calibration}

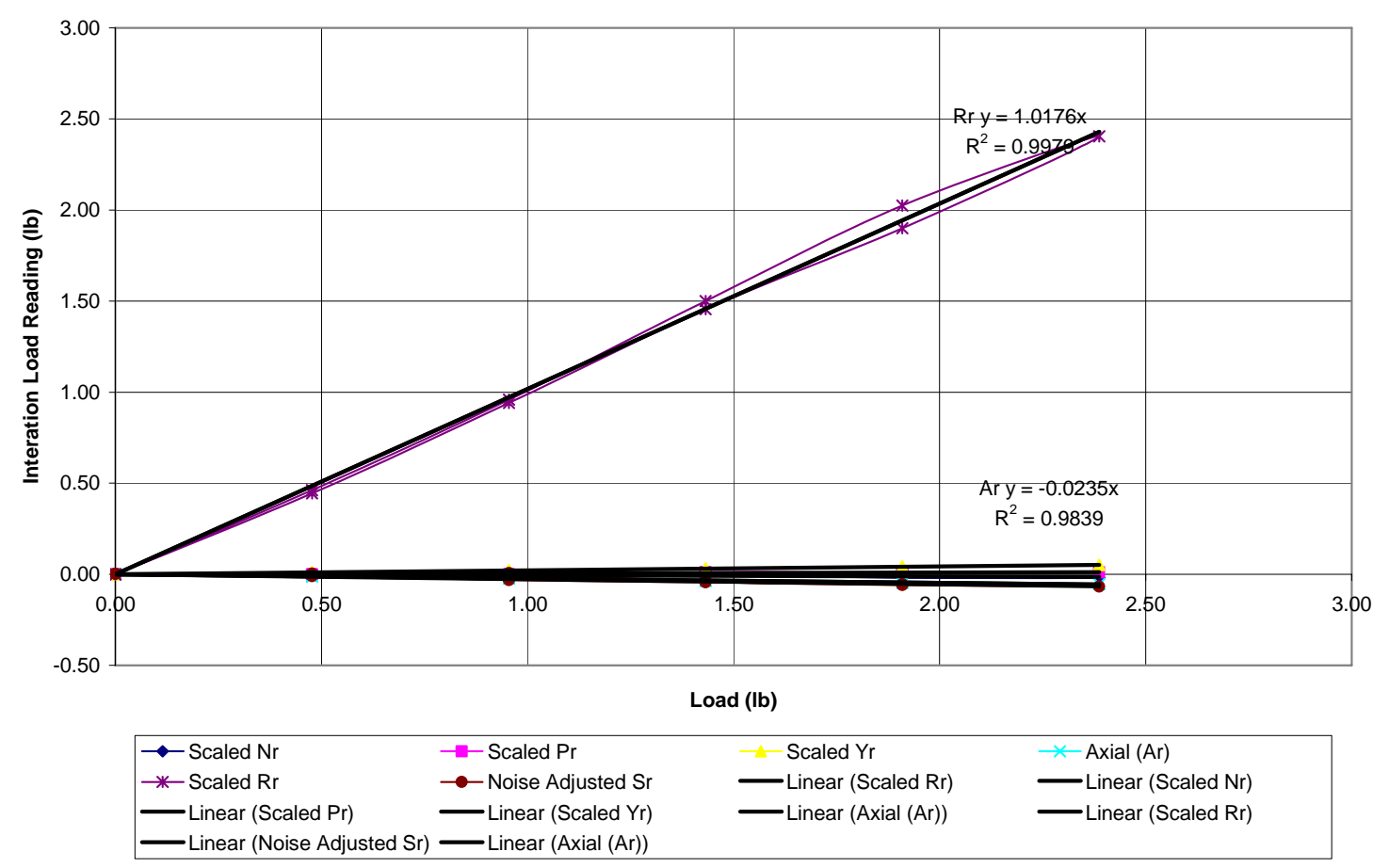

Figure 159 - NASA Balance \#826 Calibrations, Pitch Component 


\section{Appendix B - Wind Tunnel Data}

Table 17 - Aerodynamic Coefficient Data for Elevon Equipped Windtunnel Model in WVU Wind Tunnel 2006-2007

Elevon Wing, 0 degree

AOA, $10.628 \mathrm{~m} / \mathrm{s}$ TO18ET022E

0.8719

Control Position

\begin{tabular}{|c|c|c|c|c|}
\multicolumn{1}{c}{ CD } & CmY & CmP & CmR & CL \\
\hline 0.028 & 0.008 & 0.116 & -0.071 & -0.162 \\
\hline 0.026 & 0.008 & 0.157 & -0.101 & -0.231 \\
\hline 0.027 & 0.006 & 0.186 & -0.125 & -0.278 \\
\hline 0.026 & 0.010 & 0.101 & -0.065 & -0.163 \\
\hline 0.030 & 0.009 & 0.062 & -0.035 & -0.100 \\
\hline
\end{tabular}

L/D

$-5.878$

$-8.862$

3.5

0

$-3.5$

$-7$

\begin{tabular}{l|l}
0.030 & 0.009 \\
\hline
\end{tabular}

$-10.111$

$-6.369$

$-3.377$

Elevon Wing, 0 degree AOA, $15.94 \mathrm{~m} / \mathrm{s}$ T023ET028E

Control Position

7

3.5

0

$-3.5$

$-7$

\begin{tabular}{|c|c|c|c|c|}
\multicolumn{1}{c}{ CD } & \multicolumn{1}{c}{ CmY } & CmP & CmR & CL \\
\hline 0.014 & 0.012 & 0.129 & -0.082 & -0.202 \\
\hline 0.011 & 0.010 & 0.161 & -0.104 & -0.244 \\
\hline 0.013 & 0.008 & 0.172 & -0.107 & -0.241 \\
\hline 0.022 & 0.010 & 0.051 & -0.022 & -0.080 \\
\hline 0.019 & 0.022 & 0.199 & -0.113 & -0.278 \\
\hline
\end{tabular}

L/D

$-14.430$

$-22.407$

$-18.997$

$-3.550$

$-14.451$

Elevon Wing, 0 degree AOA, $21.25 \mathrm{~m} / \mathrm{s}$ T028ET032E

\section{Control Position}

7

3.5

0

$-3.5$

$-7$

\begin{tabular}{|c|c|c|c|c|}
\multicolumn{1}{c}{ CD } & CmY & CmP & CmR & CL \\
\hline 0.007 & 0.011 & 0.137 & -0.083 & -0.196 \\
\hline 0.005 & 0.009 & 0.163 & -0.100 & -0.225 \\
\hline 0.014 & 0.012 & 0.072 & -0.036 & -0.104 \\
\hline 0.019 & 0.011 & 0.046 & -0.018 & -0.074 \\
\hline 0.024 & 0.011 & 0.043 & -0.017 & -0.078 \\
\hline
\end{tabular}

L/D

$-29.145$

$-41.034$

$-7.263$

$-3.970$

$-3.191$

Elevon Wing, 3.5 degree AOA, $10.628 \mathrm{~m} / \mathrm{s}$ TO33ET037E 
Control Position

7

3.5

0

$-3.5$

$-7$

\begin{tabular}{|c|c|c|c|c|}
\multicolumn{1}{c}{ CD } & \multicolumn{1}{c}{ CmY } & CmP & CmR & CL \\
\hline 0.041 & 0.005 & -0.028 & 0.058 & 0.177 \\
\hline 0.044 & 0.006 & 0.036 & 0.011 & 0.072 \\
\hline 0.039 & 0.005 & 0.076 & -0.017 & 0.011 \\
\hline 0.043 & 0.005 & -0.041 & 0.063 & 0.170 \\
\hline 0.049 & 0.003 & -0.069 & 0.082 & 0.205 \\
\hline
\end{tabular}

L/D

4.322

1.647

0.283

3.948

4.173

Elevon Wing, 3.5 degree AOA, $15.942 \mathrm{~m} / \mathrm{s}$ T038ET042E

\section{Control Position}

7
3.5
0
-3.5
-7

\begin{tabular}{|c|c|c|c|c|}
\multicolumn{1}{c}{ CD } & CmY & CmP & CmR & CL \\
\hline 0.030 & 0.008 & 0.013 & 0.025 & 0.080 \\
\hline 0.030 & 0.009 & 0.048 & 0.001 & 0.028 \\
\hline 0.030 & 0.008 & 0.080 & -0.021 & -0.011 \\
\hline 0.034 & 0.007 & -0.028 & 0.055 & 0.140 \\
\hline 0.038 & 0.005 & -0.058 & 0.075 & 0.175 \\
\hline
\end{tabular}

L/D

2.718

0.963

$-0.371$

4.064

4.590

Elevon Wing, 3.5 AOA,

$21.256 \mathrm{~m} / \mathrm{s}$ T043E-T047E

Control Position

7

3.5

0

$-3.5$

$-7$

\begin{tabular}{|c|c|c|c|c|}
\hline CD & CmY & CmP & CmR & CL \\
\hline 0.028 & 0.009 & 0.019 & 0.021 & 0.062 \\
\hline 0.025 & 0.010 & 0.051 & -0.001 & 0.021 \\
\hline 0.024 & 0.009 & 0.067 & -0.010 & 0.011 \\
\hline 0.029 & 0.008 & -0.025 & 0.054 & 0.131 \\
\hline 0.029 & 0.006 & -0.054 & 0.074 & 0.168 \\
\hline
\end{tabular}

L/D

2.228

0.847

0.446

4.455

5.771

Elevon Wing, 7 degree

AOA, $10.628 \mathrm{~m} / \mathrm{s}$ T048ET052E

\section{Control Position}

7
3.5
0
-3.5
-7

CD

\begin{tabular}{|l|l|l|l|l|}
\hline 0.087 & -0.002 & -0.112 & 0.132 & 0.421 \\
\hline 0.086 & -0.002 & -0.079 & 0.109 & 0.370 \\
\hline 0.096 & -0.002 & -0.037 & 0.080 & 0.312 \\
\hline 0.094 & -0.003 & -0.140 & 0.151 & 0.450 \\
\hline 0.097 & -0.005 & -0.168 & 0.171 & 0.491 \\
\hline
\end{tabular}

L/D

4.826

4.291

3.256

4.806

5.062

Elevon Wing, 7 degree AOA, 15.942 m/s T053ET057E 
Control Position

7

3.5

0

$-3.5$

$-7$

\begin{tabular}{|c|c|c|c|c|}
\multicolumn{1}{c}{ CD } & \multicolumn{1}{c}{ CmY } & \multicolumn{1}{c}{ CmP } & CmR & CL \\
\hline 0.072 & -0.001 & -0.115 & 0.135 & 0.414 \\
\hline 0.073 & 0.001 & -0.076 & 0.109 & 0.364 \\
\hline 0.072 & 0.000 & -0.033 & 0.075 & 0.290 \\
\hline 0.071 & -0.001 & -0.139 & 0.151 & 0.436 \\
\hline 0.074 & -0.004 & -0.165 & 0.170 & 0.475 \\
\hline
\end{tabular}

L/D

6.44707

6.119479

4.040

6.119

6.447

Elevon Wing, 7 degree

AOA, $21.256 \mathrm{~m} / \mathrm{s}$ T043E-

T062E

Control Position

7

3.5

0

$-3.5$

$-7$

\begin{tabular}{|c|c|c|c|c|}
\hline CD & \multicolumn{1}{c}{ CmY } & \multicolumn{1}{c}{ CmP } & CmR & CL \\
\hline 0.055 & 0.001 & -0.099 & 0.122 & 0.376 \\
\hline 0.055 & 0.002 & -0.061 & 0.096 & 0.326 \\
\hline 0.057 & 0.002 & -0.033 & 0.077 & 0.289 \\
\hline 0.053 & 0.000 & -0.130 & 0.143 & 0.410 \\
\hline 0.053 & -0.002 & -0.158 & 0.162 & 0.444 \\
\hline
\end{tabular}

L/D elevon

8.33

7.79

5.09

7.79

8.33

Elevon Wing, 7 degree

AOA, $28.026 \mathrm{~m} / \mathrm{s}$ T135E-

T040E

Control Position

7

3.5

0

$-3.5$

$-7$

\begin{tabular}{|c|c|c|c|c|}
\multicolumn{1}{c}{ CD } & \multicolumn{1}{c}{ CmY } & CmP & CmR & CL \\
\hline 0.034 & -0.002 & -0.089 & 0.121 & 0.376 \\
\hline 0.038 & -0.001 & -0.056 & 0.098 & 0.329 \\
\hline 0.040 & -0.001 & -0.031 & 0.081 & 0.302 \\
\hline 0.031 & -0.004 & -0.121 & 0.143 & 0.406 \\
\hline 0.030 & -0.006 & -0.149 & 0.156 & 0.437 \\
\hline
\end{tabular}

L/D

10.93

8.71

7.48

13.27

14.38 
Table 18 - Aerodynamic Coefficient Data for Morphing Windtunnel Model in WVU Wind Tunnel 2006-2007

Morphing Wing, 0 degree

AOA, $10 \mathrm{~m} / \mathrm{s}$ T063M-T067M

Control Position

-7
-3.5
0
3.5
7

\begin{tabular}{|c|c|c|c|c|}
\multicolumn{1}{c}{ CD } & CmY & CmP & CmR & CL \\
\hline 0.034 & 0.000 & 0.114 & -0.073 & -0.138 \\
\hline 0.028 & -0.006 & 0.155 & -0.109 & -0.191 \\
\hline 0.040 & -0.012 & 0.181 & -0.132 & -0.239 \\
\hline 0.018 & 0.001 & 0.057 & -0.032 & -0.048 \\
\hline 0.022 & 0.000 & 0.009 & 0.007 & 0.034 \\
\hline
\end{tabular}

L/D

$-4.036$

$-6.731$

$-5.958$

$-2.625$

1.517

Morphing Wing, 0 degree AOA, 15 m/s T068M-T072M

Control Position

-7
-3.5
0
3.5
7

\begin{tabular}{|c|c|c|c|c|}
\multicolumn{1}{c}{ CD } & \multicolumn{1}{c}{ CmY } & CmP & CmR & CL \\
\hline 0.015 & 0.001 & 0.086 & -0.053 & -0.086 \\
\hline 0.016 & -0.005 & 0.139 & -0.097 & -0.169 \\
\hline 0.022 & -0.009 & 0.159 & -0.110 & -0.195 \\
\hline 0.020 & 0.002 & 0.067 & -0.042 & -0.070 \\
\hline 0.016 & 0.002 & 0.010 & 0.009 & 0.027 \\
\hline
\end{tabular}

L/D

$-5.726$

$-10.638$

$-8.921$

$-3.431$

1.701

Morphing Wing, 0 degree AOA, $20 \mathrm{~m} / \mathrm{s}$ T073M-T077M

Control Position

$$
\begin{gathered}
-7 \\
-3.5 \\
0 \\
3.5 \\
7
\end{gathered}
$$

\begin{tabular}{|c|c|c|c|c|}
\multicolumn{1}{c}{ CD } & CmY & CmP & CmR & CL \\
\hline 0.017 & 0.002 & 0.071 & -0.040 & -0.064 \\
\hline 0.017 & -0.002 & 0.105 & -0.068 & -0.119 \\
\hline 0.015 & -0.007 & 0.138 & -0.091 & -0.154 \\
\hline 0.017 & 0.003 & 0.050 & -0.025 & -0.039 \\
\hline 0.017 & 0.003 & 0.001 & 0.018 & 0.050 \\
\hline
\end{tabular}

L/D

$-3.701$

$-7.032$

$-10.159$

$-2.279$

2.872

Morphing Wing,3.5 degree AOA, $10 \mathrm{~m} / \mathrm{s}$ T096M-T100M

Control Position

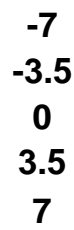

\begin{tabular}{|c|c|c|c|c|}
\multicolumn{1}{c}{ CD } & CmY & CmP & CmR & CL \\
\hline 0.077 & 0.001 & -0.079 & 0.103 & 0.338 \\
\hline 0.083 & 0.000 & -0.041 & 0.075 & 0.287 \\
\hline 0.083 & -0.002 & 0.020 & 0.026 & 0.184 \\
\hline 0.083 & 0.001 & -0.115 & 0.131 & 0.394 \\
\hline 0.087 & -0.003 & -0.170 & 0.173 & 0.465 \\
\hline
\end{tabular}


Morphing Wing,3.5 degree

AOA, 15 m/s T101M-T105M

Control Position

-7
-3.5
0
3.5
7

Morphing Wing, 3.5 degree AOA, $20 \mathrm{~m} / \mathrm{s}$ T106M-T110M

Control Position

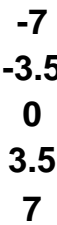

$-7$

$-3.5$

3.5

7

\begin{tabular}{|c|c|c|c|c|}
\multicolumn{1}{c}{ CD } & CmY & CmP & CmR & CL \\
\hline 0.053 & 0.002 & -0.073 & 0.096 & 0.302 \\
\hline 0.051 & 0.002 & -0.021 & 0.056 & 0.228 \\
\hline 0.050 & -0.001 & 0.010 & 0.033 & 0.184 \\
\hline 0.049 & 0.003 & -0.098 & 0.116 & 0.342 \\
\hline 0.052 & -0.001 & -0.149 & 0.157 & 0.422 \\
\hline
\end{tabular}

L/D

5.736

4.512

3.657

7.048

8.138

\begin{tabular}{|c|c|c|c|c|}
\multicolumn{1}{c}{ CD } & CmY & CmP & CmR & CL \\
\hline 0.038 & 0.004 & -0.064 & 0.090 & 0.287 \\
\hline 0.038 & 0.003 & -0.028 & 0.063 & 0.238 \\
\hline 0.039 & 0.001 & 0.002 & 0.041 & 0.199 \\
\hline 0.036 & 0.004 & -0.080 & 0.103 & 0.314 \\
\hline 0.034 & 0.001 & -0.128 & 0.140 & 0.376 \\
\hline
\end{tabular}

L/D

7.578

6.322

5.043

8.745

10.979

Morphing Wing, 7 degree AOA, $10 \mathrm{~m} / \mathrm{s}$ T111M-T115M Control Position

-7
-3.5
0
3.5
7

\begin{tabular}{|c|c|c|c|c|}
\multicolumn{1}{c}{ CD } & \multicolumn{1}{c}{ CmY } & CmP & CmR & CL \\
\hline 0.072 & -0.002 & -0.126 & 0.159 & 0.470 \\
\hline 0.073 & -0.002 & -0.089 & 0.129 & 0.417 \\
\hline 0.075 & -0.004 & -0.052 & 0.099 & 0.363 \\
\hline 0.073 & -0.003 & -0.146 & 0.173 & 0.490 \\
\hline 0.085 & -0.008 & -0.203 & 0.219 & 0.575 \\
\hline
\end{tabular}

L/D

6.555 5.685 4.814 6.743 6.750

Morphing Wing, 7 degree AOA, $15 \mathrm{~m} / \mathrm{s}$ T116M-T120M Control Position

$$
\begin{gathered}
-7 \\
-3.5 \\
0 \\
3.5 \\
7
\end{gathered}
$$

\begin{tabular}{|c|c|c|c|c|}
\multicolumn{1}{c}{ CD } & CmY & CmP & CmR & CL \\
\hline 0.055 & -0.001 & -0.125 & 0.157 & 0.460 \\
\hline 0.056 & -0.001 & -0.085 & 0.125 & 0.397 \\
\hline 0.057 & -0.002 & -0.047 & 0.096 & 0.354 \\
\hline 0.053 & -0.001 & -0.141 & 0.170 & 0.485 \\
\hline 0.055 & -0.006 & -0.201 & 0.217 & 0.567 \\
\hline
\end{tabular}

L/D

Morphing Wing, 7 degree AOA, $21 \mathrm{~m} / \mathrm{s}$ T121M-T125M

Control Position

$-7$
CD 0.036
CmY 0.001
CmP $-0.106$
CmR

0.138

CL 0.418
L/D 11.49 


\begin{tabular}{|c|c|c|c|c|c|c|}
\hline-3.5 & 0.039 & 0.001 & -0.082 & 0.120 & 0.386 & 9.97 \\
\hline 0 & 0.041 & 0.000 & -0.055 & 0.101 & 0.351 & 8.60 \\
\hline 3.5 & 0.036 & 0.000 & -0.126 & 0.153 & 0.439 & 12.36 \\
\hline 7 & 0.033 & -0.004 & -0.165 & 0.182 & 0.497 & 14.94 \\
\hline
\end{tabular}

Table 19 - Aerodynamic Coefficient Data for NACA 0015 Test Windtunnel Model in WVU Wind Tunnel 2007

\begin{tabular}{c|cc|c|c|c|}
$\begin{array}{c}\text { Re }=183,000 \\
\text { AOA }\end{array}$ & CD & CmY & CmP & CmR & CL \\
$\mathbf{0 . 0 0}$ & 0.011 & -0.014 & 0.009 & 0.005 & 0.017 \\
$\mathbf{2 . 0 0}$ & 0.023 & -0.025 & 0.077 & 0.129 & 0.223 \\
$\mathbf{5 . 0 0}$ & 0.040 & -0.029 & 0.080 & 0.214 & 0.490 \\
$\mathbf{1 0 . 0 0}$ & 0.060 & -0.040 & 0.096 & 0.325 & 0.762 \\
$\mathbf{1 2 . 0 0}$ & 0.070 & -0.048 & 0.114 & 0.374 & 0.880 \\
\hline
\end{tabular}




\section{Curriculum Vitae}

\section{Richard W. Guiler}

Mr. Guiler is an aerospace engineer with management, research, aerodynamics, propulsion, and composites experience. After 7 years in the environmental engineering field received a Masters of Science degree in aerospace engineering from West Virginia University in 1999. His masters research was for the U.S. Department of Energy on the operation and emissions of gas turbines operating on both aviation kerosene and methanol gas turbine project.

In 2000, Mr. Guiler attended the Deutsch Institute in Berlin Germany to learn the German Language. During this time Mr. Guiler developed a strong interest in the history and design of tailless aircraft. He had the opportunity to visit many of the historical sites associated with the development of tailless aircraft theory in Germany.

In 2001 he became the director of Engineering for Aurora Flight Science of West Virginia, an aerospace firm specializing in defense UAVs. He led the development and manufacturing efforts on many new UAVs including the beginning of the Northrop Grumman Global Hawk fuselage and wing construction. He also led the research effort to develop new honeycomb core sandwich structures for the F-14 to extend their lifespan as part of a U.S. Navy SBIR which has now gone on to a successful Phase III research project.

In September of 2002 he was presented the opportunity to begin earning a $\mathrm{PhD}$ in Aerospace at West Virginia University. While earning a $\mathrm{PhD} \mathrm{Mr}$. Guiler continued to work as a special projects engineer for Aurora Flight Science, teach an undergraduate UAV design course and conducted morphing tailless aircraft research funded by NASA Dryden Flight Research Center. .

In August 2007 Mr. Guiler earned a $\mathrm{PhD}$ in Aerospace Engineering from West Virginia University and accepted a Program Manager position for the Bell Eagle Eye UAV with Aurora Flight Sciences. 